\title{
Comparison of Airborne and Surface Particulate Size Distributions in Specific Hanford Nuclear Facilities
}

Prepared for the U.S. Department of Energy Office of Environmental Restoration and Waste Management

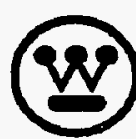

Hanford Operations and Engineering Contractor for the

U.S. Department of Energy under Contract DE-ACO6-87RL10930

Copyright License By acceptence of this article, the publisher and/or recipient acknowledges the

U.S. Government's right to retain a nonexclusive, royalty-free license in and to any copyright covering this paper.

Thesis This document was prepared in partial fulfillment of the requirements for a Mesters of Science degree in Rediological Sciences from the University of Washington, Seattio, Weshington. 
LEGAL DISCLAIMER.

This report was prepared as an account of work sponsored by an agency of the United States Government. Neither the United States Government nor any agency thereof, nor any of their employees, nor any of their contractors, subcontractors or their employees, makes any warranty, express or implied, or assumes any legal liability or responsibility for the accuracy, completeness, or any third party's use or the results of such use of any information, apparatus, product, or process disclosed, or represents that its use would not infringe privately owned rights. Reference herein to any specific commercial product, process, or service by trade name, trademark, manufacturer, or otherwise, does not necessarily constitute or imply its endorsement, recommendation, or favoring by the United States Government or any agency thereof or its contractors or subcontractors. The views and opinions of authors expressed herein do not necessarily state or reflect those of the United States Government or any agency thereof.

This report has been reproduced from the best available copy. Available in peper copy and microfiche.

Available to the U.S. Department of Energy

and its contractors from

Office of Scientific and Technical Information

P.O. Box 62

Oak Ridge, TN 37831

(615) $576-8401$

Available to the public from the U.S. Department of Commerce National Technical Information Service

5285 Port Royal Road

Springfield, VA 22161

(703) $487-4650$

Printed in the United States of America

DISCLM-1.CHP (1-91) 


\section{Comparison of Airborne and Surface Particulate Size Distributions in Specific Hanford Nuclear Facilities}

D. B. Ottley

Date Published

May 1995

Prepared for the U.S. Department of Energy Office of Environmental Restoration and Waste Management 


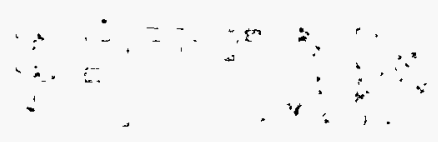




\section{DISCLAIMER}

Portions of this document may be illegible in electronic image products. Images are produced from the best available original document. 


\section{RELEASE AUTHORIZATION}

\section{Document Number: WHC-MR-0496}

Document Title: $\quad$ Comparison of Airborne and Surface Particulate Size

Distributions in Specific Hanford Nuclear Facilities

Release Date: $\quad 5 / 3 / 95$

This document was reviewed following the procedures described in WHC-CM-3-4 and is:

APPROVED FOR PUBLIC RELEASE

WHC Information Release Administration Specialist:

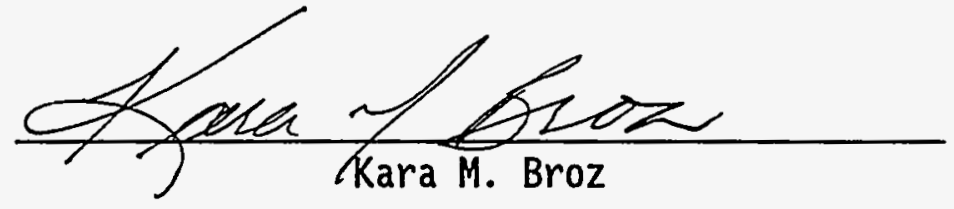

May 3, 1995 

University of Washington

\title{
Abstract
}

\section{COMPARISON OF AIRBORNE AND SURFACE PARTICULATE SIZE DISTRIBUTIONS IN SPECIFIC HANFORD NUCLEAR FACILITIES}

\author{
by David B. Ottley \\ Chairperson of the Supervisory Committee: Professor Michael S. Morgan \\ Department of Environmental Health
}

Settled dust from nuclear operations may be contaminated with radionuclides and become resuspended and subsequently breathed. This is the predominate radionuclide inhalation hazard scenario in nuclear facilities that have been deactivated and no longer have liquid in their process systems that may become directly airborne in accident situations. Comparisons were made between the indoor ambient airborne particulate size distribution and that of resuspended dust that could become contaminated and subsequentiy airborne during decommissioning operations at selected nuclear facilities on the Hanford Site. The results indicate that only $5 \%$ of the particles, by count, above the breathing zone are greater than ten (10) $\mu \mathrm{m}$ in size and that the particulates that could be resuspended into the breathing zone had a mean aerodynamic equivalent diameter of four (4) $\mu \mathrm{m}$ or less. 

HHC-MR-0496

\section{CONTENTS}

Page

List of figures . . . . . . . . . . . . . . . . . vi

List of Tables . . . . . . . . . . . . . . . . . vii

List of Acronyms . . . . . . . . . . . . . . . . viii

Definitions . . . . . . . . . . . . . . . . . ix

Introduction ....................... . . 1

Background . .. . . . . . . . . . . . . . . 2

Chapter I: Methods .................... 6

Sampling Protocol . . . . . . . . . . . . . . 6

Equipment ..................... . 6

Sampling Locations . . . . . . . . . . . . . . 10

Documentation ................... . . 11

Precision and Accuracy ................. . . 11

Cascade Impactors . . . . . . . . . . . . . . 13

Chapter II: Results . . . . . . . . . . . . . . . 16

Ambient Air .................. 16

Resuspension ..................... 19

Analysis . . . . . . . . . . . . . . . . 29

Chapter III: Conclusions . . . . . . . . . . . . . 35

References ....................... 36

Appendix A: AED Conversion from OD . . . . . . . . . . 41

Appendix B: Instruction, Operation of Laser Particle Counters . . 43

Appendix C: Aerosol Particle Measurement Record . . . . . . . 46 


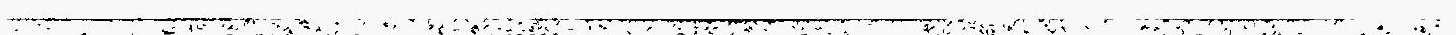




\section{CONTENTS (Continued)}

Page

Appendix D: Instruction, Assembly/Operation of Cascade Impactor . 47 Appendix E: Cascade Impactor Cut-Points Versus Flow Rate . . . 49 Appendix F: Cascade Impactor Substrates . . . . . . . . 50 Appendix G: Laser Particle Counter ........... . 51 Appendix H: Aerosol Particle Measurement Record Data Sheets . . . 52 


\section{LIST OF FIGURES}

Number

Page

1. Ambient Air Vs. Sample Height, Building 3706, Room $102 \ldots 15$

2. Mean Optical Diameter Vs. Time Following Resuspension, 3706A . 22

3. Resuspension and Ambient Air Vs. Sample Height, B7dg. 3706A . 26

4. Resuspension and Ambient Air Vs. Sample Height, 3706, Rm. 10227

5. Resuspension and Ambient Air Vs. Sample Height, PUREX . . . 28 



\section{LIST OF TABLES}

Number

Page

1. Bin Range, Diameter and Diameter Width ........ 9

2. Comparison Between the Two Laser Particle Counters .... 13

3. Mean Optical Diameter of Ambient Air in Building 3706A . . 17

4. Mean Optical Diameter of Ambient Air in B7dg. 3706, Rm. 120 . 18

5. Mean Optical Diameter of Ambient Air in PUREX ...... 19

6. Peak Mean Diameter $(\mu \mathrm{m})$, Versus Sampling Height . . . . 24

7. Percent of Large Particles in Breathing Zone, Bldg. 3706A . . 30

8. Percent of Large Particles in Breathing Zone, 3706, Rm. 102 . 31

9. Percent of Large Particles in Breathing Zone, PUREX . . . . 32

10. Mean Percent of Large Particles in Breathing Zone . . . . . 32

11. Mean Optical Diameter as a Function of Height and Correlation 34 


\section{LIST OF ACRONYMS}

AED

AMAD

CAM

D

LPC

OD

P\&O

RCA

WHC
Aerodynamic Equivalent Diameter

Activity Median Aerodynamic Diameter

Continuous Air Monitor

Density

Laser Particle Counter

Optical Diameter

Pipe and Operating

Radiological Controlled Area

Westinghouse Hanford Company 


\section{DEFINITIONS}

AED Aerodynamic Equivalent Diameter, the diameter of unit density sphere having the same settling velocity

AMAD Activity Median Aerodynamic Diameter, the diameter of uniform unit density sphere having the same settling velocity as the particle carrying the median amount of radioactivity in the particle distribution

OD

Optical Diameter, the diameter of instrument calibration particles (polystyrene latex spheres) that scatters light into the receiving optics with the same amplitude as the particle actually in the sensing zone 


\section{ACKNOWLEDGMENTS}

The author wishes to express sincere appreciation to Professors Michael Morgan, Ronald Kathren, Maurice Robkin, and Gene Schreckhise, al so to John G7issmeyer and William Eadie for their assistance in the preparation of this manuscript and to my wife, Charlene for assistance in typing. 
WHC-MR-0496

\section{INTRODUCTION}

It is important to know the size distribution of particulates in worker's breathing air because of the relationship between particle size and respirability. Previous studies to ascertain the airborne particle size distribution at nuclear facilities have centered on the particle size distribution in liquid process systems (Andersen and Nelson 1967; Perrin 1987). The assumption has been that as a system break occurs, the material within the system becomes airborne. This would be the case in a catastrophic accident but not Tikely in contamination incidents resulting from routine operation.

In routine operations, process stream particle size distributions are less important than the indoor dust particle size. Breaches in process streams contaminate the settled dust on floors and other surfaces, which in turn becomes resuspended. The resuspension of radioactive particulates is the predominant inhalation and worker internal exposure scenario for a facility that has been deactivated and no longer has Tiquid in its process system. The hypothesis of this study is that the size distribution of resuspended dust contains too few particles of aerodynamic diameters greater than or equal to $10 \mu \mathrm{m}$ to use $10 \mu \mathrm{m}$ as the default particle size for air sampling transport line loss determinations. If supported, a more realistic maximum aerodynamic diameter based on typical data can be recommended for use as a default. when the actual particle size distribution is not known. Selected 
Hanford Site nuclear facilities were used to determine and compare ambient and resuspended indoor dust particle size distributions.

\section{Background}

Ambient air samples are normally taken in strategic locations to detect and evaluate airborne radioactive material in most nuclear facilities. It is desirable that these samples be representative of worker's breathing air. Continuous air monitors (CAMs), which are often used for this purpose, may be remotely situated from the actual sampling location to minimize occupational radiation exposure to workers who must maintain them. For these installations, an air sample is drawn through a transport line which may take a circuitous route to the CAM filter. As the air travels through the transport line, particles are deposited on the walls of the transport line tubing in a size discriminative manner (ANSI 1969; USNRC 1991). Inertial effects on larger particles will cause them to impact and accumulate on bends in the transport line because they are too large to traverse the curve (Fan et al. 1992). If the concentration of radionuclides in the larger (or smal1) particles significantiy differs from the concentration in the particles in the respirable range, the radioactive material collected on the filter may not be representative of the worker's breathing air or of the dose delivered to the respiratory system. Furthermore, nonrepresentative sampling may lead to inadequate determination of respiratory protection needs for workers and the possibility of errors in determining doses from inhaled particles (McFarland et al. 1991). 
At Westinghouse Hanford Company (WHC), the particle size assigned to facilities with airborne radioactive systems containing mixed fission products (i.e., beta/gamma emitters) has been seven (7) $\mu$ m aerodynamic . equivalent diameter (AED) (Rathbun 1992). The particle size assigned to systems containing plutonium compounds (i.e., alpha emitters) has been four (4) $\mu \mathrm{m}$, AED (Ruben 1992b).

Computer programs such as DEPOSITION (Anand et a1. 1991, 1993) can be used to quantify the air sampling transport line loss (Glissmeyer 1991). Without particle size distribution knowledge, conservative assumptions must be made in the computer analysis; typically a $10 \mu \mathrm{m}$ diameter size is chosen as the worst case. This is based on the following recommendation by the U.S. Nuclear Regulatory Commission: "The penetration of particles with an aerodynamic equivalent diameter of 10 micrometers should be at least 50 percent," (USNRC 1992). The penetration of a particle is its ability to traverse the transport line and deposit on the filter, and is the opposite of deposition, when the particle deposits on the transport line wall.

A particle size of ten (10) $\mu \mathrm{m}$ was selected because $10 \mu \mathrm{m}$ AED is considered the diameter at which not more than $1 \%$ of the ambient particles enter the pulmonary zone of the lungs (ACGIH 1985). However, if there are few or no particles with $10 \mu \mathrm{m}$ AED or larger diameter size, then it is not appropriate to evaluate a transport line at this size. A suitably conservative default value would be the upper size of the particle range that covered $95 \%$ of the particles of interest. This paper will show that evaluating airborne radioactive material sampling 
WHC-MR-0496

transport lines by using the default size of $10 \mu \mathrm{m}$ for particles is too conservative - i.e. overstates the radionuclide concentration - allowing a greater safety margin than is warranted for most applications. Currently, if a sample line does not meet acceptance criteria, the line must either be replaced or its use discontinued. Overly conservative assumptions may result in rejection of an excessive number of transport lines with them not being accepted for use and consequent unwarranted upgrade costs.

A sphere increases in volume and subsequent mass as its diameter cubed, whereas surface area increases as the diameter squared. If the entire particle is considered to be made up of radioactive material, then a 10-fold increase in particle diameter results in a 1,000-fold increase in radioactivity. This would be the case in the resuspension of a radioactive liquid. If the floor dust becomes coated with the radioactive 7 iquid and then is resuspended, the 10-fold increase in diameter results in only a 100-fold increase of surface area and subsequent radioactivity.

Another use of particle size distribution knowledge is for determination of the dose to the respiratory tract from inhaled radioactive material. Currently at the Hanford Site, dose assessment is based on the Reference Man put forth by the International Commission of Radiological Protection, ICRP (ICRP 75) and an assumed particle size of one (1) $\mu \mathrm{m}$ AMAD (Sula et al. 1989). The Computer-based INternal Dosimetry System (CINDY) computer model (Strenge et al. 1992) in use at some nuclear facilities, allows the input of event-specific particle 
size information making it possible to individualize the incident assessment if the size distribution of the inhaled particle is known. This yields a more accurate dose assessment from the inhalation of radioactive particles. 


\section{CHAPTER I: METHODS}

Particle size distributions in ambient air were determined and compared to those from resuspended settled dust collected at various sampling heights as described below.

\section{Sampling Protocol}

A dust-free, non-absorbing cloth, (air sampling filter paper) was used to gently remove a sample of settled dust, with care taken not to infuse the dust into the cloth. The dust was then resuspended at the orifice of the sampling probe of a laser particle counter (LPC) by delicately agitating the cloth. This sampling technique was designed to ensure that the particle size distribution was not affected by this LPC sampling protocol. However, the results of this effort were not reproducible. This is consistent with other experimenters (e.g., Caplan 1993). Therefore, a jig was assembled to sample airborne particies after the settled dust was resuspended with a small horsehair brush. As noted by Sehmel (1979), vigorous sweeping has a very high mechanical resuspension factor. This method ensured that a particle distribution shift did not occur due to sampling protocol and that sampling would be reproducible as it has been for others (e.g., Fish 1964).

\section{Equipment}

Laser particle counters, Mode $1200 \mathrm{~L}$, manufactured by Met One, 
WHC-MR-0496

Inc. (Met One 1989) as pictured in Appendix G, were used to determine particle size distributions. Laser particle counters (LPC), measure optical diameter (OD), or physical size (Gouesbet and Grehan 1988), as opposed to aerodynamic equivalent diameter (AED), which takes into consideration the particle density. LPCs have the ability to determine particle size distributions of unknown aerosols including dust (Benjamin et al. 1974; Chen and Cheng 1984; Killinger and Zerul1 1984). No correction was made for the difference in refractive index of dust particles measured in this study versus calibration particles. Another measure of particle size is activity median aerodynamic diameter (AMAD) which additionally considers the radioactivity distribution within the particle size range. Radioactivity distributions or AMADs were not calculated for this study. Optical diameter measurements were used in this study for comparison purposes to determine size variabilities. These optical diameter results can be compared with the AED values. in the literature by a simple conversion. The AED can be approximated by multiplying the $O D$ values by 1.5 (see Appendix $A$ for derivation), which is consistent with conversion methods reported by Marple and Rubow (1976).

The Met One laser particle counter is a microprocessorcontrolled, laser-based counter that measures six particle-size ranges simultaneously ranging from $0.3 \mu \mathrm{m}$ to greater than $10 \mu \mathrm{m}$ OD. It displays the number of particles in each range as a cumulative or

* Met One Inc., Grants Pass, Oregon 
differential count. The coincidence error is less than $10 \%$ at a concentration of 140 particles $/ \mathrm{cm}^{3}\left(4,000,000\right.$ particles $\left./ \mathrm{ft}^{3}\right)$. It is equipped with adjustable count alarms and automatic calibration verification.

The laser particle counter uses a solid-state laser diode and lenses to create an infrared light beam that senses the particles. The particles pass through the infrared beam, deflecting bursts of light onto a solid-state photo-diode that converts the light into an electrical pulse. The size of the pulse is proportional to the particle size.

The sampled air flows through a sample head at $28 \mathrm{l} / \mathrm{m}$ (1 cfm). A built-in printer provides particle-count printouts at timed intervals; time and date are automatically noted on the printout. The laser particle counters were operated according to the manufacturer's recommendations and the attached procedure (Appendix $B$ ).

The laser particle counter sorts the counted particles into six separate bins (Table 1). The mean bin diameter, $d$, represents the geometric mean particle size collected in that particular bin and is calculated as the square root of the product of the lower, $\left(d_{1}\right)$ and upper, $\left(d_{u}\right)$ size range cut-off points, $\left(d_{l} * d_{u}\right)^{1 / 2}$. Since the bin upper cut-off point is approximately twice the value of the lower cut-off point, the upper cut-off point for the last bin was chosen as 20, twice 10 so that a bin mean diameter could be calculated. There are few respirable particles larger than $20 \mu \mathrm{m} 0 \mathrm{D}$. Additionally, this makes the bin width comparable with the other bin widths. 
WHC-MR-0496

The count-weighted mean diameter is the summation of the product of each mean bin diameter, $d$ and particle count, divided by the total count, $\Sigma(d *$ count $) \div \Sigma$ count and has been calculated for each test performed.

The count-weighted size can be plotted against the bin diameter to graphically show particle size distribution; however the plot of ambient air size distribution is often bimodal and thus it is difficult to use in determining the mean particle size (Hinds 1992; Hickey et al. 1993). Therefore, graphing was not performed for this study.

Instead, the mean particle size was determined mathematically by establishing suitable algorithms as subroutines in the data entry program to automatically calculate the count-weighted mean diameter. The completed data sheets showing the calculated values are contained in Appendix H.

Table 1. Bin Range, Diameter and Diameter Width.

\begin{tabular}{ccc}
$\begin{array}{c}\text { Bin Range } \\
O D\end{array}$ & $\begin{array}{c}\text { Mean Bin } \\
\text { Diameter } \\
(\mu \mathrm{m})\end{array}$ & $\begin{array}{c}\text { Diameter } \\
\text { Width } \\
\Delta \operatorname{log~d} .\end{array}$ \\
\hline $0.30-0.49$ & 0.38 & 0.21 \\
\hline $0.50-0.99$ & 0.70 & 0.30 \\
\hline $1.00-1.99$ & 1.41 & 0.30 \\
\hline $2.00-4.99$ & 3.16 & 0.40 \\
\hline $5.00-9.99$ & 7.07 & 0.30 \\
\hline$>10.0$ & 14.14 & 0.30
\end{tabular}




\section{Sampling Locations}

Measurements of indoor particle size distributions were made at locations in three separate buildings on the Hanford Site: 1) 3706 Building, room 102, 2) 3706A Building, and 3) PUREX. The 3706 Building is a 50-year old wood-frame office structure which was once used as the plutonium separations process development laboratory. The attic of the building has been designated as a radiologically controlled area (RCA) because of potential plutonium contamination remaining in the old radiochemical hood ventilation duct work. Therefore, it will be demolished as an RCA-designated facility.

The 3706A Building served as a utilities building for water, steam, and electrical services to support the 3706 Building before it was converted to an office. The $3706 \mathrm{~A}$ Building is a concrete structure with openings under the doors which have permitted dust ingress and contributed to a large accumulation of dust in the building. This made it particularly useful for this study because in normally occupied buildings, dust accumulation is obviated by routine cleaning and therefore not readily available for resuspension studies.

The PUREX facility is also of concrete construction and is the newest plutonium separations facility, or canyon building at the Hanford Site having begun operation in January 1956. Separation functions were terminated in 1990 and the building is currently being prepared for decommissioning.

These three facilities (i.e., 3706A, 3706 and PUREX) are typical of the radiologically controlled facilities at the Hanford Site. 
WHC-MR-0496

Particulate size information will be useful because air sampling will be performed in these and similar facilities providing information to protect workers from potential airborne contaminants as demolition takes place.

\section{Documentation}

Data from the laser particle counters were entered onto the Particulate Measurement Record form (Appendix $C$ ) along with the sample location. The matching printout from the LPC was attached to the Particle Measurement Record form. Additional measurements of temperature, humidity, dew point and (if applicable) wind speed were performed with a TSI Veloci Calc Plus model 8360 Anemometer $^{*}$. These data were also recorded on the form.

\section{Precision and Accuracy}

The precision or reproducability of the two laser particle counters used in this study was quantitatively determined by obtaining multiple counts under the same conditions in each particle size range and comparing the mean of the data between the two LPCs. The accuracy was determined by qualitatively comparing the mean aerodynamic diameter to the mean aerodynamic diameter measured with a cascade impactor.

The two laser particle counters were denoted \#1 and \#2 for serial

*Veloci Calc P7us is a trademark of the TSI company, Minneapolis, Minnesota. 
WHC-MR-0496

numbers, 9002979A and 891601209 respectively. Both particle counters were set up according to the manufacturer's instructions and were operated according to the attached procedure (Appendix $B$ ).

Probes for the laser particle counters were positioned to sample ambient air at a height of one meter above the floor with the probe inlets facing horizontally the same direction, while spaced 0.5 meters away from each other. The length of the probe sampling lines for each LPC was minimized to an equal length of 0.5 meters thus equalizing deposition within. the lines for the two probes. Sampling flow rate was set at $281 / \mathrm{m}(1 \mathrm{cfm})$. The counters were set to take 10 consecutive one-minute samples in room 102 of the 3706 Building. The room was undisturbed during the sampling, except by the normal air flow caused by drafts and air conditioning.

Anemometer data recorded at the beginning of the sampling period along with the number of counts per size range were entered into a computer-based algorithm where the mean optical diameter was calculated. The difference, mean, and percent difference for the 10 consecutive samples was then calculated to determine a mean percent difference. The coefficient of variation of the means is $4.1 \%$ as is shown in Table 2 . By means of this comparison the two laser particle counters have been shown to yield precise and similar results. 
Table 2. Comparison Between the Two Laser Particle Counters.

\begin{tabular}{|c|c|c|c|c|c|}
\hline & \multicolumn{2}{|c|}{ Hean Diameter $(\mu / \mathrm{m})$} & \multirow{2}{*}{$\begin{array}{c}\text { Difference } \\
\qquad(\mu m)\end{array}$} & \multirow{2}{*}{$\begin{array}{l}\text { Mean } \\
(\mu m)\end{array}$} & \multirow[t]{2}{*}{$\%$ Difference } \\
\hline & Sampler \#1 & Sampler \#2 & & & \\
\hline & 0.64 & 0.65 & 0.01 & 0.65 & 1.5 \\
\hline & 0.61 & 0.62 & 0.01 & 0.62 & 1.6 \\
\hline & 0.63 & 0.63 & 0.00 & 0.63 & 0 \\
\hline & 0.60 & 0.61 & 0.01 & 0.61 & 1.6 \\
\hline & $0 . \dot{59}$ & 0.60 & 0.01 & 0.60 & 1.7 \\
\hline & 0.58 & 0.60 & 0.02 & 0.59 & 3.4 \\
\hline & 0.57 & 0.57 & 0.00 & 0.57 & 0 \\
\hline & 0.58 & 0.58 & 0.00 & 0.58 & 0 \\
\hline & 0.61 & 0.62 & 0.01 & 0.62 & 1.6 \\
\hline & 0.58 & 0.60 & 0.02 & 0.59 & 3.4 \\
\hline Mean & 0.60 & 0.61 & 0.01 & 0.61 & 1.5 \\
\hline Standa & Deviation & Means & & 0.025 & \\
\hline
\end{tabular}

\section{Cascade Impactors}

The accuracy of the two laser particle counters used in this study was determined by comparing there results with that of a cascade impactor. Cascade impactors are frequently used in determining particle size distributions. However, for this type of study, they were determined to not be appropriate as they require long sampling times and do not reflect momentary or real time changes in the environment (Yu et 
WHC-MR-0496

a1. 1993). Laser particle counters were used in this study because of their sensitivity to sudden shifts in particle size distributions. A qualitative comparison between the laser particle counters and a cascade impactor was performed to determine the laser particle samplers sizing ability.

An Andersen cascade impactor ${ }^{*}$, Series 210, was assembled and operated according to the procedure described in Appendix D (Andersen 1979) in order to sample ambient air. The impactor sample was collected in the 3706 Building, room 102 at a flow rate of 3 1pm for 24 hours. The cut-off points for the filter substrates were determined by the flow rate selected so the degree of filter loading could be used to qualitatively confirm the particle-sizing ability of the laser particle counters. Quantitative evaluation of results was not conducted because of the difficulty of accurately weighing the substrates. A copy of the "Cut-Points Versus Flow Rate" graph is attached in Appendix E. Photocopies of the filter substrates are included in Appendix F.

Visual analysis of the loading on the filter substrates shows heavy loading on the three filter substrates in the ranges from $0.076 \mu \mathrm{m}-0.86 \mu \mathrm{m}$ AED (i.e., less than $1.0 \mu \mathrm{m}$ AED). The ambient air particle size determined using the laser particle counter within the same room (Fig. 1), was approximately a $0.6 \mu \mathrm{m}$ OD. When this LPC

* Andersen Cascade Impactor, available from Andersen Samplers Inc., Atlanta, Georgia. 
outcome is multiplied by the 1.5 conversion factor (as derived in Appendix A) for comparative purposes, the resulting $1.0 \mu \mathrm{m} \mathrm{AED} \mathrm{is} \mathrm{the}$ same as the visual estimated maximum obtained using the cascade impactor. This demonstrates that the LPC and cascade impactor are comparable within the limitations of this study.

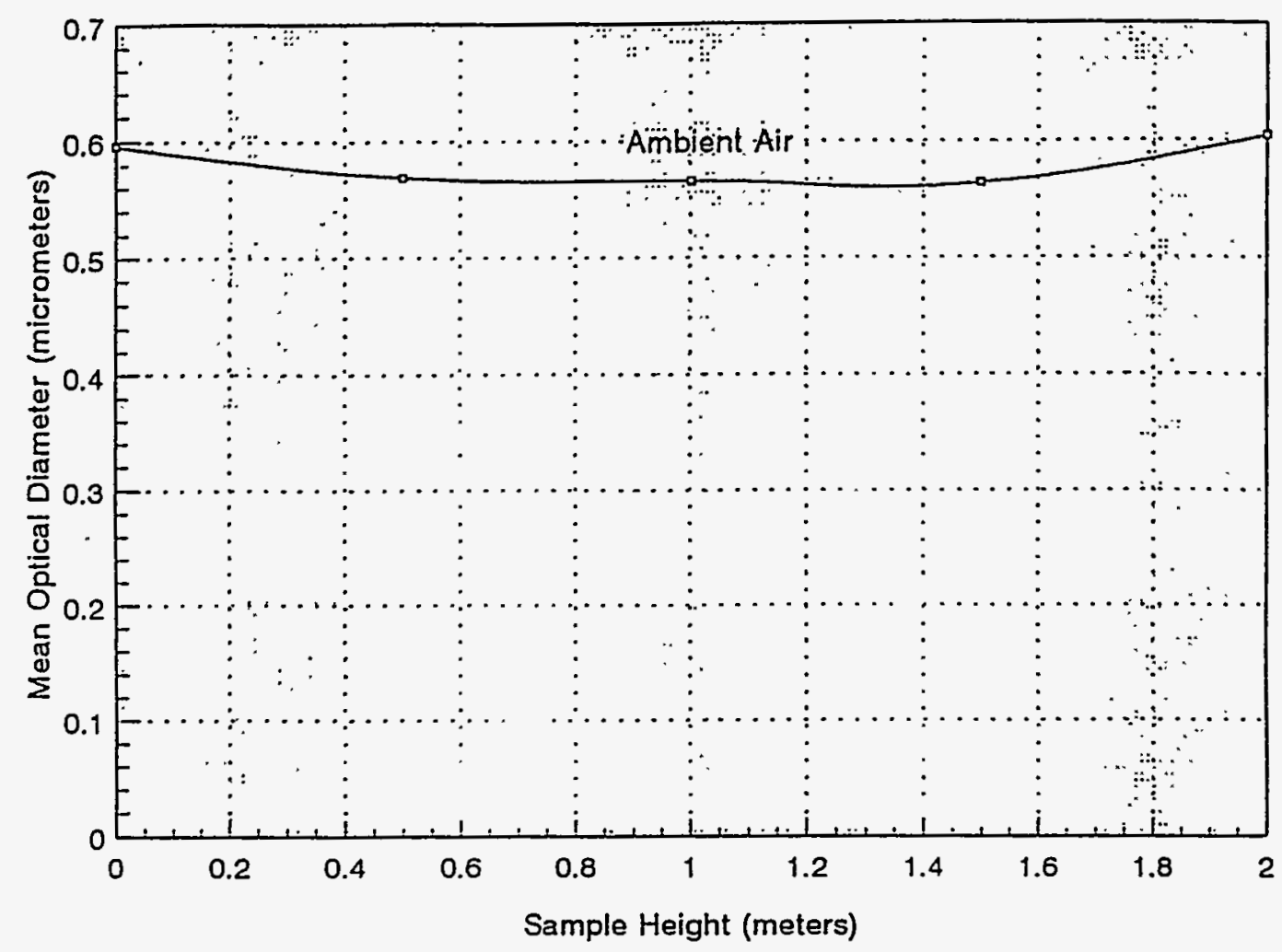

Fig. 1. Ambient Air Vs. Sample Height, Building 3706, Room 102.

In a previous study i.e., (Ruben 1992a), a cascade impactor sample was obtained from cell 9 of the B-Plant canyon. The B-Plant canyon is similar but older than the PUREX facility. Results demonstrated that the mean particle size was approximately $1.0 \mu \mathrm{m}$. 


\section{CHAPTER II: RESULTS}

The following experiments were performed to establish the ambient air particle size and determine the variability in particle size versus height due to the resuspension of settled dust within the studied facilities:

\section{Ambient Air}

The mean optical diameter of ambient air particles as a function of height above the floor was determined by collecting 10, one-minute samples at five different heights with a $0.5 \mathrm{~m}$ increments between 0.02 and $2 \mathrm{~m}(0.02,0.5,1.0,1.5$, and $2.0 \mathrm{~m})$ in the 3706 and $3706 \mathrm{~A}$ buildings. The lowest practical height obtainable was $2 \mathrm{~cm}(0.02 \mathrm{~m})$ because this was as close to the floor as the probe could be placed for measurement. The sampling procedure 1isted in Appendix B was used. The data were entered into an Aerosol Particle Record form to determine the mean optical diameter for each sample. Each group of 10 samples was averaged and the standard deviation was calculated as shown in Tables 3 and 4. The small standard deviation indicates that there is very little variation between each of the 10 samples from each sample height. 
Table 3. Mean Optical Diameter of Ambient Air in Building 3706A.

\begin{tabular}{lccc}
$\begin{array}{c}\text { Sempling } \\
\text { Height } \\
(\mathrm{m})\end{array}$ & $\begin{array}{c}\text { Mean OD } \\
\text { 10 Measurements at } \\
\text { each height }(\mu \mathrm{m})\end{array}$ & $\begin{array}{c}\text { Standard } \\
\text { Deviation } \\
(\mu \mathrm{m})\end{array}$ & $\begin{array}{c}\text { Coefficient of } \\
\text { Variation }\end{array}$ \\
\hline 0.02 & 0.596 & 0.005 & $1 \%$ \\
0.5 & 0.569 & 0.006 & $1 \%$ \\
1.0 & 0.566 & 0.007 & $1 \%$ \\
1.5 & 0.564 & 0.007 & $2 \%$ \\
2.0 & 0.604 & 0.014 & \\
\hline Mean & 0.58 & 0.008 & \\
\hline Standard & 0.018 & & \\
Deviation & & & \\
of Means & & & \\
Coefficient of & $3.1 \%$ & & \\
Variation of & & & \\
Means & & & \\
\hline
\end{tabular}

The mean optical diameter of particles in the ambient air as a. function of height was quite consistent as no statistically significant differences were observed (Tables 3 and 4 ). The coefficient of variation of the means for the $3706 \mathrm{~A}$ Building was $3.1 \%$, and for the 3706 Building it was $3.2 \%$. 
Table 4. Mean Optical Diameter of Ambient Air in Bldg. 3706, Rm. 102.

\begin{tabular}{ccc}
$\begin{array}{c}\text { Sampling } \\
\text { Height } \\
\mathrm{m}\end{array}$ & $\begin{array}{c}\text { Mean } 00 \\
10 \text { Measurements } \\
\text { at each height }(\mu \mathrm{m})\end{array}$ & $\begin{array}{c}\text { Standard Deviation } \\
(\mu \mathrm{m})\end{array}$ \\
\hline 0.02 & 0.646 & 0.013 \\
0.5 & 0.636 & 0.032 \\
1.0 & 0.621 & 0.026 \\
1.5 & 0.612 & 0.011 \\
2.0 & 0.596 & 0.015 \\
\hline Mean $\quad-$ & 0.622 & 0.02 \\
\hline Standard Deviation of Means & 0.02 & \\
\hline Coefficient of Variation of Means & $3.2 \%$ &
\end{tabular}

The PUREX facility has higher radiation exposure rates than the 3706 or $3706 \mathrm{~A}$ facilities. Therefore, to reduce personnel radiation dose and because enough data were available from the other less contaminated facilities, ambient air sampling was performed only once at each increment of height in the PUREX facility. Mean optical diameter values for the PUREX facility are shown in Table 5 and are also very close to the more accessible, 3706 and $3706 \mathrm{~A}$ facilities. The coefficient of variation for PUREX (i.e.,9.1\%) is higher than the other two facilities. 
Table 5. Mean Optical Diameter of Ambient Air in PUREX.

\begin{tabular}{cc} 
Sampl ing Height $\mathrm{m}$ & Mean $00(\mu \mathrm{m})$ \\
\hline 0.02 & 0.76 \\
0.5 & 0.64 \\
1.0 & 0.65 \\
1.5 & 0.63 \\
2.0 & 0.62 \\
\hline Mean & 0.66 \\
\hline Standard Deviation of Mean & 0.06 \\
\hline Coefficient of Variation of Mean & $9.1 \%$
\end{tabular}

The results from the three indoor air environments studied indicate that the mean optical diameter of ambient indoor air ranges approximately between $0.5 \mu \mathrm{m}$ and $0.8 \mu \mathrm{m}$ with a mean of $0.62 \mu \mathrm{m}$. There was a tendency for larger particles to be near the floor which is expected due to the effects of gravitational settling. Converting the mean $0.62 \mu \mathrm{m}$ optical diameter to an aerodynamic equivalent diameter results in an approximately $0.93 \mu \mathrm{m}$ or about $1.0 \mu \mathrm{m}$ AED size particle. This is consistent with the size determined while establishing the precision of the laser particle counters (Table 2) and with the expected indoor particle size such as used for calculating internal doses at the Hanford Site (Sula et a7. 1989).

\section{Resuspension}

The purpose of the next experiment was to determine the size distribution of the resuspended settled dust versus time. Dust in 
WHC-MR-0496

indoor environments is primarily tracked in by foot traffic and/or blown in by winds (Owen et a7. 1992). In routine decommissioning operations, the process of sweeping the floors is one of the major causes of resuspending dust. Frequently water, such as in mopping, is used to contain airborne radioactive material. For this experiment a brush was used to agitate and resuspend dust from the floors.

To measure the particle size distribution of the resuspended. dust, the laser particle counters were operated according to the attached procedure (Appendix B).. The probes of the particle counters were oriented downwards to avoid collecting falling dust (Alzona et al. 1979). The first probe was positioned at $25 \mathrm{~cm}(0.25 \mathrm{~m})$ above the floor. This is as close to the floor as practical, yet being directly above the agitating brush. The other probe was placed at $1.5 \mathrm{~m}$ above the floor.

The laser particle counters were setup to sample simultaneousiy, taking 20-second samples separated by 40 -second hold times. The hold time was needed to allow the laser particle counters to stabilize between counts because of the large number of particles being counted. The hold time also minimized the probability of a particle being deposited in the sample line during one test from being resuspended in a subsequent test because it gave time for the sample line to clear. The first sample was of ambient air. Midway through the hold time between the first and second counts, a brush was used on the floor to stir up dust simulating demolition activities. The brush was used for about 
15 seconds within a one-meter radius of the probes to resuspend the dust. After the resuspension period, sampling continued for another 13 minutes, ample time for the laser particle counters to indicate particle counts had returned to near ambient values.

The settled dust was resuspended into a "cloud" in which the larger particles settle out and the smaller particles rose and dissipated into the air. The rate at which this process happens may vary based on such factors as air drafts, humidity, ceiling height, initial velocity, and particle shape and size.

This study was particularly concerned with the peak particle size measured after the dust resuspension event. The plot of the largest mean optical particle diameter versus time is shown in Fig. 2 for a test performed in the 3706A Building. The large peak observed at one minute for the $0.25 \mathrm{~m}$ sampling height is typical of what happens near the floor when the dust is initially resuspended. No change was seen in the $1.5 \mathrm{~m}$ sample until approximately four minutes later and then, to a much less extent. 


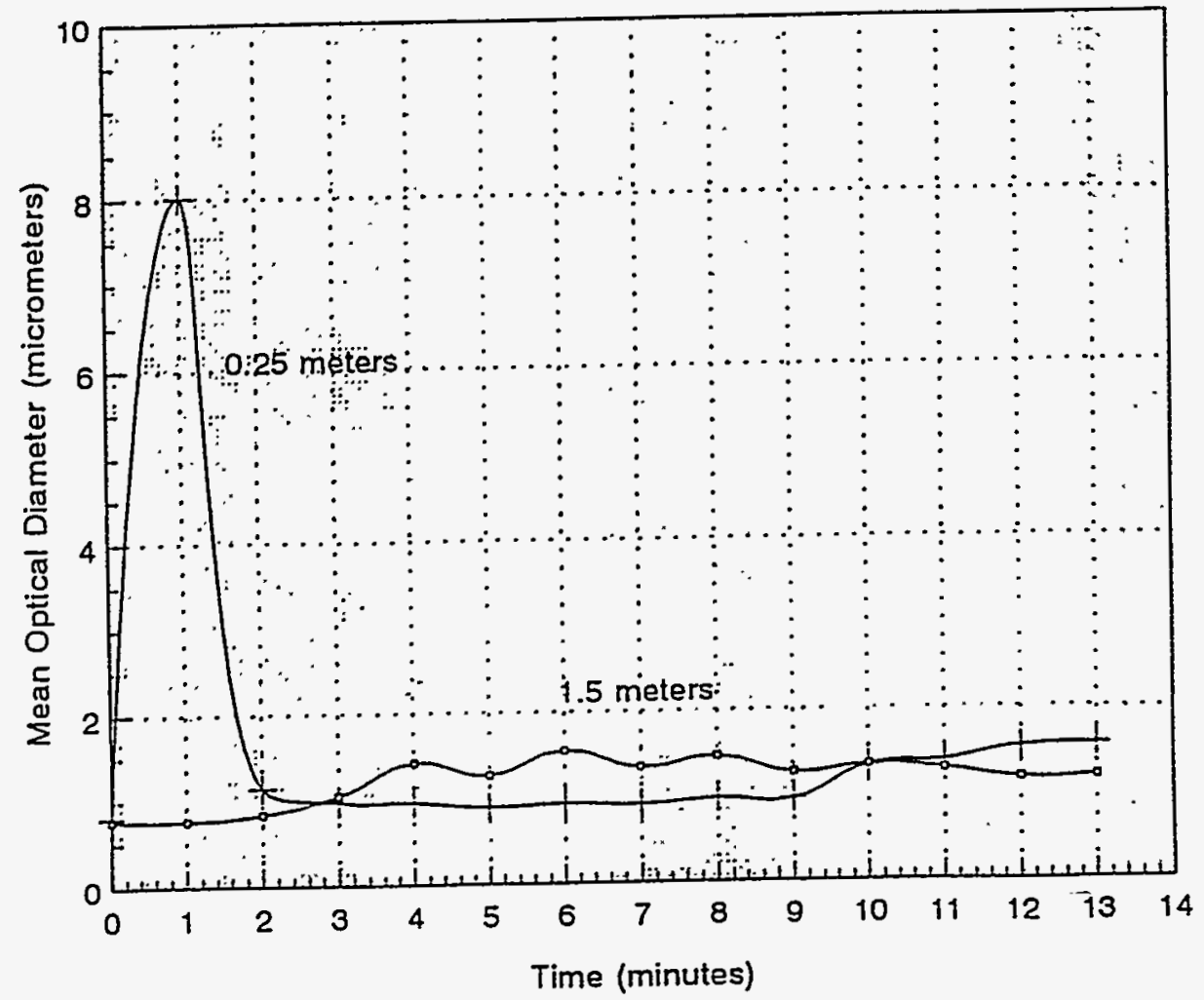

Fig. 2. Mean Optical Diameter Vs. Time Following Resuspension, 3706A

Twelve additional tests, such as the previous experiment, were performed in the 3706A Building. During each test, one probe sampled the resuspended dust at $0.25 \mathrm{~m}$ (ground level for the experiment) while the other was placed at a higher point varying from $0.5 \mathrm{~m}$ to $2.0 \mathrm{~m}$ at $0.5 \mathrm{~m}$ increments $(0.5,1.0,1.5$, and $2.0 \mathrm{~m})$. Sampling was repeated three times at each height, except at $2.0 \mathrm{~m}$ where the sample was repeated four times. The experiment was repeated an extra time because of obtaining a ground $(0.25 \mathrm{~m})$ sample that appeared different (lower) than the others, all of the data were included in the analysis. The samplers were again set up to simultaneously take consecutive 20-second 
WHC-MR-0496

samples with the counts separated by a 40 -second hold time over a 8 to 14 minute time frame. The shorter times were typically used in the PUREX facility to minimize exposure to radiation. Data from these samples were entered into the Aerosol Particle Measurement Record where mean optical diameters were calculated.

Table 6 shows the largest mean optical diameter obtained by both laser particle counters being operated at the various heights selected at all three facilities. Each 7 ine of data shows pairs of simultaneously determined mean optical diameters, the value at $0.25 \mathrm{~m}$ and the value at the elevation indicated by that column. Table 6 also shows the mean and standard deviation for each height combination. The only significant variability was at $0.25 \mathrm{~m}$, near the floor, where it was difficult to "grab" the time of maximum particle size. 
Table 6. Peak Mean Diameter $(\mu \mathrm{m})$, Versus Sampling Height

Sampling Height

\begin{tabular}{|c|c|c|c|c|c|c|}
\hline Facility & & $0.25 \mathrm{~m}$ & $0.5 \mathrm{~m}$ & $1.0 \mathrm{~m}$ & $1.5 \mathrm{~m}$ & $2.0 \mathrm{~m}$ \\
\hline$\overline{3706 A}$ & $\therefore$ & $\begin{array}{r}10.06 \\
12.05 \\
9.64 \\
8.84 \\
11.59 \\
12.22 \\
8.02 \\
11.36 \\
11.41 \\
10.96 \\
5.34 \\
11.62 \\
8.71 \\
\end{array}$ & $\begin{array}{l}4.7 \\
5.56 \\
6.12\end{array}$ & $\begin{array}{l}1.87 \\
1.66 \\
1.67\end{array}$ & $\begin{array}{l}1.54 \\
2.18 \\
1.73\end{array}$ & $\begin{array}{l}2.17 \\
1.97 \\
2.01 \\
2.13\end{array}$ \\
\hline & $\begin{array}{l}\text { Mean } \\
\text { Standard } \\
\text { Deviation }\end{array}$ & $\begin{array}{c}10.14 \\
1.9\end{array}$ & $\begin{array}{l}5.46 \\
0.58\end{array}$ & $\begin{array}{l}1.73 \\
0.10\end{array}$ & $\begin{array}{l}1.82 \\
0.27\end{array}$ & $\begin{array}{l}2.07 \\
0.08\end{array}$ \\
\hline & $\begin{array}{l}\text { Coefficient } \\
\text { of Variation }\end{array}$ & $19 \%$ & $10 \%$ & $6 \%$ & $15 \%$ & $4 \%$ \\
\hline 3706, Rm. 102 & & $\begin{array}{l}3.60 \\
2.58 \\
4.20 \\
3.18 \\
\end{array}$ & 1.95 & 2.34 & 1.94 & 2.43 \\
\hline & $\begin{array}{l}\text { Mean } \\
\text { Standard } \\
\text { Deviation }\end{array}$ & $\begin{array}{l}3.39 \\
0.59\end{array}$ & 1.95 & 2.34 & 1.94 & 2.43 \\
\hline
\end{tabular}

\begin{tabular}{|c|c|c|c|c|c|c|}
\hline PUREX & & $\begin{array}{l}5.13 \\
3.73 \\
3.83 \\
4.73\end{array}$ & 1.49 & 1.22 & 0.56 & 0.57 \\
\hline & $\begin{array}{l}\text { Mean } \\
\text { Standard } \\
\text { Deviation }\end{array}$ & $\begin{array}{l}4.38 \\
0.61\end{array}$ & 1.49 & 1.22 & 0.57 & 0.56 \\
\hline
\end{tabular}

The largest of the mean optical diameters in the breathing zone was $2.07 \mu \mathrm{m}, 2.43 \mu \mathrm{m}$, and $1.22 \mu \mathrm{m}$ for the 3706A, 3706 and PUREX 
buildings respectively as shown in Table 6 . The breathing zone has been defined as above one meter for this study as workers may sit or lean to work. The mean of these three numbers is $1.9 \mu \mathrm{m}$ OD or $2.9 \mu \mathrm{m} \mathrm{AED.} \mathrm{To}$ be conservative, $4.0 \mu \mathrm{m}$ AED will be used.

The data for the 3706A facility from Table 6 are plotted in Fig. 3 to illustrate the variability in the ranges. The plot shows a flattening of data above $1 \mathrm{~m}$, the breathing zone. The plot also shows a trend toward greater variability in the observed data at low elevations. There was no correlation between the peak at $0.25 \mathrm{~m}$ and the pared peak at the higher sampling point. Additionally, a series of measurements of the ambient air at each height was made and is shown in the figure.

Figure 3 shows that even in situations where there is heavy dust loading, such as in the $3706 \mathrm{~A}$ facility, the mean optical diameter of the dust resuspended into the breathing zone is much less than the $10 \mu \mathrm{m}$ AED. 


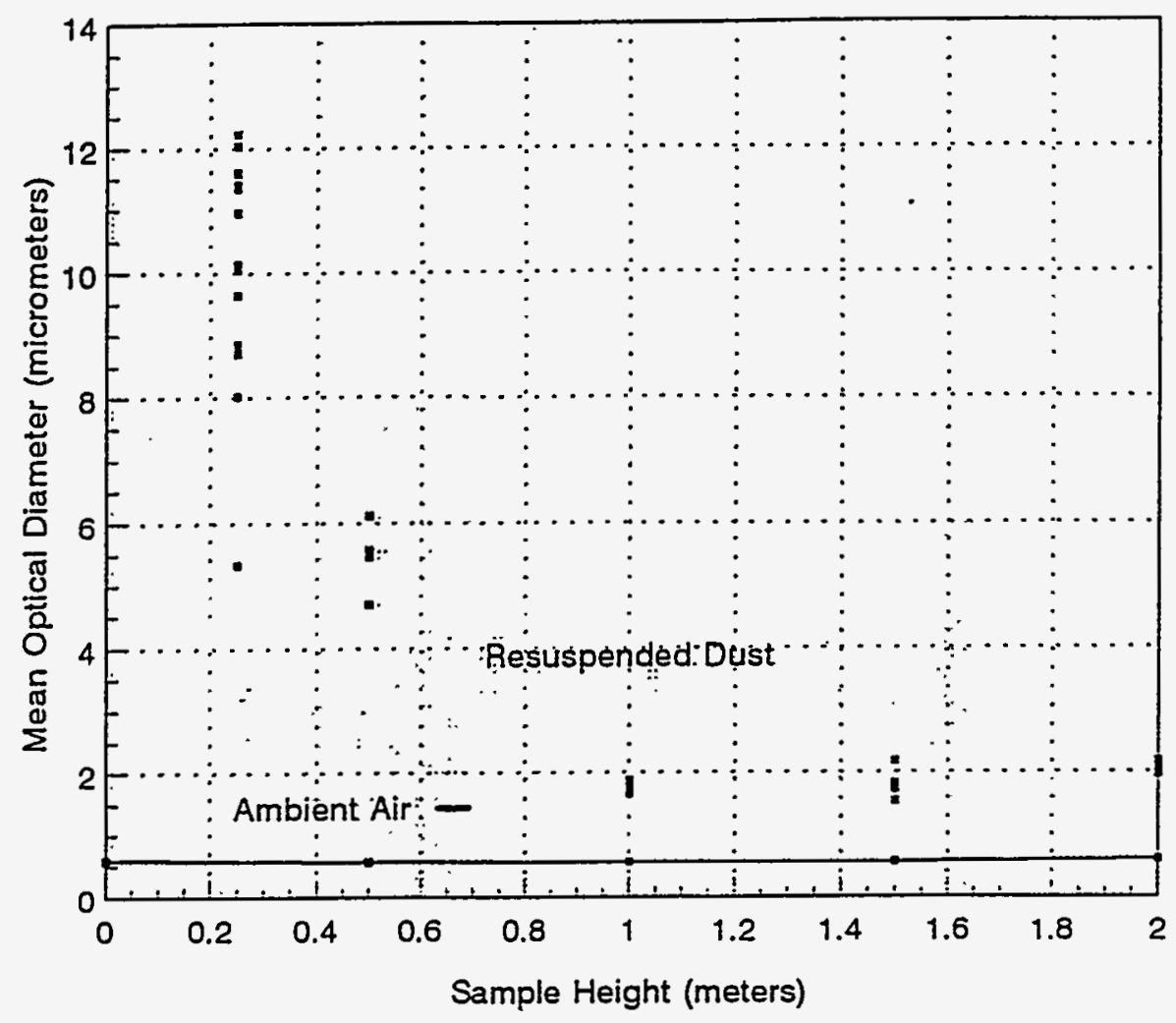

Fig. 3. Resuspension and Ambient Air Vs.: Sample Height, Bldg. 3706A.

To perform this experiment for 13 series of measurements required a significant amount of time and resulted in extensive data. To demonstrate similar results in the other buildings on the Hanford Site only one set of data was obtained at each height.

The same experiment was performed in the 3706 Building, room 102 . However, onty one dust resuspension measurement was performed at each height increment. The largest mean optical diameter of the resuspended dust and the largest mean diameter of the ambient air versus height is shown in Table 6 and are plotted in Fig. 4. These results are similar to those obtained at the $3706 \mathrm{~A}$ Building; however, the mean optical 
diameter at the $0.25 \mathrm{~m}$ sampling height was significantly lower, as was expected because it was a cleaner facility.

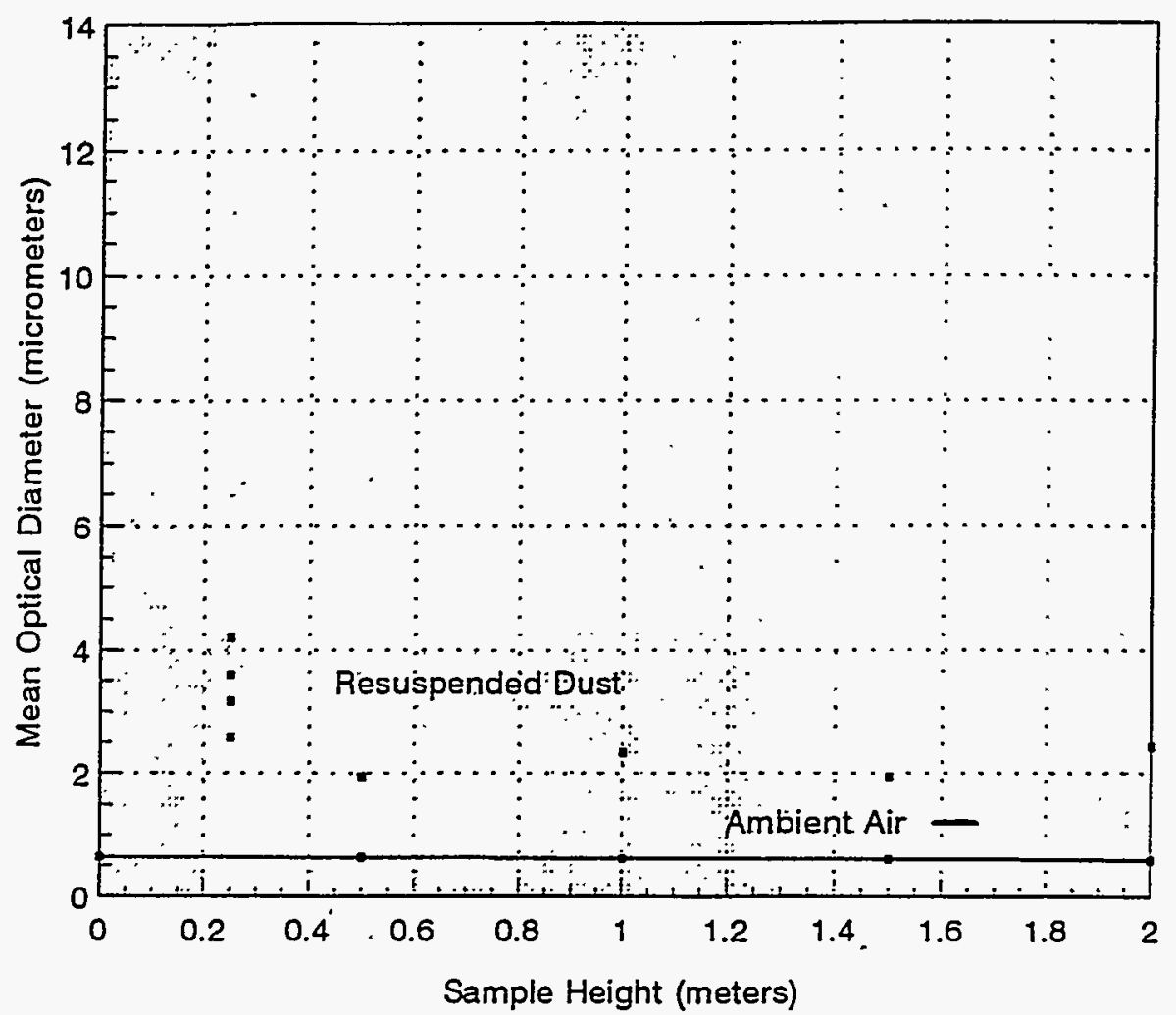

Fig. 4. Resuspension and Ambient Air Vs. Sample Height, 3706, Rm. 102

The protocol used for the 3706 Building room 102 was also used in the PUREX facility. The largest mean optical diameter of the resuspended dust and the largest mean optical diameter of ambient air versus height is shown in Table 6 and is plotted in Fig. 5. The mean optical diameter of the dust resuspended into the breathing zone is less than, but consistent with, the findings at the other two facilities. There was inconsistency in how many particles were resuspended from 
sample to sample and in the maximum particle size near the floor; however, trends observed above one meter were consistent.

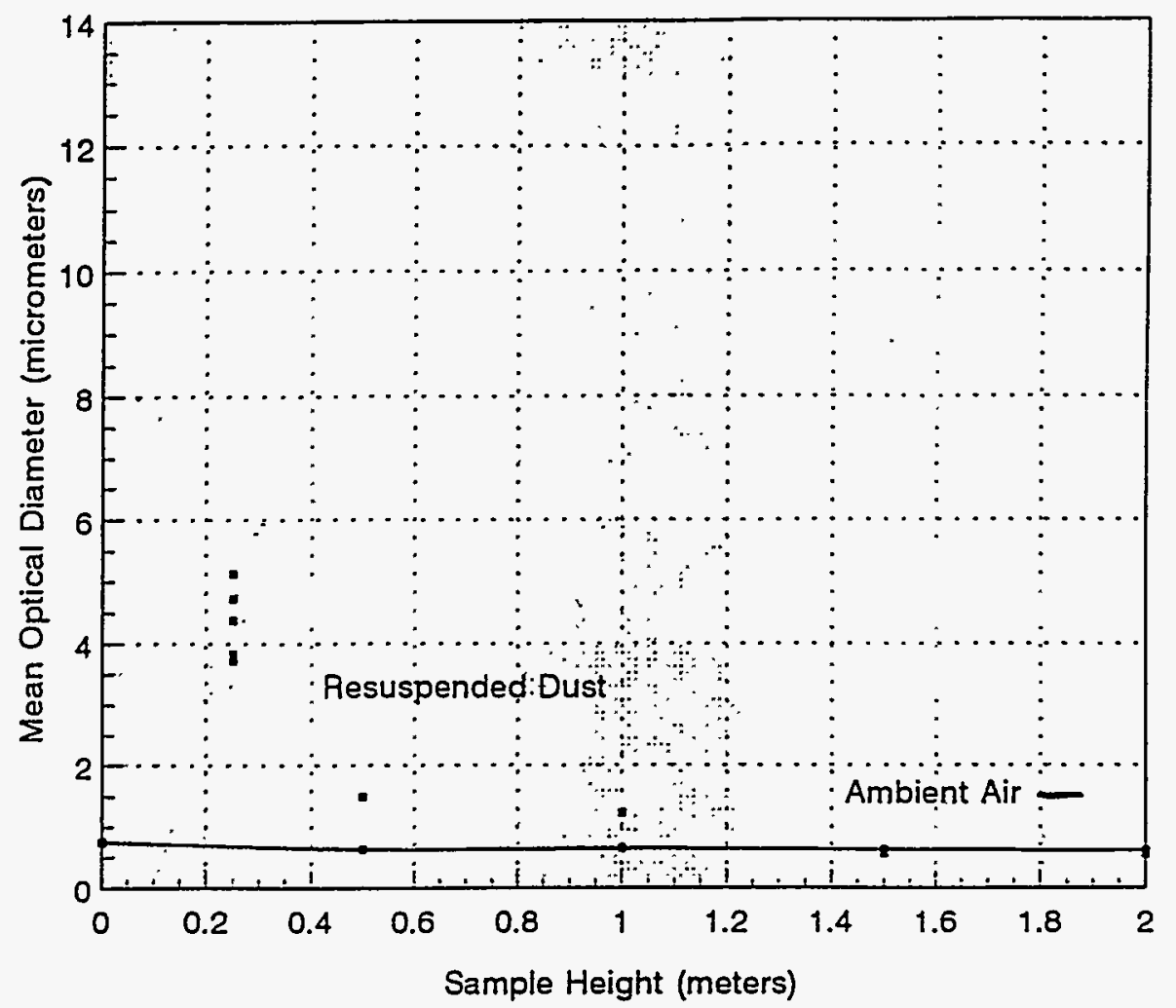

Fig. 5. Resuspension and Ambient Air Vs. Sample Height, PUREX.

One possible explanation for the smaller particle size above one meter at the PUREX facility could be the depth within the building of the pipe and operating gallery ( $P \& 0)$ where the samples were taken. There are three barriers for the dust entering from outside the building to pass through to get to the P\&O gallery as opposed to one or two in the previous facilities. Larger particles would be less likely to get there than small ones because of deposition on doorjambs as the air moves around the bends created by the doorways. 
WHC-MR-0496

Analysis

The percent of large particles in the breathing zone was analyzed by calculating the percent of the total count-weighted size for the bins with mean optical diameter greater than $3.16 \mu \mathrm{m}$ as shown in the data sheets, appendix $H$. Descending cumulative percents were also shown for these bins. These data are shown in Tables 7 through 9. 
Table 7. Percent of Large Particles in Breathing Zone, Bildg. 3706A

\begin{tabular}{|c|c|c|c|c|c|}
\hline & $\begin{array}{l}\text { Highest } \\
\text { Mean } \\
\text { Diameter }\end{array}$ & 1.87 & & & \\
\hline Height & & $\begin{array}{l}\text { Bin Mean } \\
\text { Diameter } \\
(\mu \mathrm{m})\end{array}$ & Count & $\%$ & $\begin{array}{c}\text { Descending } \\
\text { Cumulative } \\
\%\end{array}$ \\
\hline \multirow[t]{2}{*}{$1.0 \mathrm{~m}$} & & $\begin{array}{c}3.16 \\
7.07 \\
14.14 \\
\text { Total a11 } \\
\text { Counts }\end{array}$ & $\begin{array}{r}70548 \\
5456 \\
2573 \\
232450\end{array}$ & $\begin{array}{c}30 \\
2.3 \\
1.1\end{array}$ & $\begin{array}{c}34 \\
3.4 \\
1.1\end{array}$ \\
\hline & $\begin{array}{l}\text { Highest } \\
\text { Mean : } \\
\text { Diameter }\end{array}$ & 2.18 & & & \\
\hline \multirow[t]{2}{*}{$1.5 \mathrm{~m}$} & & $\begin{array}{c}3.16 \\
7.07 \\
14.14 \\
\text { Total al1 } \\
\text { Counts }\end{array}$ & $\begin{array}{r}508553 \\
6296 \\
7569 \\
193884\end{array}$ & $\begin{array}{l}26 \\
3.2 \\
3.9\end{array}$ & $\begin{array}{c}33 \\
7.1 \\
3.9\end{array}$ \\
\hline & $\begin{array}{l}\text { Highest } \\
\text { Mean } \\
\text { Diameter }\end{array}$ & 2.17 & & & \\
\hline $2.0 \mathrm{~m}$ & & $\begin{array}{c}3.16 \\
7.07 \\
14.14 \\
\text { Total al1 } \\
\text { Counts } \\
\end{array}$ & $\begin{array}{r}54338 \\
6253 \\
7752 \\
204896\end{array}$ & $\begin{array}{r}27 \\
3.1 \\
1.3\end{array}$ & $\begin{array}{r}31 \\
4.4 \\
1.3\end{array}$ \\
\hline Mean & & $\begin{array}{r}3.16 \\
7.07 \\
14.14\end{array}$ & & & $\begin{array}{r}33 \\
5.0 \\
2.1\end{array}$ \\
\hline
\end{tabular}


Table 8. Percent of Large Particles in Breathing Zone, 3706, Rm. 102

\begin{tabular}{|c|c|c|c|c|c|}
\hline & $\begin{array}{l}\text { Highest } \\
\text { Mean } \\
\text { Diameter }\end{array}$ & 2.34 & & & \\
\hline Height & & $\begin{array}{l}\text { Bin Mean } \\
\text { Diameter } \\
(\mu \mathrm{m})\end{array}$ & Count & $\%$ & $\begin{array}{c}\text { Descending } \\
\text { Cumulative } \\
\%\end{array}$ \\
\hline$\overline{1.0 m}$ & & $\begin{array}{l}3.16 \\
7.07 \\
14.14 \\
\text { Total a11 } \\
\text { Counts }\end{array}$ & $\begin{array}{r}17134 \\
3842 \\
3258 \\
66897\end{array}$ & $\begin{array}{r}26 \\
5.7 \\
4.9 \\
\end{array}$ & $\begin{array}{c}37 \\
11 \\
4.9\end{array}$ \\
\hline
\end{tabular}

\begin{tabular}{|c|c|c|c|c|c|}
\hline & $\begin{array}{l}\text { Highest } \\
\text { Mean } \\
\text { Diameter }\end{array}$ & 1.94 & & & \\
\hline $1.5 \mathrm{~m}$ & & $\begin{array}{l}3.16 \\
7.07 \\
14.14 \\
\text { Total a11 } \\
\text { Counts }\end{array}$ & $\begin{array}{r}11529 \\
2654 \\
1857 \\
54395\end{array}$ & $\begin{array}{r}21 \\
4.9 \\
3.4\end{array}$ & $\begin{array}{r}29 \\
8.3 \\
3.4\end{array}$ \\
\hline
\end{tabular}

\begin{tabular}{|c|c|c|c|c|c|}
\hline & $\begin{array}{l}\text { Highest } \\
\text { Mean } \\
\text { Diameter }\end{array}$ & 2.43 & & & \\
\hline $2.0 \mathrm{~m}$ & & $\begin{array}{l}3.16 \\
7.07 \\
14.14 \\
\text { Total al1 } \\
\text { Counts }\end{array}$ & $\begin{array}{r}14828 \\
3760 \\
2848 \\
56427\end{array}$ & $\begin{array}{r}26 \\
6.7 \\
5.0\end{array}$ & $\begin{array}{l}38 \\
12 \\
5.0\end{array}$ \\
\hline Mean & & $\begin{array}{r}3.16 \\
7.07 \\
14.14\end{array}$ & & & $\begin{array}{l}35 \\
10 \\
4.4\end{array}$ \\
\hline
\end{tabular}


Table 9. Percent of Large Particles in Breathing Zone, PUREX

\begin{tabular}{|c|c|c|c|c|c|}
\hline & $\begin{array}{l}\text { Highest } \\
\text { Mean } \\
\text { Diameter }\end{array}$ & 1.22 & & & \\
\hline Height & & $\begin{array}{l}\text { Bin Mean } \\
\text { Diameter } \\
(\mu \mathrm{m})\end{array}$ & Count & $\cdot$ & $\begin{array}{c}\text { Descending } \\
\text { CumuTative } \\
\%\end{array}$ \\
\hline \multirow[t]{2}{*}{$\overline{1.0 \mathrm{~m}}$} & & $\begin{array}{l}3.16 \\
7.07 \\
14.14 \\
\text { Total all } \\
\text { Counts }\end{array}$ & $\begin{array}{r}8045 \\
753 \\
242 \\
50994\end{array}$ & $\begin{array}{l}16 \\
1.5 \\
0.47\end{array}$ & $\begin{array}{l}18 \\
2.0 \\
0.47\end{array}$ \\
\hline & $\begin{array}{l}\text { Highest } \\
\text { Mean } \\
\text { Diameter }\end{array}$ & 0.57 & & & \\
\hline \multirow[t]{2}{*}{$1.5 \mathrm{~m}$} & & $\begin{array}{c}3.16 \\
7.07 \\
14.14 \\
\text { Total al1 } \\
\text { Counts }\end{array}$ & $\begin{array}{r}658 \\
51 \\
31 \\
41532\end{array}$ & $\begin{array}{l}1.6 \\
0.12 \\
0.07\end{array}$ & $\begin{array}{l}1.8 \\
0.19 \\
0.07\end{array}$ \\
\hline & $\begin{array}{l}\text { Highest } \\
\text { Mean } \\
\text { Diameter }\end{array}$ & 0.56 & & & \\
\hline $2.0 \mathrm{~m}$ & & $\begin{array}{c}3.16 \\
7.07 \\
14.14 \\
\text { Total a } 71 \\
\text { Counts } \\
\end{array}$ & $\begin{array}{r}411 \\
55 \\
101 \\
46937\end{array}$ & $\begin{array}{l}0.88 \\
0.12 \\
0.22\end{array}$ & $\begin{array}{l}1.2 \\
0.34 \\
0.22\end{array}$ \\
\hline$\overline{\overline{M e a n}}$ & & $\begin{array}{r}3.16 \\
7.07 \\
14.14\end{array}$ & & & $\begin{array}{l}7.0 \\
0.84 \\
0.25\end{array}$ \\
\hline
\end{tabular}

Table 10. Mean Percent of Large Particles in Breathing Zone

\begin{tabular}{ccc}
$\begin{array}{c}\text { Bin Mean } \\
\text { Diameter } \\
(\mu \mathrm{m})\end{array}$ & $\begin{array}{l}\text { Bin Minimum } \\
\text { Diameter } \\
(\mu \mathrm{m})\end{array}$ & $\begin{array}{c}\text { Descending } \\
\text { Cumulative } \\
\%\end{array}$ \\
\hline 3.16 & 2.00 & 25 \\
7.07 & 5.00 & 5.3 \\
14.14 & 10.0 & 2.3
\end{tabular}


The mean data from Tables 7,8 , and 9 were averaged together to calculate the size distribution in the breathing zone. This indicates that, on average, $25 \%$ of the particles, by number, are of diameters greater than a minimum size of $2.00 \mu \mathrm{m}$ OD or a mean size of $3.16 \mu \mathrm{m} \mathrm{OD}$, while $5.3 \%$ are greater than a minimum size of $5.00 \mu \mathrm{m}$ OD or a mean size of $7.07 \mu \mathrm{m} \mathrm{OD}$, and $2.3 \%$ are of diameters greater than a minimum size of $10.0 \mu \mathrm{m} 0 \mathrm{D}$ or a mean size of $14.14 \mu \mathrm{m} \mathrm{OD}$. There was a relatively wide variation around these means.

The ambient air has a mean diameter of $0.62 \mu \mathrm{m}$ OD as shown in Chapter II. Although, $25 \%$ of the breathing air particles have a size greater than more than 3 times $(2.0 \div 0.62)$ that of ambient air, only slightly over $5 \%$ are greater than eight $(8)$ times $(5.0 \div 0.62)$ this. Only 2.3\% are greater than $10 \mu \mathrm{m}$ OD as shown in Table 10.

The $5.0 \mu \mathrm{m}$ OD is equivalent to a $7.5 \mu \mathrm{m} \mathrm{AED}$, thus less than $5 \%$ of the particles in the breathing zone are greater than the $10 \mu \mathrm{m}$ AED recommended by the Nuclear Regulatory Commission (NRC) as a default value for airborne particulate line loss studies.

Regression analyses were conducted on the mean optical diameters as a function of the sampling height for each facility. Logarithmic, exponential, and power function regressions were determined along with their accompanying coefficients of determination $\left(r^{2}\right)$ as shown in Table 11. The logarithmic function appeared to best represent the resuspension of dust and is illustrated in the respective facility graphs (Figs $3,4, \& 5$ ). The combined (i.e., al1 three facilities together) function was; 


$$
d=2.4-(2.1 * \ln h) \quad\left[r^{2}=0.691\right]
$$

where $d$ is the mean optical diameter $(\mu \mathrm{m})$ and $h$ is the corresponding sampling height (m).

Table 11. Mean Optical Diameter, d $(\mu \mathrm{m})$, as a Function of Sampling Height, $h(m)$, and Coefficient of Determination $\left(r^{2}\right)$

Facility

$3706 \mathrm{~A}$

3706

PUREX

Regression

Logarithmic,
$\mathrm{d}=$
$3.45-4.03 * \ln \mathrm{h}$
$2.33-0.43 * 7 n h$
$1.31-1.71 * 7 \mathrm{n} \mathrm{h}$
$r^{2}=$
0.872
0.371
0.830

Exponential, d

$\begin{array}{llll}= & 8.39 * \mathrm{e}^{-0.904 \mathrm{~h}} & 2.67 * \mathrm{e}^{-0.119 h} & 3.75 * \mathrm{e}^{-1.08 \mathrm{~h}} \\ r^{2}= & 0.671 & 0.141 & 0.837\end{array}$

Power

Function, $d=2.74 * h^{-0.871}$

$2.29 * h^{-1.51}$

$0.996 * h^{-0.971}$

$r^{2}=$

0.865

0.312

0.941 
Comparisons were made between the indoor ambient airborne particulate size distribution and that of resuspended settled dust in facilities at the Hanford Site that represent buildings that will undergo routine decommissioning operations. The results indicate that Tess than $5 \%$ of the particles, by count, above the breathing zone are greater than $10, \mu \mathrm{m}$ AED in size, (Table 10), and the breathing zone has a mean aerodynamic equivalent diameter (AED) of $4 \mu \mathrm{m}$ or less, (summary of Table 6).

This is less than or equal to the values previously developed for use in line loss studies at the Hanford Site and less than the $10 \mu \mathrm{m}$ conservative default value used by the Nuclear Regulatory Commission (NRC) for airborne particulate sample line loss determinations.

It is recommended that a 4- $\mu$ m AED particle size default value be used during decommissioning operations. 


\section{REFERENCES}

Alzona, J.; Cohen, B. L.; Rudolph, H.; Jow, H. N.; Frohtiger, J. 0. Indoor-Outdoor Relationships For Airborne Particulate Matter of Outdoor Origin. Atmospheric Environment 13:55-60; 1979.

American Conference of Governmental Industrial Hygienists. Particle Size Selective Sampling in the Workplace. Cincinnati. ACGIH 1985.

American National Standard. Guide to Sampling Airborne Radioactive Materials in Nuclear Facilities; ANSI N13.1; 1969.

Anand, N. K.; McFarland, A. R.; McGuire, S. A. Deposition: Software to Calculate Particle Penetration Through Aerosol Transport Lines. NUREG/GR-0006 (Draft); 1991.

Anand, N. K.; McFarland, A. R.; Wong, F. S.; Kocmoud, C. J. Optimization of Aerosol Penetration Through Transport Lines. Aerosol Technology Laboratory Report 6441/01/31/93/NKA; 1993.

Andersen, B. V.; Nelson, I. C. Plutonium Air Concentrations and Particle Size Relationships in Hanford Facilities. Pacific Northwest Laboratory BNWL-495; 1967. 
WHC-MR-0496

Andersen Samplers Inc. Series 210 Ambient Cascade Impactor and Cyclone Preseparator Users Manual. Atlanta, Bu7letin 579-20IM; 1979.

Benjamin Y. H. L.; Berglund R. N.; Agarwal J. K. Experimental Studies of Optical Particle Counters. Atmospheric Environment. 8:717-732; 1974.

Caplan, K. J. The Significance of Wipe Samples. American Industrial Hygiene Association Journa7. AIHAJ 54:(2)70-75; 1993.

Chen B. T.; Cheng Y. S. Optical Diameters of Aggregate Aerosols. Journal Aerosol Science, 15:615-623; 1984.

Fan, B. J.; Wong, F. S.; McFarland, A. R.; Anand, N. K. Aerosol Deposition in Sampling Probes. Aerosol Science and Technology. $17: 326-332 ; 1992$.

Fish, B. R. Surface Contamination. New York: Pergamon Press; 1964.

Glissmeyer, J. Recommendation of Particle Line Loss Code. Pacific Northwest Laboratory; PNL-8037; 1991.

Gouesbet G.; Grehan G. Optical Particle Sizing: Theory and Practice. New York: Plenum Press; 1988. 
Hickey E. E.; Stoetzel G. A.; Strom D. J.; Cicotte G. R.; Wiblin C. M.; McGuire S. A. Air Sampling in the Workplace. NUREG-1400; 1993.

Hinds W. C. Aerosol Technology. Properties, Behavior, and Measurement of Airborne Particles. New York: John Wiley \& Sons; 1982.

International Commission of Radiological Protection, Reference Man. Oxford, Pergamon Press. ICRP 23; 1975.

Killinger R. T.; Zerull R. H. Effects of Shape and Orientation to be Considered for Optical Particle Sizing. 419-429; 1984.

Liu, B.Y.H. Basic Aerosol Properties and Behavior. University of Minnesota, Training handout; 1991.

Marple V. A.; Rubow K. L. Aerodynamic Particle Size Calibration of Optical Particle Counters. J. Aerosol Sci. 7:425-433; 1976.

McFarland, A. R.; Wong, F. S.; Anand, N. K.; Ortiz, C. A. Aerosol Penetration Through a Model Transport System: Comparison of Theory and Experiment. Environmental Science \& Technology. 25:1573-1577; 1991.

Met One, Inc. Owners Manual Model 200L laser Particle Counter; 1989. 
Owen M. K.; Ensor D. S.; Sparks L. E. Airborne Particle Sizes and Sources Found in Indoor Air. Atmospheric Environment. 26A:2149-2162; 1992.

Perrin, M. L. Plutonium Aerosol Size Distributions in a Reprocessing Plant and During Decommissioning Operations. Proceedings of the Department of Energy Workshop on Workplace Aerosol Monitoring. Pacific . Northwest Laboratory; 1987.

Radiological Health Handbook (RHH). PB-230846. U. S. Dept. HEW; 1970.

Rathbun, L. A. Particle Size Assigned to Airborne Radioactivity in Westinghouse Hanford Facilities Containing Mixed Fission Products. WHC 33610-92-LAR-013; I992.

Ruben, R. H. Characterization of Mass and Activity Distributions in a Cascade Impactor Sample from B-Plant Canyon, Cell 9. WHC 33610-92-RHR-013; 1992a.

Ruben, R. H. Particle Size Assigned to Airborne Radioactivity in Westinghouse Hanford Facilities Containing Plutonium Compounds. WHC 33610-92-RHR-010; $1992 \mathrm{~b}$.

Sehme1, G. A. Literature Ranges of Wind and Mechanically Caused Resuspension Factors. PNL Annual Report to DOE, 102-107; 1979. 
WHC-MR-0496

Strenge, D. L.; Kennedy, R. A.; SuTa, M. J.; Johnson, J. R. Code for Internal Dosimetry (CINDY ver. 1.2), Part 1: Conceptual Representation, PNL-7493, Pt. 1, rev. I; 1992.

Sula, M. J.; Carbough, E. H.; Bihl, D. E. Technical Basis for Internal Dosimetry at Hanford. Pacific Northwest Laboratory. PNL-6866; 1989.

U. S. Nuclear Regulatory Commission. Draft Regulatory Guide. Deposition in Sampling Lines. DG-8003; 1991.

U. S. Nuclear Regulatory Commission. Regulatory Guide $8.25 \mathrm{Rev}$. Air Sampling in the Workplace; 1992.

Yu, C. C.; Tung, C. J.; Hung, I. F.; Tseng, C. L. Anatyses of Radioactive Aerosols to support Accurate Internal Dose Assessments At Chinshen Nuclear Power Plant. Health Physics. 65:147-155; 1993. 
Appendix A

\section{AED CONVERSION FROM OD}

AED, Aerodynamic Equivalent Diameter

OD, Optical Diameter

$\mathrm{Pp}$, Density of particle of interest

Po, Reference particle density, $1 \mathrm{~g} / \mathrm{cm}^{3}$

C, Cunningham slip correction for sphere of interest, Dust

Ca, Cunningham slip correction for sphere of radius a

1) $\mathrm{AED} / \mathrm{OD}=(\mathrm{Pp} / \mathrm{Po})^{0.5}(\mathrm{C} / \mathrm{Ca})^{0.5}$ (Liu, 1991)

For this study the particle size of interest is from 3 to $10 \mu \mathrm{m}$ in size.

2) $\mathrm{C}=1$ for particles above approximately $3 \mu \mathrm{m}$ (Liu, 1991) 
Substituting;

3) $\mathrm{AED} / \mathrm{OD}=(\mathrm{Pp} / \mathrm{Po})^{0.5}$

For this study it will be assumed that the main constituent of dust is dirt.

4) $\mathrm{Pp}$ is approximately $2.3 \mathrm{~g} / \mathrm{cm}^{3}$ (RHH, 1970)

Substituting;

5) $\mathrm{AED} / \mathrm{OD}=(2.3)^{0.5}=1.5$ or $\mathrm{AED}=1.5 \mathrm{OD}$

This is the same conversion factor reported by Marple and Rubow (1976). 
Appendix B

\section{INSTRUCTIONS FOR OPERATION OF LASER PARTICLE COUNTERS}

1. Attach air pump via TYGON@* tubing to rear of Laser Particle Counter, LPC where it is labeled "RETURN AIR".

2. Attach sample probe via TYGON@ tubing to intake port on top of LPC.

3. Plug in power cord.

4. Press "ON".

5. Press "AUDIO ALARM" to stop alarm. The printer should enable itself but if not press "OPTION" then "I" then "ENTER".

6. Set time by pressing "TIME" then "PROG" then set the time using no spaces; ex. 084500 for 8:45, then press "ENTER".

7. Set date by pressing "DATE" then "PROG" then set data; ex. 092292 for September 22, 1992, then press "ENTER".

*Tygon is a trademark of the Dupont Company, Wilmington, Delaware. 
8. Press "SAMPLE TIME", then "PROG", then set whatever time is necessary; ex. 60 for 1 minute, then press "ENTER".

9. Press "HOLD TIME", then "PROG", then set whatever time; ex. 05 this is the time between counts, then press "ENTER".

10. Press "COUNT", then "ALARM LIMIT HIGH". The particle size should display "0.3", press "PROG", then press "ENTER". Next press the

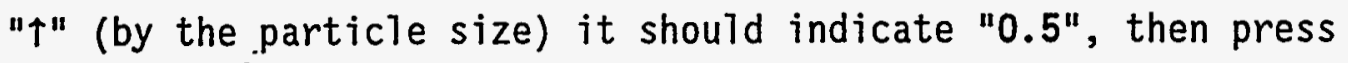
"PROG", then press "ENTER". Continue pressing "†" until all particle size ranges are programmed.

11. Press "MODE" to change from "MANUAL" to "AUTO".

12. Press "COUNT MODE" to change from "CUM" to "DIFF".

13. Make sure the "MODE" "LOCAL" is lit instead of "REMOTE".

14. Press "COUNT".

15. Press "OPER" and wait for the center green indicator to light. Adjust air flow as needed. If this internal calibration cannot $\therefore$ be adjusted to a green light (OK), DO NOT USE this counter at this time. 
16. Press "RUN" and count should start changing. All particle sizes can be checked by pressing " $\uparrow "$.

17. Wait unti1 the LPC has printed out at least twice to make sure everything is running properly.

\section{INSTRUCTION FOR SHUTTING DOWN LASER PARTICLE COUNTERS}

1. Press "STOP".

2. Press "CALIB".

3. If the paper printout is desired, press "PAPER FEED" and tear off.

4. Press "OFF". 
Appendix C

\section{Aerosol Particle Measurement Record}

Description of sample location

Date

Time sample started

Temperature Humidity Dew Point

Average Wind Velocity (if applicable) \# of physical barriers between sample area and outside (if applicable)

Sample \#1

\begin{tabular}{|c|c|c|c|c|}
\hline $\begin{array}{l}\text { Bin } \\
\text { Range } \\
(\mu \mathrm{m})\end{array}$ & $\mid \begin{array}{l}\text { Bin Mean } \\
\text { Diameter } \\
(\mu \mathrm{m})\end{array}$ & $\begin{array}{l}\text { Bin } \\
\text { Width }\end{array}$ & Count & $\begin{array}{l}\text { Weighted } \\
\text { Mean } \\
\text { Diameter }\end{array}$ \\
\hline $\begin{array}{l}0.30- \\
0.49\end{array}$ & 0.38 & 0.21 & & \\
\hline $\begin{array}{l}0.50- \\
0.99\end{array}$ & 0.70 & 0.30 & & \\
\hline $\begin{array}{l}1.00- \\
1.99\end{array}$ & 1.41 & 0.30 & & \\
\hline $\begin{array}{r}2.00- \\
4.99\end{array}$ & 3.16 & 0.40 & & \\
\hline $\begin{array}{l}5.00 \\
9.99\end{array}$ & 7.07 & 0.30 & & \\
\hline$>10.0$ & 14.14 & 0.30 & & \\
\hline
\end{tabular}

Sample \#2

\begin{tabular}{||r|l|l|l|l||}
\hline $\begin{array}{r}\text { Bin } \\
(\mu \mathrm{m})\end{array}$ & $\begin{array}{l}\text { Bin Mean } \\
\text { Diameter } \\
(\mu \mathrm{m})\end{array}$ & $\begin{array}{l}\text { Bin } \\
\text { Width }\end{array}$ & $\begin{array}{l}\text { Count } \\
\text { Mean } \\
\text { Diameter }\end{array}$ \\
\hline $\begin{array}{r}0.30-49 \\
0.50-\end{array}$ & 0.38 & 0.21 & & \\
0.99 & 0.70 & 0.30 & \\
\hline $\begin{array}{r}1.00- \\
1.99\end{array}$ & 1.41 & 0.30 & \\
\hline $2.00-$ & 3.16 & 0.40 & \\
\hline 4.99 & & & \\
\hline $5.00-$ & 7.07 & 0.30 & \\
9.99 & & & \\
\hline$>10.0$ & 14.14 & 0.30 & \\
\hline
\end{tabular}

In the field fill out the appropriate information and attach the Laser Particle Counter data print out to this form. Then enter all data into the respective blanks on a WP51 file of this form found on $\mathrm{J}: \mathrm{ll}$ ineloss.hje. To calculate the size weighted count, and the size mean diameter type ALT F7 Table Edit, Option 5 Math, and Option 1 Calculate. 
Appendix D

\section{INSTRUCTIONS FOR ASSEMBLY AND OPERATION OF THE CASCADE IMPACTOR}

1. Wash al1 parts thoroughty.

2. Remove the two thumb screws on the cylindrical inlet section. Lift off the cylindrical inlet section making sure that the nylon washers are in place.

3. Lift off the stages. Place the back-up filter on top of the stainless filter.

4. Put on stage labeled "F".

5. Place the \#10 substrate (rough side up) on the "F" stage so that the slots in the substrate match up with the slots in the stage. Place the "10" stage on top.

6. Place the \#9 substrate on the "10" stage again lining up the slots. Continue until all the stages are on.

7. There will be no substrate on top of the "1" so place the cylindrical inlet section on top of " 1 " followed by the thumb screws. (Make sure that the nylon washers are on and the thumb 
screws are tightened at the same time to insure that the 0-rings are not ruined.)

8. The cyclone preseparator should be on top of the cylindrical inlet section; if not, place it there.

9. Connect the cascade impactor to the constant flow sampler pump via Tygon ${ }^{\circledR}$ tubing.

10. Plug in sampler pump and reset the built-in timer.

11. Turn on sampling pump and set to the desired flow rate.

Note: Obtaining the filter dry weight is the most critical step.

\section{INSTRUCTION FOR DISASSEMBLY OF THE CASCADE IMPACTOR}

1. Remove the two thumb screws on the cylindrical inlet section. Lift off the cylindrical inlet section making sure that the nylon washers are in place.

2. Remove the substrates making sure that the order of the substrate remains correct. 


\section{Appendix F}

\section{CASCADE IMPACTOR SUBSTRATES}

Building 3706 Room 102

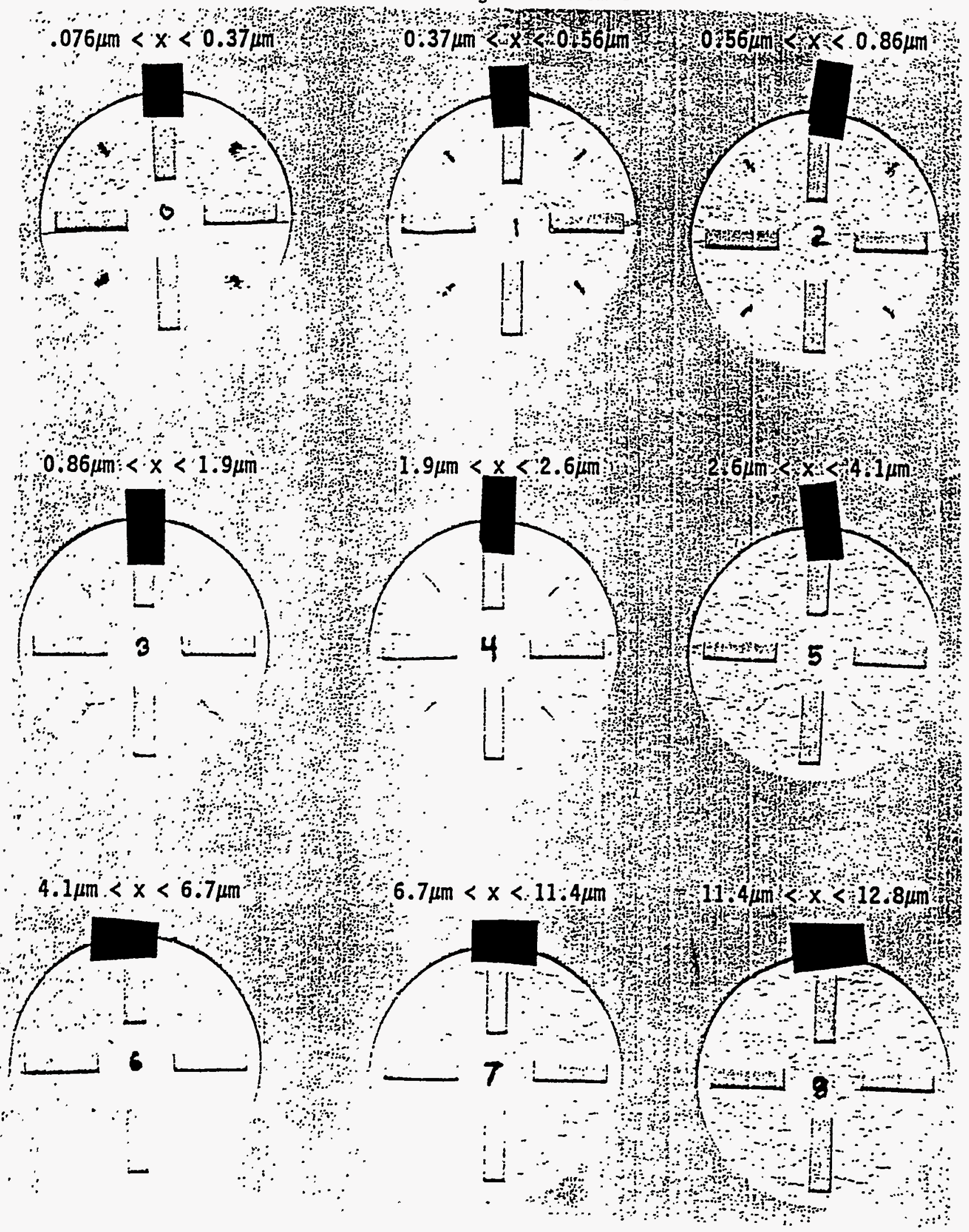


WHC-MR-0496

Appendix G

LASER PARTICLE COUNTER

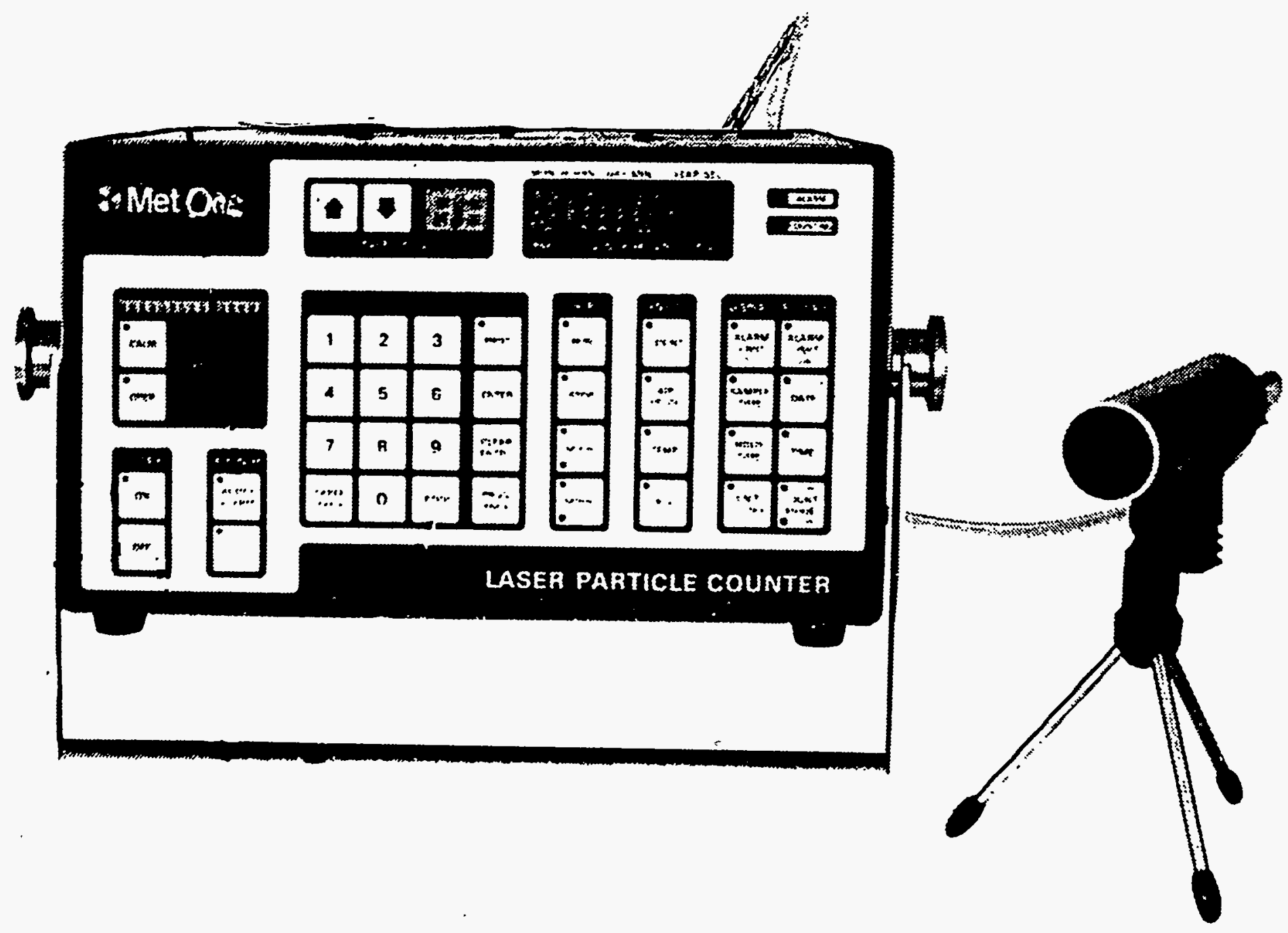


Appendix $\mathrm{H}$

AEROSOL PARTICLE MEASUREMENT RECORD DATA SHEETS 
Data for Table 2. Precision of the Laser Particle Counter

Sampler \#1

Sampler \#2 


\section{Aerosol Particle Measurement Record}

Description of sample location 3706 Building, Room 102, Comparison Test, Sampler \#1, Date $7 / 22 / 94$, Time sample started 13:26:56, Temperature 81.6 F, Humidity $39.3 \%$, Dew Point $55.6 \mathrm{~F}$, Average Wind Velocity (if applicable) $.08 \mathrm{~m} / \mathrm{s}$, \# of physical barriers between sample area and outside (if applicable). $\underline{3}$,

Sample \#1

\begin{tabular}{|c|c|c|c|c|}
\hline \begin{tabular}{|l} 
Bin \\
Range \\
(um)
\end{tabular} & \begin{tabular}{|l|} 
Bin Mean \\
Diameter \\
$(\mu \mathrm{m})$
\end{tabular} & $\begin{array}{l}\text { Diff. of the } \\
\text { log of the } \\
\text { Size }\end{array}$ & Counts & $\begin{array}{l}\text { Weighted } \\
\text { Mean } \\
\text { Diameter }\end{array}$ \\
\hline $0.30-0.49$ & 0.38 & 0.21 & 140301 & \multirow{6}{*}{0.64} \\
\hline $0.50-0.99$ & 0.70 & 0.30 & 39498 & \\
\hline $1.00-1.99$ & 1.41 & 0.30 & 8664 & \\
\hline $2.00-4.99$ & 3.16 & 0.40 & 6682 & \\
\hline $5.00-9.99$ & 7.07 & 0.30 & 529 & \\
\hline$>10.00$ & 14.14 & 0.30 & 566 & \\
\hline \multicolumn{5}{|l|}{ Sample \#3 } \\
\hline \begin{tabular}{|l} 
Bin \\
Range \\
$(\mu \mathrm{m})$
\end{tabular} & \begin{tabular}{|l|} 
Bin Mean \\
Diameter \\
$(u m)$
\end{tabular} & $\begin{array}{l}\text { Diff. of the } \\
\log \text { of the } \\
\text { Size }\end{array}$ & Counts & \begin{tabular}{|l} 
Weighted \\
Mean \\
Diameter
\end{tabular} \\
\hline $0.30-0.49$ & 0.38 & 0.21 & 141861 & \multirow{6}{*}{0.63} \\
\hline $0.50-0.99$ & 0.70 & 0.30 & 38990 & \\
\hline $1.00-1.99$ & 1.41 & 0.30 & 8121 & \\
\hline $2.00 \cdot 4.99$ & 3.16 & 0.40 & 5931 & \\
\hline $5.00-9.99$ & 7.07 & 0.30 & 420 & \\
\hline$>10.00$ & 14.14 & 0.30 & 577 & \\
\hline \multicolumn{5}{|l|}{ Sample \#5 } \\
\hline \begin{tabular}{|l} 
Bin \\
Range \\
$(\mu \mathrm{m})$
\end{tabular} & $\begin{array}{l}\text { Bin Moan } \\
\text { Diameter } \\
(\mu \mathrm{m})\end{array}$ & $\begin{array}{l}\text { Diff. of the } \\
\log \text { of the } \\
\text { Size }\end{array}$ & Counts & $\begin{array}{l}\text { Weighted } \\
\text { Mean } \\
\text { Diameter }\end{array}$ \\
\hline \begin{tabular}{|c|c|}
$0.30-0.49$ \\
\end{tabular} & $\begin{array}{l}0.38 \\
\end{array}$ & 0.21 & 144523 & \multirow{6}{*}{0.59} \\
\hline $0.50 \cdot 0.99$ & 0.70 & 0.30 & 39045 & \\
\hline $1.00-1.99$ & 1.41 & 0.30 & 7711 & \\
\hline $2.00-4.99$ & 3.16 & 0.40 & 5117 & \\
\hline $5,00-9.99$ & 7.07 & 0.30 & 371 & \\
\hline$>10.00$ & 14.14 & 0.30 & 307 & \\
\hline \multicolumn{5}{|l|}{ Sample \#7 } \\
\hline $\begin{array}{l}\text { Bin } \\
\text { Range } \\
\text { (um) }\end{array}$ & \begin{tabular}{|l|} 
Bin Mean \\
Diameter \\
$(\mu \mathrm{m})$
\end{tabular} & $\begin{array}{l}\text { Diff. of the } \\
\text { log of the } \\
\text { Size }\end{array}$ & Counts & $\begin{array}{l}\text { Weighted } \\
\text { Mean } \\
\text { Diameter }\end{array}$ \\
\hline $0.30-0.49$ & 0.38 & 0.21 & 147244 & \multirow{6}{*}{0.57} \\
\hline $0.50 \cdot 0.99$ & 0.70 & 0.30 & 38679 & \\
\hline $1.00-1.99$ & 1.41 & 0.30 & 7460 & \\
\hline $2.00-4.99$ & 3.16 & 0.40 & 4849 & \\
\hline $5.00-9.99$ & 7.07 & 0.30 & 297 & \\
\hline$>10.00$ & 14.14 & 0.30 & 195 & \\
\hline \multicolumn{5}{|l|}{ Sample \#9 } \\
\hline \begin{tabular}{|l} 
Bin \\
Range \\
$(u m)$
\end{tabular} & \begin{tabular}{|l} 
Bin Mean \\
Diameter \\
$(\omega \mathrm{m})$
\end{tabular} & $\begin{array}{l}\text { Diff. of the } \\
\text { log of the } \\
\text { Size }\end{array}$ & Counts & $\begin{array}{l}\text { Weighted } \\
\text { Mean } \\
\text { Diameter }\end{array}$ \\
\hline $0.30-0.49$ & 0.38 & 0.21 & 147006 & \multirow{6}{*}{0.61} \\
\hline $0.50-0.99$ & 0.70 & 0.30 & 39916 & \\
\hline $1.00-1.99$ & 1.41 & 0.30 & 7816 & \\
\hline $2.00-4.99$ & 3.16 & 0.40 & 5613 & \\
\hline $5.00-9.99$ & 7.07 & 0.30 & 379 & \\
\hline$>10.00$ & 14.14 & 0.30 & 507 & \\
\hline
\end{tabular}

Sample \#2

\begin{tabular}{|c|c|c|c|c|}
\hline $\begin{array}{l}\text { Bin } \\
\text { Range } \\
\text { (um) }\end{array}$ & $\begin{array}{l}\text { Bin Mean } \\
\text { Diameter } \\
(\text { (rm) }\end{array}$ & $\begin{array}{l}\text { Diff. of the } \\
\log \text { of the } \\
\text { Size }\end{array}$ & Counts & $\begin{array}{l}\text { Weighted } \\
\text { Mean } \\
\text { Diameter }\end{array}$ \\
\hline $0.30-0.49$ & .38 & 0.21 & 142433 & \multirow{6}{*}{0.61} \\
\hline $0.50-0.99$ & .70 & 0.30 & 39277 & \\
\hline $1.00-1.99$ & 1.41 & 0.30 & 8177 & \\
\hline $2.00-4.99$ & 3.16 & 0.40 & 5854 & \\
\hline $5.00-9.99$ & 7.07 & 0.30 & 410 & \\
\hline$>10.00$ & 14.14 & 0.30 & 433 & \\
\hline
\end{tabular}

\section{Sample \#4}

\begin{tabular}{|c|c|c|c|c|}
\hline $\begin{array}{l}\text { Bin } \\
\text { Range } \\
(u m)\end{array}$ & $\begin{array}{l}\text { Bin Mean } \\
\text { Diameter } \\
(\text { (um) }\end{array}$ & $\begin{array}{l}\text { Diff. of the } \\
\log \text { of the } \\
\text { Size }\end{array}$ & Counts & $\begin{array}{l}\text { Weighted } \\
\text { Mean } \\
\text { Diameter }\end{array}$ \\
\hline $0.30-0.49$ & .38 & 0.21 & 143855 & \multirow{6}{*}{0.60} \\
\hline $0.50 \cdot 0.99$ & .70 & 0.30 & 39164 & \\
\hline $1.00-1.99$ & 1.41 & 0.30 & 7832 & \\
\hline $2.00-4.99$ & 3.16 & 0.40 & 5399 & \\
\hline $5.00-9.99$ & 7.07 & 0.30 & 370 & \\
\hline$>10.00$ & 14.14 & 0.30 & 386 & \\
\hline
\end{tabular}

Sample \#6

\begin{tabular}{|c|c|c|c|c|}
\hline $\begin{array}{l}\text { Bin } \\
\text { Range } \\
(\mu \mathrm{m})\end{array}$ & $\begin{array}{l}\text { Bin Mean } \\
\text { Diameter } \\
(\text { (um) }\end{array}$ & $\begin{array}{l}\text { Diff. of the } \\
\text { log of the } \\
\text { Size }\end{array}$ & Counts & $\begin{array}{l}\text { Woighted } \\
\text { Mean } \\
\text { Diameter }\end{array}$ \\
\hline $0.30-0.49$ & .38 & 0.21 & 145637 & \multirow{6}{*}{0.58} \\
\hline $0.50-0.99$ & .70 & 0.30 & 39189 & \\
\hline $1.00-1.99$ & 1.41 & 0.30 & 7606 & \\
\hline $2.00-4.99$ & 3.16 & 0.40 & 5096 & \\
\hline $5.00-9.99$ & 7.07 & 0.30 & 314 & \\
\hline$>10.00$ & 14.14 & 0.30 & 231 & \\
\hline
\end{tabular}

Sample \#8

\begin{tabular}{|c|c|c|c|c|}
\hline \begin{tabular}{|l} 
Bin \\
Range \\
(um)
\end{tabular} & $\begin{array}{l}\text { Bin Mean } \\
\text { Diameter } \\
\text { (crm) }\end{array}$ & $\begin{array}{l}\text { Diff. of the } \\
\log \text { of the } \\
\text { Size }\end{array}$ & Counts & $\begin{array}{l}\text { Weighted } \\
\text { Mean } \\
\text { Diameter }\end{array}$ \\
\hline $0.30-0.49$ & .38 & 0.21 & 147346 & \multirow{6}{*}{0.58} \\
\hline $0.50-0.99$ & .70 & 0.30 & 39064 & \\
\hline $0.00-1.99$ & 1.41 & 0.30 & 7578 & \\
\hline $2.00-4.99$ & 3.16 & 0.40 & 5075 & \\
\hline $5.00-9.99$ & 7.07 & 0.30 & 338 & \\
\hline$>10.00$ & 14.14 & 0.30 & 255 & \\
\hline
\end{tabular}

\begin{tabular}{|c|c|c|c|c|}
\hline \multicolumn{5}{|c|}{ Sample \#10 } \\
\hline $\begin{array}{l}\text { Bin } \\
\text { Range } \\
\text { (um) }\end{array}$ & \begin{tabular}{|l} 
Bin Mean \\
Diameter \\
$(\mu \mathrm{m})$
\end{tabular} & $\begin{array}{l}\text { Diff. of the } \\
\log \text { of the } \\
\text { Size }\end{array}$ & Counts & $\begin{array}{l}\text { Weighted } \\
\text { Mean } \\
\text { Diameter }\end{array}$ \\
\hline $0.30-0.49$ & .38 & 0.21 & 149699 & \multirow{6}{*}{0.58} \\
\hline $0.50-0.99$ & .70 & 0.30 & 40089 & \\
\hline $1.00-1.99$ & 1.41 & 0.30 & 7709 & \\
\hline $2.00-4.99$ & 3.16 & 0.40 & 5085 & \\
\hline $5.00-9.99$ & 7.07 & 0.30 & 323 & \\
\hline$>10.00$ & 14.14 & 0.30 & 258 & \\
\hline
\end{tabular}




\section{Aerosol Particle Measurement Record}

Description of sample location 3706 Building, Room 102, Comparison Test, Sampler \#2, Date 7/22/94, Time sample started 13:26:56, Temperature 81.6 F, Humidity 39.3\%, Dew Point 55.6 F, Average Wind Velocity (if applicable) $.08 \mathrm{~m} / \mathrm{s}$, \# of physical barriers between sample area and outside (if applicable). $\underline{3}$,

Sample \#1

\begin{tabular}{|c|c|c|c|c|}
\hline $\begin{array}{l}\text { Bin } \\
\text { Range } \\
\text { (um) }\end{array}$ & $\begin{array}{l}\text { Bin Mean } \\
\text { Diameter } \\
(\text { (um) }\end{array}$ & $\begin{array}{l}\text { Diff. of the } \\
\text { log of the } \\
\text { Size }\end{array}$ & Counts & $\begin{array}{l}\text { Weighted } \\
\text { Mean } \\
\text { Diameter }\end{array}$ \\
\hline $0.30-0.49$ & 0.38 & 0.21 & 121368 & \multirow{6}{*}{0.65} \\
\hline $0.50-0,99$ & 0.70 & 0.30 & 69701 & \\
\hline $1.00-1.99$ & 1.41 & 0.30 & 6480 & \\
\hline $2.00-4.99$ & 3.16 & 0.40 & 5654 & \\
\hline $5.00 \cdot 9.99$ & 7.07 & 0.30 & 566 & \\
\hline$>10.00$ & 14.14 & 0.30 & 430 & \\
\hline
\end{tabular}

\section{Sample \#3}

\begin{tabular}{|c|c|c|c|c|}
\hline \begin{tabular}{|l} 
Bin \\
Range \\
$(\mu \mathrm{m})$
\end{tabular} & \begin{tabular}{|l|} 
Bin Mean \\
Diameter \\
$(\mu \dot{m})$
\end{tabular} & $\begin{array}{l}\text { Diff. of the } \\
\log \text { of the } \\
\text { Size }\end{array}$ & Counts & $\begin{array}{l}\text { Weighted } \\
\text { Mean } \\
\text { Diameter }\end{array}$ \\
\hline $0.30-0.49$ & 0.38 & 0.21 & 122800 & \multirow{6}{*}{0.63} \\
\hline $0.50-0.99$ & 0.70 & 0.30 & 67959 & \\
\hline $1.00-1.99$ & 1.41 & 0.30 & 5789 & \\
\hline $2.00-4.99$ & 3.16 & 0.40 & 5188 & \\
\hline $5.00-9.99$ & 7.07 & 0.30 & 475 & \\
\hline$>10.00$ & 14.14 & 0.30 & 343 & \\
\hline
\end{tabular}

Sample \#5

\begin{tabular}{|c|c|c|c|c|}
\hline $\begin{array}{l}\text { Bin } \\
\text { Range } \\
(\mu \mathrm{m})\end{array}$ & \begin{tabular}{|l}
$\begin{array}{l}\text { Bin Mean } \\
\text { Diameter } \\
(\mu \mathrm{m})\end{array}$ \\
\end{tabular} & $\begin{array}{l}\text { Diff. of the } \\
\text { log of the } \\
\text { Size }\end{array}$ & Counts & $\begin{array}{l}\text { Weighted } \\
\text { Mean } \\
\text { Diameter }\end{array}$ \\
\hline $0.30 \cdot 0.49$ & 0.38 & 0.21 & 123425 & \multirow{6}{*}{0.60} \\
\hline $0.50-0.99$ & 0.70 & 0.30 & 67193 & \\
\hline $1.00-1.99$ & 1.41 & 0.30 & 5330 & \\
\hline $2.00-4.99$ & 3.16 & 0.40 & 4606 & \\
\hline $5.00-9.99$ & 7.07 & 0.30 & 341 & \\
\hline$>10.00$ & 14.14 & 0.30 & 186 & \\
\hline
\end{tabular}

Sample \#7

\begin{tabular}{||c|c|c|r|r||}
\hline \begin{tabular}{||c|c|r||} 
Bin \\
Range \\
$(\mu \mathrm{m})$
\end{tabular} & $\begin{array}{l}\text { Bin Mean } \\
\text { Diameter } \\
(\mu \mathrm{m})\end{array}$ & $\begin{array}{l}\text { Diff. of the } \\
\text { log of the } \\
\text { Size }\end{array}$ & Counts & $\begin{array}{l}\text { Weighted } \\
\text { Mean } \\
\text { Diameter }\end{array}$ \\
\hline $0.30 \cdot 0.49$ & 0.38 & 0.21 & 126492 & \\
\hline $0.50-0.99$ & 0.70 & 0.30 & 68613 & \multirow{2}{*}{0.57} \\
\hline $1.00-1.99$ & 1.41 & 0.30 & 5123 & \\
\hline $2.00-4.99$ & 3.16 & 0.40 & 4218 & \\
\hline $5.00-9.99$ & 7.07 & 0.30 & 316 & \\
\hline$>10.00$ & 14.14 & 0.30 & 130 & \\
\hline
\end{tabular}

\begin{tabular}{|c|c|c|c|c|}
\hline $\begin{array}{l}\text { Bin } \\
\text { Range } \\
(\mu \mathrm{m})\end{array}$ & $\begin{array}{l}\text { Bin Mean } \\
\text { Diameter } \\
(u m)\end{array}$ & $\begin{array}{l}\text { Diff. of the } \\
\text { log of the } \\
\text { Size }\end{array}$ & Counts & $\begin{array}{l}\text { Weighted } \\
\text { Mean } \\
\text { Diameter }\end{array}$ \\
\hline $0.30-0.49$ & 0.38 & 0.21 & 125180 & \multirow{6}{*}{0.62} \\
\hline $0.50-0.99$ & 0.70 & 0.30 & 69466 & \\
\hline $1.00-1.99$ & 1.41 & 0.30 & 5334 & \\
\hline $2.00-4.99$ & 3.16 & 0.40 & 4749 & \\
\hline $5.00-9.99$ & 7.07 & 0.30 & 409 & \\
\hline$>10.00$ & 74.14 & 0.30 & 346 & \\
\hline
\end{tabular}

Sample \#2

\begin{tabular}{|c|c|c|c|c|}
\hline \begin{tabular}{|l|} 
Bin \\
Range \\
$(\mu \mathrm{m})$
\end{tabular} & \begin{tabular}{|l|} 
Bin Mean \\
Diameter \\
$(u m)$
\end{tabular} & $\begin{array}{l}\text { Diff. of the } \\
\log \text { of the } \\
\text { Size }\end{array}$ & Counts & $\begin{array}{l}\text { Weighted } \\
\text { Mean } \\
\text { Diameter }\end{array}$ \\
\hline $0.30-0.49$ & .38 & 0.21 & 126968 & \multirow{6}{*}{0.62} \\
\hline $0.50-0.99$ & .70 & 0.30 & 69728 & \\
\hline $1.00-1.99$ & 1.41 & 0.30 & 5802 & \\
\hline $2.00-4.99$ & 3.16 & 0.40 & 5243 & \\
\hline $5.00-9.99$ & 7.07 & 0.30 & 441 & \\
\hline$>10.00$ & 14.14 & 0.30 & 310 & \\
\hline
\end{tabular}

\begin{tabular}{|c|c|c|c|c|}
\hline \multicolumn{5}{|l|}{ Sample \#4 } \\
\hline $\begin{array}{l}\text { Bin } \\
\text { Range } \\
\text { ( } \mathrm{m})\end{array}$ & $\begin{array}{l}\text { Bin Mean } \\
\text { Diameter } \\
(\mu \mathrm{m})\end{array}$ & $\begin{array}{l}\text { Diff. of the } \\
\text { log of the } \\
\text { Size }\end{array}$ & Counts & $\begin{array}{l}\text { Weighted } \\
\text { Mean } \\
\text { Diameter }\end{array}$ \\
\hline $0.30-0.49$ & .38 & 0.21 & 123602 & \multirow{6}{*}{0.61} \\
\hline $0.50-0.99$ & .70 & 0.30 & 67886 & \\
\hline $1.00-1.99$ & 1.41 & 0.30 & 5546 & \\
\hline $2.00-4.99$ & 3.16 & 0.40 & 4805 & \\
\hline $5.00-9.99$ & 7.07 & 0.30 & 399 & \\
\hline$>10.00$ & 14.14 & 0.30 & 267 & \\
\hline
\end{tabular}

Sample \#6

\begin{tabular}{|c|c|c|c|c|}
\hline $\begin{array}{l}\text { Bin } \\
\text { Range } \\
(\mu \mathrm{m})\end{array}$ & $\begin{array}{l}\text { Bin Mean } \\
\text { Diameter } \\
(\mu m)\end{array}$ & $\begin{array}{l}\text { Diff. of the } \\
\log \text { of the } \\
\text { Size }\end{array}$ & Counts & $\begin{array}{l}\text { Weighted } \\
\text { Mean } \\
\text { Diameter }\end{array}$ \\
\hline $0.30-0.49$ & .38 & 0.21 & 125822 & \multirow{6}{*}{0.60} \\
\hline $0.50-0.99$ & .70 & 0.30 & 68700 & \\
\hline $1.00-1.99$ & 1.41 & 0.30 & 5423 & \\
\hline $2.00-4.99$ & 3.16 & $\overline{0.40}$ & 4454 & \\
\hline $5.00-9.99$ & 7.07 & 0.30 & 329 & \\
\hline$>10.00$ & 14.14 & 0.30 & 161 & \\
\hline
\end{tabular}

\begin{tabular}{|c|c|c|c|c|}
\hline \multicolumn{5}{|c|}{ Sample $\# 8$} \\
\hline \begin{tabular}{|l} 
Bin \\
Range \\
$(\mathrm{um})$
\end{tabular} & \begin{tabular}{|l} 
Bin Mean \\
Diameter \\
$(\mathrm{um})$
\end{tabular} & $\begin{array}{l}\text { Diff. of the } \\
\text { log of the } \\
\text { Size }\end{array}$ & Counts & $\begin{array}{l}\text { Weighted } \\
\text { Mean } \\
\text { Diameter }\end{array}$ \\
\hline $0.30-0.49$ & .38 & 0.21 & 127441 & \multirow{6}{*}{0.58} \\
\hline $0.50-0.99$ & .70 & 0.30 & 69252 & \\
\hline $0.00-1.99$ & 1.41 & 0.30 & 5147 & \\
\hline $2.00-4.99$ & 3.16 & 0.40 & 4273 & \\
\hline $5.00-9.99$ & 7.07 & 0.30 & 313 & \\
\hline$>10.00$ & 14.14 & 0.30 & 159 & \\
\hline
\end{tabular}

\begin{tabular}{|c|c|c|c|c|}
\hline \multicolumn{5}{|c|}{ Sample \#10 } \\
\hline \begin{tabular}{|l|} 
Bin \\
Range \\
$(\mu \mathrm{m})$
\end{tabular} & \begin{tabular}{|l} 
Bin Mean \\
Diameter \\
$(\mu \mathrm{m})$
\end{tabular} & $\begin{array}{l}\text { Diff. of the } \\
\text { log of the } \\
\text { Size }\end{array}$ & Counts & $\begin{array}{l}\text { Weighted } \\
\text { Mean } \\
\text { Diameter }\end{array}$ \\
\hline $0.30-0.49$ & .38 & 0.21 & 126891 & \multirow{6}{*}{0.60} \\
\hline $0.50-0.99$ & .70 & 0.30 & 69724 & \\
\hline $1.00-1.99$ & 1.41 & 0.30 & 5226 & \\
\hline $2.00-4.99$ & 3.16 & 0.40 & 4322 & \\
\hline $5.00-9.99$ & 7.07 & 0.30 & 333 & \\
\hline$>10.00$ & 14.14 & 0.30 & 206 & \\
\hline
\end{tabular}


Data for Table 3. Mean Optical Diameter of Ambient Air, Building 3706A

$2 \mathrm{~cm} \cdot(0.02$ meters $)$

0.5 meters

1.0 meters

1.5 meters

2.0 meters 


\section{Aerosol Particle Measurement Record}

Description of sample location 3706A Building, Ambient Air Test, at $2 \mathrm{~cm}$, Date 8/9/94 Time sample started_11:03:47, Temperature_76.3 F, Humidity 40.1\%, Dew Point 51.1 F , Average Wind Velocity (if applicable) .00 m/s, \# of physical barriers between sample area and outside (if applicable) 1

\begin{tabular}{|c|c|c|c|c|}
\hline $\begin{array}{l}\text { Bin } \\
\text { Range } \\
(\mu \mathrm{m})\end{array}$ & \begin{tabular}{|l|} 
Bin Mean \\
Diameter \\
$(u \mathrm{~m})$
\end{tabular} & $\begin{array}{l}\text { Diff. of the } \\
\log \text { of the } \\
\text { Size }\end{array}$ & Counts & \begin{tabular}{|l} 
Weighted \\
Mean \\
Diameter
\end{tabular} \\
\hline $0.30 \cdot 0.49$ & 0.38 & 0.21 & 135152 & \multirow{6}{*}{0.60} \\
\hline $0.50-0.99$ & 0.70 & 0.30 & 39797 & \\
\hline $1.00-1.99$ & 1.41 & 0.30 & 8036 & \\
\hline $2.00-4.99$ & 3.16 & 0.40 & 5650 & \\
\hline $5.00-9.99$ & 7.07 & 0.30 & 460 & \\
\hline$>10.00$ & 14.14 & 0.30 & 122 & \\
\hline \multicolumn{5}{|l|}{ Sample \#3 } \\
\hline $\begin{array}{l}\text { Bin } \\
\text { Range } \\
(\mu \mathrm{m})\end{array}$ & \begin{tabular}{|l} 
Bin Mean \\
Diameter \\
$(u m)$
\end{tabular} & $\begin{array}{l}\text { Diff. of the } \\
\text { log of the } \\
\text { Size }\end{array}$ & Counts & \begin{tabular}{|l} 
Weighted \\
Mean \\
Diameter
\end{tabular} \\
\hline $0.30-0.49$ & 0.38 & 0.21 & 132461 & \multirow{6}{*}{0.60} \\
\hline $0.50-0.99$ & 0.70 & 0.30 & 39053 & \\
\hline $1.00-1.99$ & 1.41 & 0.30 & 7817 & \\
\hline $2.00 \cdot 4.99$ & 3.16 & 0.40 & 5445 & \\
\hline $5.00-9.99$ & 7.07 & 0.30 & 454 & \\
\hline$>10.00$ & 14.14 & 0.30 & 94 & \\
\hline \multicolumn{5}{|l|}{ Sample \#5 } \\
\hline \begin{tabular}{|l} 
Bin \\
Range \\
$(\mu \mathrm{m})$
\end{tabular} & $\begin{array}{l}\text { Bin Mean } \\
\text { Diameter } \\
(\mu \mathrm{m})\end{array}$ & $\begin{array}{l}\text { Diff. of the } \\
\text { log of the } \\
\text { Size }\end{array}$ & Counts & \begin{tabular}{|l|} 
Weighted \\
Mean \\
Diameter
\end{tabular} \\
\hline $0.30-0.49$ & 0.38 & 0.21 & 129545 & \multirow{6}{*}{0.60} \\
\hline $0.50-0.99$ & 0.70 & 0.30 & 37777 & \\
\hline $1.00 \cdot 1.99$ & 1.41 & 0.30 & 7630 & \\
\hline $2.00 \cdot 4.99$ & 3.16 & 0.40 & 5334 & \\
\hline $5.00-9.99$ & 7.07 & 0.30 & 464 & \\
\hline$>10.00$ & 14.14 & 0.30 & 120 & \\
\hline \multicolumn{5}{|l|}{ Sample \#7 } \\
\hline \begin{tabular}{|l} 
Bin \\
Range \\
$(u \mathrm{~m})$
\end{tabular} & \begin{tabular}{|l|} 
Bin Mean \\
Diameter \\
$(\mu \mathrm{m})$
\end{tabular} & \begin{tabular}{|l} 
Diff. of the \\
log of the \\
Size
\end{tabular} & Counts & $\begin{array}{l}\text { Weighted } \\
\text { Mean } \\
\text { Diameter }\end{array}$ \\
\hline $0.30-0.49$ & 0.38 & 0.21 & 126421 & \multirow{6}{*}{0.59} \\
\hline $0.50-0.99$ & 0.70 & 0.30 & 36925 & \\
\hline $1.00-1.99$ & 1.41 & 0.30 & 7322 & \\
\hline $2.00 \cdot 4.99$ & 3.16 & 0.40 & 4980 & \\
\hline $5.00-9.99$ & 7.07 & 0.30 & 399 & \\
\hline$>10.00$ & 14.14 & 0.30 & 126 & \\
\hline \multicolumn{5}{|l|}{ Sample \#9 } \\
\hline \begin{tabular}{|l} 
Bin \\
Range \\
$(\mu \mathrm{m})$
\end{tabular} & \begin{tabular}{|l} 
Bin Mean \\
Diameter \\
$(\mu m)$
\end{tabular} & $\begin{array}{l}\text { Diff. of the } \\
\log \text { of the } \\
\text { Size }\end{array}$ & Counts & $\begin{array}{l}\text { Weighted } \\
\text { Mean } \\
\text { Diameter }\end{array}$ \\
\hline $0.30-0.49$ & 0.38 & 0.21 & 125088 & \multirow{6}{*}{0.59} \\
\hline $0.50-0.99$ & 0.70 & 0.30 & 36183 & \\
\hline $1.00-1.99$ & 1.41 & 0.30 & 7102 & \\
\hline $2.00-4.99$ & 3.16 & 0.40 & 4868 & \\
\hline $5.00-9.99$ & 7.07 & 0.30 & 355 & \\
\hline$>10.00$ & 14.14 & 0.30 & 86 & \\
\hline
\end{tabular}

\begin{tabular}{|c|c|c|c|c|}
\hline $\begin{array}{l}\text { Bin } \\
\text { Range } \\
\text { (um) }\end{array}$ & $\begin{array}{l}\text { Bin Mean } \\
\text { Diameter } \\
(\mu \mathrm{m})\end{array}$ & $\begin{array}{l}\text { Diff. of the } \\
\log \text { of the } \\
\text { Size }\end{array}$ & Counts & $\begin{array}{l}\text { Weighted } \\
\text { Mean } \\
\text { Diameter }\end{array}$ \\
\hline $0.30-0.49$ & .38 & 0.21 & 135059 & \multirow{6}{*}{0.59} \\
\hline $0.50-0.99$ & .70 & 0.30 & 39800 & \\
\hline $1.00-1.99$ & 1.41 & 0.30 & 7970 & \\
\hline $2.00-4.99$ & 3.16 & 0.40 & 5459 & \\
\hline $5.00-9.99$ & 7.07 & 0.30 & 429 & \\
\hline$>10.00$ & 14.14 & 0.30 & $\overrightarrow{104}$ & \\
\hline
\end{tabular}

\begin{tabular}{|c|c|c|c|c|}
\hline \multicolumn{5}{|l|}{ Sample \#4 } \\
\hline $\begin{array}{l}\text { Bin } \\
\text { Range } \\
(u \mathrm{~m})\end{array}$ & $\begin{array}{l}\text { Bin Mean } \\
\text { Diameter } \\
(\mu \mathrm{m})\end{array}$ & $\begin{array}{l}\text { Diff. of the } \\
\text { log of the } \\
\text { Size }\end{array}$ & Counts & $\begin{array}{l}\text { Weighted } \\
\text { Mean } \\
\text { Diameter }\end{array}$ \\
\hline $0.30-0.49$ & .38 & 0.21 & 129590 & \multirow{6}{*}{0.60} \\
\hline $0.50-0.99$ & .70 & 0.30 & 38751 & \\
\hline $1.00-1.99$ & 1.41 & 0.30 & 8028 & \\
\hline $2.00-4.99$ & 3.16 & 0.40 & 5530 & \\
\hline $5.00-9.99$ & 7.07 & 0.30 & 437 & \\
\hline$>10.00$ & 14.14 & 0.30 & 115 & \\
\hline
\end{tabular}

\begin{tabular}{|c|c|c|c|c|}
\hline \multicolumn{5}{|l|}{ Sample \#6 } \\
\hline $\begin{array}{l}\text { Bin } \\
\text { Range } \\
(\mu \mathrm{m})\end{array}$ & $\begin{array}{l}\text { Bin Mean } \\
\text { Diameter } \\
(\mu \mathrm{m})\end{array}$ & $\begin{array}{l}\text { Diff. of the } \\
\text { log of the } \\
\text { Size }\end{array}$ & Counts & $\begin{array}{l}\text { Weighted } \\
\text { Mean } \\
\text { Diameter }\end{array}$ \\
\hline $0.30-0.49$ & .38 & 0.21 & 127052 & \multirow{6}{*}{0.60} \\
\hline $0.50-0.99$ & .70 & 0.30 & 37155 & \\
\hline $1.00-1.99$ & 1.41 & 0.30 & 7413 & \\
\hline $2.00-4.99$ & 3.16 & 0.40 & 5168 & \\
\hline $5.00-9.99$ & 7.07 & 0.30 & 450 & \\
\hline$>10.00$ & 14.14 & 0.30 & 127 & \\
\hline
\end{tabular}

\begin{tabular}{|c|c|c|c|c|}
\hline \multicolumn{5}{|l|}{ Sample \#8 } \\
\hline $\begin{array}{l}\text { Bin } \\
\text { Range } \\
(\mu \mathrm{m})\end{array}$ & $\begin{array}{l}\text { Bin Mean } \\
\text { Diameter } \\
(\mu \mathrm{m})\end{array}$ & $\begin{array}{l}\text { Diff. of the } \\
\text { log of the } \\
\text { Size }\end{array}$ & Counts & $\begin{array}{l}\text { Weighted } \\
\text { Mean } \\
\text { Diameter }\end{array}$ \\
\hline $0.30-0.49$ & .38 & 0.21 & 125403 & \multirow{6}{*}{0.60} \\
\hline $0.50-0.99$ & .70 & 0.30 & 36558 & \\
\hline $0.00-1.99$ & 1.41 & 0.30 & 7387 & \\
\hline $2.00-4.99$ & 3.16 & 0.40 & 5160 & \\
\hline $5.00-9.99$ & 7.07 & 0.30 & 422 & \\
\hline$>10.00$ & 14.14 & 0.30 & $\overline{109}$ & \\
\hline
\end{tabular}

Sample $\# 10$

\begin{tabular}{|c|c|c|c|c|}
\hline $\begin{array}{l}\text { Bin } \\
\text { Range } \\
\text { (um) }\end{array}$ & \begin{tabular}{|l|} 
Bin Mean \\
Diameter \\
$(\mathrm{um})$
\end{tabular} & $\begin{array}{l}\text { Diff. of the } \\
\text { log of the } \\
\text { Size }\end{array}$ & Counts & $\begin{array}{l}\text { Weighted } \\
\text { Mean } \\
\text { Diameter }\end{array}$ \\
\hline $0.30-0.49$ & .38 & 0.21 & 123829 & \multirow{6}{*}{0.59} \\
\hline $0.50-0.99$ & .70 & 0.30 & 35454 & \\
\hline $1.00-1.99$ & 1.41 & 0.30 & 6785 & \\
\hline $2.00-4.99$ & 3.16 & 0.40 & $\overline{4774}$ & \\
\hline $5.00-9.99$ & 7.07 & 0.30 & 390 & \\
\hline$>10.00$ & 14.14 & 0.30 & 111 & \\
\hline
\end{tabular}




\section{Aerosol Particle Measurement Record}

Description of sample location 3706A Building, Ambient Air Test, at $0.5 \mathrm{~m}$, Date 8/9/94, Time sample started 10:20:48, Temperature 73.9 F, Humidity 43.0\%, Dew Point 50.0 F - Average Wind Velocity (if applicable) . $.00 \mathrm{~m} / \mathrm{s}$, \# of physical barriers between sample area and outside (if applicable) 1

\begin{tabular}{|c|c|c|c|c|}
\hline $\begin{array}{l}\text { Bin } \\
\text { Range } \\
(\mu \mathrm{m})\end{array}$ & \begin{tabular}{|l} 
Bin Mean \\
Diameter \\
$($ (um)
\end{tabular} & $\begin{array}{l}\text { Diff. of the } \\
\text { log of the } \\
\text { Size }\end{array}$ & Counts & $\begin{array}{l}\text { Weighted } \\
\text { Mean } \\
\text { Diameter }\end{array}$ \\
\hline $0.30-0.49$ & 0.38 & 0.21 & 165750 & \multirow{6}{*}{0.56} \\
\hline $0.50-0.99$ & 0.70 & 0.30 & 49311 & \\
\hline $1.00-1.99$ & 1.41 & 0.30 & 8485 & \\
\hline $2.00-4.99$ & 3.16 & 0.40 & 4702 & \\
\hline $5.00-9.99$ & 7.07 & 0.30 & 339 & \\
\hline$>10.00$ & 14.14 & 0.30 & 110 & \\
\hline \multicolumn{5}{|l|}{ Sample \#3 } \\
\hline $\begin{array}{l}\text { Bin } \\
\text { Range } \\
(\mu \mathrm{m})\end{array}$ & \begin{tabular}{|l} 
Bin Mean \\
Diameter \\
(um)
\end{tabular} & $\begin{array}{l}\text { Diff. of the } \\
\log \text { of the } \\
\text { Size }\end{array}$ & Counts & $\begin{array}{l}\text { Weighted } \\
\text { Mean } \\
\text { Diameter }\end{array}$ \\
\hline $0.30-0.49$ & 0.38 & 0.21 & 163224 & \multirow{6}{*}{0.57} \\
\hline $0.50-0.99$ & 0.70 & 0.30 & 48396 & \\
\hline $1.00-1.99$ & 1.41 & 0.30 & 8577 & \\
\hline $2.00-4.99$ & 3.16 & 0.40 & 5137 & \\
\hline $5.00-9.99$ & 7.07 & 0.30 & 423 & \\
\hline$>10.00$ & 14.14 & 0.30 & 118 & \\
\hline \multicolumn{5}{|l|}{ Sample \#5 } \\
\hline \begin{tabular}{|l|} 
Bin \\
Range \\
$(\mu \mathrm{m})$ \\
\end{tabular} & \begin{tabular}{|l} 
Bin Mean \\
Diameter \\
( $/ \mathrm{rm})$
\end{tabular} & \begin{tabular}{|l|} 
Diff. of the \\
log of the \\
Size
\end{tabular} & Counts & $\begin{array}{l}\text { Weighted } \\
\text { Mean } \\
\text { Diameter }\end{array}$ \\
\hline $0.30 \cdot 0.49$ & 0.38 & 0.21 & 162409 & \multirow{6}{*}{0.57} \\
\hline $0.50 \cdot 0.99$ & 0.70 & 0.30 & 48067 & \\
\hline $1.00 \cdot 1.99$ & 1.41 & 0.30 & 8851 & \\
\hline $2.00-4.99$ & 3.16 & 0.40 & 5436 & \\
\hline $5.00-9.99$ & 7.07 & 0.30 & 364 & \\
\hline \begin{tabular}{|l} 
\\
\end{tabular} & 14.14 & 0.30 & 121 & \\
\hline \multicolumn{5}{|l|}{ Sample \#7 } \\
\hline \begin{tabular}{|l} 
Bin \\
Range \\
$(y \mathrm{~m})$
\end{tabular} & $\begin{array}{l}\text { Bin Mean } \\
\text { Diameter } \\
(\omega \mathrm{m})\end{array}$ & \begin{tabular}{|l|} 
Diff. of the \\
log of the \\
Size
\end{tabular} & Counts & $\begin{array}{l}\text { Weighted } \\
\text { Mean } \\
\text { Diameter }\end{array}$ \\
\hline $0.30-0.49$ & 0.38 & 0.21 & 160925 & \multirow{6}{*}{0.57} \\
\hline $0.50-0.99$ & 0.70 & 0.30 & 46928 & \\
\hline $1.00-1.99$ & 1.41 & 0.30 & 8528 & \\
\hline $2.00 \cdot 4.99$ & 3.16 & 0.40 & 5135 & \\
\hline $5.00-9.99$ & 7.07 & 0.30 & 415 & \\
\hline$>10.00$ & 14.14 & 0.30 & 115 & \\
\hline \multicolumn{5}{|l|}{ Sample \#9 } \\
\hline \begin{tabular}{|l} 
Bin \\
Range \\
$(\mu \mathrm{m})$
\end{tabular} & \begin{tabular}{|l} 
Bin Mean \\
Diameter \\
$(u m)$
\end{tabular} & \begin{tabular}{|l|} 
Diff. of the \\
log of the \\
Size
\end{tabular} & Counts & $\begin{array}{l}\text { Weighted } \\
\text { Mean } \\
\text { Diameter }\end{array}$ \\
\hline $0.30 \cdot 0.49$ & 0.38 & 0.21 & 157541 & \multirow{6}{*}{0.57} \\
\hline $0.50-0.99$ & 0.70 & 0.30 & 45947 & \\
\hline $1.00-1.99$ & 1.41 & 0.30 & 8367 & \\
\hline $2.00 \cdot 4.99$ & 3.16 & 0.40 & 5104 & \\
\hline $5.00-9.99$ & 7.07 & 0.30 & 415 & \\
\hline$>10.00$ & 14.14 & 0.30 & 106 & \\
\hline
\end{tabular}

\begin{tabular}{|c|c|c|c|c|}
\hline $\begin{array}{l}\text { Bin } \\
\text { Range } \\
(\mathrm{um})\end{array}$ & \begin{tabular}{|l|} 
Bin Mean \\
Diameter \\
$(u m)$
\end{tabular} & $\begin{array}{l}\text { Diff. of the } \\
\log \text { of the } \\
\text { Size }\end{array}$ & Counts & $\begin{array}{l}\text { Weighted } \\
\text { Mean } \\
\text { Diamoter }\end{array}$ \\
\hline $0.30-0.49$ & .38 & 0.21 & 164140 & \multirow{6}{*}{0.56} \\
\hline $0.50-0.99$ & .70 & 0.30 & 48379 & \\
\hline $1.00-1.99$ & 1.41 & 0.30 & 8286 & \\
\hline $2.00-4.99$ & 3.16 & 0.40 & 4797 & \\
\hline $5.00-9.99$ & 7.07 & 0.30 & 396 & \\
\hline$>10.00$ & 14.14 & 0.30 & 110 & \\
\hline
\end{tabular}

\begin{tabular}{|c|c|c|c|c|}
\hline \multicolumn{5}{|l|}{ Sample \#4 } \\
\hline $\begin{array}{l}\text { Bin } \\
\text { Range } \\
(u m)\end{array}$ & \begin{tabular}{|l} 
Bin Mean \\
Diameter \\
$(u m)$
\end{tabular} & $\begin{array}{l}\text { Diff. of the } \\
\log \text { of the } \\
\text { Size }\end{array}$ & Counts & $\begin{array}{l}\text { Weighted } \\
\text { Mean } \\
\text { Diameter }\end{array}$ \\
\hline $0.30-0.49$ & .38 & 0.21 & 162114 & \multirow{6}{*}{0.58} \\
\hline $0.50-0.99$ & .70 & 0.30 & 48755 & \\
\hline $1.00-1.99$ & 1.41 & 0.30 & 8934 & \\
\hline $2.00-4.99$ & . 3.16 & 0.40 & 5775 & \\
\hline $5.00-9.99$ & 7.07 & 0.30 & 470 & \\
\hline$>10.00$ & 14.14 & 0.30 & 120 & \\
\hline
\end{tabular}

\begin{tabular}{|c|c|c|c|c|}
\hline \begin{tabular}{|l} 
Bin \\
Range \\
$(u m)$
\end{tabular} & \begin{tabular}{|l} 
Bin Mean \\
Diameter \\
$(\mu \mathrm{m})$
\end{tabular} & $\begin{array}{l}\text { Diff. of the } \\
\text { log of the } \\
\text { Size }\end{array}$ & Counts & $\begin{array}{l}\text { Weighted } \\
\text { Mean } \\
\text { Diameter }\end{array}$ \\
\hline $0.30-0.49$ & .38 & 0.21 & 161078 & \multirow{6}{*}{0.57} \\
\hline $0.50-0.99$ & .70 & 0.30 & 47935 & \\
\hline $1.00-1.99$ & 1.41 & 0.30 & 8528 & \\
\hline $2.00-4.99$ & 3.16 & 0.40 & 5194 & \\
\hline $5.00-9.99$ & 7.07 & 0.30 & 404 & \\
\hline$>10.00$ & $\overline{14.14}$ & 0.30 & 115 & \\
\hline
\end{tabular}

\begin{tabular}{|c|c|c|c|c|}
\hline \multicolumn{5}{|l|}{ Sample \#7 } \\
\hline $\begin{array}{l}\text { Bin } \\
\text { Range } \\
(\mu \mathrm{m})\end{array}$ & \begin{tabular}{|l} 
Bin Mean \\
Diameter \\
$(\mathrm{um})$
\end{tabular} & $\begin{array}{l}\text { Diff. of the } \\
\log \text { of the } \\
\text { Size }\end{array}$ & Counts & $\begin{array}{l}\text { Weighted } \\
\text { Mean } \\
\text { Diameter }\end{array}$ \\
\hline $0.30-0.49$ & .38 & 0.21 & 159261 & \multirow{6}{*}{0.57} \\
\hline $0.50-0.99$ & .70 & 0.30 & 46577 & \\
\hline $0.00-1.99$ & 1.41 & 0.30 & 8546 & \\
\hline $2.00-4.99$ & 3.16 & 0.40 & 5030 & \\
\hline $5.00-9.99$ & 7.07 & 0.30 & 388 & \\
\hline$>10.00$ & 14.14 & 0.30 & 93 & \\
\hline
\end{tabular}

\begin{tabular}{|c|c|c|c|c|}
\hline $\begin{array}{l}\text { Bin } \\
\text { Range } \\
\text { (um) }\end{array}$ & \begin{tabular}{|l|} 
Bin Mean \\
Diameter \\
$(\mu \mathrm{m})$
\end{tabular} & $\begin{array}{l}\text { Diff. of the } \\
\text { log of the } \\
\text { Size }\end{array}$ & Counts & $\begin{array}{l}\text { Weighted } \\
\text { Mean } \\
\text { Diameter }\end{array}$ \\
\hline $0.30-0.49$ & .38 & 0.21 & 155565 & \multirow{6}{*}{0.57} \\
\hline $0.50-0.99$ & .70 & 0.30 & 46190 & \\
\hline $1.00-1.99$ & 1.41 & 0.30 & 8129 & \\
\hline $2.00-4.99$ & 3.16 & 0.40 & 5130 & \\
\hline $5.00-9.99$ & 7.07 & 0.30 & 372 & \\
\hline$>10.00$ & 14.14 & 0.30 & 129 & \\
\hline
\end{tabular}




\section{Aerosol Particle Measurement Record}

Description of sample location 3706 A Building. Ambient Air Test, at $1.0 \mathrm{~m}$, Date $8 / 9 / 94$. Time sample started 9:46:48, Temperature 73.3 F, Humidity 43.0\%, Dew Point 50.0 F, Average Wind Velocity (if applicable) $.00 \mathrm{~m} / \mathrm{s}$, \# of physical barriers between sample area and outside (if applicable) 1

Sample \#1

\begin{tabular}{|c|c|c|c|c|}
\hline $\begin{array}{l}\text { Bin } \\
\text { Range } \\
\text { (um) }\end{array}$ & \begin{tabular}{|l} 
Bin Mean \\
Diameter \\
$(\mu \mathrm{m})$
\end{tabular} & $\begin{array}{l}\text { Diff. of the } \\
\text { log of the } \\
\text { Size }\end{array}$ & Counts & $\begin{array}{l}\text { Weighted } \\
\text { Mean } \\
\text { Diameter }\end{array}$ \\
\hline $0.30 \cdot 0.49$ & 0.38 & 0.21 & 132781 & \multirow{6}{*}{0.56} \\
\hline $0.50-0.99$ & 0.70 & 0.30 & 84393 & \\
\hline $1.00-1.99$ & 1.41 & 0.30 & 4402 & \\
\hline $2.00-4.99$ & 3.16 & 0.40 & 2723 & \\
\hline $5.00-9.99$ & 7.07 & 0.30 & 150 & \\
\hline$>10.00$ & 14.14 & 0.30 & 102 & \\
\hline \multicolumn{5}{|l|}{ Sample \#3 } \\
\hline \begin{tabular}{|l|} 
Bin \\
Range \\
$(\mu \mathrm{m})$ \\
\end{tabular} & \begin{tabular}{|l|}
$\begin{array}{l}\text { Bin Mean } \\
\text { Diameter } \\
(\mu \mathrm{m})\end{array}$ \\
\end{tabular} & $\begin{array}{l}\text { Diff. of the } \\
\text { log of the } \\
\text { Size }\end{array}$ & Counts & \begin{tabular}{|l} 
Weighted \\
Mean \\
Diameter
\end{tabular} \\
\hline $0.30-0.49$ & 0.38 & 0.21 & 134416 & \multirow{6}{*}{0.57} \\
\hline $0.50 \cdot 0.99$ & 0.70 & 0.30 & 85217 & \\
\hline $1.00-1.99$ & 1.41 & 0.30 & 4493 & \\
\hline $2.00-4.99$ & 3.16 & 0.40 & 2826 & \\
\hline $5.00-9.99$ & 7.07 & 0.30 & 154 & \\
\hline$>10.00$ & 14.14 & 0.30 & 123 & \\
\hline \multicolumn{5}{|l|}{ Sample \#5 } \\
\hline \begin{tabular}{|l|}
$\begin{array}{l}\text { Bin } \\
\text { Range } \\
(\mu \mathrm{m})\end{array}$ \\
\end{tabular} & $\begin{array}{l}\text { Bin Mean } \\
\text { Diameter } \\
(\mu \mathrm{m})\end{array}$ & $\begin{array}{l}\text { Diff. of the } \\
\text { log of the } \\
\text { Size }\end{array}$ & Counts & $\begin{array}{l}\text { Weighted } \\
\text { Mean } \\
\text { Diameter }\end{array}$ \\
\hline $0.30-0.49$ & 0.38 & 0.21 & 131170 & \multirow{6}{*}{0.56} \\
\hline $0.50 \cdot 0.99$ & 0.70 & 0.30 & 80799 & \\
\hline $1.00-1.99$ & 1.41 & 0.30 & 4084 & \\
\hline $2.00-4.99$ & 3.16 & 0.40 & 2384 & \\
\hline $5.00-9.99$ & 7.07 & 0.30 & 127 & \\
\hline$>10.00$ & 14.14 & 0.30 & 85 & \\
\hline \multicolumn{5}{|l|}{ Sample \#7 } \\
\hline \begin{tabular}{|l|}
$\begin{array}{l}\text { Bin } \\
\text { Range } \\
(\mu \dot{m})\end{array}$ \\
\end{tabular} & \begin{tabular}{|l} 
Bin Mean \\
Diameter \\
$(u m)$
\end{tabular} & $\begin{array}{l}\text { Diff. of the } \\
\text { log of the } \\
\text { Size }\end{array}$ & Counts & \begin{tabular}{|l|} 
Weighted \\
Mean \\
Diameter
\end{tabular} \\
\hline \begin{tabular}{|c|}
$0.30 \cdot 0.49$ \\
\end{tabular} & 0.38 & 0.21 & 127546 & \multirow{6}{*}{0.57} \\
\hline $0.50-0.99$ & 0.70 & 0.30 & 78904 & \\
\hline $1.00-1.99$ & 1.41 & 0.30 & 4539 & \\
\hline $2.00-4.99$ & 3.16 & 0.40 & 2839 & \\
\hline $5.00 \cdot 9.99$ & 7.07 & 0.30 & 153 & \\
\hline$>10.00$ & 14.14 & 0.30 & 122 & \\
\hline \multicolumn{5}{|l|}{ Sample \#9 } \\
\hline \begin{tabular}{|l}
$\begin{array}{l}\text { Bin } \\
\text { Range } \\
(\mu \mathrm{m})\end{array}$ \\
\end{tabular} & $\begin{array}{l}\text { Bin Mean } \\
\text { Diameter } \\
(\mathrm{mm})\end{array}$ & \begin{tabular}{|l|} 
Diff. of the \\
$\log$ of the \\
Size
\end{tabular} & Counts & $\begin{array}{l}\text { Weighted } \\
\text { Mean } \\
\text { Diameter }\end{array}$ \\
\hline $0.30 \cdot 0.49$ & 0.38 & 0.21 & 129057 & \multirow{6}{*}{0.57} \\
\hline $0.50-0.99$ & 0.70 & 0.30 & 80923 & \\
\hline $1.00-1.99$ & 1.41 & 0.30 & 4618 & \\
\hline $2.00-4.99$ & 3.16 & 0.40 & 2943 & \\
\hline $5.00-9.99$ & 7.07 & 0.30 & 154 & \\
\hline$>10.00$ & 14.14 & 0.30 & 119 & \\
\hline
\end{tabular}

Samole \#2

\begin{tabular}{|c|c|c|c|c|}
\hline $\begin{array}{l}\text { Bin } \\
\text { Range } \\
(u m)\end{array}$ & \begin{tabular}{|l} 
Bin Mean \\
Diameter \\
(um)
\end{tabular} & $\begin{array}{l}\text { Diff. of the } \\
\text { log of the } \\
\text { Size }\end{array}$ & Counts & $\begin{array}{l}\text { Weighted } \\
\text { Mean } \\
\text { Diameter }\end{array}$ \\
\hline $0.30-0.49$ & .38 & 0.21 & 132610 & \multirow{6}{*}{0.56} \\
\hline $0.50-0.99$ & .70 & 0.30 & 84420 & \\
\hline $1.00-1.99$ & 1.41 & 0.30 & 4476 & \\
\hline $2.00-4.99$ & 3.16 & 0.40 & 2596 & \\
\hline $5.00-9.99$ & 7.07 & 0.30 & 128 & \\
\hline$>10.00$ & 14.14 & 0.30 & 107 & \\
\hline
\end{tabular}

\begin{tabular}{|c|c|c|c|c|}
\hline \begin{tabular}{|l|} 
Bin \\
Range \\
$(\mu \mathrm{m})$
\end{tabular} & \begin{tabular}{|l|} 
Bin Mean \\
Diameter \\
$(\mu \mathrm{m})$
\end{tabular} & $\begin{array}{l}\text { Diff. of the } \\
\text { log of the } \\
\text { Size }\end{array}$ & Counts & $\begin{array}{l}\text { Weighted } \\
\text { Mean } \\
\text { Diameter }\end{array}$ \\
\hline $0.30-0.49$ & .38 & 0.21 & 130549 & \multirow{6}{*}{0.56} \\
\hline $0.50-0.99$ & .70 & 0.30 & 81277 & \\
\hline $1.00-1.99$ & 1.41 & 0.30 & 4161 & \\
\hline $2.00-4.99$ & 3.16 & 0.40 & 2506 & \\
\hline $5.00-9.99$ & 7.07 & 0.30 & 129 & \\
\hline$>10.00$ & 14.14 & 0.30 & 99 & \\
\hline
\end{tabular}

\begin{tabular}{||c|c|c|r|r||}
\hline $\begin{array}{l}\text { Bin } \\
\text { Range } \\
\text { (um) }\end{array}$ & $\begin{array}{l}\text { Bin Mean } \\
\text { Diameter } \\
(u \mathrm{~m})\end{array}$ & $\begin{array}{l}\text { Diff. of the } \\
\text { log of the } \\
\text { Size }\end{array}$ & Counts & $\begin{array}{l}\text { Weighted } \\
\text { Mean } \\
\text { Diameter }\end{array}$ \\
\hline $0.30-0.49$ & .38 & 0.21 & 130348 & \\
\hline $0.50-0.99$ & .70 & 0.30 & 81489 & \\
\hline $1.00-1.99$ & 1.41 & 0.30 & 4158 & \multirow{2}{*}{0.56} \\
\hline $2.00-4.99$ & 3.16 & 0.40 & 2405 & \\
\hline $5.00-9.99$ & 7.07 & 0.30 & 132 & \\
\hline$>10.00$ & 14.14 & 0.30 & 87 & \\
\hline
\end{tabular}

\begin{tabular}{|c|c|c|c|c|}
\hline \multicolumn{5}{|l|}{ Sample \#8 } \\
\hline $\begin{array}{l}\text { Bin } \\
\text { Range } \\
(\mu \mathrm{m})\end{array}$ & \begin{tabular}{|l} 
Bin Mean \\
Diameter \\
$(u m)$
\end{tabular} & $\begin{array}{l}\text { Diff. of the } \\
\log \text { of the } \\
\text { Size }\end{array}$ & Counts & \begin{tabular}{|l} 
Weighted \\
Mean \\
Diameter
\end{tabular} \\
\hline $0.30-0.49$ & .38 & 0.21 & 128324 & \multirow{6}{*}{0.58} \\
\hline $0.50-0.99$ & .70 & 0.30 & $\overline{81608}$ & \\
\hline $0.00-1.99$ & 1.41 & 0.30 & 5070 & \\
\hline $2.00-4.99$ & 3.16 & 0.40 & 3414 & \\
\hline $5.00-9.99$ & 7.07 & 0.30 & 203 & \\
\hline$>10.00$ & 14.14 & 0.30 & 154 & \\
\hline
\end{tabular}

\begin{tabular}{|c|c|c|c|c|}
\hline \multicolumn{5}{|c|}{ Sample $\# 10$} \\
\hline \begin{tabular}{|l} 
Bin \\
Range \\
$(\mu \mathrm{m})$
\end{tabular} & \begin{tabular}{|l|} 
Bin Mean \\
Diameter \\
$(\mu \mathrm{m})$
\end{tabular} & $\begin{array}{l}\text { Diff. of the } \\
\text { log of the } \\
\text { Size }\end{array}$ & Counts & $\begin{array}{l}\text { Weighted } \\
\text { Mean } \\
\text { Diameter }\end{array}$ \\
\hline $0.30-0.49$ & .38 & 0.21 & 126405 & \multirow{6}{*}{0.57} \\
\hline $0.50-0.99$ & .70 & 0.30 & 78385 & \\
\hline $1.00-1.99$ & 1.41 & 0.30 & 4335 & \\
\hline $2.00-4.99$ & 3.16 & 0.40 & 2898 & \\
\hline $5.00-9.99$ & 7.07 & 0.30 & 144 & \\
\hline$>10.00$ & 14.14 & 0.30 & 102 & \\
\hline
\end{tabular}




\section{Aerosol Particle Measurement Record}

Description of sample location 3706A Building, Ambient Air Test, at $1.5 \mathrm{~m}$, Date 8/9/94, Time sample started 9:46:48, Temperature 73.3 F, Humidity $43.7 \%$, Dew Point $51.4 \mathrm{~F}$, Average Wind Velocity (if applicable) $.04 \mathrm{~m} / \mathrm{s}$, \# of physical barriers between sample area and outside (if applicable) 1

\begin{tabular}{|c|c|c|c|c|}
\hline $\begin{array}{l}\text { Bin } \\
\text { Range } \\
(\mu \mathrm{m})\end{array}$ & $\begin{array}{l}\text { Bin Moan } \\
\text { Diameter } \\
(\mathrm{um})\end{array}$ & $\begin{array}{l}\text { Diff. of the } \\
\log \text { of the } \\
\text { Size }\end{array}$ & Counts & \begin{tabular}{|l} 
Weighted \\
Mean \\
Diameter
\end{tabular} \\
\hline $0.30 \cdot 0.49$ & 0.38 & 0.21 & 151795 & \multirow{6}{*}{0.58} \\
\hline $0.50-0.99$ & 0.70 & 0.30 & 103458 & \\
\hline $1.00-1.99$ & 1.41 & 0.30 & 5886 & \\
\hline $2.00-4.99$ & 3.16 & 0.40 & 3709 & \\
\hline $5.00-9.99$ & 7.07 & 0.30 & 202 & \\
\hline$>10.00$ & 14.14 & 0.30 & 108 & \\
\hline \multicolumn{5}{|l|}{ Sample \#3 } \\
\hline \begin{tabular}{|l} 
Bin \\
Range \\
(um)
\end{tabular} & $\begin{array}{l}\text { Bin Mean } \\
\text { Diameter } \\
(u \mathrm{~m})\end{array}$ & $\begin{array}{l}\text { Diff. of the } \\
\text { log of the } \\
\text { Size }\end{array}$ & Counts & $\begin{array}{l}\text { Weighted } \\
\text { Mean } \\
\text { Diameter }\end{array}$ \\
\hline $0.30-0.49$ & 0.38 & 0.21 & 150122 & \multirow{6}{*}{0.57} \\
\hline $0.50-0.99$ & 0.70 & 0.30 & 102678 & \\
\hline $1.00-1.99$ & 1.41 & 0.30 & 5253 & \\
\hline $2.00-4.99$ & 3.16 & 0.40 & 3284 & \\
\hline $5.00-9.99$ & 7.07 & 0.30 & 150 & \\
\hline$>10.00$ & 14.14 & 0.30 & 59 & \\
\hline \multicolumn{5}{|l|}{ Sample \#5 } \\
\hline \begin{tabular}{|l} 
Bin \\
Range \\
$(\mu \mathrm{m})$
\end{tabular} & $\begin{array}{l}\text { Bin Mean } \\
\text { Diameter } \\
(\mu \mathrm{m})\end{array}$ & \begin{tabular}{|l|} 
Diff. of the \\
log of the \\
Size
\end{tabular} & Counts & $\begin{array}{l}\text { Weighted } \\
\text { Mean } \\
\text { Diameter }\end{array}$ \\
\hline $0.30 \cdot 0.49$ & 0.38 & 0.21 & 141309 & \multirow{6}{*}{0.56} \\
\hline $0.50-0.99$ & 0.70 & 0.30 & 92081 & \\
\hline $1.00-1.99$ & 1.41 & 0.30 & 4780 & \\
\hline $2.00-4.99$ & 3.16 & 0.40 & 2728 & \\
\hline $5.00-9.99$ & 7.07 & 0.30 & 139 & \\
\hline$>10.00$ & 14.14 & 0.30 & 53 & \\
\hline \multicolumn{5}{|l|}{ Sample \#7 } \\
\hline $\begin{array}{l}\text { Bin } \\
\text { Range } \\
(\mu \mathrm{m}) \\
\end{array}$ & \begin{tabular}{|l} 
Bin Mean \\
Diameter \\
$(\mu \mathrm{m})$
\end{tabular} & \begin{tabular}{|l|} 
Diff. of the \\
log of the \\
Size
\end{tabular} & Counts & $\begin{array}{l}\text { Weighted } \\
\text { Mean } \\
\text { Diameter }\end{array}$ \\
\hline $0.30-0.49$ & 0.38 & 0.21 & 137457 & \multirow{6}{*}{0.56} \\
\hline $0.50 \cdot 0.99$ & 0.70 & 0.30 & 87303 & \\
\hline $1.00-1.99$ & 1.41 & 0.30 & 4463 & \\
\hline $2.00 \cdot 4.99$ & 3.16 & 0.40 & 2501 & \\
\hline $5.00-9.99$ & 7.07 & 0.30 & 120 & \\
\hline$>10.00$ & 14.14 & 0.30 & 39 & \\
\hline \multicolumn{5}{|l|}{ Sample \#9 } \\
\hline $\begin{array}{l}\text { Bin } \\
\text { Range } \\
(\mu \mathrm{m})\end{array}$ & \begin{tabular}{|l} 
Bin Mean \\
Diameter \\
$(\mu \mathrm{m})$
\end{tabular} & \begin{tabular}{|l|} 
Diff. of the \\
log of the \\
Size
\end{tabular} & Counts & $\begin{array}{l}\text { Weighted } \\
\text { Mean } \\
\text { Diameter }\end{array}$ \\
\hline $0.30 \cdot 0.49$ & 0.38 & 0.21 & 133055 & \multirow{6}{*}{0.56} \\
\hline $0.50-0.99$ & 0.70 & 0.30 & 84073 & \\
\hline $1.00-1.99$ & 1.41 & 0.30 & 4177 & \\
\hline $2.00-4.99$ & 3.16 & 0.40 & 2579 & \\
\hline $5.00-9.99$ & 7.07 & 0.30 & 126 & \\
\hline$>10.00$ & 14.14 & 0.30 & 44 & \\
\hline
\end{tabular}

\begin{tabular}{|c|c|c|c|c|}
\hline $\begin{array}{l}\text { Bin } \\
\text { Range } \\
(u m)\end{array}$ & \begin{tabular}{|l} 
Bin Mean \\
Diameter \\
$(u m)$
\end{tabular} & $\begin{array}{l}\text { Diff. of the } \\
\text { log of the } \\
\text { Size }\end{array}$ & Counts & $\begin{array}{l}\text { Weighted } \\
\text { Mean } \\
\text { Diameter }\end{array}$ \\
\hline $0.30-0.49$ & .38 & 0.21 & 159531 & \multirow{6}{*}{0.56} \\
\hline $0.50-0.99$ & .70 & 0.30 & 106225 & \\
\hline $1.00-1.99$ & 1.41 & 0.30 & 5389 & \\
\hline $2.00-4.99$ & 3.16 & 0.40 & 3327 & \\
\hline $5.00-9.99$ & 7.07 & 0.30 & 158 & \\
\hline$>10.00$ & 14.14 & 0.30 & 69 & \\
\hline \multicolumn{5}{|c|}{ Sample \#4 } \\
\hline $\begin{array}{l}\text { Bin } \\
\text { Range } \\
(\mathrm{mm}) \\
\end{array}$ & \begin{tabular}{|l} 
Bin Mean \\
Diameter \\
$(\mu \mathrm{m})$
\end{tabular} & $\begin{array}{l}\text { Diff. of the } \\
\text { log of the } \\
\text { Size }\end{array}$ & Counts & $\begin{array}{l}\text { Weighted } \\
\text { Mean } \\
\text { Diameter }\end{array}$ \\
\hline $0.30-0.49$ & .38 & 0.21 & 148742 & \multirow{6}{*}{0.57} \\
\hline $0.50-0.99$ & .70 & 0.30 & 98958 & \\
\hline $1.00-1.99$ & 1.41 & 0.30 & 5142 & \\
\hline $2.00-4.99$ & 3.16 & 0.40 & 3055 & \\
\hline $5.00-9.99$ & 7.07 & 0.30 & 170 & \\
\hline$>10.00$ & $\overline{14.14}$ & 0.30 & 71 & \\
\hline
\end{tabular}

\begin{tabular}{|c|c|c|c|c|}
\hline \multicolumn{5}{|l|}{ Sample \#6 } \\
\hline $\begin{array}{l}\text { Bin } \\
\text { Range } \\
(\mathrm{mm})\end{array}$ & \begin{tabular}{|l} 
Bin Mean \\
Diameter \\
$(\mu \mathrm{m})$
\end{tabular} & $\begin{array}{l}\text { Diff. of the } \\
\text { log of the } \\
\text { Size }\end{array}$ & Counts & $\begin{array}{l}\text { Weighted } \\
\text { Mean } \\
\text { Diameter }\end{array}$ \\
\hline $0.30-0.49$ & .38 & 0.21 & 140734 & \multirow{6}{*}{0.56} \\
\hline $0.50-0.99$ & .70 & 0.30 & $\overline{91642}$ & \\
\hline $1.00-1.99$ & 1.41 & 0.30 & 4464 & \\
\hline $2.00-4.99$ & 3.16 & 0.40 & 2705 & \\
\hline $5.00-9.99$ & 7.07 & 0.30 & 142 & \\
\hline$>10.00$ & 14.14 & 0.30 & 40 & \\
\hline
\end{tabular}

\begin{tabular}{|c|c|c|c|c|}
\hline $\begin{array}{l}\text { Bin } \\
\text { Range } \\
(u m)\end{array}$ & \begin{tabular}{|l|} 
Bin Mean \\
Diameter \\
$(u m)$
\end{tabular} & $\begin{array}{l}\text { Diff. of the } \\
\text { log of the } \\
\text { Size }\end{array}$ & Counts & \begin{tabular}{|l} 
Weighted \\
Mean \\
Diameter
\end{tabular} \\
\hline $0.30-0.49$ & .38 & 0.21 & 137833 & \multirow{6}{*}{0.56} \\
\hline $0.50-0.99$ & .70 & 0.30 & $\overline{89412}$ & \\
\hline $0.00-1.99$ & 1.41 & 0.30 & 4769 & \\
\hline $2.00-4.99$ & 3.16 & 0.40 & 2860 & \\
\hline $5.00-9.99$ & 7.07 & 0.30 & 144 & \\
\hline$>10.00$ & $\overline{14.14}$ & 0.30 & 68 & \\
\hline
\end{tabular}

\begin{tabular}{|c|c|c|c|c|}
\hline \multicolumn{5}{|c|}{ Sample \#10 } \\
\hline \begin{tabular}{|l|} 
Bin \\
Range \\
(um)
\end{tabular} & \begin{tabular}{|l} 
Bin Mean \\
Diameter \\
$(u m)$
\end{tabular} & $\begin{array}{l}\text { Diff. of the } \\
\text { log of the } \\
\text { Size }\end{array}$ & Counts & $\begin{array}{l}\text { Weighted } \\
\text { Mean } \\
\text { Diameter }\end{array}$ \\
\hline $0.30-0.49$ & .38 & 0.21 & 130546 & \multirow{6}{*}{0.56} \\
\hline $0.50-0.99$ & .70 & 0.30 & 82533 & \\
\hline $1.00-1.99$ & 1.41 & 0.30 & 4182 & \\
\hline $2.00-4.99$ & 3.16 & 0.40 & 2444 & \\
\hline $5.00-9.99$ & 7.07 & 0.30 & 128 & \\
\hline$>10.00$ & 14.14 & 0.30 & 64 & \\
\hline
\end{tabular}




\section{Aerosol Particle Measurement Record}

Description of sample location 3706 A Building, Ambient Air Test, at $2 \mathrm{~m}$, Date 8/9/94, Time sample started 11:05:47, Temperature 76.3 F, Humidity 40.1\%, Dew Point 51.1 F - Average Wind Velocity (if applicable) $.00 \mathrm{~m} / \mathrm{s}$, \# of physical barriers between sample area and outside (if applicable) 1

\begin{tabular}{|c|c|c|c|c|}
\hline $\begin{array}{l}\text { Bin } \\
\text { Range } \\
(\mu \mathrm{m})\end{array}$ & $\begin{array}{l}\text { Bin Mean } \\
\text { Diameter } \\
(\mathrm{wm})\end{array}$ & $\begin{array}{l}\text { Diff. of the } \\
\text { log of the } \\
\text { Size }\end{array}$ & Counts & $\begin{array}{l}\text { Weighted } \\
\text { Mean } \\
\text { Diameter }\end{array}$ \\
\hline $0.30-0.49$ & \begin{tabular}{|l|}
0.38 \\
\end{tabular} & 0.21 & 105692 & \multirow{6}{*}{0.60} \\
\hline $0.50-0.99$ & 0.70 & 0.30 & 69462 & \\
\hline $1.00-1.99$ & 1.41 & 0.30 & 4968 & \\
\hline $2.00 \cdot 4.99$ & 3.16 & 0.40 & 3173 & \\
\hline $5.00-9.99$ & 7.07 & 0.30 & 199 & \\
\hline$>10.00$ & 14.14 & 0.30 & 168 & \\
\hline \multicolumn{5}{|l|}{ Sample \#3 } \\
\hline \begin{tabular}{|l} 
Bin \\
Range \\
$(u m)$
\end{tabular} & \begin{tabular}{|l} 
Bin Mean \\
Diameter \\
$(u m)$
\end{tabular} & $\begin{array}{l}\text { Diff. of the } \\
\text { log of the } \\
\text { Size }\end{array}$ & Counts & $\begin{array}{l}\text { Weighted } \\
\text { Mean } \\
\text { Diameter }\end{array}$ \\
\hline $0.30-0.49$ & 0.38 & 0.21 & 106030 & \multirow{6}{*}{0.61} \\
\hline $0.50 \cdot 0.99$ & 0.70 & 0.30 & 70155 & \\
\hline $1.00-1.99$ & 1.41 & 0.30 & 5130 & \\
\hline $2.00-4.99$ & 3.16 & 0.40 & 3371 & \\
\hline $5.00 \cdot 9.99$ & 7.07 & 0.30 & 246 & \\
\hline$>10.00$ & 14.14 & 0.30 & 283 & \\
\hline \multicolumn{5}{|l|}{ Sample \#5 } \\
\hline \begin{tabular}{|l} 
Bin \\
Range \\
$(\mu \mathrm{m})$
\end{tabular} & $\begin{array}{l}\text { Bin Mean } \\
\text { Diameter } \\
(\mu \mathrm{m})\end{array}$ & $\begin{array}{l}\text { Diff. of the } \\
\log \text { of the } \\
\text { Size }\end{array}$ & Counts & $\begin{array}{l}\text { Weighted } \\
\text { Mean } \\
\text { Diameter }\end{array}$ \\
\hline $0.30-0.49$ & 0.38 & 0.21 & 101179 & \multirow{6}{*}{0.63} \\
\hline $0.50-0.99$ & 0.70 & 0.30 & 68550 & \\
\hline $1.00-1.99$ & 1.41 & 0.30 & 5372 & \\
\hline $2.00-4.99$ & 3.16 & 0.40 & 3505 & \\
\hline $5.00-9.99$ & 7.07 & 0.30 & 254 & \\
\hline$>10.00$ & 14.14 & 0.30 & 460 & \\
\hline \multicolumn{5}{|l|}{ Sample \#7 } \\
\hline $\begin{array}{l}\text { Bin } \\
\text { Range } \\
\text { (um) } \\
\end{array}$ & \begin{tabular}{|l|} 
Bin Mean \\
Diameter \\
$(\mu \mathrm{m})$
\end{tabular} & $\begin{array}{l}\text { Diff. of the } \\
\text { log of the } \\
\text { Size }\end{array}$ & Counts & $\begin{array}{l}\text { Weighted } \\
\text { Mean } \\
\text { Diameter }\end{array}$ \\
\hline $0.30-0.49$ & 0.38 & 0.21 & 101154 & \multirow{6}{*}{0.60} \\
\hline $0.50-0.99$ & 0.70 & 0.30 & 65890 & \\
\hline $1.00-1.99$ & 1.41 & 0.30 & 4581 & \\
\hline $2.00 \cdot 4.99$ & 3.16 & 0.40 & 2863 & \\
\hline $5.00-9.99$ & 7.07 & 0.30 & 195 & \\
\hline$>10.00$ & 14.14 & 0.30 & 225 & \\
\hline \multicolumn{5}{|l|}{ Sample \#9 } \\
\hline $\begin{array}{l}\text { Bin } \\
\text { Pange } \\
(\mu \mathrm{m})\end{array}$ & $\begin{array}{l}\text { Bin Mean } \\
\text { Diameter } \\
\text { (um) }\end{array}$ & $\begin{array}{l}\text { Diff. of the } \\
\text { log of the } \\
\text { Size }\end{array}$ & Counts & \begin{tabular}{|l} 
Weighted \\
Mean \\
Diameter
\end{tabular} \\
\hline $0.30-0.49$ & $\begin{array}{l}0.38 \\
\end{array}$ & 0.21 & 99981 & \multirow{6}{*}{0.59} \\
\hline $0.50 \cdot 0.99$ & 0.70 & 0.30 & 63165 & \\
\hline $1.00-1.99$ & 1.41 & 0.30 & 4296 & \\
\hline $2.00-4.99$ & 3.16 & 0.40 & 2791 & \\
\hline $5.00-9.99$ & 7.07 & 0.30 & 174 & \\
\hline$>10.00$ & 14.14 & 0.30 & 188 & \\
\hline
\end{tabular}

Sample \#2

\begin{tabular}{|c|c|c|c|c|}
\hline $\begin{array}{l}\text { Bin } \\
\text { Range } \\
(\mu m)\end{array}$ & $\begin{array}{l}\text { Bin Mean } \\
\text { Diameter } \\
(u m)\end{array}$ & $\begin{array}{l}\text { Diff. of the } \\
\text { log of the } \\
\text { Size }\end{array}$ & Counts & $\begin{array}{l}\text { Weighted } \\
\text { Mean } \\
\text { Diameter }\end{array}$ \\
\hline $0.30-0.49$ & .38 & 0.21 & 106445 & \multirow{6}{*}{0.60} \\
\hline $0.50-0.99$ & .70 & 0.30 & 70101 & \\
\hline $1.00-1.99$ & 1.41 & 0.30 & 4954 & \\
\hline $2.00-4.99$ & 3.16 & 0.40 & 3253 & \\
\hline $5.00-9.99$ & 7.07 & 0.30 & 217 & \\
\hline$>10.00$ & 14.14 & 0.30 & 172 & \\
\hline
\end{tabular}

\begin{tabular}{|c|c|c|c|c|}
\hline $\begin{array}{l}\text { Bin } \\
\text { Range } \\
\text { (um) }\end{array}$ & $\begin{array}{l}\text { Bin Mean } \\
\text { Diameter } \\
(\mathrm{um})\end{array}$ & $\begin{array}{l}\text { Diff. of the } \\
\text { log of the } \\
\text { Size }\end{array}$ & Counts & $\begin{array}{l}\text { Weighted } \\
\text { Mean } \\
\text { Diameter }\end{array}$ \\
\hline $0.30-0.49$ & .38 & 0.21 & 101749 & \multirow{6}{*}{0.62} \\
\hline $0.50-0.99$ & .70 & 0.30 & 66965 & \\
\hline $1.00-1.99$ & 1.41 & 0.30 & 4916 & \\
\hline $2.00-4.99$ & 3.16 & 0.40 & 3360 & \\
\hline $5.00-9.99$ & 7.07 & 0.30 & 255 & \\
\hline$>10.00$ & 14.14 & 0.30 & 347 & \\
\hline
\end{tabular}

\begin{tabular}{|c|c|c|c|c|}
\hline $\begin{array}{l}\text { Bin } \\
\text { Range } \\
\text { (um) }\end{array}$ & $\begin{array}{l}\text { Bin Mean } \\
\text { Diameter } \\
(\mathrm{um})\end{array}$ & $\begin{array}{l}\text { Diff. of the } \\
\text { log of the } \\
\text { Size }\end{array}$ & Counts & \begin{tabular}{|l} 
Weighted \\
Mean \\
Diameter
\end{tabular} \\
\hline $0.30-0.49$ & .38 & 0.21 & 100799 & \multirow{6}{*}{0.61} \\
\hline $0.50-0.99$ & .70 & 0.30 & 66000 & \\
\hline $1.00-1.99$ & 1.41 & 0.30 & 4983 & \\
\hline $2.00-4.99$ & 3.16 & 0.40 & 3154 & \\
\hline $5.00-9.99$ & 7.07 & 0.30 & 206 & \\
\hline$>10.00$ & 14.14 & 0.30 & 274 & \\
\hline
\end{tabular}

\begin{tabular}{|c|c|c|c|c|}
\hline \multicolumn{5}{|c|}{ Sample \#8 } \\
\hline $\begin{array}{l}\text { Bin } \\
\text { Range } \\
(u m)\end{array}$ & $\begin{array}{l}\text { Bin Mean } \\
\text { Diameter } \\
(\mu \mathrm{m})\end{array}$ & $\begin{array}{l}\text { Diff. of the } \\
\text { log of the } \\
\text { Size }\end{array}$ & Counts & $\begin{array}{l}\text { Weighted } \\
\text { Moan } \\
\text { Diameter }\end{array}$ \\
\hline $0.30-0.49$ & .38 & 0.21 & 100069 & \multirow{6}{*}{0.60} \\
\hline $0.50-0.99$ & .70 & 0.30 & 66139 & \\
\hline $0.00-1.99$ & 1.41 & 0.30 & 4772 & \\
\hline $2.00-4.99$ & 3.16 & 0.40 & 3104 & \\
\hline $5.00-9.99$ & 7.07 & 0.30 & 196 & \\
\hline$>10.00$ & 14.14 & 0.30 & 225 & \\
\hline
\end{tabular}

\begin{tabular}{|c|c|c|c|c|}
\hline \multicolumn{5}{|c|}{ Sample $\# 10$} \\
\hline $\begin{array}{l}\text { Bin } \\
\text { Range } \\
(\mathrm{um})\end{array}$ & \begin{tabular}{|l} 
Bin Mean \\
Diameter \\
$(\mu \mathrm{m})$
\end{tabular} & $\begin{array}{l}\text { Diff. of the } \\
\text { log of the } \\
\text { Size }\end{array}$ & Counts & $\begin{array}{l}\text { Weighted } \\
\text { Moan } \\
\text { Diameter }\end{array}$ \\
\hline $0.30-0.49$ & .38 & 0.21 & 99117 & \multirow{6}{*}{0.58} \\
\hline $0.50-0.99$ & .70 & 0.30 & 62537 & \\
\hline $1.00-1.99$ & 1.41 & 0.30 & 4133 & \\
\hline $2.00-4.99$ & 3.16 & 0.40 & 2622 & \\
\hline $5.00-9.99$ & 7.07 & 0.30 & 169 & \\
\hline$>10.00$ & 14.14 & 0.30 & 133 & \\
\hline
\end{tabular}


Data for Table 4. Mean Optical Diameter of Ambient Air, Building 3706 Room 102,

and

Fig. 1 Ambient Air Vs. Sample Height, Building 3706, Rm 102

$2 \mathrm{~cm} .(0.02$ meters)

0.5 meters

1.0 meters

1.5 meters

2.0 meters 


\section{Aerosol Particle Measurement Record}

Description of sample location 3706 Building, Room 102, Ambient Air Test, at $2 \mathrm{~cm}$, Date 7/27/94, Time sample started 11:36:00, Temperature 76.0 F, Humidity $40.1 \%$, Dew Point $50.0 \mathrm{~F}$, Average Wind Velocity (if applicable) $.00 \mathrm{~m} / \mathrm{s}$, \# of physical barriers between sample area and outside (if applicable). 3 ,

Sample \#1

\begin{tabular}{|c|c|c|c|c|}
\hline $\begin{array}{l}\text { Bin } \\
\text { Range } \\
(\mu \mathrm{m})\end{array}$ & \begin{tabular}{|l|} 
Bin Mean \\
Diameter \\
$(\mu \mathrm{m})$
\end{tabular} & $\begin{array}{l}\text { Diff. of the } \\
\text { log of the } \\
\text { Size }\end{array}$ & Counts & $\begin{array}{l}\text { Weighted } \\
\text { Mean } \\
\text { Diameter }\end{array}$ \\
\hline $0.30 \cdot 0.49$ & 0.38 & 0.21 & 147838 & \multirow{6}{*}{0.65} \\
\hline $0.50-0.99$ & 0.70 & 0.30 & 46422 & \\
\hline $1.00-1.99$ & 1.41 & 0.30 & 10610 & \\
\hline $2.00-4.99$ & 3.16 & 0.40 & 8089 & \\
\hline $5.00-9.99$ & 7.07 & 0.30 & 544 & \\
\hline$>10.00$ & 14.14 & 0.30 & 458 & \\
\hline \multicolumn{5}{|l|}{ Sample \#3 } \\
\hline \begin{tabular}{|l} 
Bin \\
Range \\
$(\mu \mathrm{m})$
\end{tabular} & \begin{tabular}{|l}
$\begin{array}{l}\text { Bin Mean } \\
\text { Diameter } \\
(\mu \mathrm{m})\end{array}$ \\
\end{tabular} & $\begin{array}{l}\text { Diff. of the } \\
\text { log of the } \\
\text { Size }\end{array}$ & Counts & $\begin{array}{l}\text { Weighted } \\
\text { Mean } \\
\text { Diameter }\end{array}$ \\
\hline $0.30-0.49$ & 0.38 & 0.21 & 146376 & \multirow{6}{*}{0.67} \\
\hline $0.50-0.99$ & 0.70 & 0.30 & 46392 & \\
\hline $1.00-1.99$ & 1.41 & 0.30 & 10684 & \\
\hline $2.00-4.99$ & 3.16 & 0.40 & 8299 & \\
\hline $5.00-9.99$ & 7.07 & 0.30 & 616 & \\
\hline$>10.00$ & 14.14 & 0.30 & 636 & \\
\hline \multicolumn{5}{|l|}{ Sample \#5 } \\
\hline \begin{tabular}{|l} 
Bin \\
Range \\
$(\mu \mathrm{m})$ \\
\end{tabular} & \begin{tabular}{|l} 
Bin Mean \\
Diameter \\
$(\mu \mathrm{m})$
\end{tabular} & \begin{tabular}{|l|} 
Diff. of the \\
log of the \\
Size
\end{tabular} & Counts & $\begin{array}{l}\text { Weighted } \\
\text { Mean } \\
\text { Diameter }\end{array}$ \\
\hline $0.30 \cdot 0.49$ & 0.38 & 0.21 & 149374 & \multirow{6}{*}{0.64} \\
\hline $0.50-0.99$ & 0.70 & 0.30 & 46810 & \\
\hline $1.00 \cdot 1.99$ & 1.41 & 0.30 & 10437 & \\
\hline $2.00 \cdot 4.99$ & 3.16 & 0.40 & 7751 & \\
\hline $5.00-9.99$ & 7.07 & 0.30 & 496 & \\
\hline$>10.00$ & 14.14 & 0.30 & 379 & \\
\hline \multicolumn{5}{|l|}{ Sample \#7 } \\
\hline $\begin{array}{l}\text { Bin } \\
\text { Range } \\
(\mu \mathrm{m})\end{array}$ & $\begin{array}{l}\text { Bin Mean } \\
\text { Diameter } \\
(\mu \mathrm{m})\end{array}$ & \begin{tabular}{|l|} 
Diff. of the \\
log of the \\
Size
\end{tabular} & Counts & \begin{tabular}{|l|} 
Weighted \\
Mean \\
Diameter
\end{tabular} \\
\hline $0.30 \cdot 0.49$ & \begin{tabular}{|l|}
0.38 \\
\end{tabular} & 0.21 & 150586 & \multirow{6}{*}{0.63} \\
\hline $0.50 \cdot 0.99$ & 0.70 & 0.30 & 46766 & \\
\hline $1.00-1.99$ & 1.41 & 0.30 & 10417 & \\
\hline $2.00-4.99$ & 3.16 & 0.40 & 7420 & \\
\hline $5.00-9.99$ & 7.07 & 0.30 & 432 & \\
\hline$>10.00$ & 14.14 & 0.30 & 337 & \\
\hline \multicolumn{5}{|l|}{ ample \#9 } \\
\hline $\begin{array}{l}\text { Bin } \\
\text { Range } \\
(\mu \mathrm{m}) \\
\end{array}$ & $\begin{array}{l}\text { Bin Mean } \\
\text { Diameter } \\
(\mu \mathrm{m})\end{array}$ & $\begin{array}{l}\text { Diff. of the } \\
\text { log of the } \\
\text { Size }\end{array}$ & Counts & $\begin{array}{l}\text { Weighted } \\
\text { Mean } \\
\text { Diameter }\end{array}$ \\
\hline $0.30-0.49$ & 0.38 & 0.21 & 149762 & \multirow{6}{*}{0.65} \\
\hline $0.50 \cdot 0.99$ & 0.70 & 0.30 & 47689 & \\
\hline $1.00-1.99$ & 1.41 & 0.30 & 10748 & \\
\hline $2.00 \cdot 4.99$ & 3.16 & 0.40 & 8130 & \\
\hline $5.00-9.99$ & 7.07 & 0.30 & 562 & \\
\hline$>10.00$ & 14.14 & 0.30 & 396 & \\
\hline
\end{tabular}

Sample \#2

\begin{tabular}{|c|c|c|c|c|}
\hline $\begin{array}{l}\text { Bin } \\
\text { Range } \\
\text { (um) }\end{array}$ & \begin{tabular}{|l} 
Bin Mean \\
Diameter \\
(urm)
\end{tabular} & $\begin{array}{l}\text { Diff. of the } \\
\text { log of the } \\
\text { Size }\end{array}$ & Counts & $\begin{array}{l}\text { Weighted } \\
\text { Mean } \\
\text { Diameter }\end{array}$ \\
\hline $0.30-0.49$ & .38 & 0.21 & 146945 & \multirow{6}{*}{0.66} \\
\hline $0.50-0.99$ & .70 & 0.30 & 46174 & \\
\hline $1.00-1.99$ & 1.41 & 0.30 & 10581 & \\
\hline $2.00-4.99$ & 3.16 & 0.40 & 7955 & \\
\hline $5.00-9.99$ & 7.07 & 0.30 & 508 & \\
\hline$>10.00$ & 14.14 & 0.30 & 581 & \\
\hline
\end{tabular}

Sample \#4

\begin{tabular}{|c|c|c|c|c|}
\hline \begin{tabular}{|l} 
Bin \\
Range \\
$(u \mathrm{~m})$
\end{tabular} & \begin{tabular}{|l|} 
Bin Mean \\
Diameter \\
$(u \mathrm{~m})$
\end{tabular} & $\begin{array}{l}\text { Diff. of the } \\
\log \text { of the } \\
\text { Size }\end{array}$ & Counts & $\begin{array}{l}\text { Weighted } \\
\text { Mean } \\
\text { Diameter }\end{array}$ \\
\hline $0.30-0.49$ & .38 & 0.21 & 147719 & \multirow{6}{*}{0.65} \\
\hline $0.50-0.99$ & .70 & 0.30 & 47237 & \\
\hline $1.00-1.99$ & 1.41 & 0.30 & 10605 & \\
\hline $2.00-4.99$ & 3.16 & 0.40 & 7895 & \\
\hline $5.00-9.99$ & 7.07 & 0.30 & 579 & \\
\hline$>10.00$ & 14.14 & 0.30 & 435 & \\
\hline
\end{tabular}

\begin{tabular}{|c|c|c|c|c|}
\hline \multicolumn{5}{|l|}{ Sample \#6 } \\
\hline \begin{tabular}{|l} 
Bin \\
Range \\
$(\mu \mathrm{m})$
\end{tabular} & \begin{tabular}{|l} 
Bin Mean \\
Diameter \\
$(\mu \mathrm{m})$
\end{tabular} & $\begin{array}{l}\text { Diff. of the } \\
\log \text { of the } \\
\text { Size }\end{array}$ & Counts & $\begin{array}{l}\text { Weighted } \\
\text { Mean } \\
\text { Diameter }\end{array}$ \\
\hline $0.30-0.49$ & .38 & 0.21 & 149800 & \multirow{6}{*}{0.63} \\
\hline $0.50-0.99$ & .70 & 0.30 & 46772 & \\
\hline $1.00-1.99$ & 1.41 & 0.30 & 10645 & \\
\hline $2.00-4.99$ & 3.16 & 0.40 & 7608 & \\
\hline $5.00-9.99$ & 7.07 & 0.30 & 476 & \\
\hline$>10.00$ & 14.14 & 0.30 & 301 & \\
\hline
\end{tabular}

Sample \#8

\begin{tabular}{|c|c|c|c|c|}
\hline $\begin{array}{l}\text { Bin } \\
\text { Range } \\
(\mu \mathrm{m})\end{array}$ & \begin{tabular}{|l|} 
Bin Mean \\
Diameter \\
$(u \mathrm{~m})$
\end{tabular} & $\begin{array}{l}\text { Diff. of the } \\
\text { log of the } \\
\text { Size }\end{array}$ & Counts & $\begin{array}{l}\text { Weighted } \\
\text { Mean } \\
\text { Diameter }\end{array}$ \\
\hline $0.30-0.49$ & .38 & 0.21 & 149295 & \multirow{6}{*}{0.64} \\
\hline $0.50-0.99$ & .70 & 0.30 & 47337 & \\
\hline $0.00-1.99$ & 1.41 & 0.30 & 10662 & \\
\hline $2.00-4.99$ & 3.16 & 0.40 & 7811 & \\
\hline $5.00-9.99$ & 7.07 & 0.30 & 458 & \\
\hline$>10.00$ & $\overline{14.14}$ & 0.30 & 320 & \\
\hline
\end{tabular}

\begin{tabular}{|c|c|c|c|c|}
\hline \multicolumn{5}{|c|}{ Sample \#10 } \\
\hline $\begin{array}{l}\text { Bin } \\
\text { Range } \\
(u m)\end{array}$ & \begin{tabular}{|l|} 
Bin Mean \\
Diameter \\
$(\mathrm{hm})$
\end{tabular} & $\begin{array}{l}\text { Diff. of the } \\
\text { log of the } \\
\text { Size }\end{array}$ & Counts & $\begin{array}{l}\text { Weighted } \\
\text { Mean } \\
\text { Diameter }\end{array}$ \\
\hline $0.30-0.49$ & .38 & 0.21 & 150398 & \multirow{6}{*}{0.64} \\
\hline $0.50-0.99$ & .70 & 0.30 & 47439 & \\
\hline $1.00-1.99$ & 1.41 & 0.30 & $\overline{10836}$ & \\
\hline $2.00-4.99$ & 3.16 & 0.40 & 8077 & \\
\hline $5.00-9.99$ & 7.07 & 0.30 & 526 & \\
\hline$>10.00$ & 14.14 & 0.30 & 363 & \\
\hline
\end{tabular}




\section{Aerosol Particle Measurement Record}

Description of sample location 3706 Building, Room 102, Ambient Air Test, at 0.5 meter, Date 7/27/94, Time sample started 12:36:00, Temperature 78.5 F, Humidity 39.5\%, Dew Point $50.7 \mathrm{~F}$, Average Wind Velocity (if applicable) $.00 \mathrm{~m} / \mathrm{s}$, \# of physical barriers between sample area and outside (if applicable). $\underline{3}$,

\begin{tabular}{|c|c|c|c|c|}
\hline \begin{tabular}{|l} 
Bin \\
Range \\
(um)
\end{tabular} & $\begin{array}{l}\text { Bin Mean } \\
\text { Diameter } \\
(\mathrm{erm})\end{array}$ & $\begin{array}{l}\text { Diff. of the } \\
\log \text { of the } \\
\text { Size }\end{array}$ & Counts & $\begin{array}{l}\text { Weighted } \\
\text { Mean } \\
\text { Diameter }\end{array}$ \\
\hline $0.30-0.49$ & 0.38 & 0.21 & 145158 & \multirow{6}{*}{0.63} \\
\hline $0.50-0.99$ & 0.70 & 0.30 & 44835 & \\
\hline $1.00-1.99$ & 1.41 & 0.30 & 9923 & \\
\hline $2.00-4.99$ & 3.16 & 0.40 & 7083 & \\
\hline $5.00-9.99$ & 7.07 & 0.30 & 450 & \\
\hline$>10.00$ & 14.14 & 0.30 & 399 & \\
\hline \multicolumn{5}{|l|}{ Sample \#3 } \\
\hline \begin{tabular}{|l} 
Bin \\
Range \\
$(\mu \mathrm{m})$
\end{tabular} & \begin{tabular}{|l|} 
Bin Mean \\
Diameter \\
$(\mu \mathrm{m})$
\end{tabular} & $\begin{array}{l}\text { Diff. of the } \\
\log \text { of the } \\
\text { Size }\end{array}$ & Counts & $\begin{array}{l}\text { Weighted } \\
\text { Mean } \\
\text { Diameter }\end{array}$ \\
\hline $0.30 \cdot 0.49$ & 0.38 & 0.21 & 145460 & \multirow{6}{*}{0.63} \\
\hline $0.50 \cdot 0.99$ & 0.70 & 0.30 & 44801 & \\
\hline $1.00-1.99$ & 1.41 & 0.30 & 9922 & \\
\hline $2.00-4.99$ & 3.16 & 0.40 & 6817 & \\
\hline $5.00-9.99$ & 7.07 & 0.30 & 409 & \\
\hline$>10.00$ & 14.14 & 0.30 & 374 & \\
\hline \multicolumn{5}{|l|}{ Sample \#5 } \\
\hline \begin{tabular}{|l} 
Bin \\
Range \\
$(\mu \mathrm{m})$ \\
\end{tabular} & \begin{tabular}{|l}
$\begin{array}{l}\text { Bin Mean } \\
\text { Diameter } \\
(\mu \mathrm{m})\end{array}$ \\
\end{tabular} & \begin{tabular}{|l|} 
Diff. of the \\
log of the \\
Size
\end{tabular} & Counts & $\begin{array}{l}\text { Weighted } \\
\text { Mean } \\
\text { Diameter }\end{array}$ \\
\hline \begin{tabular}{|c|}
$0.30-0.49$ \\
\end{tabular} & 0.38 & 0.21 & 147741 & \multirow{6}{*}{0.61} \\
\hline $0.50-0.99$ & 0.70 & 0.30 & 44757 & \\
\hline $1.00-1.99$ & 1.41 & 0.30 & 9635 & \\
\hline $2,00-4.99$ & 3.16 & 0.40 & 6583 & \\
\hline $5.00-9.99$ & 7.07 & 0.30 & 367 & \\
\hline$>10.00$ & 14.14 & 0.30 & 215 & \\
\hline \multicolumn{5}{|l|}{ Sample $\# 7$} \\
\hline \begin{tabular}{|l|} 
Bin \\
Range \\
(um)
\end{tabular} & \begin{tabular}{|l|} 
Bin Mean \\
Diameter \\
(um)
\end{tabular} & \begin{tabular}{|l|} 
Diff. of the \\
log of the \\
Size
\end{tabular} & Counts & \begin{tabular}{|l|} 
Weighted \\
Mean \\
Diameter
\end{tabular} \\
\hline $0.30 \cdot 0.49$ & 0.38 & 0.21 & 148412 & \multirow{6}{*}{0.61} \\
\hline $0.50-0.99$ & 0.70 & 0.30 & 45109 & \\
\hline $1.00-1.99$ & 1.41 & 0.30 & 9787 & \\
\hline $2.00-4.99$ & 3.16 & 0.40 & 6608 & \\
\hline $5.00-9.99$ & 7.07 & 0.30 & 385 & \\
\hline$>10.00$ & 14.14 & 0.30 & 288 & \\
\hline \multicolumn{5}{|l|}{ Sample \#9 } \\
\hline $\begin{array}{l}\text { Bin } \\
\text { Range } \\
(\mu \mathrm{m})\end{array}$ & \begin{tabular}{|l|}
$\begin{array}{l}\text { Bin Mean } \\
\text { Diameter } \\
(u m)\end{array}$ \\
\end{tabular} & \begin{tabular}{|l|} 
Diff. of the \\
log of the \\
Size
\end{tabular} & Counts & \begin{tabular}{|l|} 
Weighted \\
Mean \\
Diameter
\end{tabular} \\
\hline $0.30-0.49$ & $\begin{array}{l}0.38 \\
\end{array}$ & 0.21 & 147563 & \multirow{6}{*}{0.64} \\
\hline $0.50-0.99$ & 0.70 & 0.30 & 45200 & \\
\hline $1.00-1.99$ & 1.41 & 0.30 & 9825 & \\
\hline $2.00 \cdot 4.99$ & 3.16 & 0.40 & 7136 & \\
\hline $5.00-9.99$ & 7.07 & 0.30 & 424 & \\
\hline$>10.00$ & 14.14 & 0.30 & 493 & \\
\hline
\end{tabular}

Sample \#2

\begin{tabular}{|c|c|c|c|c|}
\hline $\begin{array}{l}\text { Bin } \\
\text { Range } \\
\text { ( } / \mathrm{m})\end{array}$ & \begin{tabular}{|l} 
Bin Mean \\
Diameter \\
$(\mathrm{erm})$
\end{tabular} & $\begin{array}{l}\text { Diff. of the } \\
\log \text { of the } \\
\text { Size }\end{array}$ & Counts & $\begin{array}{l}\text { Weighted } \\
\text { Mean } \\
\text { Diameter }\end{array}$ \\
\hline $0.30-0.49$ & .38 & 0.21 & 144015 & \multirow{6}{*}{0.65} \\
\hline $0.50-0.99$ & .70 & 0.30 & 44760 & \\
\hline $1.00-1.99$ & 1.41 & 0.30 & 9977 & \\
\hline $2.00-4.99$ & 3.16 & 0.40 & 7152 & \\
\hline $5.00-9.99$ & 7.07 & 0.30 & 487 & \\
\hline$>10.00$ & 14.14 & 0.30 & 606 & \\
\hline
\end{tabular}

Sample \#4

\begin{tabular}{|c|c|c|c|c|}
\hline $\begin{array}{l}\text { Bin } \\
\text { Range } \\
(\mu \mathrm{m})\end{array}$ & \begin{tabular}{|l} 
Bin Mean \\
Diameter \\
$(\mathrm{um})$
\end{tabular} & $\begin{array}{l}\text { Diff. of the } \\
\text { log of the } \\
\text { Size }\end{array}$ & Counts & $\begin{array}{l}\text { Weighted } \\
\text { Mean } \\
\text { Diameter }\end{array}$ \\
\hline $0.30-0.49$ & .38 & 0.21 & 146831 & \multirow{6}{*}{0.62} \\
\hline $0.50-0.99$ & .70 & 0.30 & 44446 & \\
\hline $1.00-1.99$ & 1.41 & 0.30 & 9840 & \\
\hline $2.00-4.99$ & 3.16 & 0.40 & 6746 & \\
\hline $5.00-9.99$ & 7.07 & 0.30 & 407 & \\
\hline$>10.00$ & 14.14 & 0.30 & 301 & \\
\hline
\end{tabular}

\begin{tabular}{|c|c|c|c|c|}
\hline \multicolumn{5}{|l|}{ Sample \#6 } \\
\hline $\begin{array}{l}\text { Bin } \\
\text { Range } \\
(\mu \mathrm{m})\end{array}$ & \begin{tabular}{|l} 
Bin Mean \\
Diameter \\
$(\mathrm{um})$
\end{tabular} & $\begin{array}{l}\text { Diff. of the } \\
\text { log of the } \\
\text { Size }\end{array}$ & Counts & \begin{tabular}{|l} 
Weighted \\
Mean \\
Diameter
\end{tabular} \\
\hline $0.30-0.49$ & .38 & 0.21 & 146846 & \multirow{6}{*}{0.63} \\
\hline $0.50-0.99$ & .70 & 0.30 & 44396 & \\
\hline $1.00-1.99$ & 1.41 & 0.30 & 9835 & \\
\hline $2.00-4.99$ & 3.16 & 0.40 & 6864 & \\
\hline $5.00-9.99$ & 7.07 & 0.30 & 409 & \\
\hline$>10.00$ & 14.14 & 0.30 & 432 & \\
\hline
\end{tabular}

\begin{tabular}{|c|c|c|c|c|}
\hline \multicolumn{5}{|c|}{ Sample \#8 } \\
\hline $\begin{array}{l}\text { Bin } \\
\text { Range } \\
(\mu \mathrm{m})\end{array}$ & \begin{tabular}{|l} 
Bin Mean \\
Diameter \\
$(\mathrm{lm})$
\end{tabular} & $\begin{array}{l}\text { Diff. of the } \\
\text { log of the } \\
\text { Size }\end{array}$ & $\begin{array}{l}\text { Counts } \\
\text {. }\end{array}$ & \begin{tabular}{|l} 
Weighted \\
Mean \\
Diameter
\end{tabular} \\
\hline $0.30-0.49$ & .38 & 0.21 & 142473 & \multirow{6}{*}{0.72} \\
\hline $0.50-0.99$ & .70 & 0.30 & $\overline{44092}$ & \\
\hline $0.00-1.99$ & 1.41 & 0.30 & 10255 & \\
\hline $2.00-4.99$ & 3.16 & 0.40 & 7998 & \\
\hline $5.00-9.99$ & 7.07 & 0.30 & 712 & \\
\hline$>10.00$ & 14.14 & 0.30 & 1340 & \\
\hline
\end{tabular}

\begin{tabular}{|c|c|c|c|c|}
\hline $\begin{array}{l}\text { Bin } \\
\text { Range } \\
(u m)\end{array}$ & \begin{tabular}{|l} 
Bin Mean \\
Diameter \\
(um)
\end{tabular} & $\begin{array}{l}\text { Diff. of the } \\
\text { log of the } \\
\text { Size }\end{array}$ & Counts & $\begin{array}{l}\text { Weighted } \\
\text { Mean } \\
\text { Diamoter }\end{array}$ \\
\hline $0.30-0.49$ & .38 & 0.21 & 147627 & \multirow{6}{*}{0.62} \\
\hline $0.50-0.99$ & .70 & 0.30 & 44998 & \\
\hline $1.00-1.99$ & 1.41 & 0.30 & 9871 & \\
\hline $2.00-4.99$ & 3.16 & 0.40 & 6946 & \\
\hline $5.00-9.99$ & 7.07 & 0.30 & 415 & \\
\hline$>10.00$ & 14.14 & 0.30 & 345 & \\
\hline
\end{tabular}




\section{Aerosol Particle Measurement Record}

Description of sample location 3706 Building, Room 102, Ambient Air Test, at 1.0 meter, Date 7/27/94, Time sample started 12:32:00, Temperature 78.5 F, Humidity 39.5\%, Dew Point $50.7 \mathrm{~F}$, Average Wind Velocity (if applicable) $.00 \mathrm{~m} / \mathrm{s}$, \# of physical barriers between sample area and outside (if applicable). $\underline{3}$,

\begin{tabular}{|c|c|c|c|c|}
\hline $\begin{array}{l}\text { Bin } \\
\text { Range } \\
(\mu \mathrm{m})\end{array}$ & $\begin{array}{l}\text { Bin Mean } \\
\text { Diameter } \\
(\mu \mathrm{m})\end{array}$ & $\begin{array}{l}\text { Diff. of the } \\
\log \text { of the } \\
\text { Size }\end{array}$ & Counts & $\begin{array}{l}\text { Weighted } \\
\text { Mean } \\
\text { Diameter }\end{array}$ \\
\hline $0.30-0.49$ & 0.38 & 0.21 & 129555 & \multirow{6}{*}{0.61} \\
\hline $0.50-0.99$ & 0.70 & 0.30 & 76605 & \\
\hline $1.00-1.99$ & 1.41 & 0.30 & 6730 & \\
\hline $2.00 \cdot 4.99$ & 3.16 & 0.40 & 4966 & \\
\hline $5.00-9.99$ & 7.07 & 0.30 & 302 & \\
\hline$>10.00$ & 14.14 & 0.30 & 293 & \\
\hline \multicolumn{5}{|l|}{ Sample \#3 } \\
\hline \begin{tabular}{|l} 
Bin \\
Range \\
$(u$ m)
\end{tabular} & $\begin{array}{l}\text { Bin Mean } \\
\text { Diameter } \\
(u m)\end{array}$ & $\begin{array}{l}\text { Diff. of the } \\
\text { log of the } \\
\text { Size }\end{array}$ & Counts & $\begin{array}{l}\text { Weighted } \\
\text { Mean } \\
\text { Diameter }\end{array}$ \\
\hline $0.30-0.49$ & 0.38 & 0.21 & 126078 & \multirow{6}{*}{0.61} \\
\hline $0.50-0.99$ & 0.70 & 0.30 & 74159 & \\
\hline $1.00 \cdot 1.99$ & 1.41 & 0.30 & 6473 & \\
\hline $2.00-4.99$ & 3.16 & 0.40 & 4680 & \\
\hline $5.00-9.99$ & 7.07 & 0.30 & 279 & \\
\hline$>10.00$ & 14.14 & 0.30 & 234 & \\
\hline \multicolumn{5}{|l|}{ Sample \#5 } \\
\hline $\begin{array}{l}\text { Bin } \\
\text { Range } \\
(u \mathrm{~m}) \\
\end{array}$ & $\begin{array}{l}\text { Bin Mean } \\
\text { Diameter } \\
(\mu \mathrm{m})\end{array}$ & $\begin{array}{l}\text { Diff. of the } \\
\log \text { of the } \\
\text { Size }\end{array}$ & Counts & $\begin{array}{l}\text { Weighted } \\
\text { Mean } \\
\text { Diameter }\end{array}$ \\
\hline $0.30-0.49$ & 0.38 & 0.21 & 123451 & \multirow{6}{*}{0.60} \\
\hline $0.50-0.99$ & 0.70 & 0.30 & 72755 & \\
\hline $1.00-1.99$ & 1.41 & 0.30 & 6283 & \\
\hline $2.00-4.99$ & 3.16 & 0.40 & 4416 & \\
\hline $5.00-9.99$ & 7.07 & 0.30 & 225 & \\
\hline$>10.00$ & 14.14 & 0.30 & 152 & \\
\hline
\end{tabular}

\begin{tabular}{|c|c|c|c|c|}
\hline \multicolumn{5}{|l|}{ Sample \#7 } \\
\hline $\begin{array}{l}\text { Bin } \\
\text { Range } \\
(\mu \mathrm{m})\end{array}$ & \begin{tabular}{|l|} 
Bin Mean \\
Diameter \\
$(\mu \mathrm{m})$
\end{tabular} & $\begin{array}{l}\text { Diff. of the } \\
\text { log of the } \\
\text { Size }\end{array}$ & Counts & $\begin{array}{l}\text { Weighted } \\
\text { Mean } \\
\text { Diameter }\end{array}$ \\
\hline $0.30-0.49$ & 0.38 & 0.21 & 125243 & \multirow{6}{*}{0.60} \\
\hline $0.50-0.99$ & 0.70 & 0.30 & 74056 & \\
\hline $1.00-1.99$ & 1.41 & 0.30 & 6278 & \\
\hline $2.00 \cdot 4.99$ & 3.16 & 0.40 & $\overline{4474}$ & \\
\hline $5.00-9.99$ & 7.07 & 0.30 & 237 & \\
\hline$>10.00$ & $\overline{14.14}$ & 0.30 & 142 & \\
\hline \multicolumn{5}{|l|}{ Sample \#9 } \\
\hline \begin{tabular}{|l} 
Bin \\
Range \\
$(\mu \mathrm{m})$
\end{tabular} & \begin{tabular}{|l} 
Bin Mean \\
Diameter \\
$(u \mathrm{~m})$
\end{tabular} & $\begin{array}{l}\text { Diff. of the } \\
\text { log of the } \\
\text { Sizie }\end{array}$ & Counts & $\begin{array}{l}\text { Weighted } \\
\text { Mean } \\
\text { Diameter }\end{array}$ \\
\hline $0.30-0.49$ & 0.38 & 0.21 & 126107 & \multirow{6}{*}{0.62} \\
\hline $0.50 \cdot 0.99$ & 0.70 & 0.30 & 76578 & \\
\hline $1.00-1.99$ & 1.41 & 0.30 & 6649 & \\
\hline $2.00-4.99$ & 3.16 & 0.40 & 5007 & \\
\hline $5.00-9.99$ & 7.07 & 0.30 & 323 & \\
\hline$>10.00$ & 14.14 & 0.30 & 326 & \\
\hline
\end{tabular}

Sample \#2

\begin{tabular}{|c|c|c|c|c|}
\hline $\begin{array}{l}\text { Bin } \\
\text { Range } \\
(u m)\end{array}$ & \begin{tabular}{|l} 
Bin Mean \\
Diameter \\
$(\mathrm{um})$
\end{tabular} & $\begin{array}{l}\text { Diff. of the } \\
\log \text { of the } \\
\text { Size }\end{array}$ & Counts & $\begin{array}{l}\text { Weighted } \\
\text { Mean } \\
\text { Diameter }\end{array}$ \\
\hline $0.30-0.49$ & .38 & 0.21 & 123278 & \multirow{6}{*}{0.63} \\
\hline $0.50-0.99$ & .70 & 0.30 & 74696 & \\
\hline $1.00-1.99$ & 1.41 & 0.30 & 6727 & \\
\hline $2.00-4.99$ & 3.16 & 0.40 & 5037 & \\
\hline $5.00-9.99$ & 7.07 & 0.30 & 327 & \\
\hline$>10.00$ & 14.14 & 0.30 & 341 & \\
\hline
\end{tabular}

\begin{tabular}{|c|c|c|c|c|}
\hline \multicolumn{5}{|l|}{ Sample \#4 } \\
\hline $\begin{array}{l}\text { Bin } \\
\text { Range } \\
(\mu \mathrm{m})\end{array}$ & \begin{tabular}{|l} 
Bin Mean \\
Diameter \\
$(\mu \mathrm{m})$
\end{tabular} & $\begin{array}{l}\text { Diff. of the } \\
\text { log of the } \\
\text { Size }\end{array}$ & Counts & $\begin{array}{l}\text { Weighted } \\
\text { Mean } \\
\text { Diameter }\end{array}$ \\
\hline $0.30-0.49$ & .38 & 0.21 & 124158 & \multirow{6}{*}{0.61} \\
\hline $0.50-0.99$ & .70 & 0.30 & 73662 & \\
\hline $1.00-1.99$ & 1.41 & 0.30 & 6393 & \\
\hline $2.00-4.99$ & 3.16 & 0.40 & $\overline{4660}$ & \\
\hline $5.00-9.99$ & 7.07 & 0.30 & 254 & \\
\hline$>10.00$ & 14.14 & 0.30 & $\overline{201}$ & \\
\hline \multicolumn{5}{|l|}{ Sample \#6 } \\
\hline $\begin{array}{l}\text { Bin } \\
\text { Range } \\
(u m)\end{array}$ & \begin{tabular}{|l} 
Bin Mean \\
Diameter \\
$(u m)$
\end{tabular} & $\begin{array}{l}\text { Diff. of the } \\
\text { log of the } \\
\text { Size }\end{array}$ & Counts & $\begin{array}{l}\text { Weighted } \\
\text { Mean } \\
\text { Diameter }\end{array}$ \\
\hline $0.30-0.49$ & .38 & 0.21 & 125102 & \multirow{6}{*}{0.62} \\
\hline $0.50-0.99$ & .70 & 0.30 & 75167 & \\
\hline $1.00-1.99$ & 1.41 & 0.30 & 6383 & \\
\hline $2.00-4.99$ & 3.16 & 0.40 & 4740 & \\
\hline $5.00-9.99$ & 7.07 & 0.30 & $\overline{316}$ & \\
\hline$>10.00$ & 14.14 & 0.30 & 335 & \\
\hline
\end{tabular}

\begin{tabular}{|c|c|c|c|c|}
\hline \multicolumn{5}{|c|}{ Sample \#8 } \\
\hline $\begin{array}{l}\text { Bin } \\
\text { Range } \\
(\mathrm{um})\end{array}$ & $\begin{array}{l}\text { Bin Mean } \\
\text { Diameter } \\
(u m)\end{array}$ & $\begin{array}{l}\text { Diff. of the } \\
\text { log of the } \\
\text { Size }\end{array}$ & Counts & $\begin{array}{l}\text { Weighted } \\
\text { Mean } \\
\text { Diameter }\end{array}$ \\
\hline $0.30-0.49$ & .38 & 0.21 & 125350 & \multirow{6}{*}{0.69} \\
\hline $0.50-0.99$ & .70 & 0.30 & 76869 & \\
\hline $0.00-1.99$ & 1.41 & 0.30 & 7011 & \\
\hline $2.00-4.99$ & 3.16 & 0.40 & 5939 & \\
\hline $5.00-9.99$ & 7.07 & 0.30 & 530 & \\
\hline$>10.00$ & 14.14 & 0.30 & 1112 & \\
\hline
\end{tabular}

\begin{tabular}{|c|c|c|c|c|}
\hline \multicolumn{5}{|c|}{ Sample \#10 } \\
\hline \begin{tabular}{|l} 
Bin \\
Range \\
(um)
\end{tabular} & $\begin{array}{l}\text { Bin Mean } \\
\text { Diameter } \\
(u \mathrm{~m})\end{array}$ & $\begin{array}{l}\text { Diff. of the } \\
\text { log of the } \\
\text { Size }\end{array}$ & Counts & $\begin{array}{l}\text { Weighted } \\
\text { Mean } \\
\text { Diameter }\end{array}$ \\
\hline $0.30-0.49$ & .38 & 0.21 & 128357 & \multirow{6}{*}{0.61} \\
\hline $0.50-0.99$ & .70 & 0.30 & 76778 & \\
\hline $1.00-1.99$ & 1.41 & 0.30 & 6468 & \\
\hline $2.00-4.99$ & 3.16 & 0.40 & 4898 & \\
\hline $5.00-9.99$ & 7.07 & 0.30 & 262 & \\
\hline$>10.00$ & 14.14 & 0.30 & 219 & \\
\hline
\end{tabular}




\section{Aerosol Particle Measurement Record}

Description of sample location 3706 Building, Room 102, Ambient Air Test, at 1.5 meters, Date 7/27/94, Time sample started 11:36:00, Temperature 76.0 F, Humidity 40.1\%, Dew Point $50.0 \mathrm{~F}$, Average Wind Velocity (if applicable) $.00 \mathrm{~m} / \mathrm{s}$, \# of physical barriers between sample area and outside (if applicable). 3 ,

Sample \#1

\begin{tabular}{|c|c|c|c|c|}
\hline $\begin{array}{l}\text { Bin } \\
\text { Range } \\
\text { (um) }\end{array}$ & $\begin{array}{l}\text { Bin Mean } \\
\text { Diameter } \\
(\mathrm{em})\end{array}$ & $\begin{array}{l}\text { Diff. of the } \\
\text { log of the } \\
\text { Size }\end{array}$ & Counts & $\begin{array}{l}\text { Weighted } \\
\text { Mean } \\
\text { Diameter }\end{array}$ \\
\hline $0.30-0.49$ & 0.38 & $0 . \overline{21}$ & 126046 & \multirow{6}{*}{0.61} \\
\hline $0.50 \cdot 0.99$ & 0.70 & 0.30 & 78013 & \\
\hline $1.00-1.99$ & 1.41 & 0.30 & 6793 & \\
\hline $2.00 \cdot 4.99$ & 3.16 & 0.40 & 4695 & \\
\hline $5.00-9.99$ & 7.07 & 0.30 & 282 & \\
\hline$>10.00$ & 14.14 & 0.30 & 229 & \\
\hline \multicolumn{5}{|l|}{ Sample \#3 } \\
\hline \begin{tabular}{|l} 
Bin \\
Range \\
$(\mu \mathrm{m})$
\end{tabular} & \begin{tabular}{|l} 
Bin Mean \\
Diameter \\
$(u m)$
\end{tabular} & $\begin{array}{l}\text { Diff. of the } \\
\log \text { of the } \\
\text { Size }\end{array}$ & Counts & $\begin{array}{l}\text { Weighted } \\
\text { Mean } \\
\text { Diameter }\end{array}$ \\
\hline $0.30-0.49$ & 0.38 & 0.21 & 125139 & \multirow{6}{*}{0.63} \\
\hline $0.50-0.99$ & 0.70 & 0.30 & 77142 & \\
\hline $1.00-1.99$ & 1.41 & 0.30 & 7113 & \\
\hline $2.00-4.99$ & 3.16 & 0.40 & 5271 & \\
\hline $5.00-9.99$ & 7.07 & 0.30 & 320 & \\
\hline$>10.00$ & 14.14 & 0.30 & 363 & \\
\hline \multicolumn{5}{|l|}{ Sample \#5 } \\
\hline $\begin{array}{l}\text { Bin } \\
\text { Range } \\
(\mu \mathrm{m})\end{array}$ & $\begin{array}{l}\text { Bin Mean } \\
\text { Diameter } \\
(u m)\end{array}$ & $\begin{array}{l}\text { Diff. of the } \\
\text { log of the } \\
\text { Size }\end{array}$ & Counts & $\begin{array}{l}\text { Weighted } \\
\text { Mean } \\
\text { Diameter }\end{array}$ \\
\hline $0.30 \cdot 0.49$ & 0.38 & 0.21 & 130648 & \multirow{6}{*}{0.61} \\
\hline $0.50 \cdot 0.99$ & 0.70 & 0.30 & 80145 & \\
\hline $1.00-1.99$ & 1.41 & 0.30 & 6880 & \\
\hline $2.00 \cdot 4.99$ & 3.16 & 0.40 & 4772 & \\
\hline $5.00 \cdot 9.99$ & 7.07 & 0.30 & 259 & \\
\hline$>10.00$ & 14.14 & 0.30 & 188 & \\
\hline \multicolumn{5}{|l|}{ Sample \#7 } \\
\hline $\begin{array}{l}\text { Bin } \\
\text { Range } \\
(\mu \mathrm{m}) \\
\end{array}$ & $\begin{array}{l}\text { Bin Mean } \\
\text { Diameter } \\
(\mu \mathrm{m}) \\
\end{array}$ & $\begin{array}{l}\text { Diff. of the } \\
\log \text { of the } \\
\text { Size }\end{array}$ & Counts & $\begin{array}{l}\text { Weighted } \\
\text { Mean } \\
\text { Diameter }\end{array}$ \\
\hline $0.30-0.49$ & 0.38 & 0.21 & 129149 & \multirow{6}{*}{0.60} \\
\hline $0.50-0.99$ & 0.70 & 0.30 & 79479 & \\
\hline $1.00-1.99$ & 1.41 & 0.30 & 6680 & \\
\hline $2.00-4.99$ & 3.16 & 0.40 & 4551 & \\
\hline $5.00-9.99$ & 7.07 & 0.30 & 215 & \\
\hline$>10.00$ & 14.14 & 0.30 & 101 & \\
\hline \multicolumn{5}{|l|}{ Sample \#9 } \\
\hline $\begin{array}{l}\text { Bin } \\
\text { Range } \\
(\mu \mathrm{m})\end{array}$ & \begin{tabular}{|l} 
Bin Mean \\
Diameter \\
$($ (um)
\end{tabular} & $\begin{array}{l}\text { Diff. of the } \\
\text { log of the } \\
\text { Size }\end{array}$ & Counts & $\begin{array}{l}\text { Weighted } \\
\text { Mean } \\
\text { Diameter }\end{array}$ \\
\hline $0.30-0.49$ & 0.38 & 0.21 & 127535 & \multirow{6}{*}{0.61} \\
\hline $0.50-0.99$ & 0.70 & 0.30 & 78027 & \\
\hline $1.00 \cdot 1.99$ & 1.41 & 0.30 & 6827 & \\
\hline $2.00-4.99$ & 3.16 & 0.40 & 4949 & \\
\hline $5.00-9.99$ & 7.07 & 0.30 & 229 & \\
\hline$>10.00$ & 14.14 & 0.30 & 226 & \\
\hline
\end{tabular}

Sample \#2

\begin{tabular}{|c|c|c|c|c|}
\hline $\begin{array}{l}\text { Bin } \\
\text { Range } \\
\text { (um) }\end{array}$ & $\begin{array}{l}\text { Bin Mean } \\
\text { Diameter } \\
(u m)\end{array}$ & $\begin{array}{l}\text { Diff. of the } \\
\text { log of the } \\
\text { Size }\end{array}$ & Counts & $\begin{array}{l}\text { Weighted } \\
\text { Mean } \\
\text { Diameter }\end{array}$ \\
\hline $0.30-0.49$ & .38 & 0.21 & 125891 & \multirow{6}{*}{0.62} \\
\hline $0.50-0.99$ & .70 & 0.30 & 77298 & \\
\hline $1.00-1.99$ & 1.41 & 0.30 & 6808 & \\
\hline $2.00-4.99$ & 3.16 & 0.40 & 4833 & \\
\hline $5.00-9.99$ & 7.07 & 0.30 & 304 & \\
\hline$>10.00$ & 14.14 & 0.30 & 318 & \\
\hline
\end{tabular}

Sample \#4

\begin{tabular}{|c|c|c|c|c|}
\hline $\begin{array}{l}\text { Bin } \\
\text { Range } \\
(\mu \mathrm{m})\end{array}$ & $\begin{array}{l}\text { Bin Mean } \\
\text { Diameter } \\
(\mu \mathrm{m})\end{array}$ & $\begin{array}{l}\text { Diff. of the } \\
\text { log of the } \\
\text { Size }\end{array}$ & Counts & $\begin{array}{l}\text { Weighted } \\
\text { Mean } \\
\text { Diameter }\end{array}$ \\
\hline $0.30-0.49$ & .38 & 0.21 & 124550 & \multirow{6}{*}{0.63} \\
\hline $0.50-0.99$ & .70 & 0.30 & 77306 & \\
\hline $1.00-1.99$ & 1.41 & 0.30 & 7015 & \\
\hline $2.00-4.99$ & 3.16 & 0.40 & 5131 & \\
\hline $5.00-9.99$ & 7.07 & 0.30 & 362 & \\
\hline$>10.00$ & 14.14 & 0.30 & 302 & \\
\hline
\end{tabular}

\begin{tabular}{|c|c|c|c|c|}
\hline \multicolumn{5}{|l|}{ Sample \#6 } \\
\hline $\begin{array}{l}\text { Bin } \\
\text { Range } \\
(\mu \mathrm{m})\end{array}$ & \begin{tabular}{|l|} 
Bin Mean \\
Diameter \\
$(u m)$
\end{tabular} & $\begin{array}{l}\text { Diff. of the } \\
\text { log of the } \\
\text { Size }\end{array}$ & Counts & $\begin{array}{l}\text { Weighted } \\
\text { Mean } \\
\text { Diameter }\end{array}$ \\
\hline $0.30-0.49$ & .38 & 0.21 & 128038 & \multirow{6}{*}{0.60} \\
\hline $0.50-0.99$ & .70 & 0.30 & 78412 & \\
\hline $1.00-1.99$ & 1.41 & 0.30 & 6768 & \\
\hline $2.00-4.99$ & 3.16 & 0.40 & 4514 & \\
\hline $5.00-9.99$ & 7.07 & 0.30 & 206 & \\
\hline$>10.00$ & 14.14 & 0.30 & 108 & \\
\hline
\end{tabular}

\section{Sample \#8}

\begin{tabular}{|c|c|c|c|c|}
\hline $\begin{array}{l}\text { Bin } \\
\text { Range } \\
\text { (um) }\end{array}$ & \begin{tabular}{|l|} 
Bin Mean \\
Diameter \\
(um)
\end{tabular} & $\begin{array}{l}\text { Diff. of the } \\
\text { log of the } \\
\text { Size }\end{array}$ & Counts & $\begin{array}{l}\text { Weighted } \\
\text { Mean } \\
\text { Diameter }\end{array}$ \\
\hline $0.30-0.49$ & .38 & 0.21 & 128597 & \multirow{6}{*}{0.60} \\
\hline $0.50-0.99$ & .70 & 0.30 & 78562 & \\
\hline $0.00-1.99$ & 1.41 & 0.30 & 6848 & \\
\hline $2.00-4.99$ & 3.16 & 0.40 & 4728 & \\
\hline $5.00-9.99$ & 7.07 & 0.30 & 241 & \\
\hline$>10.00$ & 14.14 & 0.30 & 169 & \\
\hline
\end{tabular}
Sample \#10

\begin{tabular}{|c|c|c|c|c|}
\hline $\begin{array}{l}\text { Bin } \\
\text { Range } \\
(u m)\end{array}$ & \begin{tabular}{|l|} 
Bin Mean \\
Diameter \\
$(\mu \mathrm{m})$
\end{tabular} & $\begin{array}{l}\text { Diff, of the } \\
\text { log of the } \\
\text { Size }\end{array}$ & Counts & $\begin{array}{l}\text { Weighted } \\
\text { Mean } \\
\text { Diameter }\end{array}$ \\
\hline $0.30-0.49$ & .38 & 0.21 & 127223 & \multirow{6}{*}{0.61} \\
\hline $0.50-0.99$ & .70 & 0.30 & 78538 & \\
\hline $1.00-1.99$ & 1.41 & 0.30 & 7030 & \\
\hline $2.00-4.99$ & 3.16 & 0.40 & 4841 & \\
\hline $5.00-9.99$ & 7.07 & 0.30 & 273 & \\
\hline$>10.00$ & 14.14 & 0.30 & 181 & \\
\hline
\end{tabular}




\section{Aerosol Particle Measurement Record}

Description of sample location 3706 Building, Room 102, Ambient Air Test, at 2.0 meters, Date 7/27/94, Time sample started 13:39, Temperature 82.2 F, Humidity 37.6 \%, Dew Point 53.6 F. Average Wind Velocity (if applicable) $.00 \mathrm{~m} / \mathrm{s}$, \# of physical barriers between sample area and outside (if applicable). 3 ,

Sample \#1

\begin{tabular}{|c|c|c|c|c|}
\hline $\begin{array}{l}\text { Bin } \\
\text { Range } \\
\text { (um) }\end{array}$ & $\begin{array}{l}\text { Bin Mean } \\
\text { Diameter } \\
\text { (um) }\end{array}$ & $\begin{array}{l}\text { Diff. of the } \\
\log \text { of the } \\
\text { Size }\end{array}$ & Counts & $\begin{array}{l}\text { Weighted } \\
\text { Mean } \\
\text { Diameter }\end{array}$ \\
\hline $0.30-0.49$ & 0.38 & 0.21 & 125689 & \multirow{6}{*}{0.63} \\
\hline $0.50 \cdot 0.99$ & 0.70 & 0.30 & 75514 & \\
\hline $1.00-1.99$ & 1.41 & 0.30 & 6544 & \\
\hline $2.00-4.99$ & 3.16 & 0.40 & 4421 & \\
\hline $5.00-9.99$ & 7.07 & 0.30 & 327 & \\
\hline$>10.00$ & 14.14 & 0.30 & 539 & \\
\hline
\end{tabular}

Sample \#3

\begin{tabular}{||c|c|c|r|r||}
\hline $\begin{array}{l}\text { Bin } \\
\text { Range } \\
(\mu \mathrm{m})\end{array}$ & $\begin{array}{l}\text { Bin Mean } \\
\text { Diameter } \\
(\mu \mathrm{m})\end{array}$ & $\begin{array}{l}\text { Diff. of the } \\
\text { log of the } \\
\text { Size }\end{array}$ & Counts & $\begin{array}{l}\text { Weighted } \\
\text { Mean } \\
\text { Diameter }\end{array}$ \\
\hline $0.30-0.49$ & 0.38 & 0.21 & 125942 & \\
\cline { 1 - 4 } $0.50-0.99$ & 0.70 & 0.30 & 75429 & \multirow{2}{*}{0.60} \\
\hline $1.00-1.99$ & 1.41 & 0.30 & 6194 & \\
\hline $2.00-4.99$ & 3.16 & 0.40 & 4014 & \\
\cline { 1 - 4 } $5.00-9.99$ & 7.07 & 0.30 & 232 & \\
\hline$>10.00$ & 14.14 & 0.30 & 228 & \\
\hline
\end{tabular}

Sample \#5

\begin{tabular}{|c|c|c|c|c|}
\hline $\begin{array}{l}\text { Bin } \\
\text { Range } \\
(\mu \mathrm{m})\end{array}$ & $\begin{array}{l}\text { Bin Mean } \\
\text { Diameter } \\
(\mu \mathrm{m})\end{array}$ & $\begin{array}{l}\text { Diff. of the } \\
\log \text { of the } \\
\text { Size }\end{array}$ & Counts & $\begin{array}{l}\text { Weighted } \\
\text { Mean } \\
\text { Diameter }\end{array}$ \\
\hline $0.30 \cdot 0.49$ & 0.38 & 0.21 & 136207 & \multirow{6}{*}{0.58} \\
\hline $0.50 \cdot 0.99$ & 0.70 & 0.30 & 79241 & \\
\hline $1.00-1.99$ & 1.41 & 0.30 & 6028 & \\
\hline $2.00-4.99$ & 3.16 & 0.40 & 3723 & \\
\hline $5.00 \cdot 9.99$ & 7.07 & 0.30 & $\overline{204}$ & \\
\hline$>10.00$ & 14.14 & 0.30 & $\overline{172}$ & \\
\hline
\end{tabular}

Sample \#7

\begin{tabular}{|c|c|c|c|c|}
\hline \begin{tabular}{|l|} 
Bin \\
Range \\
$(\mu \mathrm{m})$ \\
\end{tabular} & \begin{tabular}{|l|} 
Bin Mean \\
Diameter \\
$(\mu \mathrm{m})$
\end{tabular} & $\begin{array}{l}\text { Diff. of the } \\
\log \text { of the } \\
\text { Size }\end{array}$ & Counts & $\begin{array}{l}\text { Weighted } \\
\text { Mean } \\
\text { Diameter }\end{array}$ \\
\hline $0.30-0.49$ & 0.38 & 0.21 & 126084 & \multirow{6}{*}{0.59} \\
\hline $0.50-0.99$ & 0.70 & 0.30 & 74116 & \\
\hline $1.00-1.99$ & 1.41 & 0.30 & 5874 & \\
\hline $2.00-4.99$ & 3.16 & 0.40 & 3645 & \\
\hline $5.00-9.99$ & 7.07 & 0.30 & 168 & \\
\hline$>10.00$ & 14.14 & 0.30 & 166 & \\
\hline \multicolumn{5}{|l|}{ Sample \#9 } \\
\hline $\begin{array}{l}\text { Bin } \\
\text { Range } \\
(\mu \mathrm{m}) \\
\end{array}$ & \begin{tabular}{|l|} 
Bin Mean \\
Diameter \\
$(\mathrm{\mu m})$
\end{tabular} & $\begin{array}{l}\text { Diff. of the } \\
\text { log of the } \\
\text { Size }\end{array}$ & Counts & $\begin{array}{l}\text { Weighted } \\
\text { Mean } \\
\text { Diameter }\end{array}$ \\
\hline $0.30-0.49$ & 0.38 & 0.21 & 128047 & \multirow{6}{*}{$\begin{array}{l}9.59 \\
3 \\
3 \\
3\end{array}$} \\
\hline 0.50 .0 .99 & 0.70 & 0.30 & 75189 & \\
\hline $1.00-1.99$ & 1.41 & 0.30 & 5693 & \\
\hline $2.00-4.99$ & 3.16 & 0.40 & 3609 & \\
\hline $5.00-9.99$ & 7.07 & 0.30 & 191 & \\
\hline$>10.00$ & 14.14 & 0.30 & 249 & \\
\hline
\end{tabular}

Sample \#2

\begin{tabular}{||l|c|c|r|r||}
\hline $\begin{array}{l}\text { Rin } \\
\text { Range } \\
(\mu \mathrm{m})\end{array}$ & $\begin{array}{l}\text { Bin Mean } \\
\text { Diameter } \\
(\mathrm{mm})\end{array}$ & $\begin{array}{l}\text { Diff. of the } \\
\text { log of the } \\
\text { Size }\end{array}$ & Counts & $\begin{array}{l}\text { Weighted } \\
\text { Mean } \\
\text { Diameter }\end{array}$ \\
\hline $0.30-0.49$ & .38 & 0.21 & 124909 & \\
\cline { 1 - 4 } $0.50-0.99$ & .70 & 0.30 & 75201 & \multirow{2}{*}{0.61} \\
\hline $1.00-1.99$ & 1.41 & 0.30 & 6444 & \\
\hline $2.00-4.99$ & 3.16 & 0.40 & 4139 & \\
\hline $5.00-9.99$ & 7.07 & 0.30 & 234 & \\
\hline$>10.00$ & 14.14 & 0.30 & 395 &. \\
\hline
\end{tabular}

\begin{tabular}{|c|c|c|c|c|}
\hline \multicolumn{5}{|c|}{ Sample \#4 } \\
\hline $\begin{array}{l}\text { Bin } \\
\text { Range } \\
\text { (um) }\end{array}$ & \begin{tabular}{|l} 
Bin Mean \\
Diameter \\
$(\mathrm{erm})$
\end{tabular} & $\begin{array}{l}\text { Diff. of the } \\
\text { log of the } \\
\text { Size }\end{array}$ & Counts & $\begin{array}{l}\text { Weighted } \\
\text { Mean } \\
\text { Diameter }\end{array}$ \\
\hline $0.30-0.49$ & .38 & 0.21 & 126196 & \multirow{6}{*}{0.59} \\
\hline $0.50-0.99$ & .70 & 0.30 & 75344 & \\
\hline $1.00-1.99$ & 1.41 & 0.30 & 6042 & \\
\hline $2.00-4.99$ & 3.16 & 0.40 & 3877 & \\
\hline $5.00-9.99$ & 7.07 & 0.30 & 214 & \\
\hline$>10.00$ & 14.14 & 0.30 & 202 & \\
\hline
\end{tabular}

\begin{tabular}{|c|c|c|c|c|}
\hline \multicolumn{5}{|c|}{ Sample $\# 6$} \\
\hline \begin{tabular}{|l} 
Bin \\
Range \\
$(\mu \mathrm{m})$
\end{tabular} & \begin{tabular}{|l} 
Bin Mean \\
Diameter \\
$(u m)$
\end{tabular} & $\begin{array}{l}\text { Diff. of the } \\
\log \text { of the } \\
\text { Size }\end{array}$ & Counts & $\begin{array}{l}\text { Weighted } \\
\text { Mean } \\
\text { Diameter }\end{array}$ \\
\hline $0.30-0.49$ & .38 & 0.21 & 126566 & \multirow{6}{*}{0.59} \\
\hline $0.50-0.99$ & .70 & 0.30 & 74820 & \\
\hline $1.00-1.99$ & 1.41 & 0.30 . & 6032 & \\
\hline $2.00-4.99$ & 3.16 & 0.40 & 3808 & \\
\hline $5.00-9.99$ & 7.07 & 0.30 & 191 & \\
\hline$>10.00$ & 14.14 & 0.30 & 230 & \\
\hline
\end{tabular}

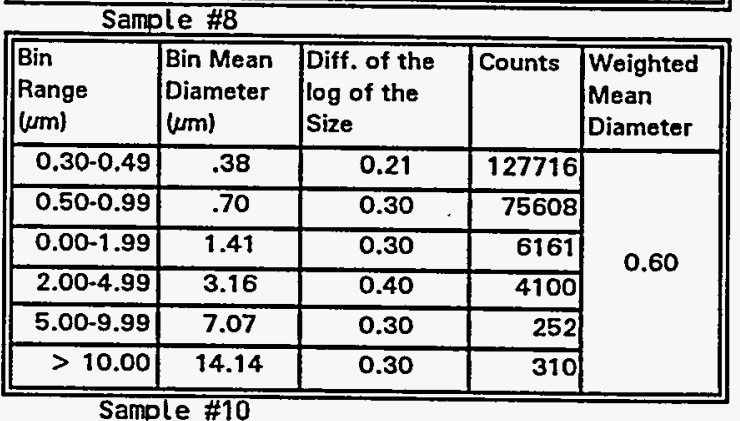

\begin{tabular}{|c|c|c|c|c|}
\hline \begin{tabular}{|l} 
Bin \\
Range \\
$(\mu / m)$
\end{tabular} & \begin{tabular}{|l} 
Bin Mean \\
Diameter \\
$(\mathrm{um})$
\end{tabular} & $\begin{array}{l}\text { Diff. of the } \\
\text { log of the } \\
\text { Size }\end{array}$ & Counts & $\begin{array}{l}\text { Weighted } \\
\text { Mean } \\
\text { Diameter }\end{array}$ \\
\hline $0.30-0.49$ & .38 & 0.21 & 127587 & \multirow{6}{*}{0.58} \\
\hline $0.50-0.99$ & .70 & 0.30 & 73536 & \\
\hline $1.00-1.99$ & 1.41 & 0.30 & 5696 & \\
\hline $2.00-4.99$ & 3.16 & 0.40 & 3479 & \\
\hline $5.00-9.99$ & 7.07 & 0.30 & 146 & \\
\hline$>10.00$ & 14.14 & 0.30 & 129 & \\
\hline
\end{tabular}


Data for Table 5. Mean Optical Diameter of Ambient Air in PUREX

$2 \mathrm{~cm} .(0.02$ meters)

0.5 meters

1.0 meters

1.5 meters

2.0 meters 


\section{Aerosol Particle Measurement Record}

Description of sample location PUREX P\&O Gallery, Ambient Air Test, at $2 \mathrm{~cm}$,

Date_9/07/94, Time sample started_9:27:46. Temperature_82.2 F, Humidity $34.6 \%$, Dew Point $51.6 \mathrm{~F}$, Average Wind Velocity (if applicable) $.00 \mathrm{~m} / \mathrm{s}$, \# of physical barriers between sample area and outside (if applicable). 3 ,

Sample \#1
\begin{tabular}{||c|c|c|r|r||}
\hline \begin{tabular}{|l|l|r|} 
Bin \\
Range \\
$(\mu \mathrm{m})$
\end{tabular} & $\begin{array}{l}\text { Bin Mean } \\
\text { Diameter } \\
(\mu \mathrm{m})\end{array}$ & $\begin{array}{l}\text { Diff. of the } \\
\text { log of the } \\
\text { Size }\end{array}$ & Counts & $\begin{array}{l}\text { Weighted } \\
\text { Mean } \\
\text { Diameter }\end{array}$ \\
\hline $0.30-0.49$ & 0.38 & 0.21 & 47824 & \\
\hline $0.50-0.99$ & 0.70 & 0.30 & 12831 & \\
\hline $1.00-1.99$ & 1.41 & 0.30 & 3812 & \\
\hline $2.00-4.99$ & 3.16 & 0.40 & 3663 & \\
\hline $5.00-9.99$ & 7.07 & 0.30 & 294 & \\
\hline$>10.00$ & 14.14 & 0.30 & 434 & \\
\hline
\end{tabular}




\section{Aerosol Particle Measurement Record}

Description of sample location PUREX P\&O Gallery, Ambient Air Test, at $0.5 \mathrm{~m}$,

Date_9/07/94, Time sample started 9:52:20, Temperature 82.7 F, Humidity $33.7 \%$,

Dew Point $51.2 \mathrm{~F}$, Average Wind Velocity (if applicable) $.00 \mathrm{~m} / \mathrm{s}$, \# of physical barriers between sample area and outside (if applicable). 3 ,

Sample \#1
\begin{tabular}{||c|c|c|r|r||}
\hline \begin{tabular}{||l|l|r||} 
Bin \\
Range \\
$\mu \mathrm{m})$
\end{tabular} & $\begin{array}{l}\text { Bin Mean } \\
\text { Diameter } \\
(\mu \mathrm{m})\end{array}$ & $\begin{array}{l}\text { Diff. of the } \\
\text { log of the } \\
\text { Size }\end{array}$ & Counts & $\begin{array}{l}\text { Weighted } \\
\text { Mean } \\
\text { Diameter }\end{array}$ \\
\hline $0.30-0.49$ & 0.38 & 0.21 & 46464 & \\
\hline $0.50-0.99$ & 0.70 & 0.30 & 11774 & \\
\hline $1.00-1.99$ & 1.41 & 0.30 & 3140 & \\
\hline $2.00-4.99$ & 3.16 & 0.40 & 2641 & \\
\hline $5.00-9.99$ & 7.07 & 0.30 & 154 & \\
\hline$>10.00$ & 14.14 & 0.30 & 117 & \\
\hline
\end{tabular}




\section{Aerosol Particle Measurement Record}

Description of sample location PUREX P\&O Gallery, Ambient Air Test, at $1.0 \mathrm{~m}$,

Date_9/07/94, Time sample started 9:52:20, Temperature 82.7 F, Humidity $33.7 \%$, Dew Point $51.2 \mathrm{~F}$, Average Wind Velocity (if applicable) $.00 \mathrm{~m} / \mathrm{s}$, \# of physical barriers between sample area and outside (if applicable). $\underline{3}$,

Sample \#1
\begin{tabular}{||c|c|c|r|r||}
\hline $\begin{array}{l}\text { Bin } \\
\text { Range } \\
(\mu \mathrm{m})\end{array}$ & $\begin{array}{l}\text { Bin Mean } \\
\text { Diameter } \\
(\mu \mathrm{m})\end{array}$ & $\begin{array}{l}\text { Diff. of the } \\
\text { log of the } \\
\text { Size }\end{array}$ & Counts & $\begin{array}{l}\text { Weighted } \\
\begin{array}{l}\text { Mean } \\
\text { Diameter }\end{array}\end{array}$ \\
\hline $0.30-0.49$ & 0.38 & 0.21 & 48586 & \\
\hline $0.50-0.99$ & 0.70 & 0.30 & 28638 \\
\hline $1.00-1.99$ & 1.41 & 0.30 & 3072 & 0.65 \\
\hline $2.00-4.99$ & 3.16 & 0.40 & 2596 & \\
\hline $5.00-9.99$ & 7.07 & 0.30 & 186 & \\
\hline$>10.00$ & 14.14 & 0.30 & 97 & \\
\hline
\end{tabular}




\section{Aerosol Particle Measurement Record}

Description of sample location PUREX P\&O Gallerv, Ambient Air Test, at $1.5 \mathrm{~m}$, Date 9/07/94, Time sample started_9:36:44, Temperature 83.5 F, Humidity $33.3 \%$, Dew Point 50.8 F, Average Wind Velocity (if applicable) $.00 \mathrm{~m} / \mathrm{s}$, \# of physical barriers between sample area and outside (if applicable). $\underline{3}$,

\begin{tabular}{|c|c|c|c|c|}
\hline $\begin{array}{l}\text { Bin } \\
\text { Range } \\
\text { (um) }\end{array}$ & \begin{tabular}{|l|} 
Bin Mean \\
Diameter \\
$(\mathrm{um})$
\end{tabular} & $\begin{array}{l}\text { Diff. of the } \\
\text { log of the } \\
\text { Size }\end{array}$ & Counts & \begin{tabular}{|l|} 
Weighted \\
Mean \\
Diameter
\end{tabular} \\
\hline $0.30-0.49$ & 0.38 & 0.21 & 48304 & \multirow{6}{*}{0.63} \\
\hline $0.50-0.99$ & 0.70 & 0.30 & 29035 & \\
\hline $1.00-1.99$ & 1.41 & 0.30 & 3053 & \\
\hline $2.00-4.99$ & 3.16 & 0.40 & 2571 & \\
\hline $5.00-9.99$ & 7.07 & 0.30 & 158 & \\
\hline$>10.00$ & 14.14 & 0.30 & 31 & \\
\hline
\end{tabular}




\section{Aerosol Particle Measurement Record}

Description of sample location PUREX P\&O Gallery. Ambient Air Test, at $2.0 \mathrm{~m}$,

Date 9/07/94. Time sample started 9:27:46, Temperature 82.2 F, Humidity 34.6\%, Dew Point $51.6 \mathrm{~F}$, Average Wind Velocity (if applicable) $.00 \mathrm{~m} / \mathrm{s}$, \# of physical barriers between sample area and outside (if applicable). $\underline{3}$,

Sample \#1
\begin{tabular}{||c|c|c|r|r||}
\hline \begin{tabular}{|l|l|r||} 
Bin \\
Range \\
$(u \mathrm{~m})$
\end{tabular} & $\begin{array}{l}\text { Bin Mean } \\
\text { Diameter } \\
(u m)\end{array}$ & $\begin{array}{l}\text { Diff. of the } \\
\text { log of the } \\
\text { Size }\end{array}$ & Counts & $\begin{array}{l}\text { Weighted } \\
\text { Mean } \\
\text { Diameter }\end{array}$ \\
\hline $0.30-0.49$ & 0.38 & 0.21 & 50000 & \\
\hline $0.50-0.99$ & 0.70 & 0.30 & 28905 & \\
\hline $1.00-1.99$ & 1.41 & 0.30 & 2857 & \\
\hline $2.00-4.99$ & 3.16 & 0.40 & 2371 & \\
\hline $5.00-9.99$ & 7.07 & 0.30 & 123 & \\
\hline$>10.00$ & 14.14 & 0.30 & 56 & \\
\hline
\end{tabular}


Data for Fig 2. Mean Optical Diameter Versus Time,

Building 3706A,

Fig. 3 Resuspension and Ambient Air Vs. Height, Building

$3706 \mathrm{~A}$

Table 6. Peak Mean Diameter ( $\mu \mathrm{m})$, Versus Sampling Height,

and

Table 7. Percent of Large Particles in Breathing Zone, Building 3706A

$0.5 \mathrm{~m}$

$1.0 \mathrm{~m}$

$1.5 \mathrm{~m}$

$2.0 \mathrm{~m}$ with

$0.25 \mathrm{~m}$

$0.25 \mathrm{~m}$

with

with

$0.25 \mathrm{~m}$

$0.25 \mathrm{~m}$

with 


\section{Aerosol Particle Measurement Record}

Description of sample location 3706A Building, Resuspension Test, at 0.5, Date_8/12/94, Time sample started 10:49:54, Temperature 82.0 F, Humidity $38.2 \%$, Dew Point 53.9 F ; Average Wind Velocity (if applicable) $.00 \mathrm{~m} / \mathrm{s}$, \# of physical barriers between sample area and outside (if applicable) 1

Sample \#1

\begin{tabular}{|c|c|c|c|c|}
\hline $\begin{array}{l}\text { Bin } \\
\text { Range } \\
\text { (um) }\end{array}$ & $\begin{array}{l}\text { Bin Mean } \\
\text { Diameter } \\
(\mu \mathrm{m})\end{array}$ & $\begin{array}{l}\text { Diff. of the } \\
\log \text { of the } \\
\text { Size }\end{array}$ & Counts & $\begin{array}{l}\text { Weighted } \\
\text { Mean } \\
\text { Diameter }\end{array}$ \\
\hline $0.30-0.49$ & 0.38 & 0.21 & 42162 & \multirow{6}{*}{0.87} \\
\hline $0.50-0.99$ & 0.70 & 0.30 & 35823 & \\
\hline $1.00-1.99$ & 1.41 & 0.30 & 6215 & \\
\hline $2.00-4.99$ & 3.16 & 0.40 & 7089 & \\
\hline $5.00-9.99$ & 7.07 & 0.30 & 642 & \\
\hline$>10.00$ & 14.14 & 0.30 & 207 & \\
\hline \multicolumn{5}{|l|}{ Sample \#3 } \\
\hline \begin{tabular}{|l} 
Bin \\
Range \\
(um) \\
\end{tabular} & $\begin{array}{l}\text { Bin Mean } \\
\text { Diameter } \\
(\mu \mathrm{m})\end{array}$ & $\begin{array}{l}\text { Diff. of the } \\
\log \text { of the } \\
\text { Size }\end{array}$ & Counts & $\begin{array}{l}\text { Weighted } \\
\text { Mean } \\
\text { Diameter }\end{array}$ \\
\hline $0.30-0.49$ & 0.38 & 0.21 & 12523 & \\
\hline $0.50-0.99$ & 0.70 & 0.30 & 41383 & \\
\hline $1.00-1.99$ & 1.41 & 0.30 & 26039 & \\
\hline $2.00-4.99$ & 3.16 & 0.40 & 89253 & \\
\hline $5.00-9.99$ & 7.07 & 0.30 & 33015 & \\
\hline$>10.00$ & 14.14 & 0.30 & 38684 & \\
\hline
\end{tabular}

Sample \#5

\begin{tabular}{|c|c|c|c|c|}
\hline $\begin{array}{l}\text { Bin } \\
\text { Range } \\
\text { (um) }\end{array}$ & $\begin{array}{l}\text { Bin Mean } \\
\text { Diameter } \\
(\mu \mathrm{m})\end{array}$ & $\begin{array}{l}\text { Diff. of the } \\
\text { log of the } \\
\text { Size }\end{array}$ & Counts & $\begin{array}{l}\text { Weighted } \\
\text { Mean } \\
\text { Diameter }\end{array}$ \\
\hline $0.30-0.49$ & 0.38 & 0.21 & 27538 & \multirow{6}{*}{1.34} \\
\hline $0.50-0.99$ & 0.70 & 0.30 & 58636 & \\
\hline $1.00-1.99$ & 1.41 & 0.30 & 20482 & \\
\hline $2.00-4.99$ & 3.16 & 0.40 & 23941 & \\
\hline $5.00-9.99$ & 7.07 & 0.30 & 1566 & \\
\hline$>10.00$ & 14.14 & 0.30 & 787 & \\
\hline
\end{tabular}

Sample \#7

\begin{tabular}{|c|c|c|c|c|}
\hline $\begin{array}{l}\text { Bin } \\
\text { Range } \\
\text { (um) }\end{array}$ & $\begin{array}{l}\text { Bin Mean } \\
\text { Diameter } \\
(\omega \mathrm{m})\end{array}$ & $\begin{array}{l}\text { Diff. of the } \\
\text { log of the } \\
\text { Size }\end{array}$ & Counts & \begin{tabular}{|l} 
Weighted \\
Mean \\
Diameter
\end{tabular} \\
\hline $0.30-0.49$ & 0.38 & 0.21 & 36428 & \multirow{6}{*}{1.07} \\
\hline $0.50-0.99$ & 0.70 & 0.30 & 45704 & \\
\hline $1.00-1.99$ & $\overline{1.41}$ & 0.30 & 10867 & \\
\hline $2.00-4.99$ & 3.16 & 0.40 & 11843 & \\
\hline $5.00-9.99$ & 7.07 & 0.30 & 882 & \\
\hline$>10.00$ & 14.14 & 0.30 & 653 & \\
\hline
\end{tabular}

\begin{tabular}{||l|c|c|r|r||}
\hline $\begin{array}{l}\text { Bin } \\
\text { Range } \\
\text { (um) }\end{array}$ & $\begin{array}{l}\text { Bin Mean } \\
\text { Diameter } \\
(\mu \mathrm{m})\end{array}$ & $\begin{array}{l}\text { Diff. of the } \\
\text { log of the } \\
\text { Size }\end{array}$ & Counts & $\begin{array}{l}\text { Weighted } \\
\text { Mean } \\
\text { Diameter }\end{array}$ \\
\hline $0.30-0.49$ & 0.38 & 0.21 & 29796 & \\
\hline $0.50 \cdot 0.99$ & 0.70 & 0.30 & 52212 & \\
\hline $1.00-1.99$ & 1.41 & 0.30 & 16173 & \multirow{2}{*}{1.34} \\
\hline $2.00 \cdot 4.99$ & 3.16 & 0.40 & 18705 & \\
\hline $5.00-9.99$ & 7.07 & 0.30 & 1582 & \\
\hline$>10.00$ & 14.14 & 0.30 & 1354 & \\
\hline
\end{tabular}

Sample \#2

\begin{tabular}{|c|c|c|c|c|}
\hline $\begin{array}{l}\text { Bin } \\
\text { Range } \\
\text { (um) }\end{array}$ & $\begin{array}{l}\text { Bin Mean } \\
\text { Diameter } \\
(\mathrm{um})\end{array}$ & $\begin{array}{l}\text { Diff. of the } \\
\text { log of the } \\
\text { Size }\end{array}$ & Counts & $\begin{array}{l}\text { Weighted } \\
\text { Mean } \\
\text { Diameter }\end{array}$ \\
\hline $0.30-0.49$ & .38 & 0.21 & 22052 & \multirow{6}{*}{3.93} \\
\hline $0.50-0.99$ & .70 & 0.30 & 48292 & \\
\hline $1.00-1.99$ & 1.41 & 0.30 & 22315 & \\
\hline $2.00-4.99$ & 3.16 & 0.40 & 44177 & \\
\hline $5.00-9.99$ & 7.07 & 0.30 & 9779 & \\
\hline$>10.00$ & 14.14 & 0.30 & 28818 & \\
\hline
\end{tabular}

Sample \#4

\begin{tabular}{|c|c|c|c|c|}
\hline \begin{tabular}{|l} 
Bin \\
Rango \\
$(u \mathrm{~m})$
\end{tabular} & $\begin{array}{l}\text { Bin Mean } \\
\text { Diameter } \\
(\mu \mathrm{m})\end{array}$ & $\begin{array}{l}\text { Diff. of the } \\
\text { log of the } \\
\text { Size }\end{array}$ & Counts & $\begin{array}{l}\text { Weighted } \\
\text { Mean } \\
\text { Diameter }\end{array}$ \\
\hline $0.30-0.49$ & .38 & 0.21 & 17932 & \multirow{6}{*}{1.99} \\
\hline $0.50-0.99$ & .70 & 0.30 & 70863 & \\
\hline $1.00-1.99$ & 1.41 & 0.30 & 38109 & \\
\hline $2.00-4.99$ & 3.16 & 0.40 & 57855 & \\
\hline $5.00-9.99$ & 7.07 & 0.30 & 5736 & \\
\hline$>10.00$ & 14.14 & 0.30 & 3719 & \\
\hline
\end{tabular}

Sample \#6

\begin{tabular}{|c|c|c|c|c|}
\hline $\begin{array}{l}\text { Bin } \\
\text { Range } \\
\text { (um) }\end{array}$ & \begin{tabular}{|l|} 
Bin Mean \\
Diameter \\
$(\mathrm{em})$
\end{tabular} & $\begin{array}{l}\text { Diff. of the } \\
\log \text { of the } \\
\text { Size }\end{array}$ & Counts & $\begin{array}{l}\text { Weighted } \\
\text { Mean } \\
\text { Diameter }\end{array}$ \\
\hline $0.30-0.49$ & .38 & 0.21 & 23242 & \multirow{6}{*}{1.56} \\
\hline $0.50-0.99$ & .70 & 0.30 & 63124 & \\
\hline $1.00-1.99$ & 1.41 & 0.30 & 24936 & \\
\hline $2.00-4.99$ & 3.16 & 0.40 & 30218 & \\
\hline $5.00-9.99$ & 7.07 & 0.30 & 2446 & \\
\hline$>10.00$ & 14.14 & 0.30 & 1828 & \\
\hline
\end{tabular}

\begin{tabular}{|c|c|c|c|c|}
\hline \multicolumn{5}{|c|}{ Sample \#8 } \\
\hline $\begin{array}{l}\text { Bin } \\
\text { Range } \\
(\mathrm{sm})\end{array}$ & $\begin{array}{l}\text { Bin Mean } \\
\text { Diameter } \\
(\mathrm{um})\end{array}$ & $\begin{array}{l}\text { Diff. of the } \\
\text { log of the } \\
\text { Size }\end{array}$ & Counts & $\begin{array}{l}\text { Weighted } \\
\text { Mean } \\
\text { Diameter }\end{array}$ \\
\hline $0.30-0.49$ & .38 & 0.21 & $\overline{27761}$ & \multirow{6}{*}{1.40} \\
\hline $0.50-0.99$ & .70 & 0.30 & 55713 & \\
\hline $0.00-1.99$ & $\overline{1.41}$ & 0.30 & 18300 & \\
\hline $2.00-4.99$ & 3.16 & 0.40 & 21665 & \\
\hline $5.00-9.99$ & 7.07 & 0.30 & 1913 & \\
\hline$>10.00$ & 14.14 & 0.30 & 1427 & \\
\hline
\end{tabular}

Sample \#10

\begin{tabular}{|c|c|c|c|c|}
\hline $\begin{array}{l}\text { Bin } \\
\text { Range } \\
\text { (um) }\end{array}$ & \begin{tabular}{|l|} 
Bin Mean \\
Diameter \\
$(\mu \mathrm{m})$
\end{tabular} & $\begin{array}{l}\text { Diff. of the } \\
\log \text { of the } \\
\text { Size }\end{array}$ & Counts & $\begin{array}{l}\text { Weighted } \\
\text { Mean } \\
\text { Diameter }\end{array}$ \\
\hline $0.30-0.49$ & .38 & 0.21 & 24766 & \multirow{6}{*}{1.53} \\
\hline $0.50-0.99$ & .70 & 0.30 & 58742 & \\
\hline $1.00-1.99$ & 1.41 & 0.30 & 21073 & \\
\hline $2.00-4.99$ & 3.16 & 0.40 & 25848 & \\
\hline $5.00-9.99$ & 7.07 & 0.30 & 2459 & \\
\hline$>10.00$ & 14.14 & 0.30 & 1921 & \\
\hline
\end{tabular}




\begin{tabular}{|c|c|c|c|c|}
\hline $\begin{array}{l}\text { Bin } \\
\text { Range } \\
(\mu \mathrm{m})\end{array}$ & $\begin{array}{l}\text { Bin Mean } \\
\text { Diameter } \\
(\mu \mathrm{m})\end{array}$ & $\begin{array}{l}\text { Diff. of the } \\
\text { log of the } \\
\text { Size }\end{array}$ & Counts & $\begin{array}{l}\text { Weighted } \\
\text { Mean } \\
\text { Diameter }\end{array}$ \\
\hline $0.30 \cdot 0.49$ & 0.38 & 0.21 & 23556 & \multirow{6}{*}{1.51} \\
\hline $0.50-0.99$ & 0.70 & 0.30 & 61429 & \\
\hline $1.00-1.99$ & 1.41 & 0.30 & 23368 & \\
\hline $2.00-4.99$ & 3.16 & 0.40 & 29024 & \\
\hline 5.00 .9 .99 & 7.07 & 0.30 & 2356 & \\
\hline$>10.00$ & 14.14 & 0.30 & 1382 & \\
\hline
\end{tabular}

\begin{tabular}{|c|c|c|c|c|}
\hline \multicolumn{5}{|l|}{ Sample \#13 } \\
\hline \begin{tabular}{|l} 
Bin \\
Range \\
$(\mu \mathrm{m})$
\end{tabular} & $\begin{array}{l}\text { Bin Mean } \\
\text { Diameter } \\
(\mathrm{erm})\end{array}$ & $\begin{array}{l}\text { Diff. of the } \\
\text { log of the } \\
\text { Size }\end{array}$ & Counts & $\begin{array}{l}\text { Weighted } \\
\text { Mean } \\
\text { Diameter }\end{array}$ \\
\hline $0.30-0.49$ & 0.38 & 0.21 & 28626 & \multirow{6}{*}{1.30} \\
\hline $0.50-0.99$ & 0.70 & 0.30 & 55440 & \\
\hline $1.00-1.99$ & 1.41 & 0.30 & 18239 & \\
\hline $2.00-4.99$ & 3.16 & 0.40 & 21208 & \\
\hline $5.00 \cdot 9.99$ & 7.07 & 0.30 & 1581 & \\
\hline$>10.00$ & 14.14 & 0.30 & 705 & \\
\hline
\end{tabular}

Samole \#12

\begin{tabular}{||c|c|c|r|r|}
\hline $\begin{array}{l}\text { Bin } \\
\text { Range } \\
(\mathrm{m})\end{array}$ & $\begin{array}{l}\text { Bin Mean } \\
\text { Diameter } \\
(\mathrm{um})\end{array}$ & $\begin{array}{l}\text { Diff. of the } \\
\text { log of the } \\
\text { Size }\end{array}$ & Counts & $\begin{array}{l}\text { Weighted } \\
\text { Mean } \\
\text { Diameter }\end{array}$ \\
\hline $0.30-0.49$ & .38 & 0.21 & 23134 & \\
\hline $0.50-0.99$ & .70 & 0.30 & 63568 & \multirow{2}{*}{1.50} \\
\hline $1.00-1.99$ & 1.41 & 0.30 & 25599 & \\
\hline $2.00-4.99$ & 3.16 & 0.40 & 31628 & \\
\hline $5.00-9.99$ & 7.07 & 0.30 & 2402 & \\
\hline$>10.00$ & 14.14 & 0.30 & 1056 & \\
\hline
\end{tabular}

\begin{tabular}{|c|c|c|c|c|}
\hline \multicolumn{5}{|c|}{ Sample \#14 } \\
\hline $\begin{array}{l}\text { Bin } \\
\text { Range } \\
(\mu \mathrm{m})\end{array}$ & $\begin{array}{l}\text { Bin Mean } \\
\text { Diameter } \\
(\mathrm{em})\end{array}$ & $\begin{array}{l}\text { Diff. of the } \\
\text { log of the } \\
\text { Size }\end{array}$ & Counts & $\begin{array}{l}\text { Weighted } \\
\text { Mean } \\
\text { Diameter }\end{array}$ \\
\hline $0.30-0.49$ & .38 & 0.21 & 29128 & \multirow{6}{*}{1.28} \\
\hline $0.50-0.99$ & .70 & 0.30 & 54644 & \\
\hline $1.00-1.99$ & 1.41 & 0.30 & 18407 & \\
\hline $2.00-4.99$ & 3.16 & 0.40 & 20798 & \\
\hline $5.00-9.99$ & 7.07 & 0.30 & 1463 & \\
\hline$>10.00$ & 14.74 & 0.30 & 614 & \\
\hline
\end{tabular}




\section{Aerosol Particle Measurement Record}

Description of sample location 3706A Building, Resuspension Test, at $25 \mathrm{~cm}$, Date 8/12/94, Time sample started 10:49:54, Temperature 82.0 F, Humidity $38.2 \%$, Dew Point $53.9 \mathrm{~F}$, Average Wind Velocity (if applicable) $.00 \mathrm{~m} / \mathrm{s}$, \# of physical barriers between sample area and outside (if applicable) 1

\begin{tabular}{|c|c|c|c|c|}
\hline $\begin{array}{l}\text { Bin } \\
\text { Range } \\
\text { (um) }\end{array}$ & \begin{tabular}{|l|} 
Bin Mean \\
Diameter \\
$(\mu \mathrm{m})$
\end{tabular} & $\begin{array}{l}\text { Diff. of the } \\
\log \text { of the } \\
\text { Size }\end{array}$ & Counts & \begin{tabular}{|l} 
Weighted \\
Mean \\
Diameter
\end{tabular} \\
\hline $0.30-0.49$ & 0.38 & 0.21 & 58010 & \multirow{6}{*}{0.79} \\
\hline $0.50-0.99$ & 0.70 & 0.30 & 21610 & \\
\hline $1.00-1.99$ & 1.41 & 0.30 & 5642 & \\
\hline $2.00 \cdot 4.99$ & 3.16 & 0.40 & 6054 & \\
\hline $5.00-9.99$ & 7.07 & 0.30 & 649 & \\
\hline$>10.00$ & 14.14 & 0.30 & 252 & \\
\hline \multicolumn{5}{|l|}{ Sample \#3 } \\
\hline $\begin{array}{l}\text { Bin } \\
\text { Range } \\
(\mu \mathrm{m})\end{array}$ & \begin{tabular}{|l|} 
Bin Mean \\
Diameter \\
$(u m)$
\end{tabular} & $\begin{array}{l}\text { Diff. of the } \\
\text { log of the } \\
\text { Size }\end{array}$ & Counts & $\begin{array}{l}\text { Weighted } \\
\text { Mean } \\
\text { Diameter }\end{array}$ \\
\hline $0.30-0.49$ & 0.38 & 0.21 & 2832 & \multirow{6}{*}{8.16} \\
\hline $0.50-0.99$ & 0.70 & 0.30 & 8246 & \\
\hline $1.00-1.99$ & 1.41 & 0.30 & 11206 & \\
\hline $2.00-4.99$ & 3.16 & 0.40 & 84682 & \\
\hline $5.00-9.99$ & 7.07 & 0.30 & 65540 & \\
\hline$>10.00$ & 14.14 & 0.30 & 109591 & \\
\hline \multicolumn{5}{|l|}{ Sample \#5 } \\
\hline $\begin{array}{l}\text { Bin } \\
\text { Range } \\
(\mu \mathrm{m})\end{array}$ & \begin{tabular}{|l} 
Bin Mean \\
Diameter \\
$(u m)$
\end{tabular} & $\begin{array}{l}\text { Diff. of the } \\
\log \text { of the } \\
\text { Size }\end{array}$ & Counts & \begin{tabular}{|l|} 
Weighted \\
Mean \\
Diameter
\end{tabular} \\
\hline $0.30 \cdot 0.49$ & 0.38 & 0.21 & 20422 & \multirow{6}{*}{2.52} \\
\hline $0.50-0.99$ & 0.70 & 0.30 & 37288 & \\
\hline $1.00-1.99$ & 1.41 & 0.30 & 31042 & \\
\hline $2.00-4.99$ & 3.16 & 0.40 & 67424 & \\
\hline $5.00-9.99$ & 7.07 & 0.30 & 11116 & \\
\hline$>10.00$ & 14.14 & 0.30 & 4562 & \\
\hline \multicolumn{5}{|l|}{ Sample \#7 } \\
\hline \begin{tabular}{|l|} 
Bin \\
Range \\
$(\mu \mathrm{m})$ \\
\end{tabular} & \begin{tabular}{|l|} 
Bin Mean \\
Diameter \\
(um)
\end{tabular} & $\begin{array}{l}\text { Diff. of the } \\
\text { log of the } \\
\text { Size }\end{array}$ & Counts & \begin{tabular}{|l|} 
Weighted \\
Mean \\
Diameter
\end{tabular} \\
\hline $0.30-0.49$ & 0.38 & 0.21 & 40824 & \multirow{6}{*}{1.41} \\
\hline $0.50-0.99$ & 0.70 & 0.30 & 28324 & \\
\hline $1.00-1.99$ & 1.41 & 0.30 & 12909 & \\
\hline $2.00-4.99$ & 3.16 & 0.40 & 18241 & \\
\hline $5.00-9.99$ & 7.07 & 0.30 & 2554 & \\
\hline$>10.00$ & 14.14 & 0.30 & 1256 & \\
\hline \multicolumn{5}{|l|}{ Sample \#9 } \\
\hline \begin{tabular}{|l|} 
Bin \\
Rangs \\
(um) \\
\end{tabular} & \begin{tabular}{|l} 
Bin Mean \\
Diameter \\
$(\mu \mathrm{m})$
\end{tabular} & $\begin{array}{l}\text { Diff. of the } \\
\text { log of the } \\
\text { Size }\end{array}$ & Counts & \begin{tabular}{|l|} 
Weighted \\
Mean \\
Diameter
\end{tabular} \\
\hline $0.30-0.49$ & 0.38 & 0.21 & 42097 & \multirow{6}{*}{1.37} \\
\hline $0.50-0.99$ & 0.70 & 0.30 & 28421 & \\
\hline $1.00-1.99$ & 1.41 & 0.30 & 12456 & \\
\hline $2.00-4.99$ & 3.16 & 0.40 & 17354 & \\
\hline $5.00-9.99$ & 7.07 & 0.30 & 2494 & \\
\hline$>10.00$ & 14.14 & 0.30 & 1194 & \\
\hline
\end{tabular}

Sample \#2

\begin{tabular}{|c|c|c|c|c|}
\hline $\begin{array}{l}\text { Bin } \\
\text { Range } \\
\text { (um) }\end{array}$ & \begin{tabular}{|l|} 
Bin Mean \\
Diameter \\
(um)
\end{tabular} & $\begin{array}{l}\text { Diff. of the } \\
\log \text { of the } \\
\text { Size }\end{array}$ & Counts & $\begin{array}{l}\text { Weighted } \\
\text { Mean } \\
\text { Diameter }\end{array}$ \\
\hline $0.30-0.49$ & .38 & 0.21 & 7281 & \\
\hline $0.50-0.99$ & .70 & 0.30 & 9466 & \\
\hline $1.00-1.99$ & 1.41 & 0.30 & 8081 & \\
\hline $2.00-4.99$ & 3.16 & 0.40 & 41263 & \\
\hline $5.00-9.99$ & 7.07 & 0.30 & 29647 & \\
\hline$>10.00$ & 14.14 & 0.30 & 147890 & \\
\hline
\end{tabular}

\begin{tabular}{|c|c|c|c|c|}
\hline \multicolumn{5}{|c|}{ Sample \#4 } \\
\hline $\begin{array}{l}\text { Bin } \\
\text { Range } \\
(\mathrm{em})\end{array}$ & \begin{tabular}{|l|} 
Bin Mean \\
Diameter \\
$(u \mathrm{~m})$
\end{tabular} & $\begin{array}{l}\text { Diff. of the } \\
\text { log of the } \\
\text { Size }\end{array}$ & Counts & $\begin{array}{l}\text { Weighted } \\
\text { Mean } \\
\text { Diameter }\end{array}$ \\
\hline $0.30-0.49$ & .38 & 0.21 & 10533 & \multirow{6}{*}{3.88} \\
\hline $0.50-0.99$ & .70 & 0.30 & 28479 & \\
\hline $1.00-1.99$ & 1.41 & 0.30 & 33462 & \\
\hline $2.00-4.99$ & 3.16 & 0.40 & 128117 & \\
\hline $5.00-9.99$ & 7.07 & 0.30 & 37113 & \\
\hline$>10.00$ & 14.14 & 0.30 & 17951 & \\
\hline
\end{tabular}

\begin{tabular}{|c|c|c|c|c|}
\hline \multicolumn{5}{|l|}{ Sample $\$ 6$} \\
\hline $\begin{array}{l}\text { Bin } \\
\text { Range } \\
(u m)\end{array}$ & \begin{tabular}{|l} 
Bin Mean \\
Diameter \\
$(\mu \mathrm{m})$
\end{tabular} & $\begin{array}{l}\text { Diff. of the } \\
\log \text { of the } \\
\text { Size }\end{array}$ & Counts. & $\begin{array}{l}\text { Weighted } \\
\text { Mean } \\
\text { Diameter }\end{array}$ \\
\hline $0.30-0.49$ & .38 & 0.21 & 30391 & \multirow{6}{*}{1.86} \\
\hline $0.50-0.99$ & .70 & 0.30 & 32965 & \\
\hline $1.00-1.99$ & 1.41 & 0.30 & 19671 & \\
\hline $2.00-4.99$ & 3.16 & 0.40 & $\overline{32112}$ & \\
\hline $5.00-9.99$ & 7.07 & 0.30 & 4538 & \\
\hline$>10.00$ & 14.14 & 0.30 & 2165 & \\
\hline
\end{tabular}

\begin{tabular}{|c|c|c|c|c|}
\hline \multicolumn{5}{|l|}{ Sample \#8 } \\
\hline $\begin{array}{l}\text { Bin } \\
\text { Range } \\
(\mu \mathrm{m})\end{array}$ & $\begin{array}{l}\text { Bin Mean } \\
\text { Diameter } \\
(\mu \mathrm{m})\end{array}$ & $\begin{array}{l}\text { Diff. of the } \\
\text { log of the } \\
\text { Size }\end{array}$ & Counts & $\begin{array}{l}\text { Woighted } \\
\text { Mean } \\
\text { Diameter }\end{array}$ \\
\hline $0.30-0.49$ & .38 & 0.21 & 42427 & \multirow{6}{*}{1.35} \\
\hline $0.50-0.99$ & .70 & 0.30 & 28228 & \\
\hline $0.00-1.99$ & 1.41 & 0.30 & 12571 & \\
\hline $2.00-4.99$ & 3.16 & 0.40 & 17283 & \\
\hline $5.00-9.99$ & 7.07 & 0.30 & 2287 & \\
\hline$>10.00$ & 14.14 & 0.30 & 1107 & \\
\hline
\end{tabular}

\begin{tabular}{|c|c|c|c|c|}
\hline \multicolumn{5}{|c|}{ Sample \#10 } \\
\hline \begin{tabular}{|l} 
Bin \\
Range \\
$(\mu \mathrm{m})$
\end{tabular} & \begin{tabular}{|l} 
Bin Mean \\
Diameter \\
$(\mu \mathrm{m})$
\end{tabular} & $\begin{array}{l}\text { Diff. of the } \\
\text { log of the } \\
\text { Size }\end{array}$ & Counts & $\begin{array}{l}\text { Weightec } \\
\text { Mean } \\
\text { Diameter }\end{array}$ \\
\hline $0.30-0.49$ & .38 & 0.21 & 40988 & \multirow{6}{*}{1.40} \\
\hline $0.50-0.99$ & .70 & 0.30 & 28871 & \\
\hline $1.00-1.99$ & 9.41 & 0.30 & $\overline{13314}$ & \\
\hline $2.00-4.99$ & 3.16 & 0.40 & 19216 & \\
\hline $5.00-9.99$ & 7.07 & 0.30 & 2492 & \\
\hline$>10.00$ & 14.14 & 0.30 & 1071 & \\
\hline
\end{tabular}


Sample \#11

\begin{tabular}{|c|c|c|c|c|}
\hline $\begin{array}{l}\text { Bin } \\
\text { Range } \\
\text { (um) }\end{array}$ & \begin{tabular}{|l} 
Bin Mean \\
Diameter \\
$(\mathrm{um})$
\end{tabular} & $\begin{array}{l}\text { Diff. of the } \\
\log \text { of the } \\
\text { Size }\end{array}$ & Counts & $\begin{array}{l}\text { Weighted } \\
\text { Mean } \\
\text { Diameter }\end{array}$ \\
\hline $0.30-0.49$ & 0.38 & 0.21 & 33525 & \multirow{6}{*}{1.68} \\
\hline $0.50-0.99$ & 0.70 & 0.30 & 32250 & \\
\hline $1.00-1.99$ & 1.41 & 0.30 & 18055 & \\
\hline $2.00-4.99$ & 3.16 & 0.40 & 28454 & \\
\hline $5.00-9.99$ & 7.07 & 0.30 & 3892 & \\
\hline$>10.00$ & 14.14 & 0.30 & 1363 & \\
\hline
\end{tabular}

\begin{tabular}{|c|c|c|c|c|}
\hline \multicolumn{5}{|l|}{ Sample \#13 } \\
\hline $\begin{array}{l}\text { Bin } \\
\text { Range } \\
(\text { (um) }\end{array}$ & $\begin{array}{l}\text { Bin Mean } \\
\text { Diameter } \\
(\mathrm{fm})\end{array}$ & $\begin{array}{l}\text { Diff. of the } \\
\log \text { of the } \\
\text { Size }\end{array}$ & Counts & $\begin{array}{l}\text { Weighted } \\
\text { Mean } \\
\text { Diameter }\end{array}$ \\
\hline $0.30-0.49$ & 0.38 & 0.21 & 34212 & \multirow{6}{*}{1.64} \\
\hline $0.50-0.99$ & 0.70 & 0.30 & 32098 & \\
\hline $1.00-1.99$ & 1.41 & 0.30 & 17712 & \\
\hline $2,00-4.99$ & 3.16 & 0.40 & 28333 & \\
\hline $5.00-9.99$ & 7.07 & 0.30 & 3652 & \\
\hline$>10.00$ & 14.14 & 0.30 & 1133 & \\
\hline
\end{tabular}

Sample \#12

\begin{tabular}{|c|c|c|c|c|}
\hline $\begin{array}{l}\text { Bin } \\
\text { Range } \\
(\mu \mathrm{m})\end{array}$ & $\begin{array}{l}\text { Bin Mean } \\
\text { Diameter } \\
(\omega \mathrm{m}) \\
\end{array}$ & \begin{tabular}{|l} 
Diff. of the \\
log of the \\
Size
\end{tabular} & Counts & $\begin{array}{l}\text { Weighted } \\
\text { Mean } \\
\text { Diameter }\end{array}$ \\
\hline $0.30-0.49$ & .38 & 0.21 & 32870 & \multirow{6}{*}{1.69} \\
\hline $0.50-0.99$ & .70 & 0.30 & 32338 & \\
\hline $1.00-1.99$ & 1.41 & 0.30 & 18689 & \\
\hline $2.00-4.99$ & 3.16 & 0.40 & 29515 & \\
\hline $5.00-9.99$ & 7.07 & 0.30 & 3899 & \\
\hline$>10.00$ & 14.14 & 0.30 & 1303 & \\
\hline
\end{tabular}

\begin{tabular}{|c|c|c|c|c|}
\hline \multicolumn{5}{|c|}{ Sample $\# 14$} \\
\hline $\begin{array}{l}\text { Bin } \\
\text { Range } \\
\text { (um) }\end{array}$ & $\begin{array}{l}\text { Bin Mean } \\
\text { Diameter } \\
(\mathrm{um})\end{array}$ & $\begin{array}{l}\text { Diff. of the } \\
\log \text { of the } \\
\text { Size }\end{array}$ & Counts & $\begin{array}{l}\text { Weighted } \\
\text { Mean } \\
\text { Diameter }\end{array}$ \\
\hline $0.30-0.49$ & .38 & 0.21 & 34556 & \multirow{6}{*}{1.64} \\
\hline $0.50-0.99$ & .70 & 0.30 & 32354 & \\
\hline $1.00-1.99$ & 1.41 & 0.30 & 18339 & \\
\hline $2.00-4.99$ & 3.16 & 0.40 & 29368 & \\
\hline $5.00-9.99$ & 7.07 & 0.30 & 3855 & \\
\hline$>10.00$ & 14.74 & 0.30 & 1042 & \\
\hline
\end{tabular}




\section{Aerosol Particle Measurement Record}

Description of sample location 3706A Building, Resuspension Test, at $0.5 \mathrm{~m}$, Date 8/12/94, Time sample started 12:36:11, Temperature 83.1 F, Humidity $36.8 \%$, Dew Point 53.9 F, Average Wind Velocity (if applicable) $.00 \mathrm{~m} / \mathrm{s}$, \# of physical barriers between sample area and outside (if applicable) 1

Sample \#1

\begin{tabular}{|c|c|c|c|c|}
\hline \begin{tabular}{|l} 
Bin \\
Range \\
$(\mu \mathrm{m})$
\end{tabular} & \begin{tabular}{|l} 
Bin Mean \\
Diameter \\
$(u m)$
\end{tabular} & $\begin{array}{l}\text { Diff. of the } \\
\log \text { of the } \\
\text { Size }\end{array}$ & Counts & $\begin{array}{l}\text { Weighted } \\
\text { Mean } \\
\text { Diameter }\end{array}$ \\
\hline $0.30-0.49$ & 0.38 & 0.21 & 58971 & \multirow{6}{*}{0.62} \\
\hline $0.50-0.99$ & 0.70 & 0.30 & 40639 & \\
\hline $1.00-1.99$ & 1.41 & 0.30 & 3254 & \\
\hline $2.00-4.99$ & 3.16 & 0.40 & 2463 & \\
\hline $5.00-9.99$ & 7.07 & 0.30 & 127 & \\
\hline$>10.00$ & 14.14 & 0.30 & 67 & \\
\hline \multicolumn{5}{|l|}{ Sample \#3 } \\
\hline \begin{tabular}{|l} 
Bin \\
Range \\
(um)
\end{tabular} & \begin{tabular}{|l} 
Bin Mean \\
Diameter \\
$(\mu \mathrm{m})$
\end{tabular} & $\begin{array}{l}\text { Diff. of the } \\
\text { log of the } \\
\text { Size }\end{array}$ & Counts & \begin{tabular}{|l} 
Weighted \\
Mean \\
Diameter
\end{tabular} \\
\hline $0.30-0.49$ & 0.38 & 0.21 & 4296 & \\
\hline $0.50-0.99$ & 0.70 & 0.30 & 28606 & \\
\hline $1.00-1.99$ & 1.41 & 0.30 & 29318 & \\
\hline $2.00-4.99$ & 3.16 & 0.40 & 136496 & \\
\hline $5.00-9.99$ & 7.07 & 0.30 & 53895 & \\
\hline$>10.00$ & 14.14 & 0.30 & 61771 & \\
\hline \multicolumn{5}{|l|}{ Sample \#5 } \\
\hline \begin{tabular}{|l} 
Bin \\
Range \\
( $\mu \mathrm{m})$ \\
\end{tabular} & \begin{tabular}{|l}
$\begin{array}{l}\text { Bin Mean } \\
\text { Diameter } \\
(\text { (rm) }\end{array}$ \\
\end{tabular} & \begin{tabular}{|l} 
Diff. of thie \\
$\log$ of the \\
Size
\end{tabular} & Counts & $\begin{array}{l}\text { Weighted } \\
\text { Mean } \\
\text { Diameter }\end{array}$ \\
\hline $0.30 \cdot 0.49$ & 0.38 & \begin{tabular}{|l|}
0.21 \\
\end{tabular} & 29661 & \multirow{6}{*}{1.67} \\
\hline $0.50-0.99$ & 0.70 & 0.30 & 59322 & \\
\hline $1.00-1.99$ & 1.41 & 0.30 & 23472 & \\
\hline $2.00-4.99$ & 3.16 & 0.40 & 35201 & \\
\hline $5.00-9.99$ & 7.07 & 0.30 & 3560 & \\
\hline$>10.00$ & 14.14 & 0.30 & 2436 & \\
\hline \multicolumn{5}{|l|}{ Sample \#7 } \\
\hline \begin{tabular}{|l} 
Bin \\
Range \\
$(\mu \mathrm{m})$ \\
\end{tabular} & \begin{tabular}{|l|} 
Bin Mean \\
Diameter \\
$(u / m)$
\end{tabular} & \begin{tabular}{|l|} 
Diff. of the \\
log of the \\
Size
\end{tabular} & Counts & \begin{tabular}{|l|} 
Weighted \\
Mean \\
Diameter
\end{tabular} \\
\hline \begin{tabular}{|c|c|}
$0.30 \cdot 0.49$ \\
\end{tabular} & 0.38 & 0.21 & 20719 & \multirow{6}{*}{1.77} \\
\hline $0.50-0.99$ & 0.70 & 0.30 & 72345 & \\
\hline $1.00-1.99$ & 1.41 & 0.30 & 35187 & \\
\hline $2.00-4.99$ & 3.16 & 0.40 & 50053 & \\
\hline $5.00-9.99$ & 7.07 & 0.30 & 4244 & \\
\hline$>10.00$ & 14.14 & 0.30 & 2230 & \\
\hline \multicolumn{5}{|l|}{ Sample \#9 } \\
\hline $\begin{array}{l}\text { Bin } \\
\text { Range } \\
(\mathrm{sm})\end{array}$ & \begin{tabular}{|l|}
$\begin{array}{l}\text { Bin Mean } \\
\text { Diameter } \\
(\mu m)\end{array}$ \\
\end{tabular} & $\begin{array}{l}\text { Diff. of the } \\
\log \text { of the } \\
\text { Size }\end{array}$ & Counts & $\begin{array}{l}\text { Weighted } \\
\text { Mean } \\
\text { Diameter }\end{array}$ \\
\hline $0.30-0.49$ & $\begin{array}{l}0.38 \\
\end{array}$ & 0.21 & 27357 & \multirow{6}{*}{1.42} \\
\hline $0.50-0.99$ & 0.70 & 0.30 & 61505 & \\
\hline $1.00-1.99$ & 1.41 & 0.30 & 21702 & \\
\hline $2.00-4.99$ & 3.16 & 0.40 & 26267 & \\
\hline $5.00-9.99$ & 7.07 & 0.30 & 2146 & \\
\hline$>10.00$ & 14.14 & 0.30 & 1173 & \\
\hline
\end{tabular}

Sample \#2

\begin{tabular}{|c|c|c|c|c|}
\hline $\begin{array}{l}\text { Bin } \\
\text { Rangs } \\
(u m)\end{array}$ & $\begin{array}{l}\text { Bin Mean } \\
\text { Diameter } \\
(\mu \mathrm{m})\end{array}$ & $\begin{array}{l}\text { Diff. of the } \\
\text { log of the } \\
\text { Size }\end{array}$ & Counts & $\begin{array}{l}\text { Weighted } \\
\text { Mean } \\
\text { Diameter }\end{array}$ \\
\hline $0.30-0.49$ & .38 & 0.21 & 21372 & \multirow{6}{*}{4.38} \\
\hline $0.50-0.99$ & .70 & 0.30 & 43594 & \\
\hline $1.00-1.99$ & 1.41 & 0.30 & 21323 & \\
\hline $2.00-4.99$ & 3.16 & 0.40 & 51724 & \\
\hline $5.00-9.99$ & 7.07 & 0.30 & 16427 & \\
\hline$>10.00$ & 14.14 & 0.30 & 33579 & \\
\hline
\end{tabular}
Sample \#द

\begin{tabular}{|c|c|c|c|c|}
\hline \begin{tabular}{|l|} 
Bin \\
Range \\
$(\mu \mathrm{m})$
\end{tabular} & \begin{tabular}{|l} 
Bin Mean \\
Diameter \\
$(u r m)$
\end{tabular} & $\begin{array}{l}\text { Diff. of the } \\
\text { log of the } \\
\text { Size }\end{array}$ & Counts & $\begin{array}{l}\text { Weighted } \\
\text { Mean } \\
\text { Diameter }\end{array}$ \\
\hline $0.30-0.49$ & .38 & 0.21 & 5861 & \multirow{6}{*}{.4 .17} \\
\hline $0.50-0.99$ & .70 & 0.30 & 41748 & \\
\hline $1.00-1.99$ & 1.41 & 0.30 & 42307 & \\
\hline $2.00-4.99$ & 3.16 & 0.40 & 156013 & \\
\hline $5.00-9.99$ & 7.07 & 0.30 & 40964 & \\
\hline$>10.00$ & 14.14 & 0.30 & 32451 & \\
\hline
\end{tabular}
Sample \#6

\begin{tabular}{|c|c|c|c|c|}
\hline \begin{tabular}{|l} 
Bin \\
Range \\
$(\mu \mathrm{m})$
\end{tabular} & $\begin{array}{l}\text { Bin Mean } \\
\text { Diameter } \\
(u m)\end{array}$ & $\begin{array}{l}\text { Diff. of the } \\
\text { log of the } \\
\text { Size }\end{array}$ & Counts & $\begin{array}{l}\text { Weighted } \\
\text { Mean } \\
\text { Diameter }\end{array}$ \\
\hline $0.30-0.49$ & .38 & 0.21 & 29686 & \multirow{6}{*}{1.52} \\
\hline $0.50-0.99$ & .70 & 0.30 & 62094 & \\
\hline $1.00-1.99$ & 1.41 & 0.30 & 23669 & \\
\hline $2.00-4.99$ & 3.16 & 0.40 & 30279 & \\
\hline $5.00-9.99$ & 7.07 & 0.30 & 2608 & \\
\hline$>10.00$ & 14.14 & 0.30 & 1798 & \\
\hline
\end{tabular}

\begin{tabular}{|c|c|c|c|c|}
\hline \multicolumn{5}{|l|}{ Sample \#8 } \\
\hline $\begin{array}{l}\text { Bin } \\
\text { Range } \\
(\mu \mathrm{m})\end{array}$ & $\begin{array}{l}\text { Bin Mean } \\
\text { Diameter } \\
(\mu \mathrm{m})\end{array}$ & $\begin{array}{l}\text { Diff. of the } \\
\text { log of the } \\
\text { Size }\end{array}$ & Counts & $\begin{array}{l}\text { Weighted } \\
\text { Mean } \\
\text { Diameter }\end{array}$ \\
\hline $0.30-0.49$ & .38 & 0.21 & 22594 & \multirow{6}{*}{1.64} \\
\hline $0.50-0.99$ & .70 & 0.30 & 69461 & \\
\hline $0.00-1.99$ & 1.41 & 0.30 & 29913 & \\
\hline $2.00-4.99$ & 3.16 & 0.40 & 39188 & \\
\hline $5.00-9.99$ & 7.07 & 0.30 & 3220 & \\
\hline$>10.00$ & 14.14 & 0.30 & 1856 & \\
\hline
\end{tabular}

\begin{tabular}{|c|c|c|c|c|}
\hline \multicolumn{5}{|c|}{ Sample \#10 } \\
\hline \begin{tabular}{|l} 
Bin \\
Range \\
$(\mu \mathrm{m})$
\end{tabular} & $\begin{array}{l}\text { Bin Mean } \\
\text { Diameter } \\
\text { (um) }\end{array}$ & $\begin{array}{l}\text { Diff. of the } \\
\text { log of the } \\
\text { Size }\end{array}$ & Counts & $\begin{array}{l}\text { Weighted } \\
\text { Mean } \\
\text { Diameter }\end{array}$ \\
\hline $0.30-0.49$ & .38 & 0.21 & 22833 & \multirow{6}{*}{1.58} \\
\hline $0.50-0.99$ &.$\overline{70}$ & 0.30 & 69540 & \\
\hline $1.00-1.99$ & 1.41 & 0.30 & 29079 & \\
\hline $2.00-4.99$ & 3.16 & 0.40 & 36627 & \\
\hline $5.00-9.99$ & 7.07 & 0.30 & 2902 & \\
\hline$>10.00$ & 14.14 & 0.30 & 1592 & \\
\hline
\end{tabular}


Sample \#11

\begin{tabular}{|c|c|c|c|c|}
\hline $\begin{array}{l}\text { Bin } \\
\text { Range } \\
\text { (um) }\end{array}$ & \begin{tabular}{|l|} 
Bin Mean \\
Diameter \\
$($ (rm)
\end{tabular} & $\begin{array}{l}\text { Diff. of the } \\
\log \text { of the } \\
\text { Size }\end{array}$ & Counts & $\begin{array}{l}\text { Weighted } \\
\text { Mean } \\
\text { Diameter }\end{array}$ \\
\hline $0.30-0.49$ & 0.38 & 0.21 & 23920 & \multirow{6}{*}{1.55} \\
\hline $0.50-0.99$ & 0.70 & 0.30 & 71378 & \\
\hline $1.00-1.99$ & $\overline{1.41}$ & 0.30 & 29913 & \\
\hline $2.00-4.99$ & 3.16 & 0.40 & 37409 & \\
\hline $5.00-9.99$ & 7.07 & 0.30 & 2698 & \\
\hline$>10.00$ & 14.14 & 0.30 & 1399 & \\
\hline
\end{tabular}

Sample \#13

\begin{tabular}{|c|c|c|c|c|}
\hline $\begin{array}{l}\text { Bin } \\
\text { Range } \\
\text { (um) }\end{array}$ & \begin{tabular}{|l|} 
Bin Mean \\
Diameter \\
$(u m)$
\end{tabular} & $\begin{array}{l}\text { Diff. of the } \\
\log \text { of the } \\
\text { Size }\end{array}$ & Counts & \begin{tabular}{|l} 
Weighted \\
Mean \\
Diameter
\end{tabular} \\
\hline $0.30-0.49$ & 0.38 & 0.21 & 25464 & \multirow{6}{*}{1.45} \\
\hline $0.50-0.99$ & 0.70 & 0.30 & 66935 & \\
\hline $1.00-1.99$ & 1.41 & 0.30 & 26063 & \\
\hline $2.00-4.99$ & 3.16 & 0.40 & 31215 & \\
\hline $5.00-9.99$ & 7.07 & 0.30 & 2178 & \\
\hline$>10.00$ & 14.14 & 0.30 & 988 & \\
\hline
\end{tabular}

Samole \#12

\begin{tabular}{|c|c|c|c|c|}
\hline \begin{tabular}{|l|} 
Bin \\
Range \\
$(u m)$
\end{tabular} & \begin{tabular}{|l|} 
Bin Mean \\
Diameter \\
$($ (um)
\end{tabular} & $\begin{array}{l}\text { Diff. of the } \\
\text { log of the } \\
\text { Size }\end{array}$ & Counts & \begin{tabular}{|l|} 
Weighted \\
Mean \\
Diameter
\end{tabular} \\
\hline $0.30-0.49$ & .38 & 0.21 & 22676 & \multirow{6}{*}{1.56} \\
\hline $0.50-0.99$ & .70 & 0.30 & 69311 & \\
\hline $1.00-1.99$ & 1.41 & 0.30 & 29172 & \\
\hline $2.00-4.99$ & 3.16 & 0.40 & 36384 & \\
\hline $5.00-9.99$ & 7.07 & 0.30 & 2783 & \\
\hline$>10.00$ & 14.14 & 0.30 & 1378 & \\
\hline
\end{tabular}

\begin{tabular}{|c|c|c|c|c|}
\hline \multicolumn{5}{|c|}{ Sample \#14 } \\
\hline $\begin{array}{l}\text { Bin } \\
\text { Range } \\
(\mu \mathrm{m})\end{array}$ & $\begin{array}{l}\text { Bin Mean } \\
\text { Diameter } \\
\text { (um) }\end{array}$ & $\begin{array}{l}\text { Diff. of the } \\
\text { log of the } \\
\text { Size }\end{array}$ & Counts & $\begin{array}{l}\text { Weighted } \\
\text { Mean } \\
\text { Diameter }\end{array}$ \\
\hline $0.30-0.49$ & .38 & 0.21 & 22498 & \multirow{6}{*}{1.54} \\
\hline $0.50-0.99$ & .70 & 0.30 & 70003 & \\
\hline $1.00-1.99$ & 1.41 & 0.30 & 30140 & \\
\hline $2.00-4.99$ & 3.16 & 0.40 & 37462 & \\
\hline $5.00-9.99$ & 7.07 & 0.30 & 2622 & \\
\hline$>10.00$ & 14.14 & 0.30 & 1065 & \\
\hline
\end{tabular}




\section{Aerosol Particle Measurement Record}

Description of sample location 3706 A Building, Resuspension Test, at $25 \mathrm{~cm}$, Date 8/12/94, Time sample started 12:36:11, Temperature 83.1 F, Humidity $36.8 \%$, Dew Point 53.9 F, Average Wind Velocity (if applicable) $.00 \mathrm{~m} / \mathrm{s}$, \# of physical barriers between sample area and outside (if applicable) 1

Sample \#1

\begin{tabular}{|c|c|c|c|c|}
\hline $\begin{array}{l}\text { Bin } \\
\text { Range } \\
(\mu \mathrm{m})\end{array}$ & \begin{tabular}{|l|} 
Bin Mean \\
Diameter \\
$(\mu \mathrm{m})$
\end{tabular} & $\begin{array}{l}\text { Diff. of the } \\
\log \text { of the } \\
\text { Size }\end{array}$ & Counts & $\begin{array}{l}\text { Weighted } \\
\text { Mean } \\
\text { Diameter }\end{array}$ \\
\hline $0.30-0.49$ & 0.38 & 0.21 & 69987 & \multirow{6}{*}{0.65} \\
\hline $0.50-0.99$ & 0.70 & 0.30 & 24241 & \\
\hline $1.00-1.99$ & 1.41 & 0.30 & 4886 & \\
\hline $2.00-4.99$ & 3.16 & 0.40 & 3678 & \\
\hline $5.00-9.99$ & 7.07 & 0.30 & 330 & \\
\hline$>70.00$ & 14.14 & 0.30 & 158 & \\
\hline \multicolumn{5}{|l|}{ Sample \#3 } \\
\hline \begin{tabular}{|l} 
Bin \\
Range \\
$(\mu \mathrm{m})$
\end{tabular} & \begin{tabular}{|l} 
Bin Mean \\
Diameter \\
( $\mu \mathrm{m})$
\end{tabular} & $\begin{array}{l}\text { Diff. of the } \\
\log \text { of the } \\
\text { size }\end{array}$ & Counts & $\begin{array}{l}\text { Weighted } \\
\text { Mean } \\
\text { Diameter }\end{array}$ \\
\hline $0.30-0.49$ & 0.38 & 0.21 & 3480 & \multirow{6}{*}{7.37} \\
\hline $0.50-0.99$ & 0.70 & 0.30 & 10017 & \\
\hline $1.00-1.99$ & 1.41 & 0.30 & 13874 & \\
\hline $2.00-4.99$ & 3.16 & 0.40 & 92423 & \\
\hline $5.00-9.99$ & 7.07 & 0.30 & 60036 & \\
\hline$>10.00$ & 14.14 & 0.30 & 85808 & \\
\hline \multicolumn{5}{|l|}{ Sample \#5 } \\
\hline $\begin{array}{l}\text { Bin } \\
\text { Range } \\
(\mu \mathrm{m}) \\
\end{array}$ & \begin{tabular}{|l}
$\begin{array}{l}\text { Bin Mean } \\
\text { Diameter } \\
(\mu \mathrm{m})\end{array}$ \\
\end{tabular} & $\begin{array}{l}\text { Diff. of the } \\
\text { log of the } \\
\text { Size }\end{array}$ & Counts & $\begin{array}{l}\text { Weighted } \\
\text { Mean } \\
\text { Diameter }\end{array}$ \\
\hline $0.30-0.49$ & 0.38 & 0.21 & 26712 & \multirow{6}{*}{2.34} \\
\hline $0.50-0.99$ & 0.70 & 0.30 & 36492 & \\
\hline $1.00-1.99$ & 1.41 & 0.30 & 27144 & \\
\hline $2.00 \cdot 4.99$ & 3.16 & 0.40 & 54970 & \\
\hline $5.00-9.99$ & 7.07 & 0.30 & 9026 & \\
\hline$>10.00$ & 14.14 & 0.30 & 4151 & \\
\hline \multicolumn{5}{|l|}{ Sample \#7 } \\
\hline $\begin{array}{l}\text { Bin } \\
\text { Range } \\
(\mu \mathrm{m})\end{array}$ & 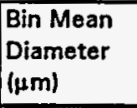 & $\begin{array}{l}\text { Diff. of the } \\
\text { log of the } \\
\text { Size }\end{array}$ & Counts & $\begin{array}{l}\text { Weighted } \\
\text { Mean } \\
\text { Diameter }\end{array}$ \\
\hline $0.30-0.49$ & 0.38 & 0.21 & 31897 & \multirow{6}{*}{2.05} \\
\hline $0.50-0.99$ & 0.70 & 0.30 & 34685 & \\
\hline $1.00-1.99$ & 1.41 & 0.30 & 23129 & \\
\hline $2.00 \cdot 4.99$ & 3.16 & 0.40 & 44570 & \\
\hline $5.00-9.99$ & 7.07 & 0.30 & 6832 & \\
\hline$>10.00$ & 14.14 & 0.30 & 2583 & \\
\hline
\end{tabular}

Sample \#2

\begin{tabular}{|c|c|c|c|c|}
\hline \begin{tabular}{|l} 
Bin \\
Range \\
$(\mu \mathrm{m})$
\end{tabular} & \begin{tabular}{|l|} 
Bin Mean \\
Diameter \\
$(\mu \mathrm{m})$
\end{tabular} & $\begin{array}{l}\text { Diff. of the } \\
\text { log of the } \\
\text { Size }\end{array}$ & Counts & $\begin{array}{l}\text { Weighted } \\
\text { Mean } \\
\text { Diameter }\end{array}$ \\
\hline $0.30-0.49$ & .38 & 0.21 & 11088 & \\
\hline $0.50-0.99$ & .70 & 0.30 & 5458 & \\
\hline $1.00-1.99$ & 1.41 & 0.30 & 2709 & \\
\hline $2.00-4.99$ & 3.16 & 0.40 & 17549 & \\
\hline $5.00-9.99$ & 7.07 & 0.30 & 14996 & \\
\hline$>10.00$ & 14.14 & 0.30 & 215507 & \\
\hline
\end{tabular}

\begin{tabular}{|c|c|c|c|c|}
\hline \multicolumn{5}{|c|}{ Sample \#4 } \\
\hline $\begin{array}{l}\text { Bin } \\
\text { Range } \\
(\mu \mathrm{m})\end{array}$ & \begin{tabular}{|l} 
Bin Mean \\
Diameter \\
$(\mu \mathrm{m})$
\end{tabular} & $\begin{array}{l}\text { Diff. of the } \\
\log \text { of the } \\
\text { Size }\end{array}$ & Counts & $\begin{array}{l}\text { Weighted } \\
\text { Mean } \\
\text { Diameter }\end{array}$ \\
\hline $0.30-0.49$ & .38 & 0.21 & 16395 & \multirow{6}{*}{3.48} \\
\hline $0.50-0.99$ & .70 & 0.30 & 31960 & \\
\hline $1.00-1.99$ & 1.41 & 0.30 & 30730 & \\
\hline $2.00-4.99$ & 3.16 & 0.40 & 89901 & \\
\hline $5.00-9.99$ & 7.07 & 0.30 & 22728 & \\
\hline$>10.00$ & $\overline{14.14}$ & 0.30 & $\overline{14123}$ & \\
\hline
\end{tabular}

\begin{tabular}{|c|c|c|c|c|}
\hline \multicolumn{5}{|c|}{ Sample \#6 } \\
\hline $\begin{array}{l}\text { Bin } \\
\text { Range } \\
(\mu \mathrm{m})\end{array}$ & \begin{tabular}{|l|} 
Bin Mean \\
Diameter \\
$(\mu \mathrm{m})$
\end{tabular} & $\begin{array}{l}\text { Diff. of the } \\
\log \text { of the } \\
\text { Size }\end{array}$ & Counts & \begin{tabular}{|l} 
Weighted \\
Mean \\
Diameter
\end{tabular} \\
\hline $0.30-0.49$ & .38 & 0.21 & 46823 & \multirow{6}{*}{1.43} \\
\hline $0.50-0.99$ & .70 & 0.30 & 28683 & \\
\hline $1.00-1.99$ & $\overline{1.41}$ & 0.30 & 13354 & \\
\hline $2.00-4.99$ & $\overline{3.16}$ & 0.40 & 21403 & \\
\hline $5.00-9.99$ & 7.07 & 0.30 & 3088 & \\
\hline$>10.00$ & 14.14 & 0.30 & 1262 & \\
\hline
\end{tabular}

\begin{tabular}{|c|c|c|c|c|}
\hline \multicolumn{5}{|c|}{ Sample \#8 } \\
\hline $\begin{array}{l}\text { Bin } \\
\text { Range } \\
(\mu \mathrm{m})\end{array}$ & \begin{tabular}{|l} 
Bin Mean \\
Diameter \\
$(\mu \mathrm{m})$
\end{tabular} & $\begin{array}{l}\text { Diff. of the } \\
\text { log of the } \\
\text { Size }\end{array}$ & Counts & $\begin{array}{l}\text { Weighted } \\
\text { Mean } \\
\text { Diameter }\end{array}$ \\
\hline $0.30-0.49$ & .38 & 0.21 & 36268 & \multirow{6}{*}{1.66} \\
\hline $0.50-0.99$ & .70 & 0.30 & 33544 & \\
\hline $0.00-1.99$ & 1.41 & 0.30 & 18187 & \\
\hline $2.00 \cdot 4.99$ & 3.16 & 0.40 & 29005 & \\
\hline $5.00-9.99$ & 7.07 & 0.30 & 3891 & \\
\hline$>10.00$ & 14.14 & 0.30 & 1448 & \\
\hline
\end{tabular}


Sample \#9

\begin{tabular}{|c|c|c|c|c|}
\hline $\begin{array}{l}\text { Bin } \\
\text { Range } \\
(\mu \mathrm{m})\end{array}$ & $\begin{array}{l}\text { Bin Mean } \\
\text { Diameter } \\
(\mu \mathrm{m})\end{array}$ & $\begin{array}{l}\text { Diff. of the } \\
\text { log of the } \\
\text { Size }\end{array}$ & Counts & $\begin{array}{l}\text { Weighted } \\
\text { Mean } \\
\text { Diameter }\end{array}$ \\
\hline $0.30-0.49$ & 0.38 & 0.21 & 38823 & \multirow{6}{*}{1.54} \\
\hline $0.50-0.99$ & 0.70 & 0.30 & 32809 & \\
\hline $1.00-1.99$ & 1.41 & 0.30 & 16719 & \\
\hline $2.00-4.99$ & 3.16 & 0.40 & 25838 & \\
\hline $5.00-9.99$ & 7.07 & 0.30 & 3290 & \\
\hline$>10.00$ & 14.14 & 0.30 & 1146 & \\
\hline
\end{tabular}

Sample \#11

\begin{tabular}{|c|c|c|c|c|}
\hline $\begin{array}{l}\text { Bin } \\
\text { Range } \\
(\mu \mathrm{m})\end{array}$ & $\begin{array}{l}\text { Bin Mean } \\
\text { Diameter } \\
(\mu \mathrm{m})\end{array}$ & $\begin{array}{l}\text { Diff. of the } \\
\text { log of the } \\
\text { Size }\end{array}$ & Counts & $\begin{array}{l}\text { Weighted } \\
\text { Mean } \\
\text { Diameter }\end{array}$ \\
\hline $0.30 \cdot 0.49$ & 0.38 & 0.21 & 31711 & \multirow{6}{*}{1.79} \\
\hline $0.50-0.99$ & 0.70 & 0.30 & 35649 & \\
\hline $1.00-1.99$ & 1.41 & 0.30 & 21319 & \\
\hline $2.00-4.99$ & 3.16 & 0.40 & 35823 & \\
\hline $5.00-9.99$ & 7.07 & 0.30 & 4579 & \\
\hline$>10.00$ & 14.14 & 0.30 & 1443 & \\
\hline
\end{tabular}

Sample \#13

\begin{tabular}{|c|c|c|c|c|}
\hline $\begin{array}{l}\text { Bin } \\
\text { Range } \\
(\mu \mathrm{m})\end{array}$ & \begin{tabular}{|l|} 
Bin Mean \\
Diameter \\
$(\mu \mathrm{m})$
\end{tabular} & $\begin{array}{l}\text { Diff. of the } \\
\log \text { of the } \\
\text { Size }\end{array}$ & Counts & $\begin{array}{l}\text { Weighted } \\
\text { Mean } \\
\text { Diameter }\end{array}$ \\
\hline $.0 .30-0.49$ & 0.38 & 0.21 & 28804 & \multirow{6}{*}{1.91} \\
\hline $0.50-0.99$ & 0.70 & 0.30 & 37077 & \\
\hline $1.00-1.99$ & 1.41 & 0.30 & 24448 & \\
\hline $2.00-4.99$ & 3.16 & 0.40 & 43401 & \\
\hline $5.00-9.99$ & 7.07 & 0.30 & 5583 & \\
\hline$>10.00$ & 14.14 & 0.30 & 1426 & \\
\hline
\end{tabular}

Samole \#10

\begin{tabular}{|c|c|c|c|c|}
\hline $\begin{array}{l}\text { Bin } \\
\text { Range } \\
(\mu \mathrm{m})\end{array}$ & \begin{tabular}{|l|} 
Bin Moan \\
Diameter \\
$(\mu \mathrm{m})$
\end{tabular} & $\begin{array}{l}\text { Diff. of the } \\
\text { log of the } \\
\text { Size }\end{array}$ & Counts & \begin{tabular}{|l} 
Weighted \\
Mean \\
Diameter
\end{tabular} \\
\hline $0.30-0.49$ & .38 & 0.21 & 34946 & \multirow{6}{*}{1.68} \\
\hline $0.50-0.99$ & .70 & 0.30 & 34677 & \\
\hline $1.00-1.99$ & 1.41 & 0.30 & 19472 & \\
\hline $2.00-4.99$ & 3.16 & 0.40 & 31549 & \\
\hline $5.00-9.99$ & 7.07 & 0.30 & 3988 & \\
\hline$>10.00$ & 14.14 & 0.30 & 1369 & \\
\hline
\end{tabular}

\begin{tabular}{|c|c|c|c|c|}
\hline $\begin{array}{l}\text { Bin } \\
\text { Range } \\
(\mu \mathrm{m})\end{array}$ & \begin{tabular}{|l} 
Bin Mean \\
Diameter \\
( $\mu \mathrm{m})$
\end{tabular} & $\begin{array}{l}\text { Diff. of the } \\
\text { log of the } \\
\text { Size }\end{array}$ & Counts & $\begin{array}{l}\text { Weighted } \\
\text { Mean } \\
\text { Diameter }\end{array}$ \\
\hline $0.30-0.49$ & .38 & 0.21 & 28567 & \multirow{6}{*}{1.92} \\
\hline $0.50-0.99$ & .70 & 0.30 & 36941 & \\
\hline $1.00-1.99$ & 1.41 & 0.30 & 24546 & \\
\hline $2.00-4.99$ & 3.16 & 0.40 & 43639 & \\
\hline $5.00-9.99$ & 7.07 & 0.30 & 5591 & \\
\hline$>10.00$ & 14.14 & 0.30 & 1576 & \\
\hline
\end{tabular}

\begin{tabular}{|c|c|c|c|c|}
\hline $\begin{array}{l}\text { Bin } \\
\text { Range } \\
(\mu \mathrm{m})\end{array}$ & \begin{tabular}{|l|} 
Bin Mean \\
Diameter \\
$(\mu \mathrm{m})$
\end{tabular} & \begin{tabular}{|l} 
Diff. of the \\
$\log$ of the \\
Size
\end{tabular} & Counts & $\begin{array}{l}\text { Weighted } \\
\text { Mean } \\
\text { Diameter }\end{array}$ \\
\hline $0.30-0.49$ & .38 & 0.21 & 26361 & \multirow{6}{*}{2.01} \\
\hline $0.50-0.99$ & .70 & 0.30 & 37687 & \\
\hline $1.00-1.99$ & 1.41 & 0.30 & 27028 & \\
\hline $2.00-4.99$ & 3.16 & 0.40 & 49468 & \\
\hline $5.00-9.99$ & 7.07 & 0.30 & 6494 & \\
\hline$>10.00$ & 14.14 & 0.30 & 1547 & \\
\hline
\end{tabular}




\section{Aerosol Particle Measurement Record}

Description of sample location 3706 A Building, Resuspension Test, at $0.5 \mathrm{~m}$, Date 8/12/94, Time sample started 13:27:22, Temperature 84.7 F, Humidity 37.3\%, Dew Point $55.7 \mathrm{~F}$, Average Wind Velocity (if applicable) $.00 \mathrm{~m} / \mathrm{s}$, \# of phyșical barriers between sample area and outside (if applicable) 1

\begin{tabular}{|c|c|c|c|c|}
\hline $\begin{array}{l}\text { Bin } \\
\text { Range } \\
(\omega \mathrm{m})\end{array}$ & $\begin{array}{l}\text { Bin Mean } \\
\text { Diameter } \\
(\mu \mathrm{m})\end{array}$ & $\begin{array}{l}\text { Diff. of the } \\
\log \text { of the } \\
\text { Size }\end{array}$ & Counts & $\begin{array}{l}\text { Weighted } \\
\text { Mean } \\
\text { Diameter }\end{array}$ \\
\hline $0.30-0.49$ & 0.38 & 0.21 & 44256 & \multirow{6}{*}{0.89} \\
\hline $0.50-0.99$ & 0.70 & 0.30 & 46410 & \\
\hline $1.00-1.99$ & 1.41 & 0.30 & 9612 & \\
\hline $2.00-4.99$ & 3.16 & 0.40 & 9390 & \\
\hline $5.00-9.99$ & 7.07 & 0.30 & 531 & \\
\hline$>10.00$ & 14.14 & 0.30 & 162 & \\
\hline \multicolumn{5}{|l|}{ Sample \#3 } \\
\hline $\begin{array}{l}\text { Bin } \\
\text { Range } \\
(\mu \mathrm{m})\end{array}$ & \begin{tabular}{|l|} 
Bin Mean \\
Diamoter \\
$(\mu \mathrm{m})$
\end{tabular} & \begin{tabular}{|l|} 
Diff. of the \\
log of the \\
Size
\end{tabular} & Counts & $\begin{array}{l}\text { Weighted } \\
\text { Mean } \\
\text { Diameter }\end{array}$ \\
\hline $0.30-0.49$ & 0.38 & 0.21 & 3750 & \\
\hline $0.50-0.99$ & 0.70 & 0.30 & 24629 & \\
\hline $1.00-1.99$ & 1.41 & 0.30 & 25244 & \\
\hline $2.00-4.99$ & 3.16 & 0.40 & 130179 & \\
\hline $5.00-9.99$ & 7.07 & 0.30 & 61599 & \\
\hline$>10.00$ & 14.14 & 0.30 & 74918 & \\
\hline \multicolumn{5}{|l|}{ Sample \#5 } \\
\hline \begin{tabular}{|l} 
Bin \\
Range \\
$(\mu \mathrm{m})$
\end{tabular} & \begin{tabular}{|l|} 
Bin Mean \\
Diameter \\
(um)
\end{tabular} & \begin{tabular}{|l|} 
Diff. of the \\
log of the \\
Size
\end{tabular} & Counts & \begin{tabular}{|l} 
Weighted \\
Mean \\
Diameter
\end{tabular} \\
\hline $0.30-0.49$ & 0.38 & 0.21 & 22645 & \multirow{6}{*}{1.74} \\
\hline $0.50-0.99$ & 0.70 & 0.30 & 63808 & \\
\hline $1.00-1.99$ & 1.41 & 0.30 & 21158 & \\
\hline $2.00-4.99$ & 3.16 & 0.40 & 34389 & \\
\hline $5.00-9.99$ & 7.07 & 0.30 & 3643 & \\
\hline$>10.00$ & 14.14 & 0.30 & 2875 & \\
\hline \multicolumn{5}{|l|}{ Sample \#7 } \\
\hline \begin{tabular}{|l} 
Bin \\
Range \\
$(\mu \mathrm{m})$ \\
\end{tabular} & \begin{tabular}{|l|} 
Bin Mean \\
Diameter \\
$(\mathrm{em})$
\end{tabular} & \begin{tabular}{|l|} 
Diff. of the \\
log of the \\
Size
\end{tabular} & Counts & \begin{tabular}{|l} 
Weighted \\
Mean \\
Diameter
\end{tabular} \\
\hline $0.30 \cdot 0.49$ & 0.38 & 0.21 & 25103 & \multirow{6}{*}{1.52} \\
\hline $0.50-0.99$ & 0.70 & 0.30 & 62862 & \\
\hline $1.00-1.99$ & 1.41 & 0.30 & 23831 & \\
\hline $2.00-4.99$ & 3.16 & 0.40 & 29375 & \\
\hline $5.00-9.99$ & 7.07 & 0.30 & 2578 & \\
\hline$>10.00$ & 14.14 & 0.30 & 1583 & \\
\hline \multicolumn{5}{|l|}{ Sample \#9 } \\
\hline \begin{tabular}{|l} 
Bin \\
Range \\
(um)
\end{tabular} & \begin{tabular}{|l|} 
Bin Mean \\
Diameter \\
$(\mu \mathrm{m})$
\end{tabular} & $\begin{array}{l}\text { Diff. of the } \\
\text { log of the } \\
\text { Size }\end{array}$ & Counts & \begin{tabular}{|l|} 
Weighted \\
Mean \\
Diameter
\end{tabular} \\
\hline $0.30-0.49$ & 0.38 & 0.21 & 21925 & \multirow{6}{*}{1.60} \\
\hline $0.50-0.99$ & 0.70 & 0.30 & 64761 & \\
\hline $1.00-1.99$ & 1.41 & 0.30 & 28152 & \\
\hline $2.00-4.99$ & 3.16 & 0.40 & 36230 & \\
\hline $5.00-9.99$ & 7.07 & 0.30 & 2905 & \\
\hline$>10.00$ & 14.14 & 0.30 & 1454 & \\
\hline
\end{tabular}

Sample \#2

\begin{tabular}{|c|c|c|c|c|}
\hline $\begin{array}{l}\text { Bin } \\
\text { Range } \\
(u \mathrm{~m})\end{array}$ & \begin{tabular}{|l|} 
Bin Mean \\
Diameter \\
$(u m)$
\end{tabular} & $\begin{array}{l}\text { Diff. of the } \\
\text { log of the } \\
\text { Size }\end{array}$ & Counts & $\begin{array}{l}\text { Weighted } \\
\text { Mean } \\
\text { Diameter }\end{array}$ \\
\hline $0.30-0.49$ & .38 & 0.21 & 22060 & \multirow{6}{*}{5.26} \\
\hline $0.50-0.99$ & .70 & 0.30 & 436 & \\
\hline $1.00-1.99$ & 1.41 & 0.30 & 23963 & \\
\hline $2.00-4.99$ & 3.16 & 0.40 & 58229 & \\
\hline $5.00-9.99$ & 7.07 & 0.30 & 12269 & \\
\hline$>10.00$ & 14.14 & 0.30 & 56290 & \\
\hline
\end{tabular}

\begin{tabular}{|c|c|c|c|c|}
\hline \multicolumn{5}{|l|}{ Sample \#4 } \\
\hline $\begin{array}{l}\text { Bin } \\
\text { Range } \\
(\mu \mathrm{m})\end{array}$ & \begin{tabular}{|l} 
Bin Mean \\
Diameter \\
$(\mathrm{(m)})$
\end{tabular} & $\begin{array}{l}\text { Diff. of the } \\
\text { log of the } \\
\text { Size }\end{array}$ & Counts & $\begin{array}{l}\text { Woighted } \\
\text { Mean } \\
\text { Diamoter }\end{array}$ \\
\hline $0.30-0.49$ & .38 & 0.21 & 8050 & \multirow{6}{*}{3.17} \\
\hline $0.50-0.99$ & .70 & 0.30 & 57788 & \\
\hline $1.00-1.99$ & 1.41 & 0.30 & 53451 & \\
\hline $2.00-4.99$ & 3.16 & 0.40 & 148248 & \\
\hline $5.00-9.99$ & 7.07 & 0.30 & 25525 & \\
\hline$>10.00$ & 14.14 & 0.30 & 14714 & \\
\hline
\end{tabular}

Sample \#6

\begin{tabular}{|c|c|c|c|c|}
\hline $\begin{array}{l}\text { Bin } \\
\text { Range } \\
(\mu \mathrm{m})\end{array}$ & $\begin{array}{l}\text { Bin Mean } \\
\text { Diameter } \\
(\mathrm{mm})\end{array}$ & $\begin{array}{l}\text { Diff. of the } \\
\log \text { of the } \\
\text { Size }\end{array}$ & Counts & $\begin{array}{l}\text { Weighted } \\
\text { Mean } \\
\text { Diameter }\end{array}$ \\
\hline $0.30-0.49$ & .38 & 0.21 & 26631 & \multirow{6}{*}{1.50} \\
\hline $0.50-0.99$ & .70 & 0.30 & 60907 & \\
\hline $1.00-1.99$ & 1.41 & 0.30 & 22792 & \\
\hline $2.00-4.99$ & $\overline{3.16}$ & 0.40 & 28460 & \\
\hline $5.00-9.99$ & 7.07 & 0.30 & 2398 & \\
\hline$>10.00$ & 14.14 & 0.30 & 1586 & \\
\hline
\end{tabular}

\begin{tabular}{|c|c|c|c|c|}
\hline \multicolumn{5}{|l|}{ Sample \#8 } \\
\hline $\begin{array}{l}\text { Bin } \\
\text { Range } \\
(\mu \mathrm{m})\end{array}$ & \begin{tabular}{|l|} 
Bin Mean \\
Diameter \\
$(\mu \mathrm{m})$
\end{tabular} & $\begin{array}{l}\text { Diff. of the } \\
\text { log of the } \\
\text { Size }\end{array}$ & Counts & \begin{tabular}{|l} 
Weighted \\
Mean \\
Diamoter
\end{tabular} \\
\hline $0.30-0.49$ & .38 & 0.21 & 27838 & \multirow{6}{*}{1.44} \\
\hline $0.50-0.99$ & .70 & 0.30 & 59093 & \\
\hline $0.00-1.99$ & 1.41 & 0.30 & 20202 & \\
\hline $2.00-4.99$ & 3.16 & 0.40 & $\overline{24159}$ & \\
\hline $5.00-9.99$ & 7.07 & 0.30 & 2152 & \\
\hline$>10.00$ & 14.14 & 0.30 & 1587 & \\
\hline \multicolumn{5}{|c|}{ Sample \#10 } \\
\hline $\begin{array}{l}\text { Bin } \\
\text { Range } \\
\text { (um) }\end{array}$ & $\begin{array}{l}\text { Bin Mean } \\
\text { Diameter } \\
(\mu \mathrm{m})\end{array}$ & $\begin{array}{l}\text { Diff. of the } \\
\text { log of the } \\
\text { Size }\end{array}$ & Counts & $\begin{array}{l}\text { Weighted } \\
\text { Mean } \\
\text { Diameter }\end{array}$ \\
\hline $0.30-0.49$ & .38 & 0.21 & 23091 & \multirow{6}{*}{1.55} \\
\hline $0.50-0.99$ & .70 & 0.30 & 63513 & \\
\hline $1.00-1.99$ & 1.41 & 0.30 & 25688 & \\
\hline $2.00-4.99$ & 3.16 & 0.40 & 33082 & \\
\hline $5.00-9.99$ & 7.07 & 0.30 & 2579 & \\
\hline$>10.00$ & 14.14 & 0.30 & 1379 & \\
\hline
\end{tabular}




\begin{tabular}{|c|c|c|c|c|}
\hline $\begin{array}{l}\text { Bin } \\
\text { Range } \\
(\mu \mathrm{m})\end{array}$ & $\begin{array}{l}\text { Bin Mean } \\
\text { Diameter } \\
(\mu \mathrm{m})\end{array}$ & $\begin{array}{l}\text { Diff. of the } \\
\log \text { of the } \\
\text { Size }\end{array}$ & Counts & $\begin{array}{l}\text { Weighted } \\
\text { Mean } \\
\text { Diameter }\end{array}$ \\
\hline $0.30-0.49$ & 0.38 & 0.21 & 22434 & \multirow{6}{*}{1.54} \\
\hline $0.50-0.99$ & 0.70 & 0.30 & 63351 & \\
\hline $1.00-1.99$ & 1.41 & 0.30 & 26889 & \\
\hline $2.00-4.99$ & 3.16 & 0.40 & 34585 & \\
\hline $5.00-9.99$ & 7.07 & 0.30 & 2630 & \\
\hline$>10.00$ & 14.14 & 0.30 & 1003 & \\
\hline
\end{tabular}

\begin{tabular}{|c|c|c|c|c|}
\hline \multicolumn{5}{|l|}{ Sample \#13 } \\
\hline $\begin{array}{l}\text { Bin } \\
\text { Range } \\
\text { (um) }\end{array}$ & $\begin{array}{l}\text { Bin Mean } \\
\text { Diameter } \\
(\mu \mathrm{m})\end{array}$ & $\begin{array}{l}\text { Diff. of the } \\
\text { log of the } \\
\text { Size }\end{array}$ & Counts & $\begin{array}{l}\text { Weighted } \\
\text { Mean } \\
\text { Diameter }\end{array}$ \\
\hline $0.30-0.49$ & 0.38 & 0.21 & 26379 & \multirow{6}{*}{1.43} \\
\hline $0.50-0.99$ & 0.70 & 0.30 & 58519 & \\
\hline $1.00-1.99$ & 1.41 & 0.30 & 21988 & \\
\hline $2.00-4.99$ & 3.16 & 0.40 & 27748 & \\
\hline $5.00-9.99$ & 7.07 & 0.30 & 2054 & \\
\hline$>10.00$ & 14.14 & 0.30 & 875 & \\
\hline
\end{tabular}

Sample \#12

\begin{tabular}{||c|c|l|r|r|}
\hline $\begin{array}{l}\text { Bin } \\
\text { Range } \\
(\mathrm{mm})\end{array}$ & $\begin{array}{l}\text { Bin Mean } \\
\text { Diameter } \\
(\mathrm{um})\end{array}$ & $\begin{array}{l}\text { Diff. of the } \\
\text { log of the } \\
\text { Size }\end{array}$ & Counts & $\begin{array}{l}\text { Weighted } \\
\text { Mean } \\
\text { Diameter }\end{array}$ \\
\hline $0.30-0.49$ & .38 & 0.21 & 23899 & \\
\hline $0.50-0.99$ & .70 & 0.30 & 62005 & \multirow{2}{*}{1.51} \\
\hline $1.00-1.99$ & 1.41 & 0.30 & 25046 & \\
\hline $2.00-4.99$ & 3.16 & 0.40 & 32352 & \\
\hline $5.00-9.99$ & 7.07 & 0.30 & 2394 \\
\hline$>10.00$ & 14.14 & 0.30 & 976 & \\
\hline
\end{tabular}

Sample \#14

\begin{tabular}{||c|c|c|r|r|}
\hline $\begin{array}{l}\text { Bin } \\
\text { Range } \\
(\mu \mathrm{m})\end{array}$ & $\begin{array}{l}\text { Bin Mean } \\
\text { Diameter } \\
(\mathrm{mm})\end{array}$ & $\begin{array}{l}\text { Diff. of the } \\
\text { log of the } \\
\text { Size }\end{array}$ & Counts & $\begin{array}{l}\text { Weighted } \\
\text { Mean } \\
\text { Diameter }\end{array}$ \\
\hline $0.30-0.49$ & .38 & 0.21 & 22595 & \\
\hline $0.50-0.99$ & .70 & .0 .30 & 61678 & \\
\cline { 1 - 4 } $1.00-1.99$ & 1.41 & 0.30 & 25412 & \multirow{2}{*}{1.63} \\
\hline $2.00-4.99$ & 3.16 & 0.40 & 33033 & \\
\hline $5.00-9.99$ & 7.07 & 0.30 & 2672 & \\
\hline$>10.00$ & 14.14 & 0.30 & 2079 & \\
\hline
\end{tabular}




\section{Aerosol Particle Measurement Record}

Description of sample location 3706 A Building, Resuspension Test, at $25 \mathrm{~cm}$, Date 8/12/94, Time sample started 13:27:22, Temperature 84.7 F, Humidity $37.3 \%$, Dew Point $55.7 \mathrm{~F}$, Average Wind Velocity (if applicable) $.00 \mathrm{~m} / \mathrm{s}$, \# of physical barriers between sample area and outside (if applicable) 1

\begin{tabular}{|c|c|c|c|c|}
\hline $\begin{array}{l}\text { Bin } \\
\text { Range } \\
(u m)\end{array}$ & $\mid \begin{array}{l}\text { Bin Mean } \\
\text { Diameter } \\
(\mu \mathrm{m})\end{array}$ & $\begin{array}{l}\text { Diff. of the } \\
\text { log of the } \\
\text { Size }\end{array}$ & Counts & $\begin{array}{l}\text { Weighted } \\
\text { Mean } \\
\text { Diameter }\end{array}$ \\
\hline $0.30 \cdot 0.49$ & 0.38 & 0.21 & 55630 & \multirow{6}{*}{0.97} \\
\hline $0.50-0.99$ & 0.70 & 0.30 & 28028 & \\
\hline $1.00-1.99$ & 1.41 & 0.30 & 9736 & \\
\hline $2.00-4.99$ & 3.16 & 0.40 & 11790 & \\
\hline $5.00-9.99$ & 7.07 & 0.30 & 1169 & \\
\hline$>10.00$ & 14.14 & 0.30 & 268 & \\
\hline \multicolumn{5}{|l|}{ Sample \#3 } \\
\hline \begin{tabular}{|l} 
Bin \\
Range \\
(em)
\end{tabular} & $\begin{array}{l}\text { Bin Mean } \\
\text { Diameter } \\
\text { (um) }\end{array}$ & $\begin{array}{l}\text { Diff. of the } \\
\log \text { of the } \\
\text { Size }\end{array}$ & Counts & $\begin{array}{l}\text { Weighted } \\
\text { Mean } \\
\text { Diameter }\end{array}$ \\
\hline $0.30-0.49$ & 0.38 & 0.21 & 6456 & \multirow{6}{*}{5.51} \\
\hline $0.50-0.99$ & 0.70 & 0.30 & 18127 & \\
\hline $1.00-1.99$ & 1.41 & 0.30 & 22728 & \\
\hline $2.00-4.99$ & 3.16 & 0.40 & 119987 & \\
\hline $5.00-9.99$ & 7.07 & 0.30 & 55413 & \\
\hline$>10.00$ & 14.14 & 0.30 & 47379 & \\
\hline \multicolumn{5}{|l|}{ Sample $\# 5$} \\
\hline $\begin{array}{l}\text { Bin } \\
\text { Range } \\
(u m)\end{array}$ & \begin{tabular}{|l|} 
Bin Mean \\
Diameter \\
$($ (um)
\end{tabular} & $\begin{array}{l}\text { Diff. of the } \\
\text { log of the } \\
\text { Size }\end{array}$ & Counts & $\begin{array}{l}\text { Weighted } \\
\text { Mean } \\
\text { Diameter }\end{array}$ \\
\hline $0.30 \cdot 0.49$ & 0.38 & 0.21 & 32297 & \multirow{6}{*}{1.95} \\
\hline $0.50-0.99$ & 0.70 & 0.30 & 32603 & \\
\hline $1.00-1.99$ & 1.41 & 0.30 & 20389 & \\
\hline $2.00-4.99$ & 3.16 & 0.40 & 38368 & \\
\hline $5.00-9.99$ & 7.07 & 0.30 & 5918 & \\
\hline$>10.00$ & 14.14 & 0.30 & 2134 & \\
\hline \multicolumn{5}{|l|}{ Sample \#7 } \\
\hline \begin{tabular}{|l|}
$\begin{array}{l}\text { Bin } \\
\text { Range } \\
(\mathrm{m})\end{array}$ \\
\end{tabular} & $\begin{array}{l}\text { Bin Mean } \\
\text { Diameter } \\
(\mu \mathrm{m})\end{array}$ & $\begin{array}{l}\text { Diff. of the } \\
\text { log of the } \\
\text { Size }\end{array}$ & Counts & \begin{tabular}{|l} 
Weighted \\
Mean \\
Diameter
\end{tabular} \\
\hline $0.30 \cdot 0.49$ & 0.38 & 0.21 & 36415 & \multirow{6}{*}{1.64} \\
\hline $0.50 \cdot 0.99$ & 0.70 & 0.30 & 31669 & \\
\hline $1.00-1.99$ & 1.41 & 0.30 & 16888 & \\
\hline $2.00-4.99$ & 3.16 & 0.40 & 27010 & \\
\hline $5.00-9.99$ & 7.07 & 0.30 & 3748 & \\
\hline$>10.00$ & 14.14 & 0.30 & 1407 & \\
\hline \multicolumn{5}{|l|}{ Sample \#9 } \\
\hline $\begin{array}{l}\text { Bin } \\
\text { Range } \\
\text { (um) }\end{array}$ & $\begin{array}{l}\text { Bin Mean } \\
\text { Diameter } \\
\text { (urm) }\end{array}$ & \begin{tabular}{|l|} 
Diff. of the \\
log of the \\
Size
\end{tabular} & Counts & \begin{tabular}{|l|} 
Weighted \\
Mean \\
Diameter
\end{tabular} \\
\hline $0.30 \cdot 0.49$ & 0.38 & 0.21 & 30025 & \multirow{6}{*}{1.85} \\
\hline $0.50 \cdot 0.99$ & 0.70 & 0.30 & 33268 & \\
\hline $1.00-1.99$ & 1.41 & 0.30 & 20474 & \\
\hline $2.00 \cdot 4.99$ & 3.16 & 0.40 & 36382 & \\
\hline $5.00-9.99$ & 7.07 & 0.30 & 5066 & \\
\hline$>10.00$ & 14.14 & 0.30 & 1446 & \\
\hline
\end{tabular}

\begin{tabular}{|c|c|c|c|c|}
\hline $\begin{array}{l}\text { Bin } \\
\text { Range } \\
\text { (um) }\end{array}$ & $\begin{array}{l}\text { Bin Mean } \\
\text { Diameter } \\
\text { (em) }\end{array}$ & $\begin{array}{l}\text { Diff. of the } \\
\log \text { of the } \\
\text { Size }\end{array}$ & Counts & $\begin{array}{l}\text { Weighted } \\
\text { Mean } \\
\text { Diameter }\end{array}$ \\
\hline $0.30-0.49$ & .38 & 0.21 & 12182 & \\
\hline $0.50-0.99$ & .70 & 0.30 & 11130 & \\
\hline $1.00-1.99$ & 1.41 & 0.30 & 8619 & \\
\hline $2.00-4.99$ & 3.16 & 0.40 & 41176 & \\
\hline $5.00-9.99$ & 7.07 & 0.30 & 25857 & \\
\hline$>10.00$ & 14.14 & 0.30 & 137107 & \\
\hline \multicolumn{5}{|l|}{ Sample \#4 } \\
\hline \begin{tabular}{|l|} 
Bin \\
Range \\
(um) \\
\end{tabular} & \begin{tabular}{|l} 
Bin Mean \\
Diameter \\
$(u m)$
\end{tabular} & $\begin{array}{l}\text { Diff. of the } \\
\text { log of the } \\
\text { Size }\end{array}$ & Counts & $\begin{array}{l}\text { Weighted } \\
\text { Mean } \\
\text { Diameter }\end{array}$ \\
\hline $0.30-0.49$ & .38 & 0.21 & 14764 & \multirow{6}{*}{3.77} \\
\hline $0.50-0.99$ & .70 & 0.30 & 27416 & \\
\hline $1.00-1.99$ & 1.41 & 0.30 & 28843 & \\
\hline $2.00-4.99$ & 3.16 & 0.40 & 110639 & \\
\hline $5.00-9.99$ & 7.07 & 0.30 & 34351 & \\
\hline$>10.00$ & 14.14 & 0.30 & 15004 & \\
\hline \multicolumn{5}{|l|}{ Sample \#6 } \\
\hline $\begin{array}{l}\text { Bin } \\
\text { Range } \\
(\mathrm{erm})\end{array}$ & $\begin{array}{l}\text { Bin Mean } \\
\text { Diameter } \\
(\text { (um) }\end{array}$ & $\begin{array}{l}\text { Diff. of the } \\
\text { log of the } \\
\text { Size }\end{array}$ & Counts & $\begin{array}{l}\text { Weighted } \\
\text { Mean } \\
\text { Diameter }\end{array}$ \\
\hline $0.30-0.49$ & .38 & 0.21 & 42279 & \multirow{6}{*}{1.44} \\
\hline $0.50-0.99$ & .70 & 0.30 & 29795 & \\
\hline $1.00-1.99$ & 1.41 & 0.30 & 13606 & \\
\hline $2.00-4.99$ & 3.16 & 0.40 & 20881 & \\
\hline $5.00-9.99$ & 7.07 & 0.30 & 2838 & \\
\hline$>10.00$ & 14.14 & 0.30 & 1254 & \\
\hline \multicolumn{5}{|l|}{ Sample \#8 } \\
\hline $\begin{array}{l}\text { Bin } \\
\text { Range } \\
(u \mathrm{~m})\end{array}$ & $\begin{array}{l}\text { Bin Mean } \\
\text { Diameter } \\
\text { (um) }\end{array}$ & \begin{tabular}{|l|} 
Diff. of the \\
log of the \\
Size
\end{tabular} & Counts & $\begin{array}{l}\text { Weighted } \\
\text { Mean } \\
\text { Diameter }\end{array}$ \\
\hline $0.30-0.49$ & .38 & 0.21 & 35799 & \multirow{6}{*}{1.65} \\
\hline $0.50-0.99$ & .70 & 0.30 & 31365 & \\
\hline $0.00-1.99$ & 1.41 & 0.30 & 16802 & \\
\hline $2.00-4.99$ & 3.16 & 0.40 & 27357 & \\
\hline $5.00-9.99$ & 7.07 & 0.30 & 3696 & \\
\hline$>10.00$ & 14.14 & 0.30 & 1430 & \\
\hline \multicolumn{5}{|c|}{ Sample \#10 } \\
\hline \begin{tabular}{l|} 
Bin \\
Range \\
(um)
\end{tabular} & \begin{tabular}{|l} 
Bin Mean. \\
Diameter \\
$(u m)$
\end{tabular} & $\begin{array}{l}\text { Diff. of the } \\
\text { log of the } \\
\text { Size }\end{array}$ & Counts & $\begin{array}{l}\text { Weighted } \\
\text { Mean } \\
\text { Diameter }\end{array}$ \\
\hline $0.30-0.49$ & .38 & 0.21 & 28349 & \multirow{6}{*}{1.92} \\
\hline $0.50-0.99$ & .70 & 0.30 & 33277 & \\
\hline $1.00-1.99$ & 1.41 & 0.30 & 21941 & \\
\hline $2.00-4.99$ & 3.16 & 0.40 & 39629 & \\
\hline $5.00-9.99$ & 7.07 & 0.30 & 5371 & \\
\hline$>10.00$ & 14.14 & 0.30 & 1509 & \\
\hline
\end{tabular}


Sample \#11

\begin{tabular}{|c|c|c|c|c|}
\hline $\begin{array}{l}\text { Bin } \\
\text { Range } \\
(e m)\end{array}$ & $\begin{array}{l}\text { Bin Mean } \\
\text { Diameter } \\
(\mu \mathrm{m})\end{array}$ & $\begin{array}{l}\text { Diff. of the } \\
\text { log of the } \\
\text { Size }\end{array}$ & Counts & $\begin{array}{l}\text { Weighted } \\
\text { Mean } \\
\text { Diameter }\end{array}$ \\
\hline $0.30-0.49$ & 0.38 & 0.21 & 28729 & \multirow{6}{*}{1.89} \\
\hline $0.50-0.99$ & 0.70 & 0.30 & 33016 & \\
\hline $1.00-1.99$ & 1.41 & 0.30 & 21099 & \\
\hline $2.00-4.99$ & 3.16 & 0.40 & 38313 & \\
\hline $5.00-9.99$ & 7.07 & 0.30 & 5207 & \\
\hline$>10.00$ & 14.14 & 0.30 & 1399 & \\
\hline
\end{tabular}

Sample \#13

\begin{tabular}{|c|c|c|c|c|}
\hline $\begin{array}{l}\text { Bin } \\
\text { Range } \\
(\mu \mathrm{m})\end{array}$ & \begin{tabular}{|l} 
Bin Mean \\
Diameter \\
$(\mu \mathrm{m})$
\end{tabular} & $\begin{array}{l}\text { Diff. of the } \\
\log \text { of the } \\
\text { Size }\end{array}$ & Counts & $\begin{array}{l}\text { Weighted } \\
\text { Mean } \\
\text { Diameter }\end{array}$ \\
\hline $0.30 \cdot 0.49$ & 0.38 & 0.21 & 32020 & \multirow{6}{*}{1.75} \\
\hline $0.50-0.99$ & 0.70 & 0.30 & 31289 & \\
\hline $1.00-1.99$ & 1.41 & 0.30 & 19025 & \\
\hline $2.00-4.99$ & 3.16 & 0.40 & 32989 & \\
\hline $5.00-9.99$ & 7.07 & 0.30 & 4180 & \\
\hline$>10.00$ & 14.14 & 0.30 & 1143 & \\
\hline
\end{tabular}

Samole \#12

\begin{tabular}{|c|c|c|c|c|}
\hline $\begin{array}{l}\text { Bin } \\
\text { Range } \\
(\mu \mathrm{m})\end{array}$ & $\begin{array}{l}\text { Bin Mean } \\
\text { Diameter } \\
(\mu \mathrm{m})\end{array}$ & $\begin{array}{l}\text { Diff. of the } \\
\text { log of the } \\
\text { Size }\end{array}$ & Counts & $\begin{array}{l}\text { Weighted } \\
\text { Mean } \\
\text { Diameter }\end{array}$ \\
\hline $0.30-0.49$ & .38 & 0.21 & 29774 & \multirow{6}{*}{1.86} \\
\hline $0.50-0.99$ & .70 & 0.30 & 32340 & \\
\hline $1.00-1.99$ & 1.41 & 0.30 & 20217 & \\
\hline $2.00-4.99$ & 3.16 & 0.40 & 36536 & \\
\hline $5.00-9.99$ & 7.07 & 0.30 & 5037 & \\
\hline$>10.00$ & 14.14 & 0.30 & 1390 & \\
\hline
\end{tabular}

Sample \#14

\begin{tabular}{||c|c|c|r|r||}
\hline \begin{tabular}{|c|c|r||} 
Bin \\
$(\mu \mathrm{m})$
\end{tabular} & $\begin{array}{l}\text { Bin Mean } \\
\text { Diameter } \\
(\mu \mathrm{m})\end{array}$ & $\begin{array}{l}\text { Diff. of the } \\
\text { log of the } \\
\text { Size }\end{array}$ & Counts & $\begin{array}{l}\text { Weighted } \\
\text { Mean } \\
\text { Diameter }\end{array}$ \\
\hline $0.30-0.49$ & .38 & 0.21 & 33567 & \\
\hline $0.50-0.99$ & .70 & 0.30 & 30672 & \multirow{2}{*}{1.69} \\
\hline $1.00-1.99$ & 1.41 & 0.30 & 17493 & \\
\hline $2.00-4.99$ & 3.16 & 0.40 & 29955 & \\
\hline $5.00-9.99$ & 7.07 & 0.30 & 3946 & \\
\hline$>10.00$ & 14.14 & 0.30 & 1142 & \\
\hline
\end{tabular}


Aerosol Particle Measurement Record

Description of sample location 3706A Building, Resuspension Test, at $1.0 \mathrm{~m}$, Date 8/12/94, Time sample started 8:21:08, Temperature 80.3 F, Humidity $42.5 \%$, Dew Point 55.5 F, Average Wind Velocity (if applicable) $.00 \mathrm{~m} / \mathrm{s}$, \# of physical barriers between sample area and outside (if applicable) 1

\begin{tabular}{|c|c|c|c|c|}
\hline \begin{tabular}{|l} 
Bin \\
Range \\
(um)
\end{tabular} & \begin{tabular}{|l|} 
Bin Mean \\
Diameter \\
(um)
\end{tabular} & $\begin{array}{l}\text { Diff. of the } \\
\log \text { of the } \\
\text { Size }\end{array}$ & Counts & $\begin{array}{l}\text { Weighted } \\
\text { Mean } \\
\text { Diameter }\end{array}$ \\
\hline $0.30-0.49$ & 0.38 & 0.21 & 47783 & \multirow{6}{*}{0.87} \\
\hline $0.50-0.99$ & 0.70 & 0.30 & 52746 & \\
\hline $1.00-1.99$ & 1.41 & 0.30 & 10158 & \\
\hline $2.00-4.99$ & 3.16 & 0.40 & 9704 & \\
\hline $5.00-9.99$ & 7.07 & 0.30 & 463 & \\
\hline$>10.00$ & 14.14 & 0.30 & 98 & \\
\hline \multicolumn{5}{|l|}{ Sample \#3 } \\
\hline \begin{tabular}{|l} 
Bin \\
Range \\
(um)
\end{tabular} & $\begin{array}{l}\text { Bin Mean } \\
\text { Diameter } \\
(\mu \mathrm{m})\end{array}$ & $\begin{array}{l}\text { Diff. of the } \\
\text { log of the } \\
\text { Size }\end{array}$ & Counts & \begin{tabular}{|l} 
Weighted \\
Mean \\
Diameter
\end{tabular} \\
\hline $0.30 \cdot 0.49$ & 0.38 & 0.21 & 47390 & \multirow{6}{*}{0.86} \\
\hline $0.50-0.99$ & 0.70 & 0.30 & 51753 & \\
\hline $1.00-1.99$ & 1.41 & 0.30 & 10209 & \\
\hline $2.00 \cdot 4.99$ & 3.16 & 0.40 & 9364 & \\
\hline $5.00-9.99$ & 7.07 & 0.30 & 427 & \\
\hline$>10.00$ & 14.14 & 0.30 & 99 & \\
\hline \multicolumn{5}{|l|}{ Sample \#5 } \\
\hline \begin{tabular}{|l|} 
Bin \\
Range \\
$(\mu \mathrm{m})$ \\
\end{tabular} & \begin{tabular}{|l} 
Bin Mean \\
Diameter \\
$(u m)$
\end{tabular} & $\begin{array}{l}\text { Diff. of the } \\
\text { log of the } \\
\text { Size }\end{array}$ & Counts & \begin{tabular}{|l} 
Weighted \\
Mean \\
Diameter
\end{tabular} \\
\hline $0.30-0.49$ & 0.38 & 0.21 & 29515 & \multirow{6}{*}{1.38} \\
\hline $0.50-0.99$ & 0.70 & 0.30 & 69782 & \\
\hline $1.00-1.99$ & 1.41 & 0.30 & 24119 & \\
\hline $2.00-4.99$ & 3.16 & 0.40 & 26913 & \\
\hline $5.00-9.99$ & 7.07 & 0.30 & 1768 & \\
\hline$>10,00$ & 14.14 & 0.30 & 1397 & \\
\hline \multicolumn{5}{|l|}{ Sample \#7 } \\
\hline \begin{tabular}{|l} 
Bin \\
Range \\
(um)
\end{tabular} & \begin{tabular}{|l} 
Bin Mean \\
Diameter \\
(um)
\end{tabular} & $\begin{array}{l}\text { Diff. of the } \\
\log \text { of the } \\
\text { Size }\end{array}$ & Counts & $\begin{array}{l}\text { Weighted } \\
\text { Mean } \\
\text { Diameter }\end{array}$ \\
\hline $0.30-0.49$ & 0.38 & 0.21 & 23215 & \multirow{6}{*}{1.58} \\
\hline $0.50-0.99$ & 0.70 & 0.30 & 80160 & \\
\hline $1.00-1.99$ & 1.41 & 0.30 & 34454 & \\
\hline $2.00-4.99$ & 3.16 & 0.40 & 42085 & \\
\hline $5.00-9.99$ & 7.07 & 0.30 & 2988 & \\
\hline$>10.00$ & 14.14 & 0.30 & 1761 & \\
\hline \multicolumn{5}{|l|}{ Sample \#9 } \\
\hline $\begin{array}{l}\text { Bin } \\
\text { Range } \\
\text { (um) }\end{array}$ & $\begin{array}{l}\text { Bin Mean } \\
\text { Diameter } \\
(\mathrm{cm})\end{array}$ & $\begin{array}{l}\text { Diff. of the } \\
\text { log of the } \\
\text { Size }\end{array}$ & Counts & $\begin{array}{l}\text { Weighted } \\
\text { Mean } \\
\text { Diameter }\end{array}$ \\
\hline $0.30 \cdot 0.49$ & 0.38 & 0.21 & 18677 & \multirow{6}{*}{1.78} \\
\hline $0.50 \cdot 0.99$ & 0.70 & 0.30 & 85700 & \\
\hline $1.00-1.99$ & 1.41 & 0.30 & 45430 & \\
\hline $2.00-4.99$ & 3.16 & 0.40 & 61511 & \\
\hline $5.00-9.99$ & 7.07 & 0.30 & 4569 & \\
\hline$>10.00$ & 14.14 & 0.30 & 2076 & \\
\hline
\end{tabular}

Sample \#2

\begin{tabular}{|c|c|c|c|c|}
\hline $\begin{array}{l}\text { Bin } \\
\text { Range } \\
\text { (um) }\end{array}$ & $\begin{array}{l}\text { Bin Mean } \\
\text { Diameter } \\
(\text { um) }\end{array}$ & $\begin{array}{l}\text { Diff. of the } \\
\log \text { of the } \\
\text { Size }\end{array}$ & Counts & $\begin{array}{l}\text { Weighted } \\
\text { Mean } \\
\text { Diameter }\end{array}$ \\
\hline $0.30-0.49$ & .38 & 0.21 & 47255 & \multirow{6}{*}{0.87} \\
\hline $0.50-0.99$ & .70 & 0.30 & 51802 & \\
\hline $1.00-1.99$ & 1.41 & 0.30 & 10439 & \\
\hline $2.00-4.99$ & $\overline{3.16}$ & 0.40 & 9414 & \\
\hline $5.00-9.99$ & 7.07 & 0.30 & 420 & \\
\hline$>10.00$ & 14.14 & 0.30 & 126 & \\
\hline \multicolumn{5}{|l|}{ Sample \#4 } \\
\hline $\begin{array}{l}\text { Bin } \\
\text { Range } \\
(u m)\end{array}$ & \begin{tabular}{|l|} 
Bin Mean \\
Diameter \\
$(\mathrm{um})$
\end{tabular} & $\begin{array}{l}\text { Diff. of the } \\
\text { log of the } \\
\text { Size }\end{array}$ & Counts & $\begin{array}{l}\text { Weighted } \\
\text { Mean } \\
\text { Diamoter }\end{array}$ \\
\hline $0.30-0.49$ & .38 & 0.21 & 47186 & \multirow{6}{*}{0.87} \\
\hline $0.50-0.99$ & .70 & 0.30 & 53045 & \\
\hline $1.00-1.99$ & $\overline{1.41}$ & 0.30 & 10469 & \\
\hline $2.00-4.99$ & 3.16 & 0.40 & 9576 & \\
\hline $5.00-9.99$ & 7.07 & 0.30 & 442 & \\
\hline$>10.00$ & 14.14 & 0.30 & 98 & \\
\hline
\end{tabular}

Sample $\$ 6$

\begin{tabular}{|c|c|c|c|c|}
\hline $\begin{array}{l}\text { Bin } \\
\text { Range } \\
\text { (um) }\end{array}$ & $\begin{array}{l}\text { Bin Mean } \\
\text { Diameter } \\
(u \mathrm{~m})\end{array}$ & $\begin{array}{l}\text { Diff. of the } \\
\text { log of the } \\
\text { Size }\end{array}$ & Counts & $\begin{array}{l}\text { Weighted } \\
\text { Mean } \\
\text { Diameter }\end{array}$ \\
\hline $0.30-0.49$ & .38 & 0.21 & 23122 & \multirow{6}{*}{1.59} \\
\hline $0.50-0.99$ & .70 & 0.30 & 80039 & \\
\hline $1.00-1.99$ & 1.41 & 0.30 & 34005 & \\
\hline $2.00-4.99$ & 3.16 & 0.40 & 41724 & \\
\hline $5.00-9.99$ & 7.07 & 0.30 & 2890 & \\
\hline$>10.00$ & 14.14 & 0.30 & 1857 & \\
\hline
\end{tabular}

\begin{tabular}{|c|c|c|c|c|}
\hline $\begin{array}{l}\text { Bin } \\
\text { Range } \\
\text { (lmm) }\end{array}$ & \begin{tabular}{|l} 
Bin Mean \\
Diameter \\
$(\mathrm{mm})$
\end{tabular} & $\begin{array}{l}\text { Diff. of the } \\
\log \text { of the } \\
\text { Size }\end{array}$ & Counts & $\begin{array}{l}\text { Weighted } \\
\text { Mean } \\
\text { Diameter }\end{array}$ \\
\hline $0.30-0.49$ & .38 & 0.29 & 17499 & \\
\hline $0.50-0.99$ & .70 & 0.30 & 86825 & \\
\hline $0.00-1.99$ & 1.41 & 0.30 & 49549 & \\
\hline $2.00-4.99$ & 3.16 & 0.40 & 70548 & \\
\hline $5.00-9.99$ & 7.07 & 0.30 & 5456 & $\alpha$ \\
\hline$>10.00$ & 14.14 & 0.30 & 2573 & \\
\hline
\end{tabular}

\begin{tabular}{|c|c|c|c|c|}
\hline \multicolumn{5}{|c|}{ Sample $\# 10$} \\
\hline $\begin{array}{l}\text { Bin } \\
\text { Range } \\
(\mu \mathrm{m})\end{array}$ & $\begin{array}{l}\text { Bin Mean } \\
\text { Diameter } \\
(\mathrm{um})\end{array}$ & $\begin{array}{l}\text { Diff. of the } \\
\log \text { of the } \\
\text { Size }\end{array}$ & Counts & $\begin{array}{l}\text { Weighted } \\
\text { Mean } \\
\text { Diamoter }\end{array}$ \\
\hline $0.30-0.49$ & .38 & 0.21 & 19455 & \multirow{6}{*}{1.73} \\
\hline $0.50-0.99$ & .70 & 0.30 & 84918 & \\
\hline $1.00-1.99$ & 1.41 & 0.30 & 44051 & \\
\hline $2.00-4.99$ & 3.16 & 0.40 & 58886 & \\
\hline $5.00-9.99$ & 7.07 & 0.30 & 4121 & \\
\hline$>10.00$ & 14.14 & 0.30 & 1730 & \\
\hline
\end{tabular}




\section{Aerosol Particle Measurement Record}

Description of sample location 3706 A Building, Resuspension Test, at $.25 \mathrm{~cm}$, Date 8/12/94, Time sample started 8:21:08, Temperature $80.3 \mathrm{~F}$, Humidity $42.5 \%$, Dew Point $55.5 \mathrm{~F}$, Average Wind Velocity (if applicable) $.00 \mathrm{~m} / \mathrm{s}$, \# of physical barriers between sample area and outside (if applicable) 1

\begin{tabular}{|c|c|c|c|c|}
\hline $\begin{array}{l}\text { Bin } \\
\text { Range } \\
\text { (um) }\end{array}$ & \begin{tabular}{|l|} 
Bin Mean \\
Diameter \\
$(\mathrm{um})$
\end{tabular} & $\begin{array}{l}\text { Diff. of the } \\
\text { log of the } \\
\text { Size }\end{array}$ & Counts & $\begin{array}{l}\text { Weighted } \\
\text { Mean } \\
\text { Diameter }\end{array}$ \\
\hline $0.30-0.49$ & 0.38 & 0.21 & 59034 & \multirow{6}{*}{0.95} \\
\hline $0.50-0.99$ & 0.70 & 0.30 & 30759 & \\
\hline $1.00-1.99$ & 1.41 & 0.30 & 10257 & \\
\hline $2.00-4.99$ & 3.16 & 0.40 & 11996 & \\
\hline $5.00-9.99$ & 7.07 & 0.30 & 1188 & \\
\hline$>10.00$ & 14.14 & 0.30 & 257 & \\
\hline \multicolumn{5}{|l|}{ Sample $\# 3$} \\
\hline $\begin{array}{l}\text { Bin } \\
\text { Range } \\
\text { (um) }\end{array}$ & $\begin{array}{l}\text { Bin Mean } \\
\text { Diameter } \\
(\mu \mathrm{m})\end{array}$ & $\begin{array}{l}\text { Diff. of the } \\
\log \text { of the } \\
\text { Size }\end{array}$ & Counts & $\begin{array}{l}\text { Weighted } \\
\text { Mean } \\
\text { Diameter }\end{array}$ \\
\hline $0.30-0.49$ & 0.38 & 0.21 & 43438 & \multirow{6}{*}{1.52} \\
\hline $0.50-0.99$ & 0.70 & 0.30 & 29201 & \\
\hline $1.00-1.99$ & 1.41 & 0.30 & 13047 & \\
\hline $2.00-4.99$ & 3.16 & 0.40 & 19455 & \\
\hline $5.00-9.99$ & 7.07 & 0.30 & 3114 & \\
\hline$>10.00$ & 14.14 & 0.30 & 1997 & \\
\hline \multicolumn{5}{|l|}{ Sample \#5 } \\
\hline $\begin{array}{l}\text { Bin } \\
\text { Range } \\
(\mu \mathrm{m}) \\
\end{array}$ & $\begin{array}{l}\text { Bin Mean } \\
\text { Diameter } \\
\text { (um) }\end{array}$ & $\begin{array}{l}\text { Diff. of the } \\
\log \text { of the } \\
\text { Size }\end{array}$ & Counts & $\begin{array}{l}\text { Weighted } \\
\text { Mean } \\
\text { Diameter }\end{array}$ \\
\hline $0.30-0.49$ & 0.38 & 0.21 & 56256 & \multirow{6}{*}{1.06} \\
\hline $0.50-0.99$ & 0.70 & 0.30 & 28919 & \\
\hline $1.00-1.99$ & 1.41 & 0.30 & 10211 & \\
\hline $2.00-4.99$ & 3.16 & 0.40 & 12481 & \\
\hline $5.00-9.99$ & 7.07 & 0.30 & 1640 & \\
\hline$>10.00$ & 14.14 & 0.30 & 711 & \\
\hline \multicolumn{5}{|l|}{ Sample \#7 } \\
\hline $\begin{array}{l}\text { Bin } \\
\text { Range } \\
(\mu \mathrm{m}) \\
\end{array}$ & $\begin{array}{l}\text { Bin Mean } \\
\text { Diameter } \\
(\mu \mathrm{m})\end{array}$ & \begin{tabular}{|l|} 
Diff. of the \\
log of the \\
Size
\end{tabular} & Counts & $\begin{array}{l}\text { Weighted } \\
\text { Mean } \\
\text { Diameter }\end{array}$ \\
\hline $0.30 \cdot 0.49$ & 0.38 & 0.21 & 40905 & \multirow{6}{*}{1.51} \\
\hline $0.50-0.99$ & 0.70 & 0.30 & 33216 & \\
\hline $1.00-1.99$ & 1.41 & 0.30 & 16476 & \\
\hline $2.00-4.99$ & 3.16 & 0.40 & 25407 & \\
\hline $5.00-9.99$ & 7.07 & 0.30 & 3166 & \\
\hline$>10.00$ & 14.14 & 0.30 & 1244 & \\
\hline \multicolumn{5}{|l|}{ Sample \#9 } \\
\hline $\begin{array}{l}\text { Bin } \\
\text { Range } \\
\text { (um) }\end{array}$ & \begin{tabular}{|l} 
Bin Mean \\
Diameter \\
$(\mu \mathrm{m})$
\end{tabular} & $\begin{array}{l}\text { Diff. of the } \\
\log \text { of the } \\
\text { Size }\end{array}$ & Counts & $\begin{array}{l}\text { Weighted } \\
\text { Mean } \\
\text { Diameter }\end{array}$ \\
\hline $0.30-0.49$ & 0.38 & 0.21 & 26960 & \multirow{6}{*}{2.03} \\
\hline $0.50-0.99$ & 0.70 & 0.30 & 37758 & \\
\hline $1.00-1.99$ & 1.41 & 0.30 & 26478 & \\
\hline $2.00-4.99$ & 3.16 & 0.40 & 48968 & \\
\hline $5.00-9.99$ & 7.07 & 0.30 & 6666 & \\
\hline$>10.00$ & 14.14 & 0.30 & 1887 & \\
\hline
\end{tabular}

Sample \#2

\begin{tabular}{|c|c|c|c|c|}
\hline $\begin{array}{l}\text { Bin } \\
\text { Range } \\
\text { (um) }\end{array}$ & \begin{tabular}{|l} 
Bin Mean \\
Diameter \\
$(u m)$
\end{tabular} & $\begin{array}{l}\text { Diff. of the } \\
\text { log of the } \\
\text { Size }\end{array}$ & Counts & $\begin{array}{l}\text { Weighted } \\
\text { Mean } \\
\text { Diameter }\end{array}$ \\
\hline $0.30-0.49$ & .38 & 0.21 & 19485 & \\
\hline $0.50-0.99$ & .70 & 0.30 & 14504 & \\
\hline $1.00-1.99$ & 1.41 & 0.30 & 9898 & \\
\hline $2.00-4.99$ & 3.16 & 0.40 & 42441 & \\
\hline $5.00-9.99$ & 7.07 & 0.30 & 22259 & \\
\hline$>10.00$ & 14.14 & 0.30 & 120385 & \\
\hline
\end{tabular}

\begin{tabular}{|c|c|c|c|c|}
\hline \multicolumn{5}{|l|}{ Sample \#4 } \\
\hline $\begin{array}{l}\text { Bin } \\
\text { Range } \\
\text { (um) }\end{array}$ & $\begin{array}{l}\text { Bin Mean } \\
\text { Diameter } \\
(\text { (um) }\end{array}$ & $\begin{array}{l}\text { Diff. of the } \\
\log \text { of the } \\
\text { Size }\end{array}$ & Counts & $\begin{array}{l}\text { Weighted } \\
\text { Mean } \\
\text { Diameter }\end{array}$ \\
\hline $0.30-0.49$ & .38 & 0.21 & 54655 & \multirow{6}{*}{1.10} \\
\hline $0.50-0.99$ & .70 & 0.30 & 29034 & \\
\hline $1.00-1.99$ & 1.41 & 0.30 & 10214 & \\
\hline $2.00-4.99$ & 3.16 & 0.40 & 13143 & \\
\hline $5.00-9.99$ & 7.07 & 0.30 & 1709 & \\
\hline$>10.00$ & 94.14 & 0.30 & 830 & \\
\hline
\end{tabular}

\begin{tabular}{|c|c|c|c|c|}
\hline \multicolumn{5}{|l|}{ Sample \#6 } \\
\hline $\begin{array}{l}\text { Bin } \\
\text { Range } \\
(u m)\end{array}$ & $\begin{array}{l}\text { Bin Mean } \\
\text { Diameter } \\
\text { (um) }\end{array}$ & $\begin{array}{l}\text { Diff. of the } \\
\log \text { of the } \\
\text { Size }\end{array}$ & Counts & $\begin{array}{l}\text { Weighted } \\
\text { Mean } \\
\text { Diameter }\end{array}$ \\
\hline $0.30-0.49$ & .38 & 0.21 & 43488 & \multirow{6}{*}{1.43} \\
\hline $0.50-0.99$ & .70 & 0.30 & 32573 & \\
\hline $1.00-1.99$ & 1.41 & 0.30 & 15574 & \\
\hline $2.00-4.99$ & $\overline{3.16}$ & 0.40 & 23056 & \\
\hline $5.00-9.99$ & 7.07 & 0.30 & 2862 & \\
\hline$>10.00$ & 14.14 & 0.30 & 1093 & \\
\hline
\end{tabular}

\begin{tabular}{|c|c|c|c|c|}
\hline \multicolumn{5}{|l|}{ Sample \#8 } \\
\hline $\begin{array}{l}\text { Bin } \\
\text { Range } \\
\text { (um) }\end{array}$ & \begin{tabular}{|l} 
Bin Mean \\
Diameter \\
$(u \mathrm{~m})$
\end{tabular} & $\begin{array}{l}\text { Diff. of the } \\
\text { log of the } \\
\text { Size }\end{array}$ & Counts & $\begin{array}{l}\text { Weighted } \\
\text { Moan } \\
\text { Diameter }\end{array}$ \\
\hline $0.30 \cdot 0.49$ & .38 & 0.21 & 32957 & \multirow{6}{*}{1.83} \\
\hline $0.50-0.99$ & .70 & 0.30 & 35532 & \\
\hline $0.00-1.99$ & 1.41 & 0.30 & 21529 & \\
\hline $2.00-4.99$ & 3.16 & 0.40 & 37791 & \\
\hline $5.00-9.99$ & 7.07 & 0.30 & 5105 & \\
\hline$>10.00$ & 14.14 & 0.30 & 1577 & \\
\hline
\end{tabular}
Sample \#10

\begin{tabular}{|c|c|c|c|c|}
\hline $\begin{array}{l}\text { Bin } \\
\text { Range } \\
\text { (um) }\end{array}$ & \begin{tabular}{|l|} 
Bin Mean \\
Diameter \\
(um)
\end{tabular} & $\begin{array}{l}\text { Diff. of the } \\
\log \text { of the } \\
\text { Size }\end{array}$ & Counts & $\begin{array}{l}\text { Woighted } \\
\text { Moan } \\
\text { Diamoter }\end{array}$ \\
\hline $0.30-0.49$ & .38 & 0.21 & 26553 & \multirow{6}{*}{2.06} \\
\hline $0.50-0.99$ & .70 & 0.30 & 37656 & \\
\hline $1.00-1.99$ & 1.41 & 0.30 & 26537 & \\
\hline $2.00-4.99$ & 3.16 & 0.40 & 50191 & \\
\hline $5.00-9.99$ & 7.07 & 0.30 & 6888 & \\
\hline$>10.00$ & 14.14 & 0.30 & 1927 & \\
\hline
\end{tabular}


Sample \#11

\begin{tabular}{|c|c|c|c|c|}
\hline $\begin{array}{l}\text { Bin } \\
\text { Range } \\
(\mu \mathrm{m})\end{array}$ & $\begin{array}{l}\text { Bin Mean } \\
\text { Diameter } \\
(\mu \mathrm{m})\end{array}$ & $\begin{array}{l}\text { Diff. of the } \\
\text { log of the } \\
\text { Size }\end{array}$ & Counts & $\begin{array}{l}\text { Weighted } \\
\text { Mean } \\
\text { Diameter }\end{array}$ \\
\hline $0.30-0.49$ & 0.38 & 0.21 & 27061 & \multirow{6}{*}{2.04} \\
\hline $0.50-0.99$ & 0.70 & 0.30 & 37442 & \\
\hline $1.00 \cdot 1.99$ & 1.41 & 0.30 & 26123 & \\
\hline $2.00-4.99$ & 3.16 & 0.40 & 48774 & \\
\hline $5.00-9.99$ & 7.07 & 0.30 & 6833 & \\
\hline$>10.00$ & 14.14 & 0.30 & 1834 & \\
\hline
\end{tabular}

Sample \#13

\begin{tabular}{|c|c|c|c|c|}
\hline $\begin{array}{l}\text { Bin } \\
\text { Range } \\
\text { (um) }\end{array}$ & \begin{tabular}{|l|} 
Bin Mean \\
Diameter \\
$($ (um)
\end{tabular} & $\begin{array}{l}\text { Diff. of the } \\
\text { log of the } \\
\text { Size }\end{array}$ & Counts & $\begin{array}{l}\text { Weighted } \\
\text { Mean } \\
\text { Diameter }\end{array}$ \\
\hline $0.30 \cdot 0.49$ & 0.38 & 0.21 & 26689 & \multirow{6}{*}{2.02} \\
\hline $0.50-0.99$ & 0.70 & 0.30 & 38006 & \\
\hline $1.00-1.99$ & 1.41 & 0.30 & 26800 & \\
\hline $2.00-4.99$ & 3.16 & 0.40 & 49403 & \\
\hline $5.00-9.99$ & 7.07 & 0.30 & 6513 & \\
\hline$>10.00$ & 14.14 & 0.30 & 1724 & \\
\hline
\end{tabular}

Sample \#12

\begin{tabular}{|c|c|c|c|c|}
\hline $\begin{array}{l}\text { Bin } \\
\text { Range } \\
(\mu \mathrm{m})\end{array}$ & \begin{tabular}{|l|} 
Bin Mean \\
Diameter \\
$(u m)$
\end{tabular} & $\begin{array}{l}\text { Diff. of the } \\
\text { log of the } \\
\text { Size }\end{array}$ & Counts & \begin{tabular}{|l} 
Weighted \\
Mean \\
Diameter
\end{tabular} \\
\hline $0.30-0.49$ & .38 & 0.21 & 27494 & \multirow{6}{*}{1.99} \\
\hline $0.50-0.99$ & .70 & 0.30 & 37406 & \\
\hline $1.00-1.99$ & 1.41 & 0.30 & 25364 & \\
\hline $2.00-4.99$ & 3.16 & 0.40 & 46858 & \\
\hline $5.00-9.99$ & 7.07 & 0.30 & 6234 & \\
\hline$>10.00$ & 14.14 & 0.30 & 1675 & \\
\hline
\end{tabular}

Sample \#14

\begin{tabular}{|c|c|c|c|c|}
\hline $\begin{array}{l}\text { Bin } \\
\text { Range } \\
\text { (um) }\end{array}$ & $\begin{array}{l}\text { Bin Mean } \\
\text { Diameter } \\
(\nu \mathrm{m})\end{array}$ & $\begin{array}{l}\text { Diff. of the } \\
\text { log of the } \\
\text { Size }\end{array}$ & Counts & $\begin{array}{l}\text { Weighted } \\
\text { Mean } \\
\text { Diameter }\end{array}$ \\
\hline $0.30-0.49$ &.$\quad .38$ & 0.21 & 38180 & \multirow{6}{*}{1.59} \\
\hline $0.50-0.99$ & .70 & 0.30 & 34166 & \\
\hline $1.00-1.99$ & 1.41 & 0.30 & 18522 & \\
\hline $2.00-4.99$ & 3.16 & 0.40 & 30249 & \\
\hline $5.00-9.99$ & 7.07 & 0.30 & 3699 & \\
\hline$>10.00$ & 14.14 & 0.30 & 944 & \\
\hline
\end{tabular}




\section{Aerosol Particle Measurement Record}

Description of sample location 3706 A Building, Resuspension Test, at $1.0 \mathrm{~m}$, Date

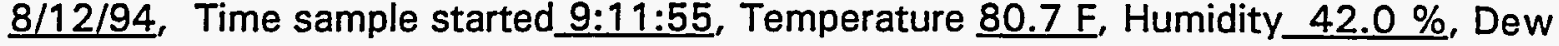
Point $55.8 \mathrm{~F}$, Average Wind Velocity (if applicable) $.00 \mathrm{~m} / \mathrm{s}$, \# of physical barriers between sample area and outside (if applicable) 1

Sample \#1

\begin{tabular}{|c|c|c|c|c|}
\hline $\begin{array}{l}\text { Bin } \\
\text { Range } \\
(\mu \mathrm{m})\end{array}$ & \begin{tabular}{|l} 
Bin Mean \\
Diameter \\
(um)
\end{tabular} & $\begin{array}{l}\text { Diff. of the } \\
\text { log of the } \\
\text { Size }\end{array}$ & Counts & $\begin{array}{l}\text { Weighted } \\
\text { Mean } \\
\text { Diameter }\end{array}$ \\
\hline $0.30-0.49$ & 0.38 & 0.21 & 41780 & \multirow{6}{*}{0.94} \\
\hline $0.50-0.99$ & 0.70 & 0.30 & 51221 & \\
\hline $1.00-1.99$ & 1.41 & 0.30 & 12098 & \\
\hline $2.00-4.99$ & 3.16 & 0.40 & 11288 & \\
\hline $5.00-9.99$ & 7.07 & 0.30 & 514 & \\
\hline$>10.00$ & 14.14 & 0.30 & 95 & \\
\hline
\end{tabular}

\begin{tabular}{|c|c|c|c|c|}
\hline $\begin{array}{l}\text { Bin } \\
\text { Range } \\
\text { (um) }\end{array}$ & $\mid \begin{array}{l}\text { Bin Mean } \\
\text { Diameter } \\
(\mathrm{erm})\end{array}$ & $\begin{array}{l}\text { Diff. of the } \\
\text { log of the } \\
\text { Size }\end{array}$ & Counts & $\begin{array}{l}\text { Weighted } \\
\text { Mean } \\
\text { Diameter }\end{array}$ \\
\hline $0.30-0.49$ & 0.38 & 0.21 & 28320 & \multirow{6}{*}{1.36} \\
\hline $0.50-0.99$ & 0.70 & 0.30 & 75612 & \\
\hline $1.00-1.99$ & 1.41 & 0.30 & 28026 & \\
\hline $2.00-4.99$ & 3.16 & 0.40 & 30205 & \\
\hline $5.00-9.99$ & 7.07 & 0.30 & 1709 & \\
\hline$>10.00$ & 14.14 & 0.30 & 988 & \\
\hline
\end{tabular}

Sample \#5

\begin{tabular}{|c|c|c|c|c|}
\hline $\begin{array}{l}\text { Bin } \\
\text { Range } \\
(\mu \mathrm{m})\end{array}$ & \begin{tabular}{|l} 
Bin Mean \\
Diameter \\
$(\mu \mathrm{m})$
\end{tabular} & $\begin{array}{l}\text { Diff. of the } \\
\log \text { of the } \\
\text { Size }\end{array}$ & Counts & $\begin{array}{l}\text { Weighted } \\
\text { Mean } \\
\text { Diameter }\end{array}$ \\
\hline $0.30-0.49$ & 0.38 & 0.21 & 34724 & \multirow{6}{*}{1.18} \\
\hline $0.50-0.99$ & 0.70 & 0.30 & 58634 & \\
\hline $1.00-1.99$ & 1.41 & 0.30 & 16604 & \\
\hline $2.00-4.99$ & 3.16 & 0.40 & 17588 & \\
\hline $5.00-9.99$ & 7.07 & 0.30 & 1209 & \\
\hline$>10.00$ & 14.14 & 0.30 & 803 & \\
\hline
\end{tabular}

Sample \#7

\begin{tabular}{||l|c|c|r|r||}
\hline $\begin{array}{l}\text { Bin } \\
\text { Range } \\
(\mu \mathrm{m})\end{array}$ & $\begin{array}{l}\text { Bin Mean } \\
\text { Diameter } \\
(\mathrm{mm})\end{array}$ & $\begin{array}{l}\text { Diff. of the } \\
\text { log of the } \\
\text { Size }\end{array}$ & Counts & $\begin{array}{l}\text { Weighted } \\
\text { Mean } \\
\text { Diameter }\end{array}$ \\
\hline $0.30-0.49$ & 0.38 & 0.21 & 25206 & \\
\cline { 1 - 4 } $0.50-0.99$ & 0.70 & 0.30 & 74632 & \multirow{2}{*}{1.48} \\
\hline $1.00-1.99$ & 1.41 & 0.30 & 29554 & \\
\hline $2.00-4.99$ & 3.16 & 0.40 & 34488 & \\
\hline $5.00-9.99$ & 7.07 & 0.30 & 2324 \\
\cline { 1 - 4 } & 14.14 & 0.30 & 1409 & \\
\hline
\end{tabular}

\begin{tabular}{|c|c|c|c|c|}
\hline $\begin{array}{l}\text { Bin } \\
\text { Range } \\
\text { (um) }\end{array}$ & \begin{tabular}{|l} 
Bin Mean \\
Diameter \\
$(\mathrm{sm})$
\end{tabular} & $\begin{array}{l}\text { Diff. of the } \\
\log \text { of the } \\
\text { Size }\end{array}$ & Counts & $\begin{array}{l}\text { Weighted } \\
\text { Mean } \\
\text { Diameter }\end{array}$ \\
\hline $0.30-0.49$ & 0.38 & 0.21 & 21076 & \\
\hline $0.50-0.99$ & 0.70 & 0.30 & 82783 & \\
\hline $1.00-1.99$ & 1.41 & 0.30 & 38970 & \\
\hline $2.00-4.99$ & 3.16 & 0.40 & 49416 & \\
\hline $5.00-9.99$ & 7.07 & 0.30 & 3421 & \\
\hline$>10.00$ & 14.14 & 0.30 & 1841 & \\
\hline
\end{tabular}

Sample \#2

\begin{tabular}{|c|c|c|c|c|}
\hline $\begin{array}{l}\text { Bin } \\
\text { Range } \\
(\mu \mathrm{m}) \\
\end{array}$ & $\begin{array}{l}\text { Bin Mean } \\
\text { Diameter } \\
(\mu \mathrm{m})\end{array}$ & $\begin{array}{l}\text { Diff. of the } \\
\text { log of the } \\
\text { Size }\end{array}$ & Counts & $\begin{array}{l}\text { Weighted } \\
\text { Mean } \\
\text { Diameter }\end{array}$ \\
\hline $0.30-0.49$ & .38 & 0.21 & 43295 & \multirow{6}{*}{0.91} \\
\hline $0.50-0.99$ & .70 & 0.30 & 49929 & \\
\hline $1.00-1.99$ & 1.41 & 0.30 & 10979 & \\
\hline $2.00-4.99$ & 3.16 & 0.40 & 10457 & \\
\hline $5.00-9.99$ & 7.07 & 0.30 & 458 & \\
\hline$>10.00$ & $\overline{14.14}$ & 0.30 & 118 & \\
\hline
\end{tabular}

\begin{tabular}{|c|c|c|c|c|}
\hline \multicolumn{5}{|l|}{ Sample \#4 } \\
\hline $\begin{array}{l}\text { Bin } \\
\text { Range } \\
\text { (um) }\end{array}$ & $\begin{array}{l}\text { Bin Mean } \\
\text { Diameter } \\
(u m)\end{array}$ & $\begin{array}{l}\text { Diff. of the } \\
\text { log of the } \\
\text { Size }\end{array}$ & Counts & $\begin{array}{l}\text { Weighted } \\
\text { Mean } \\
\text { Diameter }\end{array}$ \\
\hline $0.30-0.49$ & .38 & 0.21 & 35187 & \multirow{6}{*}{1.11} \\
\hline $0.50-0.99$ & .70 & 0.30 & 62355 & \\
\hline $1.00-1.99$ & 1.41 & 0.30 & 17963 & \\
\hline $2.00-4.99$ & 3.16 & 0.40 & 17761 & \\
\hline $5.00-9.99$ & 7.07 & 0.30 & 898 & \\
\hline$>10.00$ & 14.14 & 0.30 & 329 & \\
\hline
\end{tabular}

\begin{tabular}{|c|c|c|c|c|}
\hline \multicolumn{5}{|l|}{ Sample $\# 6$} \\
\hline \begin{tabular}{|l} 
Bin \\
Range \\
$(\mu \mathrm{m})$
\end{tabular} & \begin{tabular}{|l} 
Bin Mean \\
Diameter \\
$(\mathrm{um})$
\end{tabular} & $\begin{array}{l}\text { Diff. of the } \\
\text { log of the } \\
\text { Size }\end{array}$ & Counts & $\begin{array}{l}\text { Weighted } \\
\text { Moan } \\
\text { Diameter }\end{array}$ \\
\hline $0.30-0.49$ & .38 & 0.21 & 28644 & \multirow{6}{*}{1.37} \\
\hline $0.50-0.99$ & .70 & 0.30 & 67733 & \\
\hline $1.00-1.99$ & 1.41 & 0.30 & 23054 & \\
\hline $2.00-4.99$ & 3.16 & 0.40 & 25831 & \\
\hline $5.00-9.99$ & 7.07 & 0.30 & 1745 & \\
\hline$>10.00$ & 14.14 & 0.30 & 1255 & \\
\hline
\end{tabular}

\begin{tabular}{|c|c|c|c|c|}
\hline \multicolumn{5}{|l|}{ Sample \#8 } \\
\hline $\begin{array}{l}\text { Bin } \\
\text { Range } \\
(\mu \mathrm{m})\end{array}$ & \begin{tabular}{|l} 
Bin Mean \\
Diameter \\
$(\mu \mathrm{m})$
\end{tabular} & $\begin{array}{l}\text { Diff. of the } \\
\text { log of the } \\
\text { Size }\end{array}$ & Counts & $\begin{array}{l}\text { Weighted } \\
\text { Mean } \\
\text { Diameter }\end{array}$ \\
\hline $0.30-0.49$ & .38 & 0.21 & 33654 & \multirow{6}{*}{1.18} \\
\hline $0.50-0.99$ & .70 & 0.30 & 59991 & \\
\hline $0.00-1.99$ & 1.41 & 0.30 & 17873 & \\
\hline $2.00-4.99$ & 3.16 & 0.40 & 18690 & \\
\hline $5.00-9.99$ & 7.07 & 0.30 & 1157 & \\
\hline$>10.00$ & 14.14 & 0.30 & 635 & \\
\hline
\end{tabular}

\begin{tabular}{|c|c|c|c|c|}
\hline $\begin{array}{l}\text { Bin } \\
\text { Range } \\
\text { (um) }\end{array}$ & \begin{tabular}{|l} 
Bin Mean \\
Diameter \\
$(u m)$
\end{tabular} & $\begin{array}{l}\text { Diff. of the } \\
\log \text { of the } \\
\text { Size }\end{array}$ & Counts & $\begin{array}{l}\text { Weighted } \\
\text { Mean } \\
\text { Diameter }\end{array}$ \\
\hline $0.30-0.49$ & .38 & 0.21 & 23297 & \multirow{6}{*}{1.53} \\
\hline $0.50-0.99$ & .70 & 0.30 & 78616 & \\
\hline $1.00-1.99$ & 1.41 & 0.30 & 33817 & \\
\hline $2.00-4.99$ & 3.16 & 0.40 & 40142 & \\
\hline $5.00-9.99$ & 7.07 & 0.30 & 2697 & \\
\hline$>10.00$ & 14.14 & 0.30 & 1259 & \\
\hline
\end{tabular}


WHC-MR-0496

\begin{tabular}{|c|c|c|c|c|}
\hline $\begin{array}{l}\text { Bin } \\
\text { Range } \\
\text { (um) }\end{array}$ & $\begin{array}{l}\text { Bin Mean } \\
\text { Diameter } \\
(u \mathrm{~m})\end{array}$ & $\begin{array}{l}\text { Diff. of the } \\
\text { log of the } \\
\text { Size }\end{array}$ & Counts & $\begin{array}{l}\text { Weighted } \\
\text { Mean } \\
\text { Diameter }\end{array}$ \\
\hline $0.30-0.49$ & 0.38 & 0.21 & 26173 & \multirow{6}{*}{1.39} \\
\hline $0.50-0.99$ & 0.70 & 0.30 & 72664 & \\
\hline $1.00-1.99$ & 1.41 & 0.30 & 27430 & \\
\hline $2.00-4.99$ & 3.16 & 0.40 & 30713 & \\
\hline $5.00-9.99$ & 7.07 & 0.30 & 1933 & \\
\hline$>10.00$ & 14.14 & 0.30 & 877 & \\
\hline
\end{tabular}

\begin{tabular}{|c|c|c|c|c|}
\hline $\begin{array}{l}\text { Bin } \\
\text { Range } \\
(\mu \mathrm{m})\end{array}$ & $\begin{array}{l}\text { Bin Mean } \\
\text { Diameter } \\
(\text { (um) }\end{array}$ & $\begin{array}{l}\text { Diff. of the } \\
\text { log of the } \\
\text { Size }\end{array}$ & Counts & $\begin{array}{l}\text { Weighted } \\
\text { Mean } \\
\text { Diameter }\end{array}$ \\
\hline $0.30-0.49$ & 0.38 & 0.21 & 22975 & \multirow{6}{*}{1.50} \\
\hline $0.50-0.99$ & 0.70 & 0.30 & 78900 & \\
\hline $1.00-1.99$ & 1.41 & 0.30 & 34026 & \\
\hline $2.00-4.99$ & 3.16 & 0.40 & 40010 & \\
\hline $5.00-9.99$ & 7.07 & 0.30 & 2456 & \\
\hline$>10.00$ & 14.14 & 0.30 & 1002 & \\
\hline
\end{tabular}

Sample \#12

\begin{tabular}{|c|c|c|c|c|}
\hline $\begin{array}{l}\text { Bin } \\
\text { Range } \\
(\mu \mathrm{m})\end{array}$ & $\begin{array}{l}\text { Bin Mean } \\
\text { Diameter } \\
(\mu \mathrm{m})\end{array}$ & $\begin{array}{l}\text { Diff, of the } \\
\text { log of the } \\
\text { Size }\end{array}$ & Counts & $\begin{array}{l}\text { Weighted } \\
\text { Mean } \\
\text { Diameter }\end{array}$ \\
\hline $0.30-0.49$ & .38 & 0.21 & 22935 & \multirow{6}{*}{1.53} \\
\hline $0.50-0.99$ & .70 & 0.30 & 78823 & \\
\hline $1.00-1.99$ & 1.41 & 0.30 & 33597 & \\
\hline $2.00-4.99$ & 3.16 & 0.40 & 40303 & \\
\hline $5.00-9.99$ & 7.07 & 0.30 & 2620 & \\
\hline$>10.00$ & 14.14 & 0.30 & 1192 & \\
\hline
\end{tabular}

\begin{tabular}{|c|c|c|c|c|}
\hline \multicolumn{5}{|c|}{ Samole \#14 } \\
\hline $\begin{array}{l}\text { Bin } \\
\text { Range } \\
\text { (um) }\end{array}$ & \begin{tabular}{|l|} 
Bin Mean \\
Diameter \\
$(u \mathrm{~m})$
\end{tabular} & $\begin{array}{l}\text { Diff. of the } \\
\text { log of the } \\
\text { Size }\end{array}$ & Counts & $\begin{array}{l}\text { Woighted } \\
\text { Mean } \\
\text { Diameter }\end{array}$ \\
\hline $0.30-0.49$ & .38 & 0.21 & 21251 & \multirow{6}{*}{1.59} \\
\hline $0.50-0.99$ & .70 & 0.30 & 82228 & \\
\hline $1.00-1.99$ & 1.41 & 0.30 & $\overline{37943}$ & \\
\hline $2.00-4.99$ & 3.16 & 0.40 & 47069 & \\
\hline $5.00-9.99$ & 7.07 & 0.30 & 3079 & \\
\hline$>10.00$ & 14.14 & 0.30 & 1204 & \\
\hline
\end{tabular}




\section{Aerosol Particle Measurement Record}

Description of sample location 3706 A Building, Resuspension Test, at $25 \mathrm{~cm}$, Date 8/12/94, Time sample started 9:11:55, Temperature $80.7 \mathrm{~F}$, Humidity $42.0 \%$, Dew Point $55.8 \mathrm{~F}$, Average Wind Velocity (if applicable) $.00 \mathrm{~m} / \mathrm{s}$, \# of physical barriers between sample area and outside (if applicable)_1

\begin{tabular}{|c|c|c|c|c|}
\hline $\begin{array}{l}\text { Bin } \\
\text { Range } \\
\text { urml }\end{array}$ & $\begin{array}{l}\text { Bin Mean } \\
\text { Diameter } \\
(\mu \mathrm{m})\end{array}$ & $\begin{array}{l}\text { Diff. of the } \\
\log \text { of the } \\
\text { Size }\end{array}$ & Counts & $\begin{array}{l}\text { Weighted } \\
\text { Mean } \\
\text { Diameter }\end{array}$ \\
\hline $0.30-0.49$ & 0.38 & 0.21 & 59669 & \multirow{6}{*}{0.89} \\
\hline $0.50-0.99$ & 0.70 & 0.30 & 26413 & \\
\hline $1.00-1.99$ & 1.41 & 0.30 & 8490 & \\
\hline $2.00-4.99$ & 3.16 & 0.40 & 9863 & \\
\hline $5.00-9.99$ & 7.07 & 0.30 & 936 & \\
\hline$>10.00$ & 14.14 & 0.30 & 238 & \\
\hline \multicolumn{5}{|l|}{ Sample $\# 3$} \\
\hline \begin{tabular}{|l|} 
Bin \\
Range \\
$(\mu \mathrm{m})$
\end{tabular} & \begin{tabular}{|l} 
Bin Mean \\
Diameter \\
$(\mu \mathrm{m})$
\end{tabular} & $\begin{array}{l}\text { Diff. of the } \\
\log \text { of the } \\
\text { Size }\end{array}$ & Counts & \begin{tabular}{|l} 
Weighted \\
Mean \\
Diameter
\end{tabular} \\
\hline $0,30 \cdot 0.49$ & 0.38 & 0.21 & 35154 & \multirow{6}{*}{1.88} \\
\hline $0.50-0.99$ & 0.70 & 0.30 & 28105 & \\
\hline $1.00-1.99$ & 1.41 & 0.30 & 14439 & \\
\hline $2.00-4.99$ & 3.16 & 0.40 & 25399 & \\
\hline $5.00-9.99$ & 7.07 & 0.30 & 4416 & \\
\hline$>10.00$ & $\overline{14.14}$ & 0.30 & 3029 & \\
\hline \multicolumn{5}{|l|}{ Sample $\# 5$} \\
\hline \begin{tabular}{|l} 
Bin \\
Range \\
(um) \\
\end{tabular} & \begin{tabular}{|l|} 
Bin Mean \\
Diameter \\
$(\mathrm{um})$
\end{tabular} & \begin{tabular}{|l|} 
Diff. of the \\
log of the \\
Size
\end{tabular} & Counts & \begin{tabular}{|l} 
Weighted \\
Mean \\
Diameter
\end{tabular} \\
\hline $0.30-0.49$ & 0.38 & 0.21 & 52602 & \multirow{6}{*}{1.08} \\
\hline $0.50 \cdot 0.99$ & 0.70 & 0.30 & 25609 & \\
\hline $1.00-1.99$ & 1.41 & 0.30 & 8824 & \\
\hline $2,00 \cdot 4.99$ & 3.16 & 0.40 & 11934 & \\
\hline $5.00-9.99$ & 7.07 & 0.30 & 1522 & \\
\hline$>10.00$ & 14.14 & 0.30 & 766 & \\
\hline \multicolumn{5}{|l|}{ Sample \#7 } \\
\hline \begin{tabular}{|l|} 
Bin \\
Range \\
(um)
\end{tabular} & $\begin{array}{l}\text { Bin Mean } \\
\text { Diameter } \\
(\mu \mathrm{m})\end{array}$ & \begin{tabular}{|l|} 
Diff. of the \\
log of the \\
Size
\end{tabular} & Counts & $\begin{array}{l}\text { Weighted } \\
\text { Mean } \\
\text { Diameter }\end{array}$ \\
\hline $0.30 \cdot 0.49$ & 0.38 & 0.21 & 54637 & \multirow{6}{*}{1.02} \\
\hline $0.50-0.99$ & 0.70 & 0.30 & 25872 & \\
\hline $1.00-1.99$ & 1.41 & 0.30 & 8734 & \\
\hline $2.00 \cdot 4.99$ & 3.16 & 0.40 & 11130 & \\
\hline $5.00-9.99$ & 7.07 & 0.30 & 1324 & \\
\hline$>10.00$ & 14.14 & 0.30 & 586 & \\
\hline \multicolumn{5}{|l|}{ Sample \#9 } \\
\hline \begin{tabular}{|l} 
Bin \\
Range \\
$(\mu \mathrm{m})$
\end{tabular} & \begin{tabular}{|l|}
$\begin{array}{l}\text { Bin Mean } \\
\text { Diameter } \\
(u m)\end{array}$ \\
\end{tabular} & \begin{tabular}{|l|} 
Diff. of the \\
log of the \\
Size
\end{tabular} & Counts & \begin{tabular}{|l|} 
Weighted \\
Mean \\
Diameter
\end{tabular} \\
\hline $0.30 \cdot 0.49$ & $\begin{array}{l}0.38 \\
\end{array}$ & 0.21 & 57251 & \multirow{6}{*}{0.93} \\
\hline $0.50-0.99$ & 0.70 & 0.30 & 26017 & \\
\hline $1.00-1.99$ & 1.41 & 0.30 & 8262 & \\
\hline $2.00-4.99$ & 3.16 & 0.40 & 9855 & \\
\hline $5.00-9.99$ & 7.07 & 0.30 & 1052 & \\
\hline$>10.00$ & 14.14 & 0.30 & 416 & \\
\hline
\end{tabular}

Sample \#2

\begin{tabular}{|c|c|c|c|c|}
\hline $\begin{array}{l}\text { Bin } \\
\text { Range } \\
(u m)\end{array}$ & $\begin{array}{l}\text { Bin Mean } \\
\text { Diameter } \\
\text { (um) }\end{array}$ & $\begin{array}{l}\text { Diff. of the } \\
\text { log of the } \\
\text { Size }\end{array}$ & Counts & $\begin{array}{l}\text { Weighted } \\
\text { Mean } \\
\text { Diameter }\end{array}$ \\
\hline $0.30-0.49$ & .38 & 0.21 & 13365 & \\
\hline $0.50-0.99$ & .70 & 0.30 & 7429 & \\
\hline $1.00-1.99$ & 1.41 & 0.30 & 3286 & \\
\hline $2.00-4.99$ & 3.16 & 0.40 & 15316 & \\
\hline $5.00-9.99$ & 7.07 & 0.30 & 13286 & \\
\hline$>10.00$ & 14.14 & 0.30 & 177764 & \\
\hline \multicolumn{5}{|c|}{ Sample \#4 } \\
\hline $\begin{array}{l}\text { Bin } \\
\text { Range } \\
(\mu \mathrm{m})\end{array}$ & $\begin{array}{l}\text { Bin Mean } \\
\text { Diameter } \\
(\mathrm{um})\end{array}$ & $\begin{array}{l}\text { Diff. of the } \\
\text { log of the } \\
\text { Size }\end{array}$ & Counts & $\begin{array}{l}\text { Weighted } \\
\text { Mean } \\
\text { Diameter }\end{array}$ \\
\hline $0.30-0.49$ & .38 & \begin{tabular}{|l|}
0.21 \\
\end{tabular} & 49427 & \multirow{6}{*}{1.22} \\
\hline $0.50-0.99$ & .70 & 0.30 & 25471 & \\
\hline $1.00-1.99$ & 1.41 & 0.30 & 9444 & \\
\hline $2.00-4.99$ & 3.16 & 0.40 & 13221 & \\
\hline $5.00-9.99$ & 7.07 & 0.30 & 2074 & \\
\hline$>10.00$ & 14.14 & 0.30 & 1150 & \\
\hline
\end{tabular}

$$
\text { Sample \#6 }
$$

\begin{tabular}{|c|c|c|c|c|}
\hline $\begin{array}{l}\text { Bin } \\
\text { Range } \\
\text { (um) }\end{array}$ & \begin{tabular}{|l} 
Bin Mean \\
Diameter \\
$(\mathrm{mm})$
\end{tabular} & $\begin{array}{l}\text { Diff. of the } \\
\text { log of the } \\
\text { Size }\end{array}$ & Counts & $\begin{array}{l}\text { Weighted } \\
\text { Mean } \\
\text { Diameter }\end{array}$ \\
\hline $0.30-0.49$ & .38 & 0.21 & 54371 & \multirow{6}{*}{1.03} \\
\hline $0.50-0.99$ & .70 & 0.30 & 26424 & \\
\hline $1.00-1.99$ & 1.41 & 0.30 & $\overline{9018}$ & \\
\hline $2.00-4.99$ & 3.16 & 0.40 & 11499 & \\
\hline $5.00-9.99$ & 7.07 & 0.30 & 1373 & \\
\hline$>10.00$ & 14.14 & 0.30 & 596 & \\
\hline
\end{tabular}

\begin{tabular}{|c|c|c|c|c|}
\hline \multicolumn{5}{|c|}{ Sample \#8 } \\
\hline $\begin{array}{l}\text { Bin } \\
\text { Range } \\
\text { (um) }\end{array}$ & $\begin{array}{l}\text { Bin Mean } \\
\text { Diameter } \\
\text { (um) }\end{array}$ & $\begin{array}{l}\text { Diff. of the } \\
\text { log of the } \\
\text { Size }\end{array}$ & Counts & $\begin{array}{l}\text { Weighted } \\
\text { Mean } \\
\text { Diameter }\end{array}$ \\
\hline $0.30-0.49$ & .38 & 0.21 & 52737 & \multirow{6}{*}{1.08} \\
\hline $0.50-0.99$ & .70 & 0.30 & 26576 & \\
\hline $0.00-1.99$ & 1.41 & 0.30 & 9703 & \\
\hline $2.00-4.99$ & 3.16 & 0.40 & 12607 & \\
\hline $5.00-9.99$ & 7.07 & 0.30 & 1559 & \\
\hline$>10.00$ & 14.14 & 0.30 & 605 & \\
\hline
\end{tabular}

\begin{tabular}{|c|c|c|c|c|}
\hline \multicolumn{5}{|c|}{ Sample $\# 10$} \\
\hline $\begin{array}{l}\text { Bin } \\
\text { Range } \\
(\mathrm{um})\end{array}$ & $\begin{array}{l}\text { Bin Mean } \\
\text { Diameter } \\
(\text { (um) }\end{array}$ & $\begin{array}{l}\text { Diff. of the } \\
\log \text { of the } \\
\text { Size }\end{array}$ & Counts & $\begin{array}{l}\text { Weighted } \\
\text { Mean } \\
\text { Diameter }\end{array}$ \\
\hline $0.30-0.49$ & .38 & 0.21 & 52548 & \multirow{6}{*}{1.06} \\
\hline $0.50-0.99$ & .70 & 0.30 & 27108 & \\
\hline $1.00-1.99$ & 1.41 & 0.30 & 9899 & \\
\hline $2.00-4.99$ & 3.16 & 0.40 & 12475 & \\
\hline $5.00-9.99$ & 7.07 & 0.30 & 1492 & \\
\hline$>10.00$ & 14.14 & 0.30 & 551 & \\
\hline
\end{tabular}


Sample \#11

\begin{tabular}{|c|c|c|c|c|}
\hline $\begin{array}{l}\text { Bin } \\
\text { Range } \\
(\mu \mathrm{m})\end{array}$ & \begin{tabular}{|l} 
Bin Mean \\
Diameter \\
$(\mu \mathrm{m})$
\end{tabular} & $\begin{array}{l}\text { Diff. of the } \\
\text { log of the } \\
\text { Size }\end{array}$ & Counts & \begin{tabular}{|l} 
Weighted \\
Mean \\
Diameter
\end{tabular} \\
\hline $0.30-0.49$ & 0.38 & 0.21 & 49107 & \multirow{6}{*}{1.15} \\
\hline $0.50-0.99$ & 0.70 & 0.30 & 28205 & \\
\hline $1.00-1.99$ & 1.41 & 0.30 & 10928 & \\
\hline $2.00-4.99$ & 3.16 & 0.40 & 14901 & \\
\hline $5.00-9.99$ & 7.07 & 0.30 & 1797 & \\
\hline$>10.00$ & 14.14 & 0.30 & 566 & \\
\hline
\end{tabular}

Sample \#13

\begin{tabular}{|c|c|c|c|c|}
\hline $\begin{array}{l}\text { Bin } \\
\text { Range } \\
(\mu \mathrm{m})\end{array}$ & $\begin{array}{l}\text { Bin Mean } \\
\text { Diameter } \\
\text { (urm) }\end{array}$ & $\begin{array}{l}\text { Diff. of the } \\
\text { log of the } \\
\text { Size }\end{array}$ & Counts & $\begin{array}{l}\text { Weighted } \\
\text { Mean . } \\
\text { Diameter }\end{array}$ \\
\hline $0.30-0.49$ & 0.38 & 0.21 & 41926 & \multirow{6}{*}{1.38} \\
\hline $0.50-0.99$ & 0.70 & 0.30 & 30454 & \\
\hline $1.00-1.99$ & 1.41 & 0.30 & 14554 & \\
\hline $2.00-4.99$ & 3.16 & 0.40 & 21411 & \\
\hline $5.00-9.99$ & 7.07 & 0.30 & 2575 & \\
\hline$>10.00$ & $\overline{14.14}$ & 0.30 & 715 & \\
\hline
\end{tabular}

:
Sample \#12

\begin{tabular}{|c|c|c|c|c|}
\hline $\begin{array}{l}\text { Bin } \\
\text { Range } \\
\text { (um) }\end{array}$ & \begin{tabular}{|l|} 
Bin Mean \\
Diameter \\
$(\mu \mathrm{m})$
\end{tabular} & $\begin{array}{l}\text { Diff. of the } \\
\text { log of the } \\
\text { Size }\end{array}$ & Counts & $\begin{array}{l}\text { Weighted } \\
\text { Mean } \\
\text { Diameter }\end{array}$ \\
\hline $0.30-0.49$ & .38 & 0.21 & 45886 & \multirow{6}{*}{1.26} \\
\hline $0.50-0.99$ & .70 & 0.30 & 28767 & \\
\hline $1.00-1.99$ & 1.41 & 0.30 & 12609 & \\
\hline $2.00-4.99$ & 3.16 & 0.40 & 17832 & \\
\hline $5.00-9.99$ & 7.07 & 0.30 & 2045 & \\
\hline$>10.00$ & 14.14 & 0.30 & 726 & \\
\hline
\end{tabular}

\begin{tabular}{|c|c|c|c|c|}
\hline \multicolumn{5}{|c|}{ Sample \#14 } \\
\hline $\begin{array}{l}\text { Bin } \\
\text { Range } \\
\text { (um) }\end{array}$ & $\begin{array}{l}\text { Bin Mean } \\
\text { Diameter } \\
(\text { (rm) }\end{array}$ & $\begin{array}{l}\text { Diff. of the } \\
\text { log of the } \\
\text { Size }\end{array}$ & Counts & $\begin{array}{l}\text { Weighted } \\
\text { Mean } \\
\text { Diameter }\end{array}$ \\
\hline $0.30-0.49$ & .38 & 0.21 & 37708 & \multirow{6}{*}{1.52} \\
\hline $0.50-0.99$ & .70 & 0.30 & 32133 & \\
\hline $1.00-1.99$ & 1.41 & 0.30 & $\overline{17031}$ & \\
\hline $2.00-4.99$ & 3.16 & 0.40 & 25659 & \\
\hline $5.00-9.99$ & 7.07 & 0.30 & 3060 & \\
\hline$>10.00$ & 14.14 & 0.30 & 945 & \\
\hline
\end{tabular}




\section{Aerosol Particle Measurement Record}

Description of sample location 3706 A Building, Resuspension Test, at $1.0 \mathrm{~m}$, Date 8/12/94, Time sample started 10:04:02, Temperature 81.7 F, Humidity $40.0 \%$, Dew Point 55.4 F, Average Wind Velocity (if applicable) .01 m/s. .\# of physical barriers between sample area and outside (if applicable) 1

Sample \#1

\begin{tabular}{|c|c|c|c|c|}
\hline $\begin{array}{l}\text { Bin } \\
\text { Range } \\
(\mu \mathrm{m})\end{array}$ & $\begin{array}{l}\text { Bin Mean } \\
\text { Diameter } \\
(\mu \mathrm{m})\end{array}$ & $\begin{array}{l}\text { Diff. of the } \\
\log \text { of the } \\
\text { Size }\end{array}$ & Counts & $\begin{array}{l}\text { Weighted } \\
\text { Mean } \\
\text { Diameter }\end{array}$ \\
\hline $0.30-0.49$ & 0.38 & 0.21 & 37922 & \multirow{6}{*}{0.98} \\
\hline $0.50-0.99$ & 0.70 & 0.30 & 50529 & \\
\hline $1.00-1.99$ & 1.41 & 0.30 & 12415 & \\
\hline $2.00 \cdot 4.99$ & 3.16 & 0.40 & 11966 & \\
\hline $5.00-9.99$ & 7.07 & 0.30 & 563 & \\
\hline$>10.00$ & 14.14 & 0.30 & 129 & \\
\hline \multicolumn{5}{|l|}{ Sample \#3 } \\
\hline \begin{tabular}{|l} 
Bin \\
Range \\
$(\mu \mathrm{m})$
\end{tabular} & $\begin{array}{l}\text { Bin Mean } \\
\text { Diameter } \\
(\mu \mathrm{m})\end{array}$ & $\begin{array}{l}\text { Diff. of the } \\
\text { log of the } \\
\text { Size }\end{array}$ & Counts & $\begin{array}{l}\text { Weighted } \\
\text { Mean } \\
\text { Diameter } \\
\end{array}$ \\
\hline $0.30-0.49$ & 0.38 & 0.21 & 34825 & \multirow{6}{*}{1.13} \\
\hline $0.50-0.99$ & 0.70 & 0.30 & 56289 & \\
\hline $1.00-1.99$ & 1.41 & 0.30 & 16790 & \\
\hline $2.00-4.99$ & 3.16 & 0.40 & 17221 & \\
\hline $5.00-9.99$ & 7.07 & 0.30 & 1032 & \\
\hline$>10.00$ & 14.14 & 0.30 & 354 & \\
\hline \multicolumn{5}{|l|}{ Sample \#5 } \\
\hline $\begin{array}{l}\text { Bin } \\
\text { Range } \\
(\mu \mathrm{m}) \\
\end{array}$ & $\begin{array}{l}\text { Bin Mean } \\
\text { Diameter } \\
(\mu \mathrm{m})\end{array}$ & $\begin{array}{l}\text { Diff. of the } \\
\log \text { of the } \\
\text { Size }\end{array}$ & Counts & $\begin{array}{l}\text { Weighted } \\
\text { Mean } \\
\text { Diameter }\end{array}$ \\
\hline $0.30-0.49$ & 0.38 & 0.21 & 24758 & \multirow{6}{*}{1.47} \\
\hline $0.50-0.99$ & 0.70 & 0.30 & 79455 & \\
\hline $1.00-1.99$ & 1.41 & 0.30 & 31405 & \\
\hline $2.00-4.99$ & 3.16 & 0.40 & 35439 & \\
\hline $5.00 \cdot 9.99$ & 7.07 & 0.30 & 2367 & \\
\hline$>10.00$ & 14.14 & 0.30 & 1335 & \\
\hline \multicolumn{5}{|l|}{ Sample \#7 } \\
\hline $\begin{array}{l}\text { Bin } \\
\text { Range } \\
(\mu \mathrm{m}) \\
\end{array}$ & $\begin{array}{l}\text { Bin Mean } \\
\text { Diameter } \\
(\mu \mathrm{m})\end{array}$ & $\begin{array}{l}\text { Diff. of the } \\
\text { log of the } \\
\text { Size }\end{array}$ & Counts. & $\begin{array}{l}\text { Weighted } \\
\text { Mean } \\
\text { Diameter }\end{array}$ \\
\hline $0.30-0.49$ & 0.38 & 0.21 & 20609 & \\
\hline $0.50-0.99$ & 0.70 & 0.30 & 84927 & \\
\hline $1.00-1.99$ & 1.41 & 0.30 & 40178 & \\
\hline $2.00-4.99$ & 3.16 & 0.40 & 50458 & \\
\hline $5.00-9.99$ & 7.07 & 0.30 & 3639 & \\
\hline$>10.00$ & 14.14 & 0.30 & 1893 & \\
\hline \multicolumn{5}{|l|}{ Sample \#9 } \\
\hline $\begin{array}{l}\text { Bin } \\
\text { Range } \\
\text { (um) } \\
\end{array}$ & $\begin{array}{l}\text { Bin Mean } \\
\text { Diameter } \\
(\mu \mathrm{m})\end{array}$ & $\begin{array}{l}\text { Diff. of the } \\
\text { log of the } \\
\text { Size }\end{array}$ & Counts & $\begin{array}{l}\text { Weighted } \\
\text { Mean } \\
\text { Diameter }\end{array}$ \\
\hline $0.30-0.49$ & 0.38 & 0.21 & 25172 & \multirow{6}{*}{1.42} \\
\hline $0.50-0.99$ & 0.70 & 0.30 & 75450 & \\
\hline $1.00-1.99$ & 1.41 & 0.30 & 29381 & \\
\hline $2.00-4.99$ & 3.16 & 0.40 & 33150 & \\
\hline $5.00-9.99$ & 7.07 & 0.30 & 2095 & \\
\hline$>10.00$ & 14.14 & 0.30 & 828 & \\
\hline
\end{tabular}

Sample \#2

\begin{tabular}{|c|c|c|c|c|}
\hline $\begin{array}{l}\text { Bin } \\
\text { Range } \\
(\mu \mathrm{m})\end{array}$ & $\begin{array}{l}\text { Bin Mean } \\
\text { Diameter } \\
(\text { erm) }\end{array}$ & $\begin{array}{l}\text { Diff. of the } \\
\text { log of the } \\
\text { Size }\end{array}$ & Counts & $\begin{array}{l}\text { Weighted } \\
\text { Mean } \\
\text { Diameter }\end{array}$ \\
\hline $0.30-0.49$ & .38 & 0.21 & 35992 & \multirow{6}{*}{1.18} \\
\hline $0.50-0.99$ & .70 & 0.30 & 53685 & \\
\hline $1.00-1.99$ & 1.41 & 0.30 & 16330 & \\
\hline $2.00-4.99$ & 3.16 & 0.40 & 18836 & \\
\hline $5.00-9.99$ & 7.07 & 0.30 & 1248 & \\
\hline$>10.00$ & 14.14 & 0.30 & 477 & \\
\hline
\end{tabular}
Sample \#4

\begin{tabular}{|c|c|c|c|c|}
\hline $\begin{array}{l}\text { Bin } \\
\text { Range } \\
\text { (um) }\end{array}$ & $\begin{array}{l}\text { Bin Mean } \\
\text { Diameter } \\
(\mu \mathrm{m})\end{array}$ & $\begin{array}{l}\text { Diff. of the } \\
\text { log of the } \\
\text { Size }\end{array}$ & Counts & $\begin{array}{l}\text { Weighted } \\
\text { Mean } \\
\text { Diameter }\end{array}$ \\
\hline $0.30-0.49$ & .38 & 0.21 & 22541 & \multirow{6}{*}{1.57} \\
\hline $0.50-0.99$ & .70 & 0.30 & 84207 & \\
\hline $1.00-1.99$ & 1.41 & 0.30 & 37760 & \\
\hline $2.00-4.99$ & 3.16 & 0.40 & 45133 & \\
\hline $5.00-9.99$ & 7.07 & 0.30 & 3033 & \\
\hline$>10.00$ & 14.14 & 0.30 & 1425 & \\
\hline
\end{tabular}
Sample \#6

\begin{tabular}{||c|c|c|r|r||}
\hline $\begin{array}{l}\text { Bin } \\
\text { Range } \\
(\mu \mathrm{m})\end{array}$ & $\begin{array}{l}\text { Bin Mean } \\
\text { Diameter } \\
(\mu \mathrm{m})\end{array}$ & $\begin{array}{l}\text { Diff. of the } \\
\text { log of the } \\
\text { Size }\end{array}$ & Counts & $\begin{array}{l}\text { Weighted } \\
\text { Mean } \\
\text { Diameter }\end{array}$ \\
\hline $0.30-0.49$ & .38 & 0.21 & 24788 & \\
\cline { 1 - 4 } $0.50-0.99$ & .70 & 0.30 & 76395 & \multirow{2}{*}{1.49} \\
\cline { 1 - 4 } $1.00-1.99$ & 1.41 & 0.30 & 30096 & \\
\hline $2.00-4.99$ & 3.16 & 0.40 & 34466 & \\
\hline $5.00-9.99$ & 7.07 & 0.30 & 2503 & \\
\hline$>10.00$ & 14.14 & 0.30 & 1469 & \\
\hline
\end{tabular}

Sample \#8

\begin{tabular}{|c|c|c|c|c|}
\hline $\begin{array}{l}\text { Bin } \\
\text { Range } \\
(\mu \mathrm{m})\end{array}$ & $\begin{array}{l}\text { Bin Mean } \\
\text { Diameter } \\
(\mu \mathrm{m})\end{array}$ & $\begin{array}{l}\text { Diff. of the } \\
\text { log of the } \\
\text { Size }\end{array}$ & Counts & $\begin{array}{l}\text { Weighted } \\
\text { Mean } \\
\text { Diameter }\end{array}$ \\
\hline $0.30-0.49$ & .38 & 0.21 & 22756 & \multirow{6}{*}{1.54} \\
\hline $0.50-0.99$ & .70 & 0.30 & 81261 & \\
\hline $0.00-1.99$ & 1.41 & 0.30 & 34711 & \\
\hline $2.00-4.99$ & 3.16 & 0.40 & 41139 & \\
\hline $5.00-9.99$ & 7.07 & 0.30 & 2808 & \\
\hline$>10.00$ & 14.14 & 0.30 & 1276 & \\
\hline
\end{tabular}

\begin{tabular}{|c|c|c|c|c|}
\hline \multicolumn{5}{|c|}{ Sample \#10 } \\
\hline $\begin{array}{l}\text { Bin } \\
\text { Range } \\
(\mu \mathrm{m})\end{array}$ & $\begin{array}{l}\text { Bin Mean } \\
\text { Diameter } \\
(\mu \mathrm{m})\end{array}$ & $\begin{array}{l}\text { Diff. of the } \\
\text { log of the } \\
\text { Size }\end{array}$ & Counts & $\begin{array}{l}\text { Weighted } \\
\text { Mean } \\
\text { Diameter }\end{array}$ \\
\hline $0.30-0.49$ & .38 & 0.21 & 24941 & \multirow{6}{*}{1.41} \\
\hline $0.50-0.99$ & .70 & 0.30 & 75630 & \\
\hline $1.00-1.99$ & 1.41 & 0.30 & 29389 & \\
\hline $2.00-4.99$ & 3.16 & 0.40 & 33396 & \\
\hline $5.00-9.99$ & 7.07 & 0.30 & 1990 & \\
\hline$>10.00$ & 14.14 & 0.30 & 777 & \\
\hline
\end{tabular}


Sample \#11

\begin{tabular}{|c|c|c|c|c|}
\hline $\begin{array}{l}\text { Bin } \\
\text { Range } \\
\text { (ym) }\end{array}$ & $\begin{array}{l}\text { Bin Mean } \\
\text { Diameter } \\
(\mu \mathrm{m})\end{array}$ & $\begin{array}{l}\text { Diff. of the } \\
\text { log of the } \\
\text { Size }\end{array}$ & Counts & $\begin{array}{l}\text { Weighted } \\
\text { Mean } \\
\text { Diameter }\end{array}$ \\
\hline $0.30-0.49$ & 0.38 & 0.21 & 28528 & \multirow{6}{*}{1.30} \\
\hline $0.50-0.99$ & 0.70 & 0.30 & 69485 & \\
\hline $1.00-1.99$ & 1.41 & 0.30 & 23663 & \\
\hline $2.00-4.99$ & 3.16 & 0.40 & 25887 & \\
\hline $5.00-9.99$ & 7.07 & 0.30 & 1600 & \\
\hline$>10.00$ & 14.14 & 0.30 & 620 & \\
\hline \multicolumn{5}{|l|}{ Sample \#13 } \\
\hline $\begin{array}{l}\text { Bin } \\
\text { Range } \\
(\mu \mathrm{m})\end{array}$ & $\begin{array}{l}\text { Bin Mean } \\
\text { Diameter } \\
(\mu \mathrm{m})\end{array}$ & $\begin{array}{l}\text { Diff. of the } \\
\text { log of the } \\
\text { Size }\end{array}$ & Counts & $\begin{array}{l}\text { Weighted } \\
\text { Mean } \\
\text { Diameter }\end{array}$ \\
\hline $0.30-0.49$ & 0.38 & 0.21 & 29466 & \multirow{6}{*}{1.26} \\
\hline $0.50-0.99$ & 0.70 & 0.30 & 66840 & \\
\hline $1.00-1.99$ & 1.41 & 0.30 & 22228 & \\
\hline $2.00-4.99$ & 3.16 & 0.40 & 23480 & \\
\hline $5.00-9.99$ & 7.07 & 0.30 & 1478 & \\
\hline$>10.00$ & 14.14 & 0.30 & 537 & \\
\hline
\end{tabular}

Sample \#12

\begin{tabular}{|c|c|c|c|c|}
\hline $\begin{array}{l}\text { Bin } \\
\text { Range } \\
(\mu \mathrm{m})\end{array}$ & $\begin{array}{l}\text { Bin Mean } \\
\text { Diameter } \\
(\mu \mathrm{m})\end{array}$ & $\begin{array}{l}\text { Diff. of the } \\
\text { log of the } \\
\text { Size }\end{array}$ & Counts & $\begin{array}{l}\text { Weighted } \\
\text { Mean } \\
\text { Diameter }\end{array}$ \\
\hline $0.30-0.49$ & .38 & 0.21 & 27840 & \multirow{6}{*}{1.31} \\
\hline $0.50-0.99$ & .70 & 0.30 & 68944 & \\
\hline $1.00-1.99$ & 1.41 & 0.30 & 23849 & \\
\hline $2.00-4.99$ & 3.16 & 0.40 & 25956 & \\
\hline $5.00-9.99$ & 7.07 & 0.30 & 1588 & \\
\hline$>10.00$ & 14.14 & 0.30 & 646 & \\
\hline
\end{tabular}

$$
\text { Sample \#14 }
$$

\begin{tabular}{|c|c|c|c|c|}
\hline \begin{tabular}{|l} 
Bin \\
Range \\
(em)
\end{tabular} & $\begin{array}{l}\text { Bin Mean } \\
\text { Diameter } \\
(u m)\end{array}$ & $\begin{array}{l}\text { Diff. of the } \\
\log \text { of the } \\
\text { Size }\end{array}$ & Counts & $\begin{array}{l}\text { Weighted } \\
\text { Mean } \\
\text { Diameter }\end{array}$ \\
\hline $0.30-0.49$ & .38 & 0.21 & 32525 & \multirow{6}{*}{1.15} \\
\hline $0.50-0.99$ & .70 & 0.30 & 58829 & \\
\hline $1.00-1.99$ & 1.41 & 0.30 & 17199 & \\
\hline $2.00-4.99$ & 3.16 & 0.40 & 18055 & \\
\hline $5.00-9.99$ & 7.07 & 0.30 & 1088 & \\
\hline$>10.00$ & 14.14 & 0.30 & 340 & \\
\hline
\end{tabular}




\section{Aerosol Particle Measurement Record}

Description of sample location 3706 A Building, Resuspension Test, at 25 $\underline{\mathrm{cm}}$, Date 8/12/94, Time sample started 10:04:02, Temperature 81.7F, Humidity 40.0 $\%$, Dew Point $55.4 \mathrm{~F}$, Average Wind Velocity (if applicable) $.01 \mathrm{~m} / \mathrm{s}$, \# of physical barriers between sample area and outside (if applicable) 1

Sample \#1

\begin{tabular}{|c|c|c|c|c|}
\hline $\begin{array}{l}\text { Bin } \\
\text { Range } \\
(\mathrm{ym})\end{array}$ & \begin{tabular}{|l|} 
Bin Mean \\
Diameter \\
$(\mu \mathrm{m})$
\end{tabular} & $\begin{array}{l}\text { Diff. of the } \\
\text { log of the } \\
\text { Size }\end{array}$ & Counts & $\begin{array}{l}\text { Weighted } \\
\text { Mean } \\
\text { Diameter }\end{array}$ \\
\hline $0.30-0.49$ & 0.38 & 0.21 & 52286 & \multirow{6}{*}{0.99} \\
\hline $0.50-0.99$ & 0.70 & 0.30 & 25051 & \\
\hline $1.00-1.99$ & 1.41 & 0.30 & 8873 & \\
\hline $2.00-4.99$ & 3.16 & 0.40 & 11059 & \\
\hline $5.00-9.99$ & 7.07 & 0.30 & 1119 & \\
\hline$>10.00$ & 14.14 & 0.30 & 338 & \\
\hline \multicolumn{5}{|l|}{ Sample $\# 3$} \\
\hline \begin{tabular}{|l} 
Bin \\
Range \\
$(\mu \mathrm{m})$ \\
\end{tabular} & \begin{tabular}{|l|} 
Bin Mean \\
Diameter \\
(um)
\end{tabular} & \begin{tabular}{|l|} 
Diff. of the \\
log of the \\
Size
\end{tabular} & Counts & \begin{tabular}{|l} 
Weighted \\
Mean \\
Diameter
\end{tabular} \\
\hline $0.30-0.49$ & 0.38 & 0.21 & 10483 & \multirow{6}{*}{4.28} \\
\hline $0.50-0.99$ & 0.70 & 0.30 & 24755 & \\
\hline $1.00-1.99$ & 1.41 & 0.30 & 28079 & \\
\hline $2.00-4.99$ & 3.16 & 0.40 & 123407 & \\
\hline $5.00-9.99$ & 7.07 & 0.30 & 45361 & \\
\hline$>10.00$ & 14.14 & 0.30 & 22372 & \\
\hline \multicolumn{5}{|l|}{ Sample \#5 } \\
\hline $\begin{array}{l}\text { Bin } \\
\text { Range } \\
(u \mathrm{~m}) \\
\end{array}$ & \begin{tabular}{|l|} 
Bin Mean \\
Diameter \\
$(u m)$
\end{tabular} & $\begin{array}{l}\text { Diff. of the } \\
\text { log of the } \\
\text { Size }\end{array}$ & Counts & \begin{tabular}{|l|} 
Weighted \\
Mean \\
Diameter
\end{tabular} \\
\hline $0.30-0.49$ & 0.38 & 0.21 & 36417 & \multirow{6}{*}{1.62} \\
\hline $0.50 \cdot 0.99$ & 0.70 & 0.30 & 31309 & \\
\hline $1.00-1.99$ & 1.41 & 0.30 & 16285 & \\
\hline $2.00 \cdot 4.99$ & 3.16 & 0.40 & 25349 & \\
\hline $5,00 \cdot 9.99$ & 7.07 & 0.30 & 3400 & \\
\hline$>10.00$ & 14.14 & 0.30 & 1570 & \\
\hline
\end{tabular}

Sample \#7

\begin{tabular}{|c|c|c|c|c|}
\hline $\begin{array}{l}\text { Bin } \\
\text { Range } \\
\text { (um) }\end{array}$ & \begin{tabular}{|l|} 
Bin Mean \\
Diameter \\
$(\mathrm{km})$
\end{tabular} & $\begin{array}{l}\text { Diff. of the } \\
\text { log of the } \\
\text { Size }\end{array}$ & Counts & \begin{tabular}{|l} 
Weighted \\
Mean \\
Diameter
\end{tabular} \\
\hline $0.30 \cdot 0.49$ & 0.38 & 0.21 & 43138 & \multirow{6}{*}{1.30} \\
\hline $0.50-0.99$ & 0.70 & 0.30 & 27994 & \\
\hline $1.00-1.99$ & 1.41 & 0.30 & 12348 & \\
\hline $2.00-4.99$ & 3.16 & 0.40 & 16856 & \\
\hline $5.00-9.99$ & 7.07 & 0.30 & 2099 & \\
\hline$>10.00$ & 14.14 & 0.30 & 890 & \\
\hline
\end{tabular}

Sample \#9

\begin{tabular}{|c|c|c|c|c|}
\hline $\begin{array}{l}\text { Bin } \\
\text { Range } \\
(\mu \mathrm{m})\end{array}$ & \begin{tabular}{|l} 
Bin Mean \\
Diameter \\
$(\mathrm{um})$
\end{tabular} & $\begin{array}{l}\text { Diff. of the } \\
\log \text { of the } \\
\text { Size }\end{array}$ & Counts & $\begin{array}{l}\text { Weighted } \\
\text { Mean } \\
\text { Diameter }\end{array}$ \\
\hline $0.30-0.49$ & 0.38 & 0.21 & 39637 & \multirow{6}{*}{1.43} \\
\hline $0.50 \cdot 0.99$ & 0.70 & 0.30 & 30001 & \\
\hline $1.00-1.99$ & 1.41 & 0.30 & 14560 & \\
\hline $2.00 \cdot 4.99$ & 3.16 & 0.40 & 21039 & \\
\hline $5.00-9.99$ & 7.07 & 0.30 & 2619 & \\
\hline$>10.00$ & $\overline{14.14}$ & 0.30 & 1036 & \\
\hline
\end{tabular}

Sample \#2

\begin{tabular}{|c|c|c|c|c|}
\hline \begin{tabular}{|l} 
Bin \\
Range \\
$(u m)$
\end{tabular} & \begin{tabular}{|l|} 
Bin Mean \\
Diameter \\
$(\mathrm{um})$
\end{tabular} & $\begin{array}{l}\text { Diff. of the } \\
\text { log of the } \\
\text { Size }\end{array}$ & Counts & $\begin{array}{l}\text { Weighted } \\
\text { Mean } \\
\text { Diameter }\end{array}$ \\
\hline $0.30-0.49$ & .38 & 0.21 & 5103 & \\
\hline $0.50-0.99$ & .70 & 0.30 & 4663 & \\
\hline $1.00-1.99$ & 1.41 & 0.30 & 3335 & \\
\hline $2.00-4.99$ & 3.16 & 0.40 & 18705 & \\
\hline $5.00-9.99$ & 7.07 & 0.30 & 15246 & \\
\hline$>10.00$ & 14.14 & 0.30 & 207782 & \\
\hline \multicolumn{5}{|c|}{ Sample \#4 } \\
\hline $\begin{array}{l}\text { Bin } \\
\text { Range } \\
(\mu \mathrm{m})\end{array}$ & $\begin{array}{l}\text { Bin Mean } \\
\text { Diameter } \\
(\mu \mathrm{m})\end{array}$ & $\begin{array}{l}\text { Diff. of the } \\
\text { log of the } \\
\text { Size }\end{array}$ & Counts & $\begin{array}{l}\text { Weighted } \\
\text { Mean } \\
\text { Diameter }\end{array}$ \\
\hline $0.30-0.49$ & .38 & 0.21 & 9349 & \multirow{6}{*}{4.28} \\
\hline $0.50-0.99$ & .70 & 0.30 & 25170 & \\
\hline $1.00-1.99$ & 1.41 & 0.30 & 30869 & \\
\hline $2.00-4.99$ & 3.16 & 0.40 & 128489 & \\
\hline $5.00-9.99$ & 7.07 & 0.30 & 43183 & \\
\hline$>10.00$ & 14.14 & 0.30 & 24230 & \\
\hline \multicolumn{5}{|l|}{ Sample \#6 } \\
\hline $\begin{array}{l}\text { Bin } \\
\text { Range } \\
(u \mathrm{~m})\end{array}$ & $\begin{array}{l}\text { Bin Mean } \\
\text { Diameter } \\
(\text { (um) }\end{array}$ & $\begin{array}{l}\text { Diff. of the } \\
\text { log of the } \\
\text { Size }\end{array}$ & Counts & \begin{tabular}{|l|} 
Weighted \\
Mean \\
Diamoter
\end{tabular} \\
\hline $0.30-0.49$ & .38 & 0.21 & 36429 & \multirow{6}{*}{1.60} \\
\hline $0.50-0.99$ & .70 & 0.30 & 31107 & \\
\hline $1.00-1.99$ & 1.41 & 0.30 & 16010 & \\
\hline $2.00-4.99$ & 3.16 & 0.40 & 24484 & \\
\hline $5.00-9.99$ & 7.07 & 0.30 & 3241 & \\
\hline$>10.00$ & 14.14 & 0.30 & 1550 & \\
\hline
\end{tabular}

\begin{tabular}{|c|c|c|c|c|}
\hline \multicolumn{5}{|l|}{ Sample \#8 } \\
\hline \begin{tabular}{|l|} 
Bin \\
Range \\
(um)
\end{tabular} & \begin{tabular}{|l|} 
Bin Mean \\
Diameter \\
(um)
\end{tabular} & $\begin{array}{l}\text { Diff. of the } \\
\text { log of the } \\
\text { Size }\end{array}$ & Counts & $\begin{array}{l}\text { Weighted } \\
\text { Mean } \\
\text { Diameter }\end{array}$ \\
\hline $0.30-0.49$ & .38 & 0.21 & 40826 & \multirow{6}{*}{1.37} \\
\hline $0.50-0.99$ & .70 & 0.30 & 29513 & \\
\hline $0.00-1.99$ & 1.41 & 0.30 & 13890 & \\
\hline $2.00-4.99$ & 3.16 & 0.40 & 19470 & \\
\hline $5.00-9.99$ & 7.07 & 0.30 & 2440 & \\
\hline$>10.00$ & 14.14 & 0.30 & 853 & \\
\hline
\end{tabular}

\begin{tabular}{|c|c|c|c|c|}
\hline \multicolumn{5}{|c|}{ Sample \#10 } \\
\hline $\begin{array}{l}\text { Bin } \\
\text { Range } \\
(\mu \mathrm{m})\end{array}$ & \begin{tabular}{|l} 
Bin Mean \\
Diameter \\
$(\mu \mathrm{m})$
\end{tabular} & $\begin{array}{l}\text { Diff. of the } \\
\text { log of the } \\
\text { Size }\end{array}$ & Counts & $\begin{array}{l}\text { Weighted } \\
\text { Mean } \\
\text { Diameter }\end{array}$ \\
\hline $0.30-0.49$ & .38 & 0.21 & 40381 & \multirow{6}{*}{1.39} \\
\hline $0.50-0.99$ & .70 & 0.30 & 29501 & \\
\hline $1.00-1.99$ & $\overline{1.41}$ & 0.30 & 14016 & \\
\hline $2.00-4.99$ & 3.16 & 0.40 & 20304 & \\
\hline $5.00-9.99$ & 7.07 & 0.30 & 2436 & \\
\hline$>10.00$ & $\overline{14.14}$ & 0.30 & 875 & \\
\hline
\end{tabular}


Sample \#11

\begin{tabular}{|c|c|c|c|c|}
\hline $\begin{array}{l}\text { Bin } \\
\text { Range } \\
(\text { (rm) }\end{array}$ & \begin{tabular}{|l} 
Bin Mean \\
Diameter \\
$(\mu \mathrm{m})$
\end{tabular} & $\begin{array}{l}\text { Diff. of the } \\
\log \text { of the } \\
\text { Size }\end{array}$ & Counts & $\begin{array}{l}\text { Weighted } \\
\text { Mean } \\
\text { Diameter }\end{array}$ \\
\hline $0.30-0.49$ & 0.38 & 0.21 & 41076 & \multirow{6}{*}{1.38} \\
\hline $0.50 \cdot 0.99$ & 0.70 & 0.30 & 29304 & \\
\hline $1.00-1.99$ & 1.41 & 0.30 & 14087 & \\
\hline $2.00 \cdot 4.99$ & 3.16 & 0.40 & 20149 & \\
\hline $5.00-9.99$ & 7.07 & 0.30 & 2323 & \\
\hline$>10.00$ & 14.14 & 0.30 & 923 & \\
\hline
\end{tabular}

\begin{tabular}{|c|c|c|c|c|}
\hline \multicolumn{5}{|l|}{ Sample \#13 } \\
\hline $\begin{array}{l}\text { Bin } \\
\text { Range } \\
\text { (um) }\end{array}$ & \begin{tabular}{|l|} 
Bin Mean \\
Diameter \\
$(\mathrm{vm})$
\end{tabular} & $\begin{array}{l}\text { Diff. of the } \\
\text { log of the } \\
\text { Size }\end{array}$ & Counts & $\begin{array}{l}\text { Weighted } \\
\text { Mean } \\
\text { Diameter }\end{array}$ \\
\hline $0.30 \cdot 0.49$ & 0.38 & 0.21 & 43428 & \multirow{6}{*}{1.27} \\
\hline $0.50-0.99$ & 0.70 & 0.30 & 27879 & \\
\hline $1.00-1.99$ & 1.41 & 0.30 & 12182 & \\
\hline $2.00-4.99$ & 3.16 & 0.40 & 17205 & \\
\hline $5.00-9.99$ & 7.07 & 0.30 & 2065 & \\
\hline$>10.00$ & 14.14 & 0.30 & 624 & \\
\hline
\end{tabular}

Sample \#12

\begin{tabular}{|c|c|c|c|c|}
\hline $\begin{array}{l}\text { Bin } \\
\text { Range } \\
(\mu \mathrm{m})\end{array}$ & \begin{tabular}{|l} 
Bin Mean \\
Diameter \\
$(u m)$
\end{tabular} & $\begin{array}{l}\text { Diff. of the } \\
\text { log of the } \\
\text { Size }\end{array}$ & Counts & $\begin{array}{l}\text { Weighted } \\
\text { Mean } \\
\text { Diameter }\end{array}$ \\
\hline $0.30-0.49$ & .38 & 0.21 & 40239 & \multirow{6}{*}{1.39} \\
\hline $0.50-0.99$ & .70 & 0.30 & 30014 & \\
\hline $1.00-1.99$ & 1.41 & 0.30 & 14342 & \\
\hline $2.00-4.99$ & 3.16 & 0.40 & 20577 & \\
\hline $5.00-9.99$ & 7.07 & 0.30 & 2503 & \\
\hline$>10.00$ & 14.14 & 0.30 & 818 & \\
\hline
\end{tabular}

\begin{tabular}{|c|c|c|c|c|}
\hline \multicolumn{5}{|c|}{ Sample \#14 } \\
\hline $\begin{array}{l}\text { Bin } \\
\text { Range } \\
\text { (um) }\end{array}$ & $\begin{array}{l}\text { Bin Mean } \\
\text { Diameter } \\
(\mathrm{um})\end{array}$ & $\begin{array}{l}\text { Diff. of the } \\
\log \text { of the } \\
\text { Size }\end{array}$ & Counts & $\begin{array}{l}\text { Weighted } \\
\text { Mean } \\
\text { Diameter }\end{array}$ \\
\hline $0.30-0.49$ & .38 & 0.21 & 39846 & \multirow{6}{*}{1.40} \\
\hline $0.50-0.99$ & .70 & 0.30 & 29916 & \\
\hline $1.00-1.99$ & 1.41 & 0.30 & 14313 & \\
\hline $2.00-4.99$ & 3.16 & 0.40 & 21040 & \\
\hline $5.00-9.99$ & 7.07 & 0.30 & 2452 & \\
\hline$>10.00$ & 14.14 & 0.30 & 850 & \\
\hline
\end{tabular}




\section{Aerosol Particle Measurement Record}

Description of sample location 3706A Building, Resuspension Test, at $1.5 \mathrm{~m}$, Date 8/11/94, Time sample started 9:03:59, Temperature78.4 F, Humidity $40.1 \%$, Dew Point $51.9 \mathrm{~F}$, Average Wind Velocity (if applicable) $.01 \mathrm{~m} / \mathrm{s}$, \# of physical barriers between sample area and outside (if applicable) 1

\begin{tabular}{|c|c|c|c|c|}
\hline \begin{tabular}{|l|} 
Bin \\
Range \\
(um)
\end{tabular} & \begin{tabular}{|l|} 
Bin Mean \\
Diameter \\
$(\mu \mathrm{m})$
\end{tabular} & \begin{tabular}{|l|} 
Diff. of the \\
$\log$ of the \\
Size
\end{tabular} & Counts & $\begin{array}{l}\text { Weighted } \\
\text { Mean } \\
\text { Diameter }\end{array}$ \\
\hline $0.30-0.49$ & 0.38 & 0.21 & 51325 & \multirow{6}{*}{0.77} \\
\hline $0.50-0.99$ & 0.70 & 0.30 & 48944 & \\
\hline $1.00-1.99$ & 1.41 & 0.30 & 7455 & \\
\hline $2.00-4.99$ & 3.16 & 0.40 & 6201 & \\
\hline $5.00-9.99$ & 7.07 & 0.30 & 316 & \\
\hline$>10.00$ & 14.14 & 0.30 & 98 & \\
\hline \multicolumn{5}{|l|}{ Sample \#3 } \\
\hline \begin{tabular}{|l} 
Bin \\
Range \\
$(u m)$ \\
\end{tabular} & $\begin{array}{l}\text { Bin Mean } \\
\text { Diameter } \\
(u m)\end{array}$ & \begin{tabular}{|l|} 
Diff. of the \\
log of the \\
Size
\end{tabular} & Counts & $\begin{array}{l}\text { Weighted } \\
\text { Mean } \\
\text { Diameter }\end{array}$ \\
\hline $0.30-0.49$ & 0.38 & 0.21 & 48236 & \multirow{6}{*}{0.83} \\
\hline $0.50-0.99$ & 0.70 & 0.30 & 51416 & \\
\hline $1.00-1.99$ & 1.41 & 0.30 & 8923 & \\
\hline $2.00-4.99$ & 3.16 & 0.40 & 7829 & \\
\hline $5.00-9.99$ & 7.07 & 0.30 & 393 & \\
\hline$>10.00$ & 14.14 & 0.30 & 229 & \\
\hline \multicolumn{5}{|l|}{ sample \#5 } \\
\hline \begin{tabular}{|l|} 
Bin \\
Range \\
$(\mu \mathrm{m})$ \\
\end{tabular} & \begin{tabular}{|l} 
Bin Mean \\
Diameter \\
$(\mathrm{um})$
\end{tabular} & \begin{tabular}{|l|} 
Diff. of the \\
log of the \\
Size
\end{tabular} & Counts & $\begin{array}{l}\text { Weighted } \\
\text { Mean } \\
\text { Diameter }\end{array}$ \\
\hline $0.30 \cdot 0.49$ & 0.38 & 0.21 & 29065 & \multirow{6}{*}{1.41} \\
\hline $0.50 \cdot 0.99$ & 0.70 & 0.30 & 72409 & \\
\hline $1.00-1.99$ & 1.41 & 0.30 & 23740 & \\
\hline $2.00 \cdot 4.99$ & 3.16 & 0.40 & 26786 & \\
\hline $5.00-9.99$ & 7.07 & 0.30 & 2198 & \\
\hline$>10.00$ & 14.14 & 0.30 & 1782 & \\
\hline \multicolumn{5}{|l|}{ Sample \#7 } \\
\hline $\begin{array}{l}\text { Bin } \\
\text { Range } \\
(\mu \mathrm{m}) \\
\end{array}$ & \begin{tabular}{|l|} 
Bin Mean \\
Diameter \\
(um)
\end{tabular} & \begin{tabular}{|l|} 
Diff. of the \\
log of the \\
Size
\end{tabular} & Counts & $\begin{array}{l}\text { Weighted } \\
\text { Mean } \\
\text { Diameter }\end{array}$ \\
\hline $0.30-0.49$ & 0.38 & 0.21 & 24698 & \\
\hline $0.50 \cdot 0.99$ & 0.70 & 0.30 & 77884 & \\
\hline $1.00-1.99$ & 1.41 & 0.30 & 30811 & \\
\hline $2.00-4.99$ & 3.16 & 0.40 & 36942 & \\
\hline $5.00-9.99$ & 7.07 & 0.30 & 2801 & \\
\hline$>10.00$ & 14.14 & 0.30 & 1736 & \\
\hline \multicolumn{5}{|l|}{ Sample \#9 } \\
\hline \begin{tabular}{|l} 
Bin \\
Range \\
$(\mu \mathrm{m})$
\end{tabular} & $\begin{array}{l}\text { Bin Mean } \\
\text { Diameter } \\
(\mu \mathrm{m})\end{array}$ & \begin{tabular}{|l|} 
Diff. of the \\
log of the \\
Size
\end{tabular} & Counts & $\begin{array}{l}\text { Weighted } \\
\text { Mean } \\
\text { Diameter } \\
\end{array}$ \\
\hline $0.30-0.49$ & 0.38 & 0.21 & 26992 & \multirow{6}{*}{1.45} \\
\hline $0.50-0.99$ & 0.70 & 0.30 & 74318 & \\
\hline $1.00-1.99$ & 1.41 & 0.30 & 26317 & \\
\hline $2.00 \cdot 4.99$ & 3.16 & 0.40 & 31609 & \\
\hline $5.00-9.99$ & 7.07 & 0.30 & 2360 & \\
\hline$>10.00$ & 14.14 & 0.30 & 1429 & \\
\hline
\end{tabular}

Sample \#2

\begin{tabular}{|c|c|c|c|c|}
\hline $\begin{array}{l}\text { Bin } \\
\text { Range } \\
(u m)\end{array}$ & \begin{tabular}{|l} 
Bin Mean \\
Diameter \\
$(\mu \mathrm{m})$
\end{tabular} & $\begin{array}{l}\text { Diff. of the } \\
\log \text { of the } \\
\text { Size }\end{array}$ & Counts & $\begin{array}{l}\text { Weighted } \\
\text { Mean } \\
\text { Diameter }\end{array}$ \\
\hline $0.30-0.49$ & .38 & 0.21 & 52072 & \multirow{6}{*}{0.76} \\
\hline $0.50-0.99$ & .70 & 0.30 & 48916 & \\
\hline $1.00-1.99$ & 1.41 & 0.30 & 7435 & \\
\hline $2.00-4.99$ & 3.16 & 0.40 & 6040 & \\
\hline $5.00-9.99$ & 7.07 & 0.30 & 252 & \\
\hline$>10.00$ & 14.14 & 0.30 & 119 & \\
\hline
\end{tabular}

\begin{tabular}{|c|c|c|c|c|}
\hline \multicolumn{5}{|l|}{ Sample \#4 } \\
\hline $\begin{array}{l}\text { Bin } \\
\text { Range } \\
\text { (um) }\end{array}$ & \begin{tabular}{|l} 
Bin Mean \\
Diameter \\
$(u m)$
\end{tabular} & $\begin{array}{l}\text { Diff. of the } \\
\log \text { of the } \\
\text { Size }\end{array}$ & Counts & $\begin{array}{l}\text { Weighted } \\
\text { Mean } \\
\text { Diameter }\end{array}$ \\
\hline $0.30-0.49$ & .38 & 0.21 & 41976 & \multirow{6}{*}{1.04} \\
\hline $0.50-0.99$ & .70 & 0.30 & 58018 & \\
\hline $1.00-1.99$ & 1.41 & 0.30 & 13150 & \\
\hline $2.00-4.99$ & 3.16 & 0.40 & 12941 & \\
\hline $5.00-9.99$ & 7.07 & 0.30 & 903 & \\
\hline$>10.00$ & 14.14 & 0.30 & 775 & \\
\hline
\end{tabular}

Sample \#6

\begin{tabular}{|c|c|c|c|c|}
\hline $\begin{array}{l}\text { Bin } \\
\text { Range } \\
\text { (um) }\end{array}$ & $\begin{array}{l}\text { Bin Mean } \\
\text { Diameter } \\
(\mu \mathrm{m})\end{array}$ & $\begin{array}{l}\text { Diff. of the } \\
\log \text { of the } \\
\text { Size }\end{array}$ & Counts & $\begin{array}{l}\text { Weighted } \\
\text { Mean } \\
\text { Diameter }\end{array}$ \\
\hline $0.30-0.49$ & .38 & 0.21 & 32883 & \multirow{6}{*}{1.26} \\
\hline $0.50-0.99$ & .70 & 0.30 & 67278 & \\
\hline $1.00-1.99$ & 1.41 & 0.30 & 19706 & \\
\hline $2.00-4.99$ & 3.16 & 0.40 & 21368 & \\
\hline $5.00-9.99$ & 7.07 & 0.30 & 1574 & \\
\hline$>10.00$ & 14.14 & 0.30 & 1084 & \\
\hline
\end{tabular}

\begin{tabular}{|c|c|c|c|c|}
\hline \multicolumn{5}{|c|}{ Sample $\# 8$} \\
\hline \begin{tabular}{|l|} 
Bin \\
Range \\
$(\mu \mathrm{m})$
\end{tabular} & \begin{tabular}{|l} 
Bin Mean \\
Diameter \\
$($ (um)
\end{tabular} & $\begin{array}{l}\text { Diff. of the } \\
\log \text { of the } \\
\text { Size }\end{array}$ & Counts & $\begin{array}{l}\text { Weighted } \\
\text { Moan } \\
\text { Diameter }\end{array}$ \\
\hline $0.30-0.49$ & .38 & 0.21 & 30400 & \multirow{6}{*}{1.34} \\
\hline $0.50-0.99$ & .70 & 0.30 & 69019 & \\
\hline $0.00-1.99$ & 1.41 & 0.30 & 22149 & \\
\hline $2.00-4.99$ & 3.16 & 0.40 & 24617 & \\
\hline $5.00-9.99$ & 7.07 & 0.30 & 1930 & \\
\hline$>10.00$ & 14.14 & 0.30 & 1255 & \\
\hline
\end{tabular}

\begin{tabular}{|c|c|c|c|c|}
\hline \multicolumn{5}{|c|}{ Sample \#10 } \\
\hline $\begin{array}{l}\text { Bin } \\
\text { Range } \\
(\mu \mathrm{m})\end{array}$ & \begin{tabular}{|l} 
Bin Mean \\
Diameter \\
$(u m)$
\end{tabular} & $\begin{array}{l}\text { Diff. of the } \\
\text { log of the } \\
\text { Size }\end{array}$ & Counts & $\begin{array}{l}\text { Weighted } \\
\text { Mean } \\
\text { Diameter }\end{array}$ \\
\hline $0.30-0.49$ & .38 & 0.21 & 33727 & \multirow{6}{*}{1.26} \\
\hline $0.50-0.99$ & .70 & 0.30 & 67665 & \\
\hline $1.00-1.99$ & 1.41 & 0.30 & 19620 & \\
\hline $2.00-4.99$ & 3.16 & 0.40 & 21511 & \\
\hline $5.00-9.99$ & 7.07 & 0.30 & 1685 & \\
\hline$>10.00$ & 14.14 & 0.30 & 1072 & \\
\hline
\end{tabular}




Sample \#11
\begin{tabular}{||c|c|c|r|r||}
\hline $\begin{array}{l}\text { Bin } \\
\text { Range } \\
\text { (um) }\end{array}$ & $\begin{array}{l}\text { Bin Mean } \\
\text { Diameter } \\
(\mathrm{mm})\end{array}$ & $\begin{array}{l}\text { Diff. of the } \\
\text { log of the } \\
\text { Size }\end{array}$ & Counts & $\begin{array}{l}\text { Weighted } \\
\text { Mean } \\
\text { Diameter }\end{array}$ \\
\hline $0.30-0.49$ & 0.38 & 0.21 & 29623 & \\
\hline $0.50-0.99$ & 0.70 & 0.30 & 70117 & \multirow{2}{*}{1.34} \\
\hline $1.00-1.99$ & 1.41 & 0.30 & 22715 & \\
\hline $2.00-4.99$ & 3.16 & 0.40 & 25541 & \\
\hline $5.00-9.99$ & 7.07 & 0.30 & 1903 & \\
\hline$>10.00$ & 14.14 & 0.30 & 1097 & \\
\hline
\end{tabular}

\begin{tabular}{|c|c|c|c|c|}
\hline $\begin{array}{l}\text { Bin } \\
\text { Range } \\
\text { (um) }\end{array}$ & \begin{tabular}{|l|} 
Bin Mean \\
Diameter \\
$(\omega \mathrm{m})$
\end{tabular} & $\begin{array}{l}\text { Diff. of the } \\
\log \text { of the } \\
\text { Size }\end{array}$ & Counts & $\begin{array}{l}\text { Weighted } \\
\text { Mean } \\
\text { Diameter }\end{array}$ \\
\hline $0.30-0.49$ & 0.38 & 0.21 & 35337 & \multirow{6}{*}{1.17} \\
\hline $0.50 \cdot 0.99$ & 0.70 & 0.30 & 62019 & \\
\hline $1.00-1.99$ & 1.41 & 0.30 & 17231 & \\
\hline $2.00-4.99$ & 3.16 & 0.40 & 18468 & \\
\hline $5.00-9.99$ & 7.07 & 0.30 & 1313 & \\
\hline$>10.00$ & 14.14 & 0.30 & 659 & \\
\hline
\end{tabular}

Sample \#12

\begin{tabular}{|c|c|c|c|c|}
\hline $\begin{array}{l}\text { Bin } \\
\text { Range } \\
(4 \mathrm{~m})\end{array}$ & $\begin{array}{l}\text { Bin Mean } \\
\text { Diameter } \\
(\mu \mathrm{m})\end{array}$ & $\begin{array}{l}\text { Diff. of the } \\
\text { log of the } \\
\text { Size }\end{array}$ & Counts & $\begin{array}{l}\text { Weighted } \\
\text { Mean } \\
\text { Diameter }\end{array}$ \\
\hline $0.30-0.49$ & .38 & 0.21 & 31202 & \multirow{6}{*}{1.29} \\
\hline $0.50-0.99$ & .70 & 0.30 & 67264 & \\
\hline $1.00-1.99$ & 1.41 & 0.30 & 21325 & \\
\hline $2.00-4.99$ & 3.16 & 0.40 & 23455 & \\
\hline $5.00-9.99$ & 7.07 & 0.30 & 1791 & \\
\hline$>10.00$ & 14.14 & 0.30 & 891 & \\
\hline
\end{tabular}

\begin{tabular}{|c|c|c|c|c|}
\hline \multicolumn{5}{|c|}{ Sample \#14 } \\
\hline $\begin{array}{l}\text { Bin } \\
\text { Range } \\
\text { (um) }\end{array}$ & \begin{tabular}{|l} 
Bin Mean \\
Diameter \\
$($ (um)
\end{tabular} & $\begin{array}{l}\text { Diff. of the } \\
\text { log of the } \\
\text { Size }\end{array}$ & Counts & $\begin{array}{l}\text { Weighted } \\
\text { Mean } \\
\text { Diameter }\end{array}$ \\
\hline $0.30-0.49$ & .38 & 0.21 & 33863 & \multirow{6}{*}{1.19} \\
\hline $0.50-0.99$ & .70 & 0.30 & 62928 & \\
\hline $1.00-1.99$ & 1.41 & 0.30 & 18073 & \\
\hline $2.00-4.99$ & 3.16 & 0.40 & 19529 & \\
\hline $5.00-9.99$ & 7.07 & 0.30 & 1317 & \\
\hline$>10.00$ & 14.14 & 0.30 & 675 & \\
\hline
\end{tabular}




\section{Aerosol Particle Measurement Record}

Description of sample location 3706 A Building, Resuspension Test, at $25 \mathrm{~cm}$, Date

8/11/94, Time sample started 9:03:59, Temperature 78.4 F, Humidity 40.1\%, Dew Point $51.9 \mathrm{~F}$, Average Wind Velocity (if applicable) $.01 \mathrm{~m} / \mathrm{s}$, \# of physical barriers between sample area and outside (if applicable) 1

\begin{tabular}{|c|c|c|c|c|}
\hline $\begin{array}{l}\text { Bin } \\
\text { Range } \\
(u m)\end{array}$ & \begin{tabular}{|l|} 
Bin Mean \\
Diameter \\
$(\mathrm{em})$
\end{tabular} & $\begin{array}{l}\text { Diff. of the } \\
\text { log of the } \\
\text { Size }\end{array}$ & Counts & $\begin{array}{l}\text { Weighted } \\
\text { Mean } \\
\text { Diameter }\end{array}$ \\
\hline $0.30-0.49$ & 0.38 & 0.21 & 64700 & \multirow{6}{*}{0.81} \\
\hline $0.50-0.99$ & 0.70 & 0.30 & 28784 & \\
\hline $1.00-1.99$ & 1.41 & 0.30 & 8132 & \\
\hline $2.00-4.99$ & 3.16 & 0.40 & 8239 & \\
\hline $5.00-9.99$ & 7.07 & 0.30 & 699 & \\
\hline$>10.00$ & 14.14 & 0.30 & 178 & \\
\hline \multicolumn{5}{|l|}{ Sample \#3 } \\
\hline $\begin{array}{l}\text { Bin } \\
\text { Range } \\
(u m)\end{array}$ & \begin{tabular}{|l|} 
Bin Mean \\
Diameter \\
$(\mu \mathrm{m})$
\end{tabular} & $\begin{array}{l}\text { Diff. of the } \\
\log \text { of the } \\
\text { Size }\end{array}$ & Counts & $\begin{array}{l}\text { Weighted } \\
\text { Mean } \\
\text { Diameter }\end{array}$ \\
\hline $0.30-0.49$ & 0.38 & 0.21 & 54973 & \multirow{6}{*}{1.13} \\
\hline $0.50-0.99$ & 0.70 & 0.30 & 26778 & \\
\hline $1.00-1.99$ & 1.41 & 0.30 & 8754 & \\
\hline $2.00-4.99$ & 3.16 & 0.40 & 10989 & \\
\hline $5.00-9.99$ & 7.07 & 0.30 & 1752 & \\
\hline$>10.00$ & 14.14 & 0.30 & 1326 & \\
\hline \multicolumn{5}{|l|}{ Sample \#5 } \\
\hline \begin{tabular}{|l} 
Bin \\
Range \\
(um)
\end{tabular} & \begin{tabular}{|l|} 
Bin Mean \\
Diameter \\
$(\mu / m)$
\end{tabular} & \begin{tabular}{|l|} 
Diff. of the \\
log of the \\
Size
\end{tabular} & Counts & \begin{tabular}{|l|} 
Weighted \\
Mean \\
Diameter
\end{tabular} \\
\hline $0.30-0.49$ & 0.38 & 0.21 & 60074 & \multirow{6}{*}{0.95} \\
\hline $0.50-0.99$ & 0.70 & 0.30 & 28340 & \\
\hline $1.00-1.99$ & 1.41 & 0.30 & 8313 & \\
\hline $2.00-4.99$ & 3.16 & 0.40 & 9363 & \\
\hline $5.00-9.99$ & 7.07 & 0.30 & 1151 & \\
\hline$>10.00$ & 14.14 & 0.30 & 724 & \\
\hline \multicolumn{5}{|l|}{ Sample \#7 } \\
\hline \begin{tabular}{|l|} 
Bin \\
Range \\
$(\mu \mathrm{m})$ \\
\end{tabular} & \begin{tabular}{|l|}
$\begin{array}{l}\text { Bin Mean } \\
\text { Diameter } \\
(\mu \mathrm{m})\end{array}$ \\
\end{tabular} & \begin{tabular}{|l|} 
Diff. of the \\
log of the \\
Size
\end{tabular} & Counts & \begin{tabular}{|l|} 
Weighted \\
Mean \\
Diameter \\
\end{tabular} \\
\hline $0.30 \cdot 0.49$ & 0.38 & 0.21 & 60432 & \multirow{6}{*}{0.93} \\
\hline $0.50-0.99$ & 0.70 & 0.30 & 28459 & \\
\hline $1.00-1.99$ & 1.41 & 0.30 & 8663 & \\
\hline $2.00-4.99$ & 3.16 & 0.40 & 9706 & \\
\hline $5.00-9.99$ & 7.07 & 0.30 & 1028 & \\
\hline$>10.00$ & 14.14 & 0.30 & 564 & \\
\hline \multicolumn{5}{|l|}{ Sample \#9 } \\
\hline $\begin{array}{l}\text { Bin } \\
\text { Range } \\
(\mu \mathrm{m})\end{array}$ & \begin{tabular}{|l|} 
Bin Mean \\
Diameter \\
$(\mu \mathrm{m})$
\end{tabular} & \begin{tabular}{|l|} 
Diff. of the \\
log of the \\
Size
\end{tabular} & Counts & $\begin{array}{l}\text { Weighted } \\
\text { Mean } \\
\text { Diameter }\end{array}$ \\
\hline $0.30-0.49$ & 0.38 & 0.21 & 58306 & \multirow{6}{*}{0.96} \\
\hline $0.50 \cdot 0.99$ & 0.70 & 0.30 & 29069 & \\
\hline $1.00-1.99$ & 1.41 & 0.30 & 9482 & \\
\hline $2.00-4.99$ & 3.16 & 0.40 & 10720 & \\
\hline $5.00-9.99$ & 7.07 & 0.30 & 1127 & \\
\hline$>10.00$ & 14.14 & 0.30 & 505 & \\
\hline
\end{tabular}

\begin{tabular}{l} 
Sample \#2 \\
\begin{tabular}{||c|c|c|r|r||}
\hline $\begin{array}{l}\text { Bin } \\
\text { Range } \\
(\mu \mathrm{m})\end{array}$ & $\begin{array}{l}\text { Bin Mean } \\
\text { Diameter } \\
(\mu \mathrm{m})\end{array}$ & $\begin{array}{l}\text { Diff. of the } \\
\text { log of the } \\
\text { Size }\end{array}$ & Counts & $\begin{array}{l}\text { Weighted } \\
\text { Mean } \\
\text { Diameter }\end{array}$ \\
\hline $0.30-0.49$ & .38 & 0.21 & 16399 & \\
\hline $0.50-0.99$ & .70 & 0.30 & 15845 \\
\cline { 1 - 4 } \\
\hline $1.00-1.99$ & 1.41 & 0.30 & 12842 \\
\hline $2.00-4.99$ & 3.16 & 0.40 & 50877 \\
\hline $5.00-9.99$ & 7.07 & 0.30 & 25062 \\
\hline$>10.00$ & 14.14 & 0.30 & 97629 \\
\hline
\end{tabular} \\
\hline
\end{tabular}

\begin{tabular}{|c|c|c|c|c|}
\hline \multicolumn{5}{|c|}{ Sample $\# 4$} \\
\hline $\begin{array}{l}\text { Bin } \\
\text { Range } \\
(\mu \mathrm{m})\end{array}$ & $\begin{array}{l}\text { Bin Mean } \\
\text { Diameter } \\
(\mu \mathrm{m})\end{array}$ & $\begin{array}{l}\text { Diff, of the } \\
\log \text { of the } \\
\text { Size }\end{array}$ & Counts & $\begin{array}{l}\text { Weighted } \\
\text { Mean } \\
\text { Diameter }\end{array}$ \\
\hline $0.30-0.49$ & .38 & 0.21 & 60265 & \multirow{6}{*}{0.96} \\
\hline $0.50-0.99$ & .70 & 0.30 & 27808 & \\
\hline $1.00-1.99$ & 1.41 & 0.30 & 8280 & \\
\hline $2.00-4.99$ & $\overline{3.16}$ & 0.40 & 9539 & \\
\hline $5.00-9.99$ & 7.07 & 0.30 & 1238 & \\
\hline$>10.00$ & 14.14 & 0.30 & 721 & \\
\hline
\end{tabular}

\begin{tabular}{|c|c|c|c|c|}
\hline \begin{tabular}{|l|} 
Bin \\
Range \\
(um)
\end{tabular} & \begin{tabular}{|l|} 
Bin Mean \\
Diameter \\
(um)
\end{tabular} & $\begin{array}{l}\text { Diff. of the } \\
\log \text { of the } \\
\text { Size }\end{array}$ & Counts & $\begin{array}{l}\text { Weighted } \\
\text { Mean } \\
\text { Diameter }\end{array}$ \\
\hline $0.30-0.49$ & .38 & 0.21 & 62022 & \multirow{6}{*}{0.89} \\
\hline $0.50-0.99$ & .70 & 0.30 & 27823 & \\
\hline $1.00-1.99$ & 1.41 & 0.30 & 8137 & \\
\hline $2.00-4.99$ & 3.16 & 0.40 & 8603 & \\
\hline $5.00-9.99$ & 7.07 & 0.30 & 1030 & \\
\hline$>10.00$ & 14.14 & 0.30 & 535 & \\
\hline
\end{tabular}

\begin{tabular}{|c|c|c|c|c|}
\hline $\begin{array}{l}\text { Bin } \\
\text { Range } \\
\text { (um) }\end{array}$ & \begin{tabular}{|l|} 
Bin Mean \\
Diameter \\
(um)
\end{tabular} & $\begin{array}{l}\text { Diff. of the } \\
\text { log of the } \\
\text { Size }\end{array}$ & Counts & $\begin{array}{l}\text { Weighted } \\
\text { Mean } \\
\text { Diameter }\end{array}$ \\
\hline $0.30-0.49$ & .38 & 0.21 & 60080 & \multirow{6}{*}{0.91} \\
\hline $0.50-0.99$ & .70 & 0.30 & 28884 & \\
\hline $0.00-1.99$ & 1.41 & 0.30 & $\overline{9082}$ & \\
\hline $2.00-4.99$ & $\overline{3.16}$ & 0.40 & 9848 & \\
\hline $5.00-9.99$ & 7.07 & 0.30 & 1011 & \\
\hline$>10.00$ & 14.14 & 0.30 & 400 & \\
\hline
\end{tabular}

\begin{tabular}{|c|c|c|c|c|}
\hline \multicolumn{5}{|c|}{ Sample \#10 } \\
\hline $\begin{array}{l}\text { Bin } \\
\text { Range } \\
\text { (um) }\end{array}$ & $\begin{array}{l}\text { Bin Mean } \\
\text { Diameter } \\
(\mu \mathrm{m})\end{array}$ & $\begin{array}{l}\text { Diff. of the } \\
\log \text { of the } \\
\text { Size }\end{array}$ & Counts & $\begin{array}{l}\text { Weighted } \\
\text { Mean } \\
\text { Diameter }\end{array}$ \\
\hline $0.30-0.49$ & .38 & 0.21 & 58299 & \multirow{6}{*}{0.95} \\
\hline $0.50-0.99$ & .70 & 0.30 & 28767 & \\
\hline $1.00-1.99$ & 1.41 & 0.30 & 9371 & \\
\hline $2.00-4.99$ & 3.16 & 0.40 & 10872 & \\
\hline $5.00-9.99$ & 7.07 & 0.30 & 1156 & \\
\hline$>10.00$ & 14.14 & 0.30 & 410 & \\
\hline
\end{tabular}


Sample \#11

\begin{tabular}{||l|l|l|r|r||}
\hline $\begin{array}{l}\text { Bin } \\
\text { Range } \\
(u \mathrm{~m})\end{array}$ & $\begin{array}{l}\text { Sin Mean } \\
\text { Diameter } \\
(\mu \mathrm{m})\end{array}$ & $\begin{array}{l}\text { Diff. of the } \\
\text { log of the } \\
\text { Size }\end{array}$ & Counts & $\begin{array}{l}\text { Weighted } \\
\text { Mean } \\
\text { Diameter }\end{array}$ \\
\hline $0.30-0.49$ & 0.38 & 0.21 & 48195 & \\
\hline $0.50 \cdot 0.99$ & 0.70 & 0.30 & 30037 \\
\hline $1.00-1.99$ & 1.41 & 0.30 & 12895 & \multirow{2}{*}{1.24} \\
\hline $2.00 \cdot 4.99$ & 3.16 & 0.40 & 17619 & \\
\hline $5.00-9.99$ & 7.07 & 0.30 & 2096 \\
\cline { 1 - 4 } & 10.00 & 14.14 & 0.30 & 705 \\
\hline
\end{tabular}

Sample \#13

\begin{tabular}{||l|l|l|r|r||}
\hline $\begin{array}{l}\text { Bin } \\
\text { Range } \\
(\mu \mathrm{m})\end{array}$ & $\begin{array}{l}\text { Bin Mean } \\
\text { Diameter } \\
(\mu \mathrm{m})\end{array}$ & $\begin{array}{l}\text { Diff. of the } \\
\text { log of the } \\
\text { Size }\end{array}$ & Counts & $\begin{array}{l}\text { Weighted } \\
\text { Mean } \\
\text { Diameter }\end{array}$ \\
\hline $0.30-0.49$ & 0.38 & 0.21 & 38658 & \\
\hline $0.50 \cdot 0.99$ & 0.70 & 0.30 & 31960 & \multirow{2}{*}{1.54} \\
\hline $1.00 \cdot 1.99$ & 1.41 & 0.30 & 16535 & \\
\hline $2.00-4.99$ & 3.16 & 0.40 & -26556 & \\
\hline $5.00-9.99$ & -7.07 & 0.30 & 3418 & \\
\hline$>10.00$ & 14.14 & 0.30 & 916 & \\
\hline
\end{tabular}

Sample \#12

\begin{tabular}{|c|c|c|c|c|}
\hline $\begin{array}{l}\text { Bin } \\
\text { Range } \\
\text { (um) }\end{array}$ & \begin{tabular}{|l|} 
Bin Mean \\
Diameter \\
$(\mu \mathrm{m})$
\end{tabular} & $\begin{array}{l}\text { Diff. of the } \\
\log \text { of the } \\
\text { Size }\end{array}$ & Counts & $\begin{array}{l}\text { Weighted } \\
\text { Mean } \\
\text { Diameter }\end{array}$ \\
\hline $0.30-0.49$ & .38 & 0.21 & 43136 & \multirow{6}{*}{1.38} \\
\hline $0.50-0.99$ & .70 & 0.30 & 31233 & \\
\hline $1.00-1.99$ & 1.41 & 0.30 & 14632 & \\
\hline $2.00-4.99$ & 3.16 & 0.40 & 21849 & \\
\hline $5.00-9.99$ & 7.07 & 0.30 & 2705 & \\
\hline$>10.00$ & 14.14 & 0.30 & 728 & \\
\hline
\end{tabular}

\begin{tabular}{|c|c|c|c|c|}
\hline $\begin{array}{l}\text { Bin } \\
\text { Range } \\
(\mathrm{mm})\end{array}$ & $\begin{array}{l}\text { Bin Mean } \\
\text { Diameter } \\
(\text { (um) }\end{array}$ & $\begin{array}{l}\text { Diff. of the } \\
\text { log of the } \\
\text { Size }\end{array}$ & Counts & $\begin{array}{l}\text { Weighted } \\
\text { Mean } \\
\text { Diameter }\end{array}$ \\
\hline $0.30-0.49$ & .38 & 0.21 & 38230 & \multirow{6}{*}{1.56} \\
\hline $0.50-0.99$ & .70 & 0.30 & 32113 & \\
\hline $1.00-1.99$ & 1.41 & 0.30 & 16597 & \\
\hline $2.00-4.99$ & 3.16 & 0.40 & 27089 & \\
\hline $5.00-9.99$ & 7.07 & 0.30 & 3550 & \\
\hline$>10.00$ & 14.14 & 0.30 & 953 & \\
\hline
\end{tabular}




\section{Aerosol Particle Measurement Record}

Description of sample location 3706A Building, Resuspension Test, at $1.5 \mathrm{~m}$, Date 8/11/94, Time sample started 10:00:14, Temperature78.8 F, Humidity 40.1\%, Dew Point $52.9 \mathrm{~F}$, Average Wind Velocity (if applicable) $.00 \mathrm{~m} / \mathrm{s}$, \# of physical barriers between sample area and outside (if applicable) 1

Sample \#1

\begin{tabular}{|c|c|c|c|c|}
\hline $\begin{array}{l}\text { Bin } \\
\text { Range } \\
(\mu \mathrm{m})\end{array}$ & $\begin{array}{l}\text { Bin Mean } \\
\text { Diameter } \\
(u m)\end{array}$ & $\begin{array}{l}\text { Diff. of the } \\
\text { log of the } \\
\text { Size }\end{array}$ & Counts & $\begin{array}{l}\text { Weighted } \\
\text { Mean } \\
\text { Diameter }\end{array}$ \\
\hline $0.30-0.49$ & 0.38 & 0.21 & 53095 & \multirow{6}{*}{0.78} \\
\hline $0.50-0.99$ & 0.70 & 0.30 & 50375 & \\
\hline $1.00-1.99$ & 1.41 & 0.30 & 7885 & \\
\hline $2.00-4.99$ & 3.16 & 0.40 & 7104 & \\
\hline $5.00-9.99$ & 7.07 & 0.30 & 312 & \\
\hline$>10.00$ & 14.14 & 0.30 & 123 & \\
\hline \multicolumn{5}{|l|}{ Sample \#3 } \\
\hline $\begin{array}{l}\text { Bin } \\
\text { Range } \\
\text { (um) }\end{array}$ & $\begin{array}{l}\text { Bin Mean } \\
\text { Diameter } \\
(\mu \mathrm{m})\end{array}$ & $\begin{array}{l}\text { Diff. of the } \\
\text { log of the } \\
\text { Size }\end{array}$ & Counts & $\begin{array}{l}\text { Weighted } \\
\text { Mean } \\
\text { Diameter }\end{array}$ \\
\hline $0.30-0.49$ & 0.38 & 0.21 & 48385 & \multirow{6}{*}{0.83} \\
\hline $0.50-0.99$ & 0.70 & 0.30 & 48669 & \\
\hline $1.00-1.99$ & 1.41 & 0.30 & 8572 & \\
\hline $2.00-4.99$ & 3.16 & 0.40 & 7744 & \\
\hline $5.00-9.99$ & 7.07 & 0.30 & 442 & \\
\hline$>10.00$ & 14.14 & 0.30 & 189 & \\
\hline \multicolumn{5}{|l|}{ Sample \#5 } \\
\hline $\begin{array}{l}\text { Bin } \\
\text { Range } \\
(\mu \mathrm{m}) \\
\end{array}$ & $\begin{array}{l}\text { Bin Mean } \\
\text { Diameter } \\
(\mu \mathrm{m})\end{array}$ & $\begin{array}{l}\text { Diff. of the } \\
\text { log of the } \\
\text { Size }\end{array}$ & Counts & $\begin{array}{l}\text { Weighted } \\
\text { Mean } \\
\text { Diameter }\end{array}$ \\
\hline $0.30-0.49$ & 0.38 & 0.21 & 20103 & \multirow{6}{*}{1.91} \\
\hline $0.50-0.99$ & 0.70 & 0.30 & 78718 & \\
\hline $1.00-1.99$ & 1.41 & 0.30 & 35768 & \\
\hline $2.00-4.99$ & 3.16 & 0.40 & 47436 & \\
\hline $5.00-9.99$ & 7.07 & 0.30 & 4870 & \\
\hline$>10.00$ & 14.14 & 0.30 & 4847 & \\
\hline \multicolumn{5}{|l|}{ Sample \#7 } \\
\hline $\begin{array}{l}\text { Bin } \\
\text { Range } \\
\text { (um) }\end{array}$ & $\begin{array}{l}\text { Bin Mean } \\
\text { Diameter } \\
(\mu \mathrm{m}) \\
\end{array}$ & $\begin{array}{l}\text { Diff. of the } \\
\text { log of the } \\
\text { Size }\end{array}$ & Counts & $\begin{array}{l}\text { Weighted } \\
\text { Mean } \\
\text { Diameter }\end{array}$ \\
\hline $0.30 \cdot 0.49$ & 0.38 & 0.21 & 20246 & \multirow{6}{*}{1.98} \\
\hline $0.50-0.99$ & 0.70 & 0.30 & 76640 & \\
\hline $1.00-1.99$ & 1.41 & 0.30 & 32255 & \\
\hline $2.00-4.99$ & 3.16 & 0.40 & 43478 & \\
\hline $5.00-9.99$ & 7.07 & 0.30 & 5196 & \\
\hline$>10.00$ & 14.14 & 0.30 & 5903 & \\
\hline
\end{tabular}

\begin{tabular}{|l|l|l|r|r||}
\hline \begin{tabular}{|l|l|r||} 
Sample \#9 \\
Range \\
$(\mathrm{mm})$
\end{tabular} & $\begin{array}{l}\text { Bin Mean } \\
\text { Diameter } \\
(\mathrm{mm})\end{array}$ & $\begin{array}{l}\text { Diff. of the } \\
\text { log of the } \\
\text { Size }\end{array}$ & $\begin{array}{l}\text { Counts } \\
\text { Meanhted } \\
\text { Diameter }\end{array}$ \\
\hline $0.30-0.49$ & 0.38 & 0.21 & 24370 & \\
\hline $0.50-0.99$ & 0.70 & 0.30 & 76054 & \multirow{2}{*}{1.65} \\
\hline $1.00-1.99$ & 1.41 & 0.30 & 29278 & \\
\hline $2.00-4.99$ & 3.16 & 0.40 & 35480 & \\
\hline $5.00-9.99$ & 7.07 & 0.30 & 3401 & \\
\hline$>10.00$ & 14.14 & 0.30 & 3098 & \\
\hline
\end{tabular}

Sample \#2

\begin{tabular}{||c|c|c|r|r||}
\hline $\begin{array}{l}\text { Bin } \\
\text { Range } \\
(\mu \mathrm{m})\end{array}$ & $\begin{array}{l}\text { Bin Mean } \\
\text { Diameter } \\
(\mathrm{mm})\end{array}$ & $\begin{array}{l}\text { Diff. of the } \\
\text { log of the } \\
\text { Size }\end{array}$ & Counts & $\begin{array}{l}\text { Weighted } \\
\text { Mean } \\
\text { Diameter }\end{array}$ \\
\hline $0.30-0.49$ & .38 & 0.21 & 49174 & \\
\cline { 1 - 4 } $0.50-0.99$ & .70 & 0.30 & 49849 & \multirow{2}{*}{0.85} \\
\hline $1.00-1.99$ & 1.41 & 0.30 & 8583 & \\
\hline $2.00-4.99$ & 3.16 & 0.40 & 8171 & \\
\hline $5.00-9.99$ & 7.07 & 0.30 & 469 & \\
\hline$>10.00$ & 14.14 & 0.30 & 287 & \\
\hline
\end{tabular}

Sample \#4

\begin{tabular}{||c|c|c|r|r|}
\hline $\begin{array}{l}\text { Bin } \\
(\mu \mathrm{m})\end{array}$ & $\begin{array}{l}\text { Bin Mean } \\
\text { Diameter } \\
(\mu \mathrm{m})\end{array}$ & $\begin{array}{l}\text { Diff. of the } \\
\text { log of the } \\
\text { Size }\end{array}$ & Counts & $\begin{array}{l}\text { Weighted } \\
\text { Mean } \\
\text { Diameter }\end{array}$ \\
\hline $0.30-0.49$ & .38 & 0.21 & 22559 & \\
\hline $0.50-0.99$ & .70 & 0.30 & 79257 & \multirow{2}{*}{1.68} \\
\hline $1.00-1.99$ & 1.41 & 0.30 & 33247 & \\
\hline $2.00-4.99$ & 3.16 & 0.40 & 42446 & \\
\hline $5.00-9.99$ & 7.07 & 0.30 & 3581 \\
\cline { 1 - 4 } \\
\hline$>10.00$ & 14.14 & 0.30 & 2785 & \\
\hline
\end{tabular}

Sample \#6

\begin{tabular}{|c|c|c|c|c|}
\hline $\begin{array}{l}\text { Bin } \\
\text { Range } \\
(\mu \mathrm{m})\end{array}$ & $\begin{array}{l}\text { Bin Mean } \\
\text { Diameter } \\
\text { (em) }\end{array}$ & $\begin{array}{l}\text { Diff. of the } \\
\text { log of the } \\
\text { Size }\end{array}$ & Counts & $\begin{array}{l}\text { Weighted } \\
\text { Mean } \\
\text { Diameter }\end{array}$ \\
\hline $0.30-0.49$ & .38 & 0.21 & 17597 & \\
\hline $0.50-0.99$ & .70 & 0.30 & 75611 & \\
\hline $1.00-1.99$ & 1.41 & 0.30 & 35956 & \\
\hline $2.00-4.99$ & 3.16 & 0.40 & 50855 & \\
\hline $5.00-9.99$ & 7.07 & 0.30 & 6296 & \\
\hline$>10.00$ & 14.14 & 0.30 & 7569 & \\
\hline
\end{tabular}

Sample \#8

\begin{tabular}{||c|c|c|r|r|}
\hline $\begin{array}{l}\text { Bin } \\
\text { Range } \\
(\mathrm{mm})\end{array}$ & $\begin{array}{l}\text { Bin Mean } \\
\text { Diameter } \\
(\mathrm{mm})\end{array}$ & $\begin{array}{l}\text { Diff. of the } \\
\text { log of the } \\
\text { Size }\end{array}$ & Counts & $\begin{array}{l}\text { Weighted } \\
\text { Mean } \\
\text { Diameter }\end{array}$ \\
\hline $0.30-0.49$ & .38 & 0.21 & 18514 & \\
\hline $0.50-0.99$ & .70 & 0.30 & 81849 & \multirow{2}{*}{2.02} \\
\hline $0.00-1.99$ & 1.41 & 0.30 & 40084 & \\
\hline $2.00-4.99$ & 3.16 & 0.40 & 54737 & \\
\hline $5.00-9.99$ & 7.07 & 0.30 & 5986 & \\
\hline$>10.00$ & 14.14 & 0.30 & 5799 & \\
\hline
\end{tabular}

\begin{tabular}{|c|c|c|c|c|}
\hline \multicolumn{5}{|c|}{ Sample \#10 } \\
\hline $\begin{array}{l}\text { Bin } \\
\text { Range } \\
(\mathrm{em})\end{array}$ & $\begin{array}{l}\text { Bin Mean } \\
\text { Diameter } \\
(\mathrm{u} / \mathrm{m})\end{array}$ & $\begin{array}{l}\text { Diff. of the } \\
\text { log of the } \\
\text { Size }\end{array}$ & Counts & $\begin{array}{l}\text { Weighted } \\
\text { Mean } \\
\text { Diameter }\end{array}$ \\
\hline $0.30-0.49$ & .38 & 0.21 & 21619 & \multirow{6}{*}{1.75} \\
\hline $0.50-0.99$ & .70 & 0.30 & 80628 & \\
\hline $1.00-1.99$ & 1.41 & 0.30 & 34301 & \\
\hline $2.00-4.99$ & 3.16 & 0.40 & 43920 & \\
\hline $5.00-9.99$ & 7.07 & 0.30 & 4001 & \\
\hline$>10.00$ & 14.14 & 0.30 & 3508 & \\
\hline
\end{tabular}


Sample \#11

\begin{tabular}{|c|c|c|c|c|}
\hline $\begin{array}{l}\text { Bin } \\
\text { Range } \\
(\mu \mathrm{m})\end{array}$ & $\begin{array}{l}\text { Bin Mean } \\
\text { Diameter } \\
(\mathrm{um})\end{array}$ & $\begin{array}{l}\text { Diff. of the } \\
\text { log of the } \\
\text { Size }\end{array}$ & Counts & $\begin{array}{l}\text { Weighted } \\
\text { Mean } \\
\text { Diameter }\end{array}$ \\
\hline $0.30-0.49$ & 0.38 & 0.21 & 21619 & \multirow{6}{*}{1.74} \\
\hline $0.50-0.99$ & 0.70 & 0.30 & 80628 & \\
\hline $1.00-1.99$ & 1.41 & 0.30 & 34301 & \\
\hline $2.00-4.99$ & 3.16 & 0.40 & 43920 & \\
\hline $5.00-9.99$ & 7.07 & 0.30 & 4001 & \\
\hline$>10.00$ & 14.14 & 0.30 & 3290 & \\
\hline
\end{tabular}

Sample \#13

\begin{tabular}{||c|c|c|r|r||}
\hline $\begin{array}{l}\text { Bin } \\
\text { Range } \\
(\mu \mathrm{m})\end{array}$ & $\begin{array}{l}\text { Bin Mean } \\
\text { Diameter } \\
(\mu \mathrm{m})\end{array}$ & $\begin{array}{l}\text { Diff. of the } \\
\text { log of the } \\
\text { Size }\end{array}$ & Counts & $\begin{array}{l}\text { Weighted } \\
\text { Mean } \\
\text { Diameter }\end{array}$ \\
\hline $0.30-0.49$ & 0.38 & 0.21 & 23024 & \\
\hline $0.50-0.99$ & 0.70 & 0.30 & 79035 & \multirow{2}{*}{1.62} \\
\hline $1.00-1.99$ & 1.41 & 0.30 & 33505 & \\
\hline $2.00-4.99$ & 3.16 & 0.40 & 41468 & \\
\hline $5.00-9.99$ & 7.07 & 0.30 & 3383 & \\
\hline$>10.00$ & 14.14 & 0.30 & 2011 & \\
\hline
\end{tabular}

$-$
Samole \#12

\begin{tabular}{||c|c|c|r|r||}
\hline $\begin{array}{l}\text { Bin } \\
\text { Range } \\
(\mu \mathrm{m})\end{array}$ & $\begin{array}{l}\text { Bin Mean } \\
\text { Diameter } \\
(\mathrm{\mu m})\end{array}$ & $\begin{array}{l}\text { Diff. of the } \\
\text { log of the } \\
\text { Size }\end{array}$ & $\begin{array}{l}\text { Counts } \\
\text { Meighted } \\
\text { Diameter }\end{array}$ \\
\hline $0.30-0.49$ & .38 & 0.21 & 22077 & \\
\hline $0.50-0.99$ & .70 & 0.30 & 79158 & \multirow{2}{*}{1.71} \\
\hline $1.00-1.99$ & 1.41 & 0.30 & 34222 & \\
\hline $2.00-4.99$ & 3.16 & 0.40 & 43605 & \\
\hline $5.00-9.99$ & 7.07 & 0.30 & 3896 & \\
\hline$>10.00$ & 14.14 & 0.30 & 2837 & \\
\hline
\end{tabular}

Sample \#14

\begin{tabular}{||c|c|c|r|r|}
\hline $\begin{array}{l}\text { Bin } \\
(\mu \mathrm{m})\end{array}$ & $\begin{array}{l}\text { Bin Mean } \\
\text { Diameter } \\
(\mathrm{\mu m})\end{array}$ & $\begin{array}{l}\text { Diff. of the } \\
\text { log of the } \\
\text { Size }\end{array}$ & Counts & $\begin{array}{l}\text { Weighted } \\
\text { Mean } \\
\text { Diameter }\end{array}$ \\
\hline $0.30-0.49$ & .38 & 0.21 & 22581 & \\
\hline $0.50-0.99$ & .70 & 0.30 & 78088 & \multirow{2}{*}{1.66} \\
\hline $1.00-1.99$ & 1.41 & 0.30 & 33236 & \\
\hline $2.00-4.99$ & 3.16 & 0.40 & 41367 & \\
\hline $5.00-9.99$ & 7.07 & 0.30 & 3639 & \\
\hline$>90.00$ & 14.14 & 0.30 & 2367 & \\
\hline
\end{tabular}




\section{Aerosol Particle Measurement Record}

Description of sample location 3706A Building, Resuspension Test, at $25 \mathrm{~cm}$, Date 8/11/94, Time sample started 10:00:14, Temperature 78.8 F, Humidity 40.1\%, Dew Point $52.9 \mathrm{~F}$, Average Wind Velocity (if applicable) $.00 \mathrm{~m} / \mathrm{s}$, \# of physical barriers between sample area and outside (if applicable) 1

\begin{tabular}{|c|c|c|c|c|}
\hline $\begin{array}{l}\text { Bin } \\
\text { Range } \\
\text { (um) }\end{array}$ & \begin{tabular}{|l} 
Bin Mean \\
Diameter \\
(urm)
\end{tabular} & $\begin{array}{l}\text { Diff. of the } \\
\log \text { of the } \\
\text { Size }\end{array}$ & Counts & $\begin{array}{l}\text { Weighted } \\
\text { Mean } \\
\text { Diameter }\end{array}$ \\
\hline $0.30-0.49$ & 0.38 & 0.21 & 60148 & \multirow{6}{*}{0.89} \\
\hline $0.50-0.99$ & 0.70 & 0.30 & 28678 & \\
\hline $1.00-1.99$ & 1.41 & 0.30 & 9062 & \\
\hline $2.00-4.99$ & 3.16 & 0.40 & 10062 & \\
\hline $5.00-9.99$ & 7.07 & 0.30 & 909 & \\
\hline$>10.00$ & 14.14 & 0.30 & 257 & \\
\hline \multicolumn{5}{|l|}{ Sample \#3 } \\
\hline $\begin{array}{l}\text { Bin } \\
\text { Range } \\
(\mu \mathrm{m})\end{array}$ & $\begin{array}{l}\text { Bin Mean } \\
\text { Diameter } \\
(\mathrm{km})\end{array}$ & $\begin{array}{l}\text { Diff. of the } \\
\text { log of the } \\
\text { Size }\end{array}$ & Counts & $\begin{array}{l}\text { Weighted } \\
\text { Mean } \\
\text { Diameter }\end{array}$ \\
\hline $0.30-0.49$ & 0.38 & 0.21 & 29968 & \multirow{6}{*}{2.12} \\
\hline $0.50-0.99$ & 0.70 & 0.30 & 32905 & \\
\hline $1.00-1.99$ & 1.41 & 0.30 & 19600 & \\
\hline $2.00-4.99$ & 3.16 & 0.40 & 35828 & \\
\hline $5.00-9.99$ & 7.07 & 0.30 & 6217 & \\
\hline$>10.00$ & 14.14 & 0.30 & 3692 & \\
\hline \multicolumn{5}{|l|}{ Sample \#5 } \\
\hline \begin{tabular}{|l|} 
Bin \\
Range \\
$(\mathrm{mm})$
\end{tabular} & $\begin{array}{l}\text { Bin Mean } \\
\text { Diameter } \\
\text { (im) }\end{array}$ & \begin{tabular}{|l|} 
Diff. of the \\
log of the \\
Size
\end{tabular} & Counts & $\begin{array}{l}\text { Weighted } \\
\text { Mean } \\
\text { Diameter }\end{array}$ \\
\hline $0.30 \cdot 0.49$ & 0.38 & 0.21 & 37683 & \multirow{6}{*}{1.70} \\
\hline $0.50-0.99$ & 0.70 & 0.30 & 33109 & \\
\hline $1.00-1.99$ & 1.41 & 0.30 & 17086 & \\
\hline $2.00-4.99$ & 3.16 & 0.40 & 26132 & \\
\hline $5.00-9.99$ & 7.07 & 0.30 & 3931 & \\
\hline$>10.00$ & 14.14 & 0.30 & 2258 & \\
\hline \multicolumn{5}{|l|}{ Sample \#7 } \\
\hline $\begin{array}{l}\text { Bin } \\
\text { Range } \\
\text { (um) }\end{array}$ & \begin{tabular}{|l} 
Bin Mean \\
Diameter \\
$(\mathrm{um})$
\end{tabular} & $\begin{array}{l}\text { Diff. of the } \\
\text { log of the } \\
\text { Size }\end{array}$ & Counts & \begin{tabular}{|l} 
Weighted \\
Mean \\
Diameter
\end{tabular} \\
\hline $0.30-0.49$ & 0.38 & 0.21 & 44786 & \multirow{6}{*}{$\begin{array}{c}1.38 \\
.\end{array}$} \\
\hline $0.50 \cdot 0.99$ & 0.70 & 0.30 & 31099 & \\
\hline $1.00-1.99$ & 1.41 & 0.30 & 13877 & \\
\hline $2,00 \cdot 4.99$ & 3.16 & 0.40 & 19520 & \\
\hline $5.00-9.99$ & 7.07 & 0.30 & 2529 & \\
\hline$>10.00$ & 14.14 & 0.30 & 1327 & \\
\hline \multicolumn{5}{|l|}{ Sample \#9 } \\
\hline $\begin{array}{l}\text { Bin } \\
\text { Range } \\
(\mu \mathrm{m})\end{array}$ & \begin{tabular}{|l} 
Bin Mean \\
Diameter \\
$(u \mathrm{~m})$
\end{tabular} & $\begin{array}{l}\text { Diff. of the } \\
\text { log of the } \\
\text { Size }\end{array}$ & Counts & $\begin{array}{l}\text { Weighted } \\
\text { Mean } \\
\text { Diameter }\end{array}$ \\
\hline $0.30-0.49$ & 0.38 & 0.21 & 27216 & \multirow{6}{*}{2.17} \\
\hline $0.50-0.99$ & 0.70 & 0.30 & 37254 & \\
\hline $1.00-1.99$ & 1.41 & 0.30 & 28245 & \\
\hline $2.00-4.99$ & 3.16 & 0.40 & 54877 & \\
\hline $5.00-9.99$ & 7.07 & 0.30 & 8051 & \\
\hline$>10.00$ & 14.14 & 0.30 & 2633 & \\
\hline
\end{tabular}

Sample \#2

\begin{tabular}{|c|c|c|c|c|}
\hline $\begin{array}{l}\text { Bin } \\
\text { Range } \\
(\mu \mathrm{m})\end{array}$ & \begin{tabular}{|l} 
Bin Mean \\
Diameter \\
$(\mathrm{um})$
\end{tabular} & $\begin{array}{l}\text { Diff. of the } \\
\log \text { of the } \\
\text { Size }\end{array}$ & Counts & $\begin{array}{l}\text { Weighted } \\
\text { Mean } \\
\text { Diameter }\end{array}$ \\
\hline $0.30-0.49$ & .38 & 0.21 & 13166 & \\
\hline $0.50-0.99$ & .70 & 0.30 & 8245 & \\
\hline $1.00-1.99$ & 1.41 & 0.30 & 4116 & \\
\hline $2.00-4.99$ & 3.16 & 0.40 & 18794 & \\
\hline $5.00-9.99$ & 7.07 & 0.30 & 16652 & \\
\hline$>10.00$ & 14.14 & 0.30 & 179781 & \\
\hline \multicolumn{5}{|l|}{ Sample \#4 } \\
\hline $\begin{array}{l}\text { Bin } \\
\text { Range } \\
(\mu \mathrm{m}) \\
\end{array}$ & \begin{tabular}{|l|} 
Bin Mean \\
Diameter \\
$(u m)$
\end{tabular} & $\begin{array}{l}\text { Diff. of the } \\
\text { log of the } \\
\text { Size }\end{array}$ & Counts & $\begin{array}{l}\text { Weighted } \\
\text { Mean } \\
\text { Diameter }\end{array}$ \\
\hline $0.30-0.49$ & .38 & 0.21 & 23109 & \multirow{6}{*}{2.47} \\
\hline $0.50-0.99$ & .70 & 0.30 & 38133 & \\
\hline $1.00-1.99$ & 1.41 & 0.30 & 28181 & \\
\hline $2.00-4.99$ & 3.16 & 0.40 & 55870 & \\
\hline $5.00-9.99$ & 7.07 & 0.30 & 9419 & \\
\hline$>10.00$ & 14.14 & 0.30 & 5481 & \\
\hline
\end{tabular}
Sample $\# 6$

\begin{tabular}{|c|c|c|c|c|}
\hline \begin{tabular}{|l} 
Bin \\
Range \\
$(\mu \mathrm{m})$ \\
\end{tabular} & \begin{tabular}{|l} 
Bin Mean \\
Diameter \\
$(\mu \mathrm{m})$
\end{tabular} & $\begin{array}{l}\text { Diff. of the } \\
\log \text { of the } \\
\text { Size }\end{array}$ & Counts & $\begin{array}{l}\text { Woighted } \\
\text { Mean } \\
\text { Diamoter }\end{array}$ \\
\hline $0.30-0.49$ & .38 & 0.21 & 45919 & \multirow{6}{*}{1.38} \\
\hline $0.50-0.99$ & .70 & 0.30 & 31427 & \\
\hline $1.00-1.99$ & 1.41 & 0.30 & 13453 & \\
\hline $2.00-4.99$ & 3.16 & 0.40 & 18923 & \\
\hline $5.00-9.99$ & 7.07 & 0.30 & 2621 & \\
\hline$>10.00$ & 94.14 & 0.30 & 1433 & \\
\hline
\end{tabular}

\begin{tabular}{|c|c|c|c|c|}
\hline $\begin{array}{l}\text { Bin } \\
\text { Range } \\
(\omega \mathrm{m})\end{array}$ & $\begin{array}{l}\text { Bin Mean } \\
\text { Diameter } \\
(\text { (um) }\end{array}$ & $\begin{array}{l}\text { Diff. of the } \\
\text { log of the } \\
\text { Size }\end{array}$ & Counts & $\begin{array}{l}\text { Woighted } \\
\text { Mean } \\
\text { Diameter }\end{array}$ \\
\hline $0.30-0.49$ & .38 & 0.21 & 37071 & \multirow{6}{*}{1.67} \\
\hline $0.50-0.99$ & .70 & 0.30 & 33998 & \\
\hline $0.00-1.99$ & 1.41 & 0.30 & 18089 & \\
\hline $2.00-4.99$ & 3.16 & 0.40 & 29223 & \\
\hline $5.00-9.99$ & 7.07 & 0.30 & 3990 & \\
\hline$>10.00$ & 14.14 & 0.30 & 1595 & \\
\hline
\end{tabular}

\begin{tabular}{|c|c|c|c|c|}
\hline \multicolumn{5}{|c|}{ Sample \#10 } \\
\hline \begin{tabular}{|l} 
Bin \\
Range \\
$(\mu \mathrm{m})$
\end{tabular} & $\begin{array}{l}\text { Bin Mean } \\
\text { Diameter } \\
(u m)\end{array}$ & $\begin{array}{l}\text { Diff. of the } \\
\log \text { of the } \\
\text { Size }\end{array}$ & Counts & $\begin{array}{l}\text { Woighted } \\
\text { Moan } \\
\text { Diameter }\end{array}$ \\
\hline $0.30-0.49$ & .38 & 0.21 & 24015 & \multirow{6}{*}{2.20} \\
\hline $0.50-0.99$ & .70 & 0.30 & 38176 & \\
\hline $1.00-1.99$ & 1.41 & 0.30 & 28245 & \\
\hline $2.00-4.99$ & $\overline{3.16}$ & 0.40 & 54877 & \\
\hline $5.00-9.99$ & 7.07 & 0.30 & 8051 & \\
\hline$>10.00$ & 14.14 & 0.30 & 2633 & \\
\hline
\end{tabular}


Sample \#11

\begin{tabular}{|c|c|c|c|c|}
\hline $\begin{array}{l}\text { Bin } \\
\text { Range } \\
\text { (um) }\end{array}$ & $\begin{array}{l}\text { Bin Mean } \\
\text { Diameter } \\
(\mathrm{um})\end{array}$ & $\begin{array}{l}\text { Diff. of the } \\
\text { log of the } \\
\text { Size }\end{array}$ & Counts & $\begin{array}{l}\text { Weighted } \\
\text { Mean } \\
\text { Diameter }\end{array}$ \\
\hline $0.30-0.49$ & 0.38 & 0.21 & 25084 & \multirow{6}{*}{2.15} \\
\hline $0.50-0.99$ & 0.70 & 0.30 & 37704 & \\
\hline $1.00-1.99$ & 1.41 & 0.30 & 26883 & \\
\hline $2.00-4.99$ & 3.16 & 0.40 & 51270 & \\
\hline $5.00-9.99$ & 7.07 & 0.30 & 7654 & \\
\hline$>10.00$ & $\longdiv { 1 4 . 1 4 }$ & 0.30 & 2510 & \\
\hline
\end{tabular}

Sample \#13

\begin{tabular}{|c|c|c|c|c|}
\hline \begin{tabular}{|l} 
Bin \\
Range \\
$(\mu \mathrm{m})$
\end{tabular} & \begin{tabular}{|l|} 
Bin Mean \\
Diameter \\
$(u m)$
\end{tabular} & $\begin{array}{l}\text { Diff. of the } \\
\log \text { of the } \\
\text { Size }\end{array}$ & Counts & $\begin{array}{l}\text { Weighted } \\
\text { Mean } \\
\text { Diameter }\end{array}$ \\
\hline $0.30-0.49$ & 0.38 & 0.21 & 25407 & \multirow{6}{*}{2.11} \\
\hline $0.50 \cdot 0.99$ & 0.70 & 0.30 & 37250 & \\
\hline $1.00-1.99$ & 1.41 & 0.30 & 26526 & \\
\hline $2.00 \cdot 4.99$ & 3.16 & 0.40 & 50124 & \\
\hline $5.00-9.99$ & 7.07 & 0.30 & $\overline{7406}$ & \\
\hline$>10.00$ & 14.14 & 0.30 & 2172 & \\
\hline
\end{tabular}

$\therefore$
Samole \#12

\begin{tabular}{|c|c|c|c|c|}
\hline $\begin{array}{l}\text { Bin } \\
\text { Range } \\
(u m)\end{array}$ & \begin{tabular}{|l|} 
Bin Mean \\
Diameter \\
$(\mu \mathrm{m})$
\end{tabular} & $\begin{array}{l}\text { Diff. of the } \\
\text { log of the } \\
\text { Size }\end{array}$ & Counts & $\begin{array}{l}\text { Weighted } \\
\text { Mean } \\
\text { Diameter }\end{array}$ \\
\hline $0.30 \cdot 0.49$ & .38 & 0.21 & 24025 & \multirow{6}{*}{2.19} \\
\hline $0.50-0.99$ & .70 & 0.30 & 37727 & \\
\hline $1.00-1.99$ & 1.41 & 0.30 & 27806 & \\
\hline $2.00-4.99$ & 3.16 & 0.40 & 53583 & \\
\hline $5.00-9.99$ & 7.07 & 0.30 & 7991 & \\
\hline$>10.00$ & 14.14 & 0.30 & 2517 & \\
\hline
\end{tabular}

\begin{tabular}{|c|c|c|c|c|}
\hline $\begin{array}{l}\text { Bin } \\
\text { Range } \\
(\mathrm{um})\end{array}$ & \begin{tabular}{|l} 
Bin Mean \\
Diameter \\
$(\mathrm{km})$
\end{tabular} & $\begin{array}{l}\text { Diff. of the } \\
\text { log of the } \\
\text { Size }\end{array}$ & Counts & $\begin{array}{l}\text { Weighted } \\
\text { Mean } \\
\text { Diameter }\end{array}$ \\
\hline $0.30-0.49$ & .38 & 0.21 & 26207 & \multirow{6}{*}{2.05} \\
\hline $0.50-0.99$ & .70 & 0.30 & 36808 & \\
\hline $1.00-1.99$ & 1.41 & 0.30 & 25038 & \\
\hline $2.00-4.99$ & 3.16 & 0.40 & 47401 & \\
\hline $5.00-9.99$ & 7.07 & 0.30 & 6731 & \\
\hline$>10.00$ & 14.14 & 0.30 & 1915 & \\
\hline
\end{tabular}




\section{Aerosol Particle Measurement Record}

Description of sample location 3706A Building, Resuspension Test, at $1.5 \mathrm{~m}$, Date 8/12/94, Time sample started 7:32:55, Temperature80.4 F, Humidity 40.1\%, Dew Point $55.0 \mathrm{~F}$, Average Wind Velocity (if applicable) $.00 \mathrm{~m} / \mathrm{s}$, \# of physical barriers between sample area and outside (if applicable) 1

\begin{tabular}{|c|c|c|c|c|}
\hline $\begin{array}{l}\text { Bin } \\
\text { Range } \\
(u m)\end{array}$ & \begin{tabular}{|l|} 
Bin Mean \\
Diameter \\
(urm)
\end{tabular} & \begin{tabular}{|l|} 
Diff. of the \\
log of the \\
Size
\end{tabular} & Counts & $\begin{array}{l}\text { Weighted } \\
\text { Mean } \\
\text { Diameter }\end{array}$ \\
\hline $0.30-0.49$ & 0.38 & 0.21 & 60030 & \multirow{6}{*}{0.63} \\
\hline $0.50-0.99$ & 0.70 & 0.30 & 47283 & \\
\hline $1.00-1.99$ & 1.41 & 0.30 & 4571 & \\
\hline $2.00-4.99$ & 3.16 & 0.40 & 2667 & \\
\hline $5.00-9.99$ & 7.07 & 0.30 & 112 & \\
\hline$>10.00$ & 14.14 & 0.30 & 49 & \\
\hline \multicolumn{5}{|l|}{ Sample \#3 } \\
\hline $\begin{array}{l}\text { Bin } \\
\text { Range } \\
(\mu \mathrm{m})\end{array}$ & \begin{tabular}{|l|} 
Bin Mean \\
Diameter \\
(um)
\end{tabular} & \begin{tabular}{|l|} 
Diff. of the \\
log of the \\
Size
\end{tabular} & Counts & $\begin{array}{l}\text { Weighted } \\
\text { Mean } \\
\text { Diameter }\end{array}$ \\
\hline $0.30 \cdot 0.49$ & 0.38 & 0.21 & 60438 & \multirow{6}{*}{0.6} \\
\hline $0.50-0.99$ & 0.70 & 0.30 & 47589 & \\
\hline $1.00-1.99$ & 1.41 & 0.30 & 4696 & \\
\hline $2.00-4.99$ & 3.16 & 0.40 & 2816 & \\
\hline $5.00-9.99$ & 7.07 & 0.30 & 129 & \\
\hline$>10.00$ & 14.14 & 0.30 & 66 & \\
\hline \multicolumn{5}{|l|}{ Sample \#5 } \\
\hline \begin{tabular}{|l} 
Bin \\
Range \\
$(u m)$
\end{tabular} & $\begin{array}{l}\text { Bin Mean } \\
\text { Diameter } \\
\text { (um) }\end{array}$ & \begin{tabular}{|l|} 
Diff. of the \\
log of the \\
Size
\end{tabular} & Counts & $\begin{array}{l}\text { Weighted } \\
\text { Mean } \\
\text { Diameter }\end{array}$ \\
\hline $0.30-0.49$ & 0.38 & 0.21 & 46700 & \multirow{6}{*}{0.91} \\
\hline $0.50-0.99$ & 0.70 & 0.30 & 59193 & \\
\hline $1.00-1.99$ & 1.41 & 0.30 & 11780 & \\
\hline $2.00 \cdot 4.99$ & 3.16 & 0.40 & 10484 & \\
\hline $5.00-9.99$ & 7.07 & 0.30 & 570 & \\
\hline$>10.00$ & 14.14 & 0.30 & 331 & \\
\hline \multicolumn{5}{|l|}{ Sample $\# 7$} \\
\hline $\begin{array}{l}\text { Bin } \\
\text { Range } \\
(\mu \mathrm{m})\end{array}$ & \begin{tabular}{|l|} 
Bin Mean \\
Diameter \\
(um)
\end{tabular} & $\begin{array}{l}\text { Diff. of the } \\
\text { log of the } \\
\text { Size }\end{array}$ & Counts & $\begin{array}{l}\text { Weighted } \\
\text { Mean } \\
\text { Diameter }\end{array}$ \\
\hline $0.30-0.49$ & 0.38 & 0.21 & 23434 & \multirow{6}{*}{1.59} \\
\hline $0.50-0.99$ & 0.70 & 0.30 & 84943 & \\
\hline $1.00-1.99$ & 1.41 & 0.30 & 37997 & \\
\hline $2.00-4.99$ & 3.16 & 0.40 & 45683 & \\
\hline $5.00-9.99$ & 7.07 & 0.30 & 3108 & \\
\hline$>10.00$ & 14.14 & 0.30 & 1708 & \\
\hline \multicolumn{5}{|l|}{ Sample $\$ 9$} \\
\hline $\begin{array}{l}\text { Bin } \\
\text { Range } \\
(\mu \mathrm{m})\end{array}$ & \begin{tabular}{|l|} 
Bin Mean \\
Diameter \\
$(\mu \mathrm{m})$
\end{tabular} & \begin{tabular}{|l|} 
Diff. of the \\
log of the \\
Size
\end{tabular} & Counts & $\begin{array}{l}\text { Weighted } \\
\text { Mean } \\
\text { Diameter }\end{array}$ \\
\hline $0.30-0.49$ & 0.38 & 0.21 & 19975 & \\
\hline $0.50-0.99$ & 0.70 & 0.30 & 88796 & \\
\hline $1.00-1.99$ & 1.41 & 0.30 & 44793 & \\
\hline $2.00-4.99$ & 3.16 & 0.40 & 58894 & \\
\hline $5.00 \cdot 9.99$ & 7.07 & 0.30 & 4206 & \\
\hline$>10.00$ & 14.14 & 0.30 & 2121 & \\
\hline
\end{tabular}

\begin{tabular}{|c|c|c|c|c|}
\hline $\begin{array}{l}\text { Bin } \\
\text { Range } \\
\text { (um) }\end{array}$ & \begin{tabular}{|l|} 
Bin Mean \\
Diameter \\
$(\mathrm{em})$
\end{tabular} & $\begin{array}{l}\text { Diff. of the } \\
\log \text { of the } \\
\text { Size }\end{array}$ & Counts & $\begin{array}{l}\text { Weighted } \\
\text { Mean } \\
\text { Diameter }\end{array}$ \\
\hline $0.30-0.49$ & .38 & 0.21 & 63172 & \multirow{6}{*}{0.63} \\
\hline $0.50-0.99$ & .70 & 0.30 & 49890 & \\
\hline $1.00-1.99$ & 1.41 & 0.30 & 4535 & \\
\hline $2.00-4.99$ & 3.16 & 0.40 & 2748 & \\
\hline $5.00-9.99$ & 7.07 & 0.30 & 126 & \\
\hline$>10.00$ & 14.14 & 0.30 & 48 & \\
\hline \multicolumn{5}{|l|}{ Sample \#4 } \\
\hline \begin{tabular}{|l|} 
Bin \\
Range \\
(um)
\end{tabular} & $\begin{array}{l}\text { Bin Mean } \\
\text { Diameter } \\
(\mathrm{um})\end{array}$ & $\begin{array}{l}\text { Diff. of the } \\
\text { log of the } \\
\text { Size }\end{array}$ & Counts & $\begin{array}{l}\text { Weighted } \\
\text { Mean } \\
\text { Diameter }\end{array}$ \\
\hline $0.30-0.49$ & .38 & 0.21 & 59802 & \multirow{6}{*}{0.64} \\
\hline $0.50-0.99$ & .70 & 0.30 & 48647 & \\
\hline $1.00-1.99$ & 1.41 & 0.30 & 4770 & \\
\hline $2.00-4.99$ & 3.16 & 0.40 & 3028 & \\
\hline $5.00-9.99$ & 7.07 & 0.30 & 121 & \\
\hline$>10.00$ & 14.14 & 0.30 & 55 & \\
\hline
\end{tabular}

\begin{tabular}{|c|c|c|c|c|}
\hline \multicolumn{5}{|l|}{ Sample \#6 } \\
\hline $\begin{array}{l}\text { Bin } \\
\text { Range } \\
\text { (um) }\end{array}$ & \begin{tabular}{|l} 
Bin Mean \\
Diameter \\
$(\mathrm{lm})$
\end{tabular} & $\begin{array}{l}\text { Diff. of the } \\
\log \text { of the } \\
\text { Size }\end{array}$ & Counts & $\begin{array}{l}\text { Weighted } \\
\text { Mean } \\
\text { Diameter }\end{array}$ \\
\hline $0.30-0.49$ & .38 & 0.21 & 39808 & \multirow{6}{*}{1.06} \\
\hline $0.50-0.99$ & .70 & 0.30 & 63973 & \\
\hline $1.00-1.99$ & 1.41 & 0.30 & 15980 & \\
\hline $2.00-4.99$ & 3.16 & 0.40 & 15215 & \\
\hline $5.00-9.99$ & 7.07 & 0.30 & 948 & \\
\hline$>10.00$ & 14.94 & 0.30 & 570 & \\
\hline
\end{tabular}

\begin{tabular}{|c|c|c|c|c|}
\hline \\
\hline $\begin{array}{l}\text { Bin } \\
\text { Range } \\
\text { (um) }\end{array}$ & \begin{tabular}{|l|} 
Bin Mean \\
Diameter \\
$(\mu \mathrm{m})$
\end{tabular} & $\begin{array}{l}\text { Diff. of the } \\
\log \text { of the } \\
\text { Size }\end{array}$ & Counts & $\begin{array}{l}\text { Weighted } \\
\text { Mean } \\
\text { Diamoter }\end{array}$ \\
\hline $0.30-0.49$ & .38 & 0.21 & 21882 & \multirow{6}{*}{1.65} \\
\hline $0.50-0.99$ & .70 & 0.30 & 86541 & \\
\hline $0.00-1.99$ & 1.41 & 0.30 & 40620 & \\
\hline $2.00-4.99$ & 3.16 & 0.40 & 50950 & \\
\hline $5.00-9.99$ & 7.07 & 0.30 & 3589 & \\
\hline$>10.00$ & 14.14 & 0.30 & 1941 & \\
\hline
\end{tabular}

\begin{tabular}{|c|c|c|c|c|}
\hline \multicolumn{5}{|c|}{ Sample $\# 10$} \\
\hline $\begin{array}{l}\text { Bin } \\
\text { Range } \\
\text { (em)! }\end{array}$ & $\begin{array}{l}\text { Bin Mean } \\
\text { Diameter } \\
(\mu \mathrm{m})\end{array}$ & $\begin{array}{l}\text { Diff. of the } \\
\log \text { of the } \\
\text { Size }\end{array}$ & Counts & $\begin{array}{l}\text { Weighted } \\
\text { Mean } \\
\text { Diameter }\end{array}$ \\
\hline $0.30-0.49$ & .38 & 0.21 & 20976 & \multirow{6}{*}{1.66} \\
\hline $0.50-0.99$ & .70 & 0.30 & 87440 & \\
\hline $1.00-1.99$ & 1.41 & 0.30 & 41972 & \\
\hline $2.00-4.99$ & 3.16 & 0.40 & 52431 & \\
\hline $5.00-9.99$ & 7.07 & 0.30 & 3702 & \\
\hline$>10.00$ & 14.14 & 0.30 & 1805 & \\
\hline
\end{tabular}


Sample \#11

\begin{tabular}{|c|c|c|c|c|}
\hline $\begin{array}{l}\text { Bin } \\
\text { Range } \\
\text { (um) }\end{array}$ & $\begin{array}{l}\text { Bin Mean } \\
\text { Diameter } \\
(\mu \mathrm{m})\end{array}$ & $\begin{array}{l}\text { Diff. of the } \\
\text { log of the } \\
\text { Size }\end{array}$ & Counts & $\begin{array}{l}\text { Weighted } \\
\text { Mean } \\
\text { Diameter }\end{array}$ \\
\hline $0.30-0.49$ & 0.38 & 0.21 & 21740 & \multirow{6}{*}{1.61} \\
\hline $0.50-0.99$ & 0.70 & 0.30 & 84426 & \\
\hline $1.00-1.99$ & 1.41 & 0.30 & 38618 & \\
\hline $2.00-4.99$ & 3.16 & 0.40 & 47988 & \\
\hline $5.00-9.99$ & 7.07 & 0.30 & 3264 & \\
\hline$>10.00$ & 14.14 & 0.30 & 1555 & \\
\hline
\end{tabular}

Sample \#13

\begin{tabular}{|c|c|c|c|c|}
\hline $\begin{array}{l}\text { Bin } \\
\text { Range } \\
\text { (um) }\end{array}$ & $\begin{array}{l}\text { Bin Mean } \\
\text { Diameter } \\
(\mathrm{um})\end{array}$ & $\begin{array}{l}\text { Diff. of the } \\
\text { log of the } \\
\text { Size }\end{array}$ & Counts & $\begin{array}{l}\text { Weighted } \\
\text { Mean } \\
\text { Diameter }\end{array}$ \\
\hline $0,30-0.49$ & 0.38 & 0.21 & 23942 & \multirow{6}{*}{1.51} \\
\hline $0.50 \cdot 0.99$ & 0.70 & 0.30 & 80942 & \\
\hline $1.00-1.99$ & 1.41 & 0.30 & 34155 & \\
\hline $2.00 \cdot 4.99$ & 3.16 & 0.40 & 40036 & \\
\hline $5.00-9.99$ & 7.07 & 0.30 & 2724 & \\
\hline$>10.00$ & 14.14 & 0.30 & 1171 & \\
\hline
\end{tabular}

Samole \#12

\begin{tabular}{||c|c|c|r|r|}
\hline \begin{tabular}{|} 
Bin \\
Range \\
$(\mathrm{mm})$
\end{tabular} & $\begin{array}{l}\text { Bin Mean } \\
\text { Diameter } \\
(\mathrm{mm})\end{array}$ & $\begin{array}{l}\text { Diff. of the } \\
\text { log of the } \\
\text { Size }\end{array}$ & Counts & $\begin{array}{l}\text { Weighted } \\
\text { Mean } \\
\text { Diameter }\end{array}$ \\
\hline $0.30-0.49$ & .38 & 0.21 & 22963 & \\
\hline $0.50-0.99$ & .70 & 0.30 & 81489 & \multirow{2}{*}{1.57} \\
\hline $1.00-1.99$ & 1.41 & 0.30 & 35788 & \\
\hline $2.00-4.99$ & 3.16 & 0.40 & 43135 & \\
\hline $5.00-9.99$ & 7.07 & 0.30 & 3065 & \\
\hline$>10.00$ & 14.14 & 0.30 & 1400 & \\
\hline
\end{tabular}

Sample \#14

\begin{tabular}{|c|c|c|c|c|}
\hline $\begin{array}{l}\text { Bin } \\
\text { Range } \\
\text { (um) }\end{array}$ & $\begin{array}{l}\text { Bin Mean } \\
\text { Diameter } \\
(\mu \mathrm{m})\end{array}$ & $\begin{array}{l}\text { Diff. of the } \\
\text { log of the } \\
\text { Size }\end{array}$ & Counts & $\begin{array}{l}\text { Weighted } \\
\text { Mean } \\
\text { Diameter }\end{array}$ \\
\hline $0.30-0.49$ & .38 & 0.21 & 24169 & \multirow{6}{*}{1.51} \\
\hline $0.50-0.99$ & .70 & 0.30 & 81109 & \\
\hline $1.00-1.99$ & 1.41 & 0.30 & 33857 & \\
\hline $2.00-4.99$ & 3.16 & 0.40 & 40082 & \\
\hline $5.00-9.99$ & 7.07 & 0.30 & 2623 & \\
\hline$>10.00$ & 14.14 & 0.30 & 1242 & \\
\hline
\end{tabular}




\section{Aerosol Particle Measurement Record}

Description of sample location 3706 A Building, Resuspension Test, at $25 \mathrm{~cm}$, Date 8/12/94, Time sample started 7:32:55, Temperature 80.4 F, Humidity 40.1 \%, Dew Point $55.0 \mathrm{~F}$. Average Wind Velocity (if applicable) $.00 \mathrm{~m} / \mathrm{s}$, \# of physical barriers between sample area and outside (if applicable) 1

\begin{tabular}{|c|c|c|c|c|}
\hline $\begin{array}{l}\text { Bin } \\
\text { Range } \\
\text { (um) }\end{array}$ & \begin{tabular}{|l} 
Bin Mean \\
Diameter \\
$($ (w)
\end{tabular} & \begin{tabular}{l|} 
Diff. of the \\
log of the \\
Size
\end{tabular} & Counts & \begin{tabular}{|l|} 
Weighted \\
Mean \\
Diameter
\end{tabular} \\
\hline $0.30-0.49$ & 0.38 & 0.21 & 73482 & \multirow{6}{*}{0.72} \\
\hline $0.50-0.99$ & 0.70 & 0.30 & 32304 & \\
\hline $1.00-1.99$ & 1.41 & 0.30 & 8101 & \\
\hline $2.00-4.99$ & 3.16 & 0.40 & 6233 & \\
\hline $5.00-9.99$ & 7.07 & 0.30 & 451 & \\
\hline$>10.00$ & 14.14 & 0.30 & 126 & \\
\hline \multicolumn{5}{|l|}{ Sample \#3 } \\
\hline $\begin{array}{l}\text { Bin } \\
\text { Range } \\
(\mu \mathrm{m})\end{array}$ & \begin{tabular}{|l} 
Bin Mean \\
Diameter \\
$(u \mathrm{rm})$
\end{tabular} & $\begin{array}{l}\text { Diff. of the } \\
\text { log of the } \\
\text { Size }\end{array}$ & Counts & $\begin{array}{l}\text { Weighted } \\
\text { Mean } \\
\text { Diameter }\end{array}$ \\
\hline $0.30-0.49$ & 0.38 & 0.21 & 13184 & \multirow{6}{*}{3.54} \\
\hline $0.50-0.99$ & 0.70 & 0.30 & 32128 & \\
\hline $1.00-1.99$ & 1.41 & 0.30 & 34772 & \\
\hline $2.00-4.99$ & 3.16 & 0.40 & 105465 & \\
\hline $5.00-9.99$ & 7.07 & 0.30 & 24464 & \\
\hline$>10.00$ & 14.14 & 0.30 & 15084 & \\
\hline \multicolumn{5}{|l|}{ Sample \#5 } \\
\hline $\begin{array}{l}\text { Bin } \\
\text { Range } \\
(\mu \mathrm{m})\end{array}$ & \begin{tabular}{|l} 
Bin Mean \\
Diameter \\
(um)
\end{tabular} & $\begin{array}{l}\text { Diff. of the } \\
\text { log of the } \\
\text { Size }\end{array}$ & Counts & $\begin{array}{l}\text { Weighted } \\
\text { Mean } \\
\text { Diameter }\end{array}$ \\
\hline $0.30-0.49$ & 0.38 & 0.21 & 56282 & \multirow{6}{*}{1.13} \\
\hline $0.50 \cdot 0.99$ & $0 . \overline{70}$ & 0.30 & 32649 & \\
\hline $1.00-1.99$ & 1.41 & 0.30 & 11748 & \\
\hline $2.00 \cdot 4.99$ & 3.16 & 0.40 & 14273 & \\
\hline $5.00-9.99$ & 7.07 & 0.30 & 1867 & \\
\hline$>10.00$ & 14.14 & 0.30 & 956 & \\
\hline \multicolumn{5}{|l|}{ Sample \#7 } \\
\hline $\begin{array}{l}\text { Bin } \\
\text { Range } \\
\text { (um) }\end{array}$ & \begin{tabular}{|l} 
Bin Mean \\
Diameter \\
$(u m)$
\end{tabular} & \begin{tabular}{|l|} 
Diff. of the \\
log of the \\
Size
\end{tabular} & Counts & $\begin{array}{l}\text { Weighted } \\
\text { Mean } \\
\text { Diameter }\end{array}$ \\
\hline $0.30 \cdot 0.49$ & 0.38 & 0.21 & 61946 & \multirow{6}{*}{0.95} \\
\hline $0.50-0.99$ & 0.70 & 0.30 & 31891 & \\
\hline $1.00-1.99$ & 1.41 & 0.30 & 9906 & \\
\hline $2.00-4.99$ & 3.16 & 0.40 & 10848 & \\
\hline $5.00-9.99$ & 7.07 & 0.30 & 1237 & \\
\hline$>10.00$ & 14.14 & 0.30 & 505 & \\
\hline \multicolumn{5}{|l|}{ Sample \#9 } \\
\hline $\begin{array}{l}\text { Bin } \\
\text { Range } \\
(\mu \mathrm{m})\end{array}$ & \begin{tabular}{|l} 
Bin Mean \\
Diameter \\
$(\mu \mathrm{m})$
\end{tabular} & $\begin{array}{l}\text { Diff. of the } \\
\text { log of the } \\
\text { Size }\end{array}$ & Counts & $\begin{array}{l}\text { Woighted } \\
\text { Mean } \\
\text { Diameter }\end{array}$ \\
\hline $0.30-0.49$ & 0.38 & 0.21 & 45061 & \multirow{6}{*}{1.39} \\
\hline $0.50-0.99$ & 0.70 & 0.30 & 35059 & \\
\hline $1.00-1.99$ & 1.41 & 0.30 & 16441 & \\
\hline $2.00-4.99$ & 3.16 & 0.40 & 23439 & \\
\hline $5.00-9.99$ & 7.07 & 0.30 & 2854 & \\
\hline$>10.00$ & 14.14 & 0.30 & 905 & \\
\hline
\end{tabular}

Sample \#2

\begin{tabular}{||c|c|c|r|r||}
\hline $\begin{array}{l}\text { Bin } \\
\text { Range } \\
(\mu \mathrm{m})\end{array}$ & $\begin{array}{l}\text { Bin Mean } \\
\text { Diameter } \\
(\mathrm{em})\end{array}$ & $\begin{array}{l}\text { Diff. of the } \\
\text { log of the } \\
\text { Size }\end{array}$ & Counts & $\begin{array}{l}\text { Weighted } \\
\text { Mean } \\
\text { Diameter }\end{array}$ \\
\hline $0.30-0.49$ & .38 & 0.21 & 16098 & \\
\hline $0.50-0.99$ & .70 & 0.30 & 7904 \\
\hline $1.00-1.99$ & 1.41 & 0.30 & 3329 \\
\hline $2.00-4.99$ & 3.16 & 0.40 & 18304 \\
\hline $5.00-9.99$ & 7.07 & 0.30 & 16610 \\
\hline$>10.00$ & 14.14 & 0.30 & 190020 \\
\hline
\end{tabular}

\begin{tabular}{|c|c|c|c|c|}
\hline \multicolumn{5}{|c|}{ Sample \#4 } \\
\hline $\begin{array}{l}\text { Bin } \\
\text { Range } \\
(u \mathrm{~m})\end{array}$ & \begin{tabular}{|l} 
Bin Mean \\
Diameter \\
(emm)
\end{tabular} & $\begin{array}{l}\text { Diff. of the } \\
\log \text { of the } \\
\text { Size }\end{array}$ & Counts & $\begin{array}{l}\text { Weighted } \\
\text { Mean } \\
\text { Diameter }\end{array}$ \\
\hline $0.30-0.49$ & .38 & 0.21 & 30516 & \multirow{6}{*}{2.00} \\
\hline $0.50-0.99$ & .70 & 0.30 & 38492 & \\
\hline $1.00-1.99$ & 1.41 & 0.30 & 24285 & \\
\hline $2.00-4.99$ & 3.16 & 0.40 & 41457 & \\
\hline $5.00-9.99$ & 7.07 & 0.30 & 5945 & \\
\hline$>10.00$ & 14.14 & 0.30 & 2945 & \\
\hline
\end{tabular}
Sample $\# 6$

\begin{tabular}{|c|c|c|c|c|}
\hline $\begin{array}{l}\text { Bin } \\
\text { Range } \\
(u m)\end{array}$ & $\begin{array}{l}\text { Bin Mean } \\
\text { Diameter } \\
\text { (um) }\end{array}$ & $\begin{array}{l}\text { Diff. of the } \\
\text { log of the } \\
\text { Size }\end{array}$ & Counts & $\begin{array}{l}\text { Weighted } \\
\text { Mean } \\
\text { Diamoter }\end{array}$ \\
\hline $0.30-0.49$ & .38 & 0.21 & 64124 & \multirow{6}{*}{0.90} \\
\hline $0.50-0.99$ & .70 & 0.30 & 31201 & \\
\hline $1.00-1.99$ & 1.41 & 0.30 & 9339 & \\
\hline $2.00-4.99$ & $\overline{3.16}$ & 0.40 & 9667 & \\
\hline $5.00-9.99$ & 7.07 & 0.30 & 7035 & \\
\hline$>10.00$ & 14.14 & 0.30 & 506 & \\
\hline
\end{tabular}

\section{Sample \#8}

\begin{tabular}{|c|c|c|c|c|}
\hline $\begin{array}{l}\text { Bin } \\
\text { Range } \\
(u m)\end{array}$ & $\begin{array}{l}\text { Bin Mean } \\
\text { Diameter } \\
(\mu \mathrm{m})\end{array}$ & $\begin{array}{l}\text { Diff. of the } \\
\text { log of the } \\
\text { Size }\end{array}$ & Counts & $\begin{array}{l}\text { Weighted } \\
\text { Mean } \\
\text { Diameter }\end{array}$ \\
\hline $0.30-0.49$ & .38 & 0.21 & 52645 & \multirow{6}{*}{1.18} \\
\hline $0.50-0.99$ & .70 & 0.30 & 33832 & \\
\hline $0.00-1.99$ & 1.41 & 0.30 & 13625 & \\
\hline $2.00-4.99$ & 3.16 & 0.40 & 17182 & \\
\hline $5.00-9.99$ & 7.07 & 0.30 & 2056 & \\
\hline$>10.00$ & 14.14 & 0.30 & 663 & \\
\hline
\end{tabular}

\begin{tabular}{|c|c|c|r|r|}
\hline Sample \#10 & \multicolumn{3}{|l|}{} \\
\hline $\begin{array}{l}\text { Bin } \\
\text { Range } \\
(\mathrm{m})\end{array}$ & $\begin{array}{l}\text { Bin Mean } \\
\text { Diameter } \\
(\mathrm{mm})\end{array}$ & $\begin{array}{l}\text { Diff. of the } \\
\text { log of the } \\
\text { Size }\end{array}$ & Counts & $\begin{array}{l}\text { Weighted } \\
\text { Mean } \\
\text { Diameter }\end{array}$ \\
\hline $0.30-0.49$ & .38 & 0.21 & 40819 & \\
\hline $0.50-0.99$ & .70 & 0.30 & 35960 & \multirow{2}{*}{1.51} \\
\hline $1.00-1.99$ & 1.41 & 0.30 & 18221 & \\
\hline $2.00-4.99$ & 3.16 & 0.40 & 27807 & \\
\hline $5.00-9.99$ & 7.07 & 0.30 & 3359 & \\
\hline$>10.00$ & 14.14 & 0.30 & 1001 & \\
\hline
\end{tabular}


Sample \#11

\begin{tabular}{||l|l|l|r|r||}
\hline $\begin{array}{l}\text { Bin } \\
\text { Range } \\
(\mathrm{mm})\end{array}$ & $\begin{array}{l}\text { Bin Mean } \\
\text { Diameter } \\
(\mu \mathrm{m})\end{array}$ & $\begin{array}{l}\text { Diff. of the } \\
\text { log of the } \\
\text { Size }\end{array}$ & Counts & $\begin{array}{l}\text { Weighted } \\
\text { Mean } \\
\text { Diameter }\end{array}$ \\
\hline $0.30-0.49$ & 0.38 & 0.21 & 35693 & \\
\cline { 1 - 4 } $0.50-0.99$ & 0.70 & 0.30 & 36664 & \multirow{2}{*}{1.69} \\
\hline $1.00-1.99$ & 1.41 & 0.30 & 21043 & \\
\hline $2.00-4.99$ & 3.16 & 0.40 & 34470 & \\
\hline $5.00-9.99$ & 7.07 & 0.30 & 4472 & \\
\hline$>10.00$ & 14.14 & 0.30 & 1183 & \\
\hline
\end{tabular}

Sample \#13

\begin{tabular}{||c|c|c|r|r|}
\hline \begin{tabular}{|l|l|r||} 
Rin \\
$(\mu \mathrm{m})$
\end{tabular} & $\begin{array}{l}\text { Bin Mean } \\
\text { Diameter } \\
(\mu \mathrm{m})\end{array}$ & $\begin{array}{l}\text { Diff. of the } \\
\text { log of the } \\
\text { Size }\end{array}$ & Counts & $\begin{array}{l}\text { Weighted } \\
\text { Mean } \\
\text { Diameter }\end{array}$ \\
\hline $0.30-0.49$ & 0.38 & 0.21 & 41218 & \\
\hline $0.50-0.99$ & 0.70 & 0.30 & 34955 & \multirow{2}{*}{1.49} \\
\hline $1.00-1.99$ & 1.41 & 0.30 & 17675 & \\
\hline $2.00-4.99$ & 3.16 & 0.40 & 27106 & \\
\hline $5.00-9.99$ & 7.07 & 0.30 & 3333 & \\
\hline$>10.00$ & 14.14 & 0.30 & 880 & \\
\hline
\end{tabular}

:
Sample \#12

\begin{tabular}{|c|c|c|c|c|}
\hline $\begin{array}{l}\text { Bin } \\
\text { Range } \\
(\mu \mathrm{m})\end{array}$ & $\begin{array}{l}\text { Bin Mean } \\
\text { Diameter } \\
(\mu \mathrm{mm})\end{array}$ & $\begin{array}{l}\text { Diff. of the } \\
\log \text { of the } \\
\text { Size }\end{array}$ & Counts & $\begin{array}{l}\text { Weighted } \\
\text { Mean } \\
\text { Diameter }\end{array}$ \\
\hline $0.30-0.49$ & .38 & 0.21 & 40329 & \multirow{6}{*}{1.52} \\
\hline $0.50-0.99$ & .70 & 0.30 & 35466 & \\
\hline $1.00-1.99$ & 1.41 & 0.30 & 18266 & \\
\hline $2.00-4.99$ & 3.16 & 0.40 & 27807 & \\
\hline $5.00-9.99$ & 7.07 & 0.30 & 3473 & \\
\hline$>10.00$ & 14.14 & 0.30 & 972 & \\
\hline
\end{tabular}

Sample \#14

\begin{tabular}{||c|c|c|r|r|}
\hline \begin{tabular}{|} 
Bin \\
Range \\
$(\mu \mathrm{m})$
\end{tabular} & $\begin{array}{l}\text { Bin Mean } \\
\text { Diameter } \\
(\mathrm{\mu m})\end{array}$ & $\begin{array}{l}\text { Diff. of the } \\
\text { log of the } \\
\text { Size }\end{array}$ & Counts & $\begin{array}{l}\text { Weighted } \\
\text { Mean } \\
\text { Diameter }\end{array}$ \\
\hline $0.30-0.49$ & .38 & 0.21 & 40828 & \\
\hline $0.50-0.99$ & .70 & 0.30 & 34667 & \\
\hline $1.00-1.99$ & 1.41 & 0.30 & 17849 & \multirow{2}{*}{1.49} \\
\hline $2.00-4.99$ & 3.16 & 0.40 & 27056 & \\
\hline $5.00-9.99$ & 7.07 & 0.30 & 3303 & \\
\hline$>10.00$ & 14.14 & 0.30 & 836 & \\
\hline
\end{tabular}


Aerosol Particle Measurement Record

Description of sample location 3706 A Building, Resuspension Test, at $2.0 \mathrm{~m}$, Date 8/10/94, Time sample started 10:16:43, Temperature 76.3 F, Humidity 41.5\%, Dew Point $50.5 \mathrm{~F}$, Average Wind Velocity (if applicable) $.00 \mathrm{~m} / \mathrm{s}$, \# of physical barriers between sample area and outside (if applicable) 1

\begin{tabular}{|c|c|c|c|c|}
\hline $\begin{array}{l}\text { Bin } \\
\text { Range } \\
\text { (um) }\end{array}$ & \begin{tabular}{|l|} 
Bin Mean \\
Diameter \\
(um)
\end{tabular} & $\begin{array}{l}\text { Diff. of the } \\
\text { log of the } \\
\text { Size }\end{array}$ & Counts & $\begin{array}{l}\text { Weighted } \\
\text { Mean } \\
\text { Diameter }\end{array}$ \\
\hline $0.30-0.49$ & 0.38 & 0.21 & 32931 & \multirow{6}{*}{0.77} \\
\hline $0.50-0.99$ & 0.70 & 0.30 & 28249 & \\
\hline $1.00-1.99$ & 1.41 & 0.30 & 4685 & \\
\hline $2.00-4.99$ & 3.16 & 0.40 & 3993 & \\
\hline $5.00-9.99$ & 7.07 & 0.30 & 180 & \\
\hline$>10.00$ & 14.14 & 0.30 & 63 & \\
\hline \multicolumn{5}{|l|}{ Sample \#3 } \\
\hline $\begin{array}{l}\text { Bin } \\
\text { Range } \\
(u m)\end{array}$ & \begin{tabular}{|l|}
$\begin{array}{l}\text { Bin Mean } \\
\text { Diameter } \\
(\mu \mathrm{m})\end{array}$ \\
\end{tabular} & \begin{tabular}{|l|} 
Diff. of the \\
log of the \\
Size \\
\end{tabular} & Counts & $\begin{array}{l}\text { Weighted } \\
\text { Mean } \\
\text { Diameter }\end{array}$ \\
\hline $0.30 \cdot 0.49$ & 0.38 & 0.21 & 25122 & \multirow{6}{*}{1.40} \\
\hline $0.50 \cdot 0.99$ & 0.70 & 0.30 & 61308 & \\
\hline $1.00-1.99$ & 1.41 & 0.30 & 24216 & \\
\hline $2.00-4.99$ & 3.16 & 0.40 & 28085 & \\
\hline $5.00-9.99$ & 7.07 & 0.30 & 1686 & \\
\hline$>10.00$ & 14.14 & 0.30 & 702 & \\
\hline \multicolumn{5}{|l|}{ ample \#5 } \\
\hline $\begin{array}{l}\text { Bin } \\
\text { Range } \\
(\mu \mathrm{m}) \\
\end{array}$ & \begin{tabular}{|l|} 
Bin Mean \\
Diameter \\
$(\mu \mathrm{m})$
\end{tabular} & $\begin{array}{l}\text { Diff. of the } \\
\log \text { of the } \\
\text { Size }\end{array}$ & Counts & $\begin{array}{l}\text { Weighted } \\
\text { Mean } \\
\text { Diameter }\end{array}$ \\
\hline $0.30 \cdot 0.49$ & 0.38 & 0.21 & 16783 & \multirow{6}{*}{$\underbrace{2.17}_{*}$} \\
\hline 0.50 .0 .99 & 0.70 & 0.30 & 79631 & \\
\hline $1.00-1.99$ & 1.41 & 0.30 & 40139 & \\
\hline $2.00-4.99$ & 3.16 & 0.40 & 54338 & \\
\hline $5.00-9.99$ & 7.07 & 0.30 & 6253 & \\
\hline$>10.00$ & 14.14 & 0.30 & 7752 & \\
\hline \multicolumn{5}{|l|}{ Sample \#7 } \\
\hline \begin{tabular}{|l|} 
Bin \\
Range \\
$(\mu \mathrm{m})$ \\
\end{tabular} & \begin{tabular}{|l|} 
Bin Mean \\
Diameter \\
$(\mathrm{Lm})$
\end{tabular} & \begin{tabular}{|l|} 
Diff. of the \\
log of the \\
Size
\end{tabular} & Counts & $\begin{array}{l}\text { Weighted } \\
\text { Mean } \\
\text { Diameter }\end{array}$ \\
\hline $0.30-0.49$ & 0.38 & 0.21 & 19474 & \multirow{6}{*}{1.80} \\
\hline $0.50-0.99$ & 0.70 & 0.30 & 81037 & \\
\hline $1.00-1.99$ & 1.41 & 0.30 & 37469 & \\
\hline $2.00-4.99$ & 3.16 & 0.40 & 47344 & \\
\hline $5.00-9.99$ & 7.07 & 0.30 & 4367 & \\
\hline$>10.00$ & 14.14 & 0.30 & 3561 & \\
\hline \multicolumn{5}{|l|}{ Sample \#9 } \\
\hline \begin{tabular}{|l|} 
Bin \\
Range \\
$(\mu \mathrm{m})$ \\
\end{tabular} & \begin{tabular}{|l|} 
Bin Mean \\
Diameter \\
(um)
\end{tabular} & \begin{tabular}{|l|} 
Diff. of the \\
log of the \\
Size
\end{tabular} & Counts & $\begin{array}{l}\text { Weighted } \\
\text { Mean } \\
\text { Diameter }\end{array}$ \\
\hline $0.30 \cdot 0.49$ & 0.38 & $\begin{array}{l}0.21 \\
\end{array}$ & 23738 & \multirow{6}{*}{1.46} \\
\hline $0.50-0.99$ & 0.70 & 0.30 & 67302 & \\
\hline $1.00-1.99$ & 1.41 & 0.30 & 24182 & \\
\hline $2.00-4.99$ & 3.16 & 0.40 & 25885 & \\
\hline $5.00-9.99$ & 7.07 & 0.30 & 2205 & \\
\hline$>10.00$ & 14.14 & 0.30 & 1737 & \\
\hline
\end{tabular}

Sample \#2

\begin{tabular}{||c|c|c|r|r||}
\hline $\begin{array}{l}\text { Bin } \\
\text { Range } \\
(\mathrm{um})\end{array}$ & $\begin{array}{l}\text { Bin Mean } \\
\text { Diameter } \\
(\mathrm{um})\end{array}$ & $\begin{array}{l}\text { Diff. of the } \\
\text { log of the } \\
\text { Size }\end{array}$ & Counts & $\begin{array}{l}\text { Weighted } \\
\text { Mean } \\
\text { Diameter }\end{array}$ \\
\hline $0.30-0.49$ & .38 & 0.21 & 32307 & \\
\hline $0.50-0.99$ & .70 & 0.30 & 28777 & \multirow{2}{*}{0.78} \\
\hline $1.00-1.99$ & 1.41 & 0.30 & 4837 & \\
\hline $2.00-4.99$ & 3.16 & 0.40 & 4147 & \\
\hline $5.00-9.99$ & 7.07 & 0.30 & 195 & \\
\hline$>10.00$ & 14.14 & 0.30 & 78 & \\
\hline Sample & & &
\end{tabular}

\begin{tabular}{|c|c|c|c|c|}
\hline \multicolumn{5}{|l|}{ Sample \#4 } \\
\hline \begin{tabular}{l|} 
Bin \\
Range \\
(um)
\end{tabular} & \begin{tabular}{|l} 
Bin Mean \\
Diameter \\
$(4 \mathrm{~m})$
\end{tabular} & $\begin{array}{l}\text { Diff. of the } \\
\text { log of the } \\
\text { Size }\end{array}$ & Counts & \begin{tabular}{|l} 
Weighted \\
Mean \\
Diameter
\end{tabular} \\
\hline $0.30-0.49$ & .38 & 0.21 & 16569 & \multirow{6}{*}{2.02} \\
\hline $0.50-0.99$ & .70 & 0.30 & 83737 & \\
\hline $1.00-1.99$ & 1.41 & 0.30 & 47886 & \\
\hline $2.00-4.99$ & 3.16 & 0.40 & 68601 & \\
\hline $5.00-9.99$ & 7.07 & 0.30 & 6466 & \\
\hline$>10.00$ & $\overline{14.14}$ & 0.30 & $\overline{4716}$ & \\
\hline
\end{tabular}

Sample \#6

\begin{tabular}{|c|c|c|c|c|}
\hline $\begin{array}{l}\text { Bin } \\
\text { Range } \\
\text { (um) }\end{array}$ & \begin{tabular}{|l|} 
Bin Mean \\
Diameter \\
$(\mathrm{um})$
\end{tabular} & $\begin{array}{l}\text { Diff. of the } \\
\text { log of the } \\
\text { Size }\end{array}$ & Counts & $\begin{array}{l}\text { Weighted } \\
\text { Mean } \\
\text { Diameter }\end{array}$ \\
\hline $0.30-0.49$ & .38 & 0.21 & 18673 & \multirow{6}{*}{1.96} \\
\hline $0.50-0.99$ & .70 & 0.30 & 81348 & \\
\hline $1.00-1.99$ & 1.41 & 0.30 & 39071 & \\
\hline $2.00-4.99$ & 3.16 & 0.40 & 49853 & \\
\hline $5.00-9.99$ & 7.07 & 0.30 & 5357 & \\
\hline$>10.00$ & 14.14 & 0.30 & $\overline{5468}$ & \\
\hline
\end{tabular}

\begin{tabular}{|c|c|c|c|c|}
\hline \multicolumn{5}{|l|}{ Sample \#8 } \\
\hline \begin{tabular}{|l|} 
Bin \\
Range \\
(urm)
\end{tabular} & \begin{tabular}{|l} 
Bin Mean \\
Diameter \\
$(\mathrm{um})$
\end{tabular} & $\begin{array}{l}\text { Diff, of the } \\
\text { log of the } \\
\text { Size }\end{array}$ & Counts & $\begin{array}{l}\text { Weighted } \\
\text { Mean } \\
\text { Diamoter }\end{array}$ \\
\hline $0.30-0.49$ & .38 & 0.21 & 22110 & \multirow{6}{*}{1.60} \\
\hline 0.50 .0 .99 & .70 & 0.30 & 73979 & \\
\hline $0.00-1.99$ & 1.41 & 0.30 & 29236 & \\
\hline $2.00-4.99$ & 3.16 & 0.40 & 33402 & \\
\hline $5.00-9.99$ & 7.07 & 0.30 & 2920 & \\
\hline$>10.00$ & 14.14 & 0.30 & 2491 & \\
\hline
\end{tabular}

\begin{tabular}{|c|c|c|c|c|}
\hline \multicolumn{5}{|c|}{ Sample $\# 10$} \\
\hline $\begin{array}{l}\text { Bin } \\
\text { Range } \\
(\mathrm{erm})\end{array}$ & $\begin{array}{l}\text { Bin Mean } \\
\text { Diameter } \\
(u m)\end{array}$ & $\begin{array}{l}\text { Diff. of the } \\
\log \text { of the } \\
\text { Size }\end{array}$ & Counts & $\begin{array}{l}\text { Weighted } \\
\text { Mean } \\
\text { Diameter }\end{array}$ \\
\hline $0.30-0.49$ & .38 & 0.21 & 21616 & \multirow{6}{*}{1.56} \\
\hline $0.50-0.99$ & .70 & 0.30 & 71932 & \\
\hline $1.00-1.99$ & 1.41 & 0.30 & 28360 & \\
\hline $2.00-4.99$ & 3.16 & 0.40 & 32528 & \\
\hline $5.00-9.99$ & 7.07 & 0.30 & 2777 & \\
\hline$>10.00$ & 14.14 & 0.30 & 1975 & \\
\hline
\end{tabular}


Sample \#11

\begin{tabular}{|c|c|c|c|c|}
\hline $\begin{array}{l}\text { Bin } \\
\text { Range } \\
(\omega m)\end{array}$ & \begin{tabular}{|l|} 
Bin Mean \\
Diameter \\
$(u m)$
\end{tabular} & $\begin{array}{l}\text { Diff. of the } \\
\text { log of the } \\
\text { Size }\end{array}$ & Counts & $\begin{array}{l}\text { Weighted } \\
\text { Mean } \\
\text { Diameter }\end{array}$ \\
\hline $0.30-0.49$ & 0.38 & 0.21 & 21301 & \multirow{6}{*}{1.59} \\
\hline $0.50 \cdot 0.99$ & 0.70 & 0.30 & 74272 & \\
\hline $1.00-1.99$ & 1.41 & 0.30 & 30648 & \\
\hline $2.00-4.99$ & 3.16 & 0.40 & 35131 & \\
\hline $5.00-9.99$ & 7.07 & 0.30 & 2985 & \\
\hline$>10.00$ & 14.14 & 0.30 & 2036 & \\
\hline
\end{tabular}

Sample \#12

\begin{tabular}{|c|c|c|c|c|}
\hline \begin{tabular}{|l} 
Bin \\
Range \\
$(\mu \mathrm{m})$
\end{tabular} & \begin{tabular}{|l} 
Bin Mean \\
Diameter \\
$(u m)$
\end{tabular} & $\begin{array}{l}\text { Diff. of the } \\
\log \text { of the } \\
\text { Size }\end{array}$ & Counts & $\begin{array}{l}\text { Weighted } \\
\text { Mean } \\
\text { Diameter }\end{array}$ \\
\hline $0.30-0.49$ & .38 & 0.21 & 20509 & \multirow{6}{*}{1.62} \\
\hline $0.50-0.99$ & .70 & 0.30 & 76714 & \\
\hline $1.00-1.99$ & 1.41 & 0.30 & 33340 & \\
\hline $2.00-4.99$ & 3.16 & 0.40 & 39982 & \\
\hline $5.00-9.99$ & 7.07 & 0.30 & 3206 & \\
\hline$>10.00$ & 14.14 & 0.30 & 1881 & \\
\hline
\end{tabular}

Sample \#13

\begin{tabular}{|l|l}
\hline Bin Mean Diameter $(\mathrm{m})$ & Diff. of the log of the Size
\end{tabular}

\begin{tabular}{|r|l|}
\hline Counts & $\begin{array}{l}\text { Weighted Mean } \\
\text { Diameter }\end{array}$ \\
\hline 20737 & \\
\hline 75177 & \\
\hline 31457 & 1.60 \\
\hline 38237 & \\
\hline 3081 & \\
\hline 1817 & \\
\hline
\end{tabular}




\section{Aerosol Particle Measurement Record}

Description of sample location 3706 A Building, Resuspension Test, at $25 \mathrm{~m}$, Date 8/10/94, Time sample started_10:16:43, Temperature_76.3 F, Humidity_41.5\%, Dew Point $50.5 \mathrm{~F}$, Average Wind Velocity (if applicable) $.00 \mathrm{~m} / \mathrm{s}$, \# of physical barriers between sample area and outside (if applicable) 1

\begin{tabular}{|c|c|c|c|c|}
\hline $\begin{array}{l}\text { Bin } \\
\text { Rangs } \\
(\mu \mathrm{m})\end{array}$ & $\begin{array}{l}\text { Bin Mean } \\
\text { Diameter } \\
(\mu \mathrm{m})\end{array}$ & $\begin{array}{l}\text { Diff. of the } \\
\text { log of the } \\
\text { Size }\end{array}$ & Counts & $\begin{array}{l}\text { Weighted } \\
\text { Mean } \\
\text { Diameter }\end{array}$ \\
\hline $0.30-0.49$ & 0.38 & 0.21 & 41390 & \multirow{6}{*}{0.86} \\
\hline $0.50-0.99$ & 0.70 & 0.30 & 16694 & \\
\hline $1.00-1.99$ & 1.41 & 0.30 & 5442 & \\
\hline $2.00-4.99$ & 3.16 & 0.40 & 6178 & \\
\hline $5.00-9.99$ & 7.07 & 0.30 & 530 & \\
\hline$>10.00$ & 14.14 & 0.30 & 155 & \\
\hline \multicolumn{5}{|l|}{ Sample \#3 } \\
\hline $\begin{array}{l}\text { Bin } \\
\text { Range } \\
(\mu \mathrm{m})\end{array}$ & $\begin{array}{l}\text { Bin Mean } \\
\text { Diameter } \\
\text { (um) }\end{array}$ & $\begin{array}{l}\text { Diff. of the } \\
\text { log of the } \\
\text { Size }\end{array}$ & Counts & $\begin{array}{l}\text { Weighted } \\
\text { Mean } \\
\text { Diameter }\end{array}$ \\
\hline $0.30-0.49$ & 0.38 & 0.21 & 24548 & \multirow{6}{*}{2.13} \\
\hline $0.50-0.99$ & 0.70 & 0.30 & 32439 & \\
\hline $1.00-1.99$ & 1.41 & 0.30 & 22227 & \\
\hline $2.00-4.99$ & 3.16 & 0.40 & 41023 & \\
\hline $5.00-9.99$ & 7.07 & 0.30 & 6003 & \\
\hline$>10.00$ & 14.14 & 0.30 & 2769 & \\
\hline \multicolumn{5}{|l|}{ Sample \#5 } \\
\hline \begin{tabular}{|l} 
Bin \\
Range \\
$(\mu \mathrm{m})$
\end{tabular} & $\begin{array}{l}\text { Bin Mean } \\
\text { Diameter } \\
(\mu \mathrm{m})\end{array}$ & $\begin{array}{l}\text { Diff. of the } \\
\text { log of the } \\
\text { Size }\end{array}$ & Counts & $\begin{array}{l}\text { Weighted } \\
\text { Mean } \\
\text { Diameter }\end{array}$ \\
\hline $0.30-0.49$ & 0.38 & 0.21 & 36480 & \multirow{6}{*}{1.22} \\
\hline $0.50-0.99$ & 0.70 & 0.30 & 19905 & \\
\hline $1.00-1.99$ & 1.41 & 0.30 & 8260 & \\
\hline $2.00-4.99$ & 3.16 & $0.40-$ & 11306 & \\
\hline $5.00-9.99$ & 7.07 & 0.30 & 1502 & \\
\hline$>10.00$ & 14.14 & 0.30 & 703 & \\
\hline \multicolumn{5}{|l|}{ Sample \#7 } \\
\hline $\begin{array}{l}\text { Bin } \\
\text { Range } \\
(\mu \mathrm{m})\end{array}$ & $\begin{array}{l}\text { Bin Mean } \\
\text { Diameter } \\
(\mu \mathrm{m})\end{array}$ & $\begin{array}{l}\text { Diff. of the } \\
\text { log of the } \\
\text { Size }\end{array}$ & Counts & $\begin{array}{l}\text { Weighted } \\
\text { Mean } \\
\text { Diameter }\end{array}$ \\
\hline $0.30-0.49$ & 0.38 & 0.21 & 30528 & \multirow{6}{*}{1.63} \\
\hline $0.50 \cdot 0.99$ & 0.70 & 0.30 & 26936 & \\
\hline $1.00-1.99$ & 1.41 & 0.30 & 14782 & \\
\hline $2.00 \cdot 4.99$ & 3.16 & 0.40 & 23969 & \\
\hline $5.00-9.99$ & 7.07 & 0.30 & 3042 & \\
\hline$>10.00$ & 14.14 & 0.30 & 1063 & \\
\hline \multicolumn{5}{|l|}{ Sample \#9 } \\
\hline $\begin{array}{l}\text { Bin } \\
\text { Range } \\
\text { (um) }\end{array}$ & \begin{tabular}{|l|} 
Bin Mean \\
Diameter \\
$($ ( m)
\end{tabular} & $\begin{array}{l}\text { Diff. of the } \\
\text { log of the } \\
\text { Size }\end{array}$ & Counts & $\begin{array}{l}\text { Weighted } \\
\text { Mean } \\
\text { Diameter }\end{array}$ \\
\hline $0,30-0.49$ & 0.38 & 0.21 & 21951 & \multirow{6}{*}{2.18} \\
\hline $0.50-0.99$ & $0 . \overline{70}$ & 0.30 & 36596 & \\
\hline $1.00-1.99$ & 1.41 & 0.30 & 27366 & \\
\hline $2.00-4.99$ & 3.16 & 0.40 & 52347 & \\
\hline $5.00-9.99$ & 7.07 & 0.30 & 7442 & \\
\hline$>10.00$ & 14.14 & 0.30 & 2310 & \\
\hline
\end{tabular}

\begin{tabular}{|c|c|c|c|c|}
\hline $\begin{array}{l}\text { Bin } \\
\text { Range } \\
\text { (um) }\end{array}$ & \begin{tabular}{|l|} 
Bin Mean \\
Diameter \\
$(\mathrm{(m)})$
\end{tabular} & $\begin{array}{l}\text { Diff. of the } \\
\log \text { of the } \\
\text { Size }\end{array}$ & Counts & $\begin{array}{l}\text { Woighted } \\
\text { Mean } \\
\text { Diameter }\end{array}$ \\
\hline $0.30-0.49$ & .38 & 0.21 & 7801 & \\
\hline $0.50-0.99$ & .70 & 0.30 & 6384 & \\
\hline $1.00-1.99$ & 1.41 & 0.30 & 5197 & \\
\hline $2.00-4.99$ & 3.16 & 0.40 & 33853 & \\
\hline $5.00-9.99$ & 7.07 & 0.30 & 28126 & \\
\hline$>10.00$ & 14.14 & 0.30 & 179259 & \\
\hline \multicolumn{5}{|l|}{ Sample \#4 } \\
\hline $\begin{array}{l}\text { Bin } \\
\text { Range } \\
\text { (lm) }\end{array}$ & \begin{tabular}{|l} 
Bin Mean \\
Diameter \\
$(\mathrm{um})$
\end{tabular} & \begin{tabular}{|l} 
Diff. of the \\
log of the \\
Size
\end{tabular} & Counts & $\begin{array}{l}\text { Weighted } \\
\text { Mean } \\
\text { Diameter }\end{array}$ \\
\hline $0.30-0.49$ & .38 & 0.21 & 28402 & \multirow{6}{*}{1.75} \\
\hline $0.50-0.99$ & .70 & 0.30 & 28682 & \\
\hline $1.00-1.99$ & 1.41 & 0.30 & 16692 & \\
\hline $2.00-4.99$ & 3.16 & 0.40 & 26837 & \\
\hline $5.00-9.99$ & 7.07 & 0.30 & 3484 & \\
\hline$>10.00$ & 14.14 & 0.30 & 1440 & \\
\hline \multicolumn{5}{|l|}{ Sample \#6 } \\
\hline $\begin{array}{l}\text { Bin } \\
\text { Range } \\
(\mu \mathrm{m})\end{array}$ & $\begin{array}{l}\text { Bin Mean } \\
\text { Diameter } \\
(\mu \mathrm{m})\end{array}$ & $\begin{array}{l}\text { Diff. of the } \\
\text { log of the } \\
\text { Size }\end{array}$ & Counts & $\begin{array}{l}\text { Woighted } \\
\text { Mean } \\
\text { Diameter }\end{array}$ \\
\hline $0.30-0.49$ & .38 & 0.21 & 37737 & \multirow{6}{*}{ 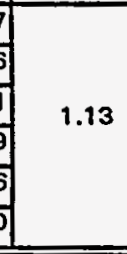 } \\
\hline $0.50-0.99$ & .70 & 0.30 & 19136 & \\
\hline $1.00-1.99$ & 1.41 & 0.30 & 7711 & \\
\hline $2.00-4.99$ & 3.16 & 0.40 & 10239 & \\
\hline $5.00-9.99$ & 7.07 & 0.30 & 1176 & \\
\hline$>10.00$ & 14.14 & 0.30 & 500 & \\
\hline \multicolumn{5}{|l|}{ Sample $\$ 8$} \\
\hline $\begin{array}{l}\text { Bin } \\
\text { Range } \\
(\mathrm{mm})\end{array}$ & $\begin{array}{l}\text { Bin Mean } \\
\text { Diameter } \\
(\mu \mathrm{m})\end{array}$ & \begin{tabular}{|l} 
Diff. of the \\
log of the \\
Size \\
\end{tabular} & Counts & \begin{tabular}{|l} 
Weighted \\
Mean \\
Diameter
\end{tabular} \\
\hline $0.30-0.49$ & .38 & 0.21 & 24924 & \multirow{6}{*}{1.94} \\
\hline $0.50-0.99$ & .70 & 0.30 & 33107 & \\
\hline $0.00-1.99$ & 1.41 & 0.30 & 22077 & \\
\hline $2.00-4.99$ & 3.16 & 0.40 & 38364 & \\
\hline $5.00-9.99$ & 7.07 & 0.30 & 5048 & \\
\hline$>10.00$ & 14.14 & 0.30 & 1592 & \\
\hline \multicolumn{5}{|c|}{ Sample \#10 } \\
\hline \begin{tabular}{l|} 
Bin \\
Range \\
$(\mu \mathrm{m})$
\end{tabular} & \begin{tabular}{|l} 
Bin Mean \\
Diameter \\
$(\mu \mathrm{m})$
\end{tabular} & $\begin{array}{l}\text { Diff. of the } \\
\text { log of the } \\
\text { Size }\end{array}$ & Counts & $\begin{array}{l}\text { Weighted } \\
\text { Mean } \\
\text { Diamoter }\end{array}$ \\
\hline $0.30-0.49$ & .38 & 0.21 & 21309 & \multirow{6}{*}{2.23} \\
\hline $0.50-0.99$ & .70 & 0.30 & 36528 & \\
\hline $1.00-1.99$ & 1.41 & 0.30 & 28261 & \\
\hline $2.00-4.99$ & 3.16 & 0.40 & 55871 & \\
\hline $5.00-9.99$ & 7.07 & 0.30 & 8064 & \\
\hline$>10.00$ & 14.14 & 0.30 & 2305 & \\
\hline
\end{tabular}


Sample \#11

\begin{tabular}{|c|c|c|c|c|}
\hline $\begin{array}{l}\text { Bin } \\
\text { Range } \\
(\mathrm{um})\end{array}$ & \begin{tabular}{|l} 
Bin Mean \\
Diameter \\
(uml
\end{tabular} & $\begin{array}{l}\text { Diff. of the } \\
\log \text { of the } \\
\text { Size }\end{array}$ & Counts & \begin{tabular}{|l|} 
Weighted \\
Mean \\
Diameter
\end{tabular} \\
\hline $0.30-0.49$ & 0.38 & 0.21 & 21214 & \multirow{6}{*}{2.20} \\
\hline $0.50-0.99$ & 0.70 & 0.30 & 36627 & \\
\hline $1.00-1.99$ & 1.41 & 0.30 & 27893 & \\
\hline $2.00-4.99$ & 3.16 & 0.40 & 54110 & \\
\hline $5.00-9.99$ & 7.07 & 0.30 & 7823 & \\
\hline$>10.00$ & 14.14 & 0.30 & 2129 & \\
\hline
\end{tabular}

Sample \#13
Samole \#12

\begin{tabular}{||c|c|c|r|r||}
\hline $\begin{array}{l}\text { Bin } \\
\text { Range } \\
(\mu \mathrm{m})\end{array}$ & $\begin{array}{l}\text { Bin Mean } \\
\text { Diameter } \\
(\mu \mathrm{m})\end{array}$ & $\begin{array}{l}\text { Diff. of the } \\
\text { log of the } \\
\text { Size }\end{array}$ & Counts & $\begin{array}{l}\text { Weighted } \\
\text { Mean } \\
\text { Diameter }\end{array}$ \\
\hline $0.30-0.49$ & .38 & 0.21 & 22713 & \\
\cline { 1 - 4 } $0.50-0.99$ & .70 & 0.30 & 34705 & \multirow{2}{*}{2.08} \\
\cline { 1 - 4 } $1.00-1.99$ & 1.41 & 0.30 & 24942 \\
\cline { 1 - 4 } $2.00-4.99$ & 3.16 & 0.40 & 46205 & \\
\hline $5.00-9.99$ & 7.07 & 0.30 & 6346 & \\
\hline$>10.00$ & 14.14 & 0.30 & 1761 & \\
\hline
\end{tabular}

$(\mu \mathrm{m})$

\begin{tabular}{|c|c|c|c|}
\hline 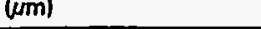 & & & \\
\hline .38 & 0.21 & 24014 & \multirow{6}{*}{1.95} \\
\hline .70 & 0.30 & 32504 & \\
\hline 1.41 & 0.30 & 21677 & \\
\hline 3.16 & 0.40 & 38718 & \\
\hline 7.07 & 0.30 & 5051 & \\
\hline 14.14 & 0.30 & 1393 & \\
\hline
\end{tabular}




\section{Aerosol Particle Measurement Record}

Description of sample location 3706A Building, Resuspension Test, at $2.0 \mathrm{~m}$, Date 8/10/94, Time sample started 11:33:41, Temperature 78.5 F, Humidity $38.8 \%$, Dew Point 51.0 F, Average Wind Velocity (if applicable) $.02 \mathrm{~m} / \mathrm{s}$, \# of physical barriers between sample area and outside (if applicable) 1

\begin{tabular}{|c|c|c|c|c|}
\hline $\begin{array}{l}\text { Bin } \\
\text { Range } \\
\text { (um) }\end{array}$ & $\begin{array}{l}\text { Bin Mean } \\
\text { Diameter } \\
(\mathrm{erm})\end{array}$ & \begin{tabular}{|l|} 
Diff. of the \\
log of the \\
Size
\end{tabular} & Counts & \begin{tabular}{|l} 
Weighted \\
Mean \\
Diameter
\end{tabular} \\
\hline $0,30-0.49$ & 0.38 & 0.21 & 30646 & \multirow{6}{*}{0.94} \\
\hline $0.50-0.99$ & 0.70 & 0.30 & 38082 & \\
\hline $1.00-1.99$ & 1.41 & 0.30 & 9075 & \\
\hline $2.00-4.99$ & 3.16 & 0.40 & 8248 & \\
\hline $5.00-9.99$ & 7.07 & 0.30 & 368 & \\
\hline$>10.00$ & 14.14 & 0.30 & 108 & \\
\hline \multicolumn{5}{|l|}{ Sample \#3 } \\
\hline $\begin{array}{l}\text { Bin } \\
\text { Range } \\
(\mu \mathrm{m})\end{array}$ & \begin{tabular}{|l|} 
Bin Mean \\
Diameter \\
$(\mu \mathrm{m})$
\end{tabular} & $\begin{array}{l}\text { Diff. of the } \\
\text { log of the } \\
\text { Size }\end{array}$ & Counts & $\begin{array}{l}\text { Weighted } \\
\text { Mean } \\
\text { Diameter }\end{array}$ \\
\hline $0.30-0.49$ & 0.38 & 0.21 & 30323 & \multirow{6}{*}{0.98} \\
\hline $0.50-0.99$ & 0.70 & 0.30 & 38926 & \\
\hline $1.00-1.99$ & 1.41 & 0.30 & 9240 & \\
\hline $2.00-4.99$ & 3.16 & 0.40 & 8628 & \\
\hline $5.00-9.99$ & 7.07 & 0.30 & 464 & \\
\hline$>10.00$ & 14.14 & 0.30 & 258 & \\
\hline \multicolumn{5}{|l|}{ Sample \#5 } \\
\hline $\begin{array}{l}\text { Bin } \\
\text { Range } \\
(\mu \mathrm{m})\end{array}$ & $\begin{array}{l}\text { Bin Mean } \\
\text { Diameter } \\
(\mu \mathrm{m})\end{array}$ & \begin{tabular}{|l|} 
Diff. of the \\
log of the \\
Size
\end{tabular} & Counts & $\begin{array}{l}\text { Weighted } \\
\text { Mean } \\
\text { Diameter }\end{array}$ \\
\hline $0.30 \cdot 0.49$ & \begin{tabular}{|l}
0.38 \\
\end{tabular} & 0.21 & 25500 & \multirow{6}{*}{1.54} \\
\hline $0.50-0.99$ & 0.70 & 0.30 & 65501 & \\
\hline $1.00-1.99$ & 1.41 & 0.30 & 20366 & \\
\hline $2.00-4.99$ & 3.16 & 0.40 & 21671 & \\
\hline $5.00-9.99$ & 7.07 & 0.30 & 2258 & \\
\hline$>10.00$ & 14.14 & 0.30 & 3109 & \\
\hline \multicolumn{5}{|l|}{ Sample \#7 } \\
\hline $\begin{array}{l}\text { Bin } \\
\text { Range } \\
\text { uml }) \\
\end{array}$ & \begin{tabular}{|l|} 
Bin Mean \\
Diameter \\
$(\mu \mathrm{m})$
\end{tabular} & $\begin{array}{l}\text { Diff. of the } \\
\log \text { of the } \\
\text { Size }\end{array}$ & Counts & $\begin{array}{l}\text { Weighted } \\
\text { Mean } \\
\text { Diameter }\end{array}$ \\
\hline $0.30-0.49$ & 0.38 & \begin{tabular}{|l|}
0.21 \\
\end{tabular} & 24989 & \multirow{6}{*}{1.54} \\
\hline $0.50-0.99$ & 0.70 & 0.30 & 66501 & \\
\hline $1.00-1.99$ & 1.41 & 0.30 & 20843 & \\
\hline $2.00-4.99$ & 3.16 & 0.40 & 22663 & \\
\hline $5.00-9.99$ & 7.07 & 0.30 & 2399 & \\
\hline$>10.00$ & 14.14 & 0.30 & 3029 & \\
\hline \multicolumn{5}{|l|}{ Sample \#9 } \\
\hline $\begin{array}{l}\text { Bin } \\
\text { Range } \\
\text { (um) }\end{array}$ & \begin{tabular}{|l} 
Bin Mean \\
Diameter \\
$(u m)$
\end{tabular} & \begin{tabular}{|l|} 
Diff. of the \\
log of the \\
Size
\end{tabular} & Counts & $\begin{array}{l}\text { Weighted } \\
\text { Mean } \\
\text { Diameter }\end{array}$ \\
\hline $0.30-0.49$ & 0.38 & 0.21 & 27101 & \multirow{6}{*}{1.34} \\
\hline $0.50-0.99$ & 0.70 & 0.30 & 57505 & \\
\hline $1.00-1.99$ & 1.41 & 0.30 & 15946 & \\
\hline $2.00 \cdot 4.99$ & 3.16 & 0.40 & 16395 & \\
\hline $5.00-9.99$ & 7.07 & 0.30 & 1530 & \\
\hline$>10.00$ & 14.14 & 0.30 & 1837 & \\
\hline
\end{tabular}

Sample \#2

\begin{tabular}{|c|c|c|c|c|}
\hline $\begin{array}{l}\text { Bin } \\
\text { Range } \\
(u m)\end{array}$ & \begin{tabular}{|l} 
Bin Mean \\
Diameter \\
$(\mathrm{um})$
\end{tabular} & $\begin{array}{l}\text { Diff. of the } \\
\text { log of the } \\
\text { Size }\end{array}$ & Counts & $\begin{array}{l}\text { Weighted } \\
\text { Mean } \\
\text { Diameter }\end{array}$ \\
\hline $0.30-0.49$ & .38 & 0.21 & $\overline{30314}$ & \multirow{6}{*}{0.99} \\
\hline $0.50-0.99$ & .70 & 0.30 & 38918 & \\
\hline $1.00-1.99$ & 1.41 & 0.30 & 9065 & \\
\hline $2.00-4.99$ & 3.16 & 0.40 & 8262 & \\
\hline $5.00-9.99$ & 7.07 & 0.30 & 471 & \\
\hline$>10.00$ & 14.14 & 0.30 & 367 & \\
\hline
\end{tabular}

Sample \#4

\begin{tabular}{|c|c|c|c|c|}
\hline \begin{tabular}{|l} 
Bin \\
Range \\
(um)
\end{tabular} & \begin{tabular}{|l} 
Bin Mean \\
Diameter \\
$(\mathrm{(mm)}$
\end{tabular} & $\begin{array}{l}\text { Diff. of the } \\
\text { log of the } \\
\text { Size }\end{array}$ & Counts & \begin{tabular}{|l|} 
Weighted \\
Mean \\
Diameter
\end{tabular} \\
\hline $0.30-0.49$ & .38 & 0.21 & 19591 & \\
\hline $0.50-0.99$ & .70 & 0.30 & 75809 & \\
\hline $1.00-1.99$ & 1.41 & 0.30 & 32645 & \\
\hline $2.00-4.99$ & 3.16 & 0.40 & 41129 & \\
\hline $5.00-9.99$ & 7.07 & 0.30 & 4749 & \\
\hline$>10.00$ & 14.14 & 0.30 & 6033 & \\
\hline
\end{tabular}

Sample \#6

\begin{tabular}{|c|c|c|c|c|}
\hline $\begin{array}{l}\text { Bin } \\
\text { Range } \\
\text { (um) }\end{array}$ & $\begin{array}{l}\text { Bin Mean } \\
\text { Diameter } \\
(\mu \mathrm{m})\end{array}$ & $\begin{array}{l}\text { Diff. of the } \\
\log \text { of the } \\
\text { Size }\end{array}$ & Counts & $\begin{array}{l}\text { Weighted } \\
\text { Mean } \\
\text { Diameter }\end{array}$ \\
\hline $0.30-0.49$ & .38 & 0.21 & 22762 & \multirow{6}{*}{1.77} \\
\hline $0.50-0.99$ & .70 & 0.30 & 71952 & \\
\hline $1.00-1.99$ & 1.41 & 0.30 & 24891 & \\
\hline $2.00-4.99$ & 3.16 & 0.40 & 28334 & \\
\hline $5.00-9.99$ & 7.07 & 0.30 & 3299 & \\
\hline$>10.00$ & 14.14 & 0.30 & 4873 & \\
\hline
\end{tabular}

Sample \#8

\begin{tabular}{|c|c|c|c|c|}
\hline $\begin{array}{l}\text { Bin } \\
\text { Range } \\
(\omega \mathrm{m})\end{array}$ & \begin{tabular}{|l|} 
Bin Mean \\
Diameter \\
$(\mu \mathrm{m})$
\end{tabular} & $\begin{array}{l}\text { Diff. of the } \\
\log \text { of the } \\
\text { Size }\end{array}$ & Counts & $\begin{array}{l}\text { Weighted } \\
\text { Mean } \\
\text { Diameter }\end{array}$ \\
\hline $0.30-0.49$ & .38 & 0.21 & 32480 & \multirow{6}{*}{1.20} \\
\hline $0.50-0.99$ & .70 & 0.30 & 54456 & \\
\hline $0.00-1.99$ & 1.41 & 0.30 & 13299 & \\
\hline $2.00-4.99$ & 3.16 & 0.40 & 13418 & \\
\hline $5.00-9.99$ & 7.07 & 0.30 & 1084 & \\
\hline$>10.00$ & 14.14 & 0.30 & 1388 & \\
\hline
\end{tabular}

\begin{tabular}{|c|c|c|c|c|}
\hline \multicolumn{5}{|c|}{ Sample \#10 } \\
\hline \begin{tabular}{|l} 
Bin \\
Range \\
$(u \mathrm{~m})$
\end{tabular} & \begin{tabular}{|l|} 
Bin Mean \\
Diameter \\
$(\mu \mathrm{m})$
\end{tabular} & $\begin{array}{l}\text { Diff. of the } \\
\log \text { of the } \\
\text { Size }\end{array}$ & Counts & $\begin{array}{l}\text { Weighted } \\
\text { Mean } \\
\text { Diameter }\end{array}$ \\
\hline $0.30-0.49$ & .38 & 0.21 & 26640 & \multirow{6}{*}{1.36} \\
\hline $0.50-0.99$ & .70 & 0.30 & 57789 & \\
\hline $1.00-1.99$ & 1.41 & 0.30 & 16945 & \\
\hline $2.00-4.99$ & 3.16 & 0.40 & 17779 & \\
\hline $5.00-9.99$ & 7.07 & 0.30 & 1624 & \\
\hline$>10.00$ & 14.14 & 0.30 & 1716 & \\
\hline
\end{tabular}


WHC-MR-0496

Sample \#11

\begin{tabular}{|c|c|c|c|c|}
\hline $\begin{array}{l}\text { Bin } \\
\text { Range } \\
(\mu \mathrm{m})\end{array}$ & \begin{tabular}{|l} 
Bin Mean \\
Diameter \\
$(\mu \mathrm{m})$
\end{tabular} & $\begin{array}{l}\text { Diff. of the } \\
\log \text { of the } \\
\text { Size }\end{array}$ & Counts & \begin{tabular}{|l|} 
Weighted \\
Mean \\
Diameter
\end{tabular} \\
\hline $0.30-0.49$ & 0.38 & 0.21 & 25462 & \multirow{2}{*}{-} \\
\hline $0.50-0.99$ & 0.70 & 0.30 & 58983 & \\
\hline $1.00-1.99$ & 1.41 & 0.30 & 17989 & \multirow{4}{*}{1.38} \\
\hline $2.00-4.99$ & 3.16 & 0.40 & 18945 & \\
\hline $5.00-9.99$ & 7.07 & 0.30 & 1633 & \\
\hline$>10.00$ & 14.14 & 0.30 & 1734 & \\
\hline
\end{tabular}

Samole \#12

\begin{tabular}{|c|c|c|c|c|}
\hline $\begin{array}{l}\text { Bin } \\
\text { Range } \\
(\mu \mathrm{m})\end{array}$ & \begin{tabular}{|l|} 
Bin Mean \\
Diameter \\
$(\mu \mathrm{m})$
\end{tabular} & $\begin{array}{l}\text { Diff. of the } \\
\text { log of the } \\
\text { Size }\end{array}$ & Counts & $\begin{array}{l}\text { Weighted } \\
\text { Mean } \\
\text { Diameter }\end{array}$ \\
\hline $0.30-0.49$ & .38 & 0.21 & 26189 & \multirow{6}{*}{1.32} \\
\hline $0.50-0.99$ & .70 & 0.30 & $\overline{57008}$ & \\
\hline $1.00-1.99$ & 1.41 & 0.30 & 17161 & \\
\hline $2.00-4.99$ & 3.16 & 0.40 & 18260 & \\
\hline $5.00-9.99$ & 7.07 & 0.30 & 1580 & \\
\hline$>10.00$ & 14.14 & 0.30 & 1232 & \\
\hline
\end{tabular}




\section{Aerosol Particle Measurement Reċord}

Description of sample location_3706A Building, Resuspension Test, at $.25 \mathrm{~m}$, Date 8/10/94, Time sample started $11: 33: 41$, Temperature $78.5 \mathrm{~F}$, Humidity $38.8 \%$, Dew Point $51.0 \mathrm{~F}$, Average Wind Velocity (if applicable) $.02 \mathrm{~m} / \mathrm{s}$, \# of physical barriers between sample area and outside (if applicable) 1

\begin{tabular}{|c|c|c|c|c|}
\hline $\begin{array}{l}\text { Bin } \\
\text { Range } \\
\text { (um) }\end{array}$ & $\begin{array}{l}\text { Bin Mean } \\
\text { Diameter } \\
(\mu \mathrm{m})\end{array}$ & $\begin{array}{l}\text { Diff, of the } \\
\log \text { of the } \\
\text { Size }\end{array}$ & Counts & $\begin{array}{l}\text { Weighted } \\
\text { Mean } \\
\text { Diameter }\end{array}$ \\
\hline $0.30-0.49$ & 0.38 & 0.21 & 38701 & \multirow{6}{*}{1.08} \\
\hline $0.50-0.99$ & 0.70 & 0.30 & 21011 & \\
\hline $1.00 \cdot 1.99$ & 1.41 & 0.30 & 8736 & \\
\hline $2.00-4.99$ & 3.16 & 0.40 & 10952 & \\
\hline $5.00-9.99$ & 7.07 & 0.30 & 1036 & \\
\hline$>10.00$ & 14.14 & 0.30 & 245 & \\
\hline \multicolumn{5}{|l|}{ Samole \#3 } \\
\hline $\begin{array}{l}\text { Bin } \\
\text { Range } \\
(\mathrm{um}) \\
\end{array}$ & $\begin{array}{l}\text { Bin Mean } \\
\text { Diameter } \\
(u \mathrm{~m}) \\
\end{array}$ & $\begin{array}{l}\text { Diff. of the } \\
\text { log of the } \\
\text { Size }\end{array}$ & Counts & $\begin{array}{l}\text { Weighted } \\
\text { Mean } \\
\text { Diameter }\end{array}$ \\
\hline $0.30-0.49$ & 0.38 & 0.21 & 8249 & \multirow{6}{*}{4.68} \\
\hline $0.50 \cdot 0.99$ & 0.70 & 0.30 & 22387 & \\
\hline $1.00-1.99$ & 1.41 & 0.30 & 26952 & \\
\hline $2.00-4.99$ & 3.16 & 0.40 & 110160 & \\
\hline $5.00-9.99$ & 7.07 & 0.30 & 36038 & \\
\hline$>10.00$ & 14.14 & 0.30 & 31127 & \\
\hline \multicolumn{5}{|l|}{ Sample \#5 } \\
\hline \begin{tabular}{|l} 
Bin \\
Range \\
(um)
\end{tabular} & $\begin{array}{l}\text { Bin Mean } \\
\text { Diameter } \\
(\mu \mathrm{m})\end{array}$ & $\begin{array}{l}\text { Diff. of the } \\
\text { log of the } \\
\text { Size }\end{array}$ & Counts & $\begin{array}{l}\text { Weighted } \\
\text { Mean } \\
\text { Diameter }\end{array}$ \\
\hline $0.30 \cdot 0.49$ & 0.38 & 0.21 & 34561 & \multirow{6}{*}{1.39} \\
\hline $0.50 \cdot 0.99$ & 0.70 & 0.30 & 24669 & \\
\hline $1.00-1.99$ & 1.41 & 0.30 & 11746 & \\
\hline $2.00-4.99$ & 3.16 & 0.40 & 16177 & \\
\hline $5.00-9.99$ & 7.07 & 0.30 & 1927 & \\
\hline$>10.00$ & 14.14 & 0.30 & 964 & \\
\hline \multicolumn{5}{|l|}{ Sample \#7 } \\
\hline $\begin{array}{l}\text { Bin } \\
\text { Range } \\
(\mu \mathrm{m}) \\
\end{array}$ & $\begin{array}{l}\text { Bin Mean } \\
\text { Diameter } \\
(\mu \mathrm{m})\end{array}$ & $\begin{array}{l}\text { Diff. of the } \\
\text { log of the } \\
\text { Size }\end{array}$ & Counts & $\begin{array}{l}\text { Weighted } \\
\text { Mean } \\
\text { Diameter }\end{array}$ \\
\hline $0.30-0.49$ & 0.38 & 0.21 & 34828 & \multirow{6}{*}{$\begin{array}{l}1.38 \\
\text {. }\end{array}$} \\
\hline $0.50-0.99$ & 0.70 & 0.30 & 25030 & \\
\hline $1.00-1.99$ & 1.41 & 0.30 & 11872 & \\
\hline $2.00-4.99$ & 3.16 & 0.40 & 15766 & \\
\hline $5.00-9.99$ & 7.07 & 0.30 & 1859 & \\
\hline$>10.00$ & 14.14 & 0.30 & 977 & \\
\hline \multicolumn{5}{|l|}{ Sample \#9 } \\
\hline $\begin{array}{l}\text { Bin } \\
\text { Range } \\
\text { (um) } \\
\end{array}$ & $\begin{array}{l}\text { Bin Mean } \\
\text { Diameter } \\
(\mu \mathrm{m})\end{array}$ & $\begin{array}{l}\text { Diff. of the } \\
\text { log of the } \\
\text { Size }\end{array}$ & Counts & $\begin{array}{l}\text { Weighted } \\
\text { Mean } \\
\text { Diameter }\end{array}$ \\
\hline $0.30-0.49$ & 0.38 & 0.21 & 28952 & \multirow{6}{*}{1.67} \\
\hline $0.50-0.99$ & 0.70 & 0.30 & 28189 & \\
\hline $1.00-1.99$ & 1.41 & 0.30 & 15949 & \\
\hline $2.00-4.99$ & 3.16 & 0.40 & 24724 & \\
\hline $5.00-9.99$ & 7.07 & 0.30 & 3283 & \\
\hline$>10.00$ & 14.14 & 0.30 & 1148 & \\
\hline
\end{tabular}

\begin{tabular}{|c|c|c|c|c|}
\hline $\begin{array}{l}\text { Bin } \\
\text { Range } \\
\text { (em) }\end{array}$ & $\begin{array}{l}\text { Bin Mean } \\
\text { Diameter } \\
(\mu \mathrm{m})\end{array}$ & $\begin{array}{l}\text { Diff. of the } \\
\log \text { of the } \\
\text { Size }\end{array}$ & Counts & $\begin{array}{l}\text { Weighted } \\
\text { Mean } \\
\text { Diamoter }\end{array}$ \\
\hline $0.30-0.49$ & .38 & 0.21 & 12231 & \\
\hline $0.50-0.99$ & .70 & 0.30 & 23071 & \\
\hline $1.00-1.99$ & 1.41 & 0.30 & 21221 & \\
\hline $2.00-4.99$ & 3.16 & 0.40 & 83426 & \\
\hline $5.00-9.99$ & 7.07 & 0.30 & 37092 & \\
\hline$>10.00$ & 14.14 & 0.30 & 41988 & \\
\hline \multicolumn{5}{|l|}{ Sample \#4 } \\
\hline \begin{tabular}{|l|} 
Bin \\
Range \\
(um) \\
\end{tabular} & $\begin{array}{l}\text { Bin Mean } \\
\text { Diameter } \\
(\mu \mathrm{m})\end{array}$ & $\begin{array}{l}\text { Diff. of the } \\
\text { log of the } \\
\text { Size }\end{array}$ & Counts & $\begin{array}{l}\text { Weighted } \\
\text { Mean } \\
\text { Diameter }\end{array}$ \\
\hline $0.30-0.49$ & .38 & 0.21 & 23430 & \multirow{6}{*}{2.31} \\
\hline $0.50-0.99$ & .70 & 0.30 & 38151 & \\
\hline $1.00-1.99$ & 1.41 & 0.30 & 27358 & \\
\hline $2.00-4.99$ & 3.16 & 0.40 & 48710 & \\
\hline 5.00-9.99. & 7.07 & 0.30 & 7217 & \\
\hline$>10.00$ & 14.14 & 0.30 & 4687 & \\
\hline \multicolumn{5}{|c|}{ Sample $\# 6$} \\
\hline \begin{tabular}{|l|} 
Bin \\
Range \\
$(\mu \mathrm{m})$
\end{tabular} & $\begin{array}{l}\text { Bin Mean } \\
\text { Diameter } \\
(\mu \mathrm{m})\end{array}$ & $\begin{array}{l}\text { Diff. of the } \\
\log \text { of the } \\
\text { Size }\end{array}$ & Counts & $\begin{array}{l}\text { Weighted } \\
\text { Mean } \\
\text { Diameter }\end{array}$ \\
\hline $0.30-0.49$ & .38 & 0.21 & 37249 & \multirow{6}{*}{1.22} \\
\hline $0.50-0.99$ & .70 & 0.30 & 22716 & \\
\hline $1.00-1.99$ & 1.41 & 0.30 & 10154 & \\
\hline $2.00-4.99$ & 3.16 & 0.40 & 13044 & \\
\hline $5.00-9.99$ & 7.07 & 0.30 & 1438 & \\
\hline$>10.00$ & 14.14 & 0.30 & 592 & \\
\hline \multicolumn{5}{|c|}{ Sample $\# 8$} \\
\hline \begin{tabular}{|l|} 
Bin \\
Range \\
(um) \\
\end{tabular} & $\begin{array}{l}\text { Bin Mean } \\
\text { Diameter } \\
(\mu \mathrm{m})\end{array}$ & $\begin{array}{l}\text { Diff. of the } \\
\log \text { of the } \\
\text { Size }\end{array}$ & Counts & $\begin{array}{l}\text { Weighted } \\
\text { Mean } \\
\text { Diameter }\end{array}$ \\
\hline $0.30-0.49$ & .38 & 0.21 & 32947 & \multirow{6}{*}{1.45} \\
\hline $0.50-0.99$ & .70 & 0.30 & 25964 & \\
\hline $0.00-1.99$ & 1.41 & 0.30 & 13199 & \\
\hline $2.00-4.99$ & 3.16 & 0.40 & 18745 & \\
\hline $5.00-9.99$ & 7.07 & 0.30 & 2165 & \\
\hline$>10.00$ & 14.14 & 0.30 & 867 & \\
\hline \multicolumn{5}{|c|}{ Sample \#10 } \\
\hline \begin{tabular}{l|} 
Bin \\
Range \\
$(\mathrm{mm})$ \\
\end{tabular} & $\begin{array}{l}\text { Bin Mean } \\
\text { Diameter } \\
(\mu \mathrm{m})\end{array}$ & $\begin{array}{l}\text { Diff. of the } \\
\text { log of the } \\
\text { Size }\end{array}$ & Counts & $\begin{array}{l}\text { Weighted } \\
\text { Mean } \\
\text { Diameter }\end{array}$ \\
\hline $0.30-0.49$ & .38 & 0.21 & 26038 & \multirow{6}{*}{1.87} \\
\hline $0.50-0.99$ & .70 & 0.30 & 30092 & \\
\hline $1.00-1.99$ & 1.41 & 0.30 & 18762 & \\
\hline $2.00-4.99$ & 3.16 & 0.40 & 32145 & \\
\hline $5.00-9.99$ & 7.07 & 0.30 & 4320 & \\
\hline$>10.00$ & 14.14 & 0.30 & 1527 & \\
\hline
\end{tabular}


Sample \#11

\begin{tabular}{||l|l|l|r|r||}
\hline $\begin{array}{l}\text { Bin } \\
\text { Range } \\
(\mathrm{m})\end{array}$ & $\begin{array}{l}\text { Bin Mean } \\
\text { Diameter } \\
(\mu \mathrm{m})\end{array}$ & $\begin{array}{l}\text { Diff. of the } \\
\text { log of the } \\
\text { Size }\end{array}$ & Counts & $\begin{array}{l}\text { Weighted } \\
\text { Mean } \\
\text { Diameter }\end{array}$ \\
\hline $0.30-0.49$ & 0.38 & 0.21 & 26950 & \\
\hline $0.50-0.99$ & 0.70 & 0.30 & 28139 & \multirow{2}{*}{1.79} \\
\hline $1.00-1.99$ & 1.41 & 0.30 & 17128 & \\
\hline $2.00-4.99$ & 3.16 & 0.40 & 29193 & \\
\hline $5.00-9.99$ & 7.07 & 0.30 & 4032 & \\
\hline$>10.00$ & 14.14 & 0.30 & 1114 & \\
\hline
\end{tabular}

Samole \#12

\begin{tabular}{|c|c|c|c|c|}
\hline $\begin{array}{l}\text { Bin } \\
\text { Range } \\
(u m)\end{array}$ & \begin{tabular}{|l} 
Bin Mean \\
Diameter \\
$(\mathrm{um})$
\end{tabular} & $\begin{array}{l}\text { Diff. of the } \\
\text { log of the } \\
\text { Size }\end{array}$ & Counts & $\begin{array}{l}\text { Weighted } \\
\text { Mean } \\
\text { Diameter }\end{array}$ \\
\hline $0.30-0.49$ & .38 & 0.21 & 26872 & \multirow{6}{*}{1.76} \\
\hline $0.50-0.99$ & .70 & 0.30 & 27553 & \\
\hline $1.00-1.99$ & 1.41 & 0.30 & 16551 & \\
\hline $2.00-4.99$ & 3.16 & 0.40 & 27966 & \\
\hline $5.00-9.99$ & 7.07 & 0.30 & 3676 & \\
\hline$>10.00$ & 14.14 & 0.30 & 1096 & \\
\hline
\end{tabular}




\section{Aerosol Particle Measurement Record}

Description of sample location 3706 A Building, Resuspension Test, at $2.0 \mathrm{~m}$, Date 8/10/94, Time sample started 13:03:12, Temperature 80.5 F, Humidity $36.3 \%$, Dew Point 56.8 F, Average Wind Velocity (if applicable) $.00 \mathrm{~m} / \mathrm{s}$, \# of physical barriers between sample area and outside (if applicable) 1

Sample \#1

\begin{tabular}{||l|c|c|r|r|}
\hline $\begin{array}{l}\text { Bin } \\
\text { Range } \\
(\mu \mathrm{m})\end{array}$ & $\begin{array}{l}\text { Bin Mean } \\
\text { Diameter } \\
(\mu \mathrm{m})\end{array}$ & $\begin{array}{l}\text { Diff. of the } \\
\text { log of the } \\
\text { Size }\end{array}$ & Counts & $\begin{array}{l}\text { Weighted } \\
\text { Mean } \\
\text { Diameter }\end{array}$ \\
\hline $0.30-0.49$ & 0.38 & 0.21 & 37482 & \\
\hline $0.50-0.99$ & 0.70 & 0.30 & 31023 & \multirow{2}{*}{0.74} \\
\hline $1.00-1.99$ & 1.41 & 0.30 & 4547 & \\
\hline $2.00-4.99$ & 3.16 & 0.40 & 3950 & \\
\hline $5.00-9.99$ & 7.07 & 0.30 & 160 & \\
\hline$>10.00$ & 14.14 & 0.30 & 87 & \\
\hline
\end{tabular}

Sample $\# 3$

\begin{tabular}{|c|c|c|c|c|}
\hline $\begin{array}{l}\text { Bin } \\
\text { Range } \\
\text { (um) }\end{array}$ & \begin{tabular}{|l} 
Bin Mean \\
Diameter \\
$(\mu \mathrm{m})$
\end{tabular} & $\begin{array}{l}\text { Diff. of the } \\
\text { log of the } \\
\text { Size }\end{array}$ & Counts & $\begin{array}{l}\text { Weighted } \\
\text { Mean } \\
\text { Diameter }\end{array}$ \\
\hline $0.30-0.49$ & 0.38 & 0.21 & 32646 & \multirow{6}{*}{1.16} \\
\hline $0.50-0.99$ & 0.70 & 0.30 & 42633 & \\
\hline $1.00-1.99$ & 1.41 & 0.30 & 10516 & \\
\hline $2.00-4.99$ & 3.16 & 0.40 & 11243 & \\
\hline $5.00-9.99$ & 7.07 & 0.30 & 1168 & \\
\hline$>10.00$ & 14.14 & 0.30 & 1024 & \\
\hline \multicolumn{5}{|l|}{ Sample \#5 } \\
\hline $\begin{array}{l}\text { Bin } \\
\text { Range } \\
\text { (urm) } \\
\end{array}$ & $\begin{array}{l}\text { Bin Mean } \\
\text { Diameter } \\
(\mu \mathrm{m})\end{array}$ & $\begin{array}{l}\text { Diff. of the } \\
\text { log of the } \\
\text { Size }\end{array}$ & Counts & $\begin{array}{l}\text { Weighted } \\
\text { Mean } \\
\text { Diameter }\end{array}$ \\
\hline $0.30-0.49$ & 0.38 & 0.21 & 25497 & \multirow{6}{*}{1.54} \\
\hline 0.50 .0 .99 & 0.70 & 0.30 & 67151 & \\
\hline $1.00-1.99$ & 1.41 & 0.30 & 22073 & \\
\hline $2.00 \cdot 4.99$ & 3.16 & 0.40 & 24483 & \\
\hline $5.00-9.99$ & 7.07 & 0.30 & 2363 & \\
\hline$>10.00$ & 14.14 & 0.30 & 2901 & \\
\hline
\end{tabular}

\begin{tabular}{||l|c|c|r|r||}
\hline \begin{tabular}{|l|l|r||} 
Rin \\
Range
\end{tabular} & $\begin{array}{l}\text { Bin Mean } \\
\text { Diameter } \\
(\mu \mathrm{m})\end{array}$ & $\begin{array}{l}\text { Diff. of the } \\
\text { log of the } \\
\text { Size }\end{array}$ & Counts & $\begin{array}{l}\text { Weighted } \\
\text { Mean } \\
\text { Diameter }\end{array}$ \\
\hline $0.30-0.49$ & 0.38 & 0.21 & 21112 & \\
\hline $0.50-0.99$ & 0.70 & 0.30 & 75909 & \multirow{2}{*}{1.80} \\
\hline $1.00-1.99$ & 1.41 & 0.30 & 30434 & \\
\hline $2.00-4.99$ & 3.16 & 0.40 & 36509 & \\
\hline $5.00-9.99$ & 7.07 & 0.30 & 3916 & \\
\hline$>10.00$ & 14.14 & 0.30 & 4454 & \\
\hline
\end{tabular}

\begin{tabular}{|c|c|c|c|c|}
\hline \multicolumn{5}{|l|}{ Sample \#9 } \\
\hline $\begin{array}{l}\text { Bin } \\
\text { Range } \\
(\mu \mathrm{m})\end{array}$ & \begin{tabular}{|l|} 
Bin Mean \\
Diameter \\
$(\mu \mathrm{m})$
\end{tabular} & $\begin{array}{l}\text { Diff. of the } \\
\log \text { of the } \\
\text { Size }\end{array}$ & Counts & \begin{tabular}{|l|} 
Weighted \\
Mean \\
Diameter
\end{tabular} \\
\hline $0.30-0.49$ & 0.38 & 0.21 & 18747 & \\
\hline $0.50-0.99$ & 0.70 & 0.30 & 77685 & \\
\hline $1.00-1.99$ & 1.41 & 0.30 & 35743 & \\
\hline $2.00-4.99$ & 3.16 & 0.40 & 45840 & \\
\hline $5.00-9.99$ & 7.07 & 0.30 & 5310 & \\
\hline$>10.00$ & 14.14 & 0.30 & 6144 & \\
\hline
\end{tabular}

Sample \#2

\begin{tabular}{||c|c|c|r|r||}
\hline $\begin{array}{l}\text { Bin } \\
\text { Range } \\
(\mu \mathrm{m})\end{array}$ & $\begin{array}{l}\text { Bin Mean } \\
\text { Diameter } \\
(\mathrm{mm})\end{array}$ & $\begin{array}{l}\text { Diff. of the } \\
\text { log of the } \\
\text { Size }\end{array}$ & Counts & $\begin{array}{l}\text { Weighted } \\
\text { Mean } \\
\text { Diameter }\end{array}$ \\
\hline $0.30-0.49$ & .38 & 0.21 & 36468 & \\
\hline $0.50-0.99$ & .70 & 0.30 & 31863 & \multirow{2}{*}{0.80} \\
\hline $1.00-1.99$ & 1.41 & 0.30 & 5095 & \\
\hline $2.00-4.99$ & 3.16 & 0.40 & 4609 & \\
\hline $5.00-9.99$ & 7.07 & 0.30 & 298 & \\
\hline$>10.00$ & 14.14 & 0.30 & 193 & \\
\hline
\end{tabular}

\begin{tabular}{|c|c|c|c|c|}
\hline \multicolumn{5}{|l|}{ Sample \#4 } \\
\hline $\begin{array}{l}\text { Bin } \\
\text { Range } \\
(\mu \mathrm{m})\end{array}$ & \begin{tabular}{|l} 
Bin Mean \\
Diameter \\
$($ (um)
\end{tabular} & $\begin{array}{l}\text { Diff. of the } \\
\text { log of the } \\
\text { Size }\end{array}$ & Counts & $\begin{array}{l}\text { Weighted } \\
\text { Mean } \\
\text { Diameter }\end{array}$ \\
\hline $0.30-0.49$ & .38 & 0.21 & 23602 & \multirow{6}{*}{1.67} \\
\hline $0.50-0.99$ & .70 & 0.30 & 70717 & \\
\hline $1.00-1.99$ & 1.41 & 0.30 & 30610 & \\
\hline $2.00-4.99$ & 3.16 & 0.40 & 38622 & \\
\hline $5.00-9.99$ & 7.07 & 0.30 & 3284 & \\
\hline$>10.00$ & 74.14 & 0.30 & 2574 & \\
\hline
\end{tabular}

\begin{tabular}{|c|c|c|c|c|}
\hline $\begin{array}{l}\text { Bin } \\
\text { Range } \\
(\mu \mathrm{m})\end{array}$ & \begin{tabular}{|l} 
Bin Mean \\
Diameter \\
$(\mathrm{um})$
\end{tabular} & $\begin{array}{l}\text { Diff. of the } \\
\text { log of the } \\
\text { Size }\end{array}$ & Counts & \begin{tabular}{|l} 
Weighted \\
Mean \\
Diameter
\end{tabular} \\
\hline $0.30-0.49$ & .38 & 0.21 & 24218 & \multirow{6}{*}{1.74} \\
\hline $0.50-0.99$ & .70 & 0.30 & 69710 & \\
\hline $1.00-1.99$ & 1.41 & 0.30 & 23836 & \\
\hline $2.00-4.99$ & 3.16 & 0.40 & 27537 & \\
\hline $5.00-9.99$ & 7.07 & 0.30 & 3230 & \\
\hline$>10.00$ & 14.14 & 0.30 & 4560 & \\
\hline
\end{tabular}

Sample \#8

\begin{tabular}{|c|c|c|c|c|}
\hline \begin{tabular}{|l|} 
Bin \\
Range \\
$(\mu \mathrm{m})$
\end{tabular} & \begin{tabular}{|l|} 
Bin Mean \\
Diameter \\
$(\mathrm{um})$
\end{tabular} & $\begin{array}{l}\text { Diff. of the } \\
\log \text { of the } \\
\text { Size }\end{array}$ & Counts & $\begin{array}{l}\text { Weighted } \\
\text { Mean } \\
\text { Diameter }\end{array}$ \\
\hline $0.30-0.49$ & .38 & 0.21 & 19300 & \multirow{6}{*}{1.96} \\
\hline $0.50-0.99$ & .70 & 0.30 & 78960 & \\
\hline $0.00-1.99$ & 1.41 & 0.30 & 35864 & \\
\hline $2.00-4.99$ & 3.16 & 0.40 & 45827 & \\
\hline $5.00-9.99$ & 7.07 & 0.30 & 5006 & \\
\hline$>10.00$ & 14.14 & 0.30 & 5741 & \\
\hline
\end{tabular}

\begin{tabular}{|c|c|c|c|c|}
\hline \multicolumn{5}{|c|}{ Sample \#10 } \\
\hline $\begin{array}{l}\text { Bin } \\
\text { Range } \\
(u m)\end{array}$ & $\begin{array}{l}\text { Bin Mean } \\
\text { Diameter } \\
(\text { (rm) }\end{array}$ & $\begin{array}{l}\text { Diff. of the } \\
\text { log of the } \\
\text { Size }\end{array}$ & Counts & $\begin{array}{l}\text { Weighted } \\
\text { Mean } \\
\text { Diamoter }\end{array}$ \\
\hline $0.30-0.49$ & .38 & 0.21 & 18593 & \multirow{6}{*}{1.95} \\
\hline $0.50-0.99$ & .70 & 0.30 & 81403 & \\
\hline $1.00-1.99$ & 1.41 & 0.30 & 38885 & \\
\hline $2.00-4.99$ & 3.16 & 0.40 & 50160 & \\
\hline $5.00-9.99$ & 7.07 & 0.30 & 5429 & \\
\hline$>10.00$ & 14.14 & 0.30 & 5184 & \\
\hline
\end{tabular}


Sample \#11

\begin{tabular}{||l|c|c|r|r||}
\hline $\begin{array}{l}\text { Bin } \\
\text { Range } \\
(\mu \mathrm{m})\end{array}$ & $\begin{array}{l}\text { Bin Mean } \\
\text { Diameter } \\
(\mu \mathrm{m})\end{array}$ & $\begin{array}{l}\text { Diff. of the } \\
\text { log of the } \\
\text { Size }\end{array}$ & Counts & $\begin{array}{l}\text { Weighted } \\
\text { Mean } \\
\text { Diameter }\end{array}$ \\
\hline $0.30-0.49$ & 0.38 & 0.21 & 18926 & \\
\cline { 1 - 4 } $0.50-0.99$ & 0.70 & 0.30 & 80830 & \multirow{2}{*}{1.91} \\
\hline $1.00-1.99$ & 1.41 & 0.30 & 37636 & \\
\cline { 1 - 4 } $2.00-4.99$ & 3.16 & 0.40 & 48801 & \\
\hline $5.00-9.99$ & 7.07 & 0.30 & 4965 & \\
\cline { 1 - 4 }$>10.00$ & 14.14 & 0.30 & 4745 & \\
\hline
\end{tabular}

Sample $\# 13$

\begin{tabular}{||c|c|c|r|r||}
\hline $\begin{array}{l}\text { Bin } \\
\text { Range } \\
(\mu \mathrm{m})\end{array}$ & $\begin{array}{l}\text { Bin Mean } \\
\text { Diameter } \\
(\mu \mathrm{m})\end{array}$ & $\begin{array}{l}\text { Diff. of the } \\
\text { log of the } \\
\text { Size }\end{array}$ & Counts & $\begin{array}{l}\text { Weighted } \\
\text { Mean } \\
\text { Diameter }\end{array}$ \\
\hline $0.30 \cdot 0.49$ & 0.38 & 0.21 & 19082 & \\
\hline $0.50-0.99$ & 0.70 & 0.30 & 83207 & \multirow{2}{*}{1.84} \\
\hline $1.00-1.99$ & 1.41 & 0.30 & 40317 & \\
\hline $2.00-4.99$ & 3.16 & 0.40 & 51284 & \\
\hline $5.00-9.99$ & 7.07 & 0.30 & 4841 & \\
\hline$>10.00$ & 14.14 & 0.30 & 3899 & \\
\hline
\end{tabular}

Samole \#12

\begin{tabular}{|c|c|c|c|c|}
\hline $\begin{array}{l}\text { Bin } \\
\text { Range } \\
(\mu \mathrm{m})\end{array}$ & $\mid \begin{array}{l}\text { Bin Mean } \\
\text { Diameter } \\
((\mathrm{rm})\end{array}$ & $\begin{array}{l}\text { Diff. of the } \\
\log \text { of the } \\
\text { Size }\end{array}$ & Counts & $\begin{array}{l}\text { Weighted } \\
\text { Mean } \\
\text { Diameter }\end{array}$ \\
\hline $0.30-0.49$ & .38 & 0.21 & 19584 & \multirow{6}{*}{1.84} \\
\hline $0.50-0.99$ & .70 & 0.30 & 80913 & \\
\hline $1.00-1.99$ & 1.41 & 0.30 & 38139 & \\
\hline $2.00-4.99$ & 3.16 & 0.40 & 48307 & \\
\hline $5.00-9.99$ & 7.07 & 0.30 & 4683 & \\
\hline$>10.00$ & 14.14 & 0.30 & 3965 & \\
\hline
\end{tabular}

$$
\text { Sample \#14 }
$$

\begin{tabular}{|c|c|c|c|c|}
\hline $\begin{array}{l}\text { Bin } \\
\text { Range } \\
\text { ( } m \text { ) }\end{array}$ & \begin{tabular}{|l} 
Bin Mean \\
Diameter \\
$(\mu \mathrm{m})$
\end{tabular} & $\begin{array}{l}\text { Diff. of the } \\
\text { log of the } \\
\text { Size }\end{array}$ & Counts & $\begin{array}{l}\text { Weighted } \\
\text { Mean } \\
\text { Diameter }\end{array}$ \\
\hline $0.30-0.49$ & .38 & 0.21 & 19542 & \multirow{6}{*}{1.78} \\
\hline $0.50-0.99$ & .70 & 0.30 & 81177 & \\
\hline $1.00-1.99$ & 1.41 & 0.30 & 37907 & \\
\hline $2.00-4.99$ & 3.16 & 0.40 & 48398 & \\
\hline $5.00-9.99$ & 7.07 & 0.30 & 4488 & \\
\hline$>10.00$ & 14.14 & 0.30 & 3121 & \\
\hline
\end{tabular}




\section{Aerosol Particle Measurement Record}

Description of sample location 3706A Building, Resuspension Test, at .25 m, Date 8/10/94, Time sample started 13:03:12, Temperature80.5 F, Humidity 36.3 \%, Dew Point $56.8 \mathrm{~F}$, Average Wind Velocity (if applicable) $.00 \mathrm{~m} / \mathrm{s}$, \# of physical barriers between sample area and outside (if applicable) 1

\begin{tabular}{|c|c|c|c|c|}
\hline $\begin{array}{l}\text { Bin } \\
\text { Range } \\
(\omega m)\end{array}$ & \begin{tabular}{|l|} 
Bin Mean \\
Diameter \\
$(u m)$
\end{tabular} & $\begin{array}{l}\text { Diff. of the } \\
\log \text { of the } \\
\text { Size }\end{array}$ & Counts & $\begin{array}{l}\text { Weighted } \\
\text { Mean } \\
\text { Diameter }\end{array}$ \\
\hline $0.30-0.49$ & 0.38 & 0.21 & 46299 & \multirow{6}{*}{0.83} \\
\hline $0.50-0.99$ & 0.70 & 0.30 & 17838 & \\
\hline $1.00-1.99$ & 1.41 & 0.30 & 5356 & \\
\hline $2.00-4.99$ & 3.16 & 0.40 & 6090 & \\
\hline $5.00-9.99$ & 7.07 & 0.30 & 570 & \\
\hline$>10.00$ & 14.14 & 0.30 & 177 & \\
\hline \multicolumn{5}{|l|}{ Sample \#3 } \\
\hline $\begin{array}{l}\text { Bin } \\
\text { Range } \\
(\mu \mathrm{m})\end{array}$ & \begin{tabular}{|l} 
Bin Mean \\
Diameter \\
$($ (um)
\end{tabular} & $\begin{array}{l}\text { Diff. of the } \\
\log \text { of the } \\
\text { Size }\end{array}$ & Counts & \begin{tabular}{|l|} 
Weighted \\
Mean \\
Diameter
\end{tabular} \\
\hline $0.30-0.49$ & 0.38 & 0.21 & 4376 & \multirow{6}{*}{6.84} \\
\hline $0.50-0.99$ & 0.70 & 0.30 & 12941 & \\
\hline $1.00-1.99$ & 1.41 & 0.30 & 17087 & \\
\hline $2.00-4.99$ & 3.16 & 0.40 & 99838 & \\
\hline $5.00-9.99$ & 7.07 & 0.30 & 56300 & \\
\hline$>10.00$ & 14.14 & 0.30 & 76029 & \\
\hline \multicolumn{5}{|l|}{ Sample \#5 } \\
\hline $\begin{array}{l}\text { Bin } \\
\text { Range } \\
(\mu \mathrm{m})\end{array}$ & \begin{tabular}{|l}
$\begin{array}{l}\text { Bin Mean } \\
\text { Diameter } \\
(\mu \mathrm{m})\end{array}$ \\
\end{tabular} & \begin{tabular}{|l|} 
Diff. of the \\
log of the \\
Size
\end{tabular} & Counts & $\begin{array}{l}\text { Weighted } \\
\text { Mean } \\
\text { Diameter }\end{array}$ \\
\hline $0.30 \cdot 0.49$ & 0.38 & 0.21 & 28428 & \multirow{6}{*}{1.94} \\
\hline $0.50 \cdot 0.99$ & 0.70 & 0.30 & 29524 & \\
\hline $1.00-1.99$ & 1.41 & 0.30 & 18008 & \\
\hline $2,00-4.99$ & 3.16 & 0.40 & 30997 & \\
\hline $5.00-9.99$ & 7.07 & 0.30 & 4652 & \\
\hline$>10.00$ & 14.14 & 0.30 & 2379 & \\
\hline \multicolumn{5}{|l|}{ Sample \#7 } \\
\hline \begin{tabular}{|l|} 
Bin \\
Range \\
$(\mu \mathrm{m})$
\end{tabular} & \begin{tabular}{|l|}
$\begin{array}{l}\text { Bin Mean } \\
\text { Diameter } \\
(\mu \mathrm{m})\end{array}$ \\
\end{tabular} & \begin{tabular}{|l|} 
Diff. of the \\
log of the \\
Size
\end{tabular} & Counts & \begin{tabular}{|l|} 
Weighted \\
Mean \\
Diameter
\end{tabular} \\
\hline $0.30-0.49$ & 0.38 & 0.21 & 24414 & \multirow{6}{*}{2.13} \\
\hline $0.50-0.99$ & 0.70 & 0.30 & 33692 & \\
\hline $1.00-1.99$ & 1.41 & 0.30 & 22693 & \\
\hline $2.00-4.99$ & 3.16 & 0.40 & 40659 & \\
\hline $5.00-9.99$ & 7.07 & 0.30 & 6080 & \\
\hline$>10.00$ & 14.14 & 0.30 & 2898 & \\
\hline \multicolumn{5}{|l|}{ Sample \#9 } \\
\hline $\begin{array}{l}\text { Bin } \\
\text { Range } \\
(\mu \mathrm{m})\end{array}$ & \begin{tabular}{|l|} 
Bin Mean \\
Diameter \\
$(u m)$
\end{tabular} & \begin{tabular}{|l|} 
Diff. of the \\
log of the \\
Size
\end{tabular} & Counts & \begin{tabular}{|l|} 
Weighted \\
Mean \\
Diameter
\end{tabular} \\
\hline $0.30-0.49$ & 0.38 & 0.21 & 20706 & \multirow{6}{*}{2.39} \\
\hline $0.50-0.99$ & 0.70 & 0.30 & 37622 & \\
\hline $1.00-1.99$ & 1.41 & 0.30 & 30476 & \\
\hline $2.00-4.99$ & 3.16 & 0.40 & 61317 & \\
\hline $5.00-9.99$ & 7.07 & 0.30 & 9677 & \\
\hline$>10.00$ & 14.14 & 0.30 & 3554 & \\
\hline
\end{tabular}

\begin{tabular}{|c|c|c|c|c|}
\hline $\begin{array}{l}\text { Bin } \\
\text { Range } \\
(u m)\end{array}$ & \begin{tabular}{|l} 
Bin Mean \\
Diameter \\
$(\mathrm{(mm})$
\end{tabular} & \begin{tabular}{|l|} 
Diff. of the \\
$\log$ of the \\
Size
\end{tabular} & Counts & \begin{tabular}{|l|} 
Weighted \\
Mean \\
Diameter
\end{tabular} \\
\hline $0.30-0.49$ & .38 & 0.21 & 931 & \\
\hline $0.50-0.99$ & .70 & 0.30 & 2896 & \\
\hline $1.00-1.99$ & 1.41 & 0.30 & 3682 & \\
\hline $2.00-4.99$ & 3.16 & 0.40 & 32216 & \\
\hline $5.00-9.99$ & 7.07 & 0.30 & 30361 & \\
\hline$>10.00$ & 14.14 & 0.30 & 194704 & \\
\hline \multicolumn{5}{|l|}{ Sample \#4 } \\
\hline \begin{tabular}{|l|} 
Bin \\
Range \\
(um)
\end{tabular} & \begin{tabular}{|l} 
Bin Mean \\
Diameter \\
(um)
\end{tabular} & $\begin{array}{l}\text { Diff. of the } \\
\text { log of the } \\
\text { Size }\end{array}$ & Counts & $\begin{array}{l}\text { Weighted } \\
\text { Mean } \\
\text { Diameter }\end{array}$ \\
\hline $0.30-0.49$ & .38 & 0.21 & 14259 & \multirow{6}{*}{3.38} \\
\hline $0.50-0.99$ & .70 & 0.30 & 33529 & \\
\hline $1.00-1.99$ & 1.41 & 0.30 & 34274 & \\
\hline $2.00-4.99$ & 3.16 & 0.40 & 100322 & \\
\hline $5.00-9.99$ & 7.07 & 0.30 & 22792 & \\
\hline$>10.00$ & 14.14 & 0.30 & 12864 & \\
\hline \multicolumn{5}{|c|}{ Sample \#6 } \\
\hline $\begin{array}{l}\text { Bin } \\
\text { Range } \\
(\omega \mathrm{m}) \\
\end{array}$ & \begin{tabular}{|l} 
Bin Mean \\
Diameter \\
$(u m)$
\end{tabular} & $\begin{array}{l}\text { Diff. of the } \\
\text { log of the } \\
\text { Size }\end{array}$ & Counts & \begin{tabular}{|l|} 
Weighted \\
Mean \\
Diameter
\end{tabular} \\
\hline $0.30-0.49$ & .38 & 0.21 & 31459 & \multirow{6}{*}{1.70} \\
\hline $0.50-0.99$ & .70 & 0.30 & 27479 & \\
\hline $1.00-1.99$ & 1.41 & 0.30 & 15131 & \\
\hline $2.00-4.99$ & 3.16 & 0.40 & 23544 & \\
\hline $5.00-9.99$ & 7.07 & 0.30 & 3362 & \\
\hline$>10.00$ & 14.14 & 0.30 & 1659 & \\
\hline \multicolumn{5}{|c|}{ Sample \#8 } \\
\hline $\begin{array}{l}\text { Bin } \\
\text { Range } \\
(\mu \mathrm{m}) \\
\end{array}$ & $\begin{array}{l}\text { Bin Mean } \\
\text { Diameter } \\
(\mu \mathrm{m})\end{array}$ & $\begin{array}{l}\text { Diff. of the } \\
\text { log of the } \\
\text { Size }\end{array}$ & Counts & $\begin{array}{l}\text { Weighted } \\
\text { Mean } \\
\text { Diamoter }\end{array}$ \\
\hline $0.30-0.49$ & \begin{tabular}{|r}
.38 \\
\end{tabular} & 0.21 & 23146 & \multirow{6}{*}{2.19} \\
\hline $0.50-0.99$ & .70 & 0.30 & 35430 & \\
\hline $0.00-1.99$ & 1.41 & 0.30 & 25159 & \\
\hline $2.00-4.99$ & 3.16 & 0.40 & 45407 & \\
\hline $5.00-9.99$ & 7.07 & 0.30 & 6817 & \\
\hline$>10.00$ & 14.14 & 0.30 & 3093 & \\
\hline \multicolumn{5}{|c|}{ Sample $\# 10$} \\
\hline $\begin{array}{l}\text { Bin } \\
\text { Range } \\
(\mu \mathrm{m})\end{array}$ & $\begin{array}{l}\text { Bin Mean } \\
\text { Diameter } \\
(\mu \mathrm{m})\end{array}$ & \begin{tabular}{|l|} 
Diff. of the \\
log of the \\
Size
\end{tabular} & Counts & \begin{tabular}{|l|} 
Weighted \\
Mean \\
Diameter
\end{tabular} \\
\hline $0.30-0.49$ & .38 & 0.21 & 19227 & \multirow{6}{*}{2.50} \\
\hline $0.50-0.99$ & .70 & 0.30 & 38843 & \\
\hline $1.00-1.99$ & 1.41 & 0.30 & 33543 & \\
\hline $2.00-4.99$ & 3.16 & 0.40 & 72983 & \\
\hline $5.00-9.99$ & 7.07 & 0.30 & 11864 & \\
\hline$>10.00$ & 14.14 & 0.30 & 3898 & \\
\hline
\end{tabular}


WHC-MR-0496

\begin{tabular}{|c|c|c|c|c|}
\hline $\begin{array}{l}\text { Bin } \\
\text { Range } \\
(\mu \mathrm{m})\end{array}$ & $\begin{array}{l}\text { Bin Mean } \\
\text { Diameter } \\
\text { (um) }\end{array}$ & $\begin{array}{l}\text { Diff. of the } \\
\text { log of the. } \\
\text { Size }\end{array}$ & Counts & $\begin{array}{l}\text { Weighted } \\
\text { Mean } \\
\text { Diameter }\end{array}$ \\
\hline $0.30-0.49$ & 0.38 & 0.21 & 18256 & \multirow{6}{*}{2.57} \\
\hline $0.50-0.99$ & 0.70 & 0.30 & 39214 & \\
\hline $1.00-1.99$ & 1.41 & 0.30 & 35010 & \\
\hline $2.00-4.99$ & 3.16 & 0.40 & 80158 & \\
\hline $5.00 \cdot 9.99$ & 7.07 & 0.30 & 13383 & \\
\hline$>10.00$ & 14.14 & 0.30 & 4011 & \\
\hline
\end{tabular}

Sample \#12

\begin{tabular}{|c|c|c|c|c|}
\hline $\begin{array}{l}\text { Bin } \\
\text { Range } \\
\text { (um) }\end{array}$ & $\begin{array}{l}\text { Bin Mean } \\
\text { Diameter } \\
(\mu \mathrm{m})\end{array}$ & $\begin{array}{l}\text { Diff. of the } \\
\text { log of the } \\
\text { Size }\end{array}$ & Counts & $\begin{array}{l}\text { Weighted } \\
\text { Mean } \\
\text { Diameter }\end{array}$ \\
\hline $0.30-0.49$ & .38 & 0.21 & 18368 & \multirow{6}{*}{2.55} \\
\hline $0.50-0.99$ & .70 & 0.30 & 38589 & \\
\hline $1.00-1.99$ & 1.41 & 0.30 & 34902 & \\
\hline $2.00-4.99$ & 3.16 & 0.40 & 78684 & \\
\hline $5.00-9.99$ & 7.07 & 0.30 & 12949 & \\
\hline$>10.00$ & 14.14 & 0.30 & 3795 & \\
\hline
\end{tabular}

\begin{tabular}{|c|c|c|c|c|}
\hline $\begin{array}{l}\text { Bin } \\
\text { Range } \\
(u m)\end{array}$ & $\begin{array}{l}\text { Bin Mean } \\
\text { Diameter } \\
(e r m)\end{array}$ & $\begin{array}{l}\text { Diff. of the } \\
\text { log of the } \\
\text { Size }\end{array}$ & Counts & $\begin{array}{l}\text { Weighted } \\
\text { Mean } \\
\text { Diameter }\end{array}$ \\
\hline $0.30-0.49$ & 0.38 & 0.21 & 19529 & \multirow{6}{*}{2.47} \\
\hline $0.50-0.99$ & 0.70 & 0.30 & 38701 & \\
\hline $1.00-1.99$ & 1.41 & 0.30 & 33410 & \\
\hline $2,00-4.99$ & 3.16 & 0.40 & 73292 & \\
\hline $5.00 \cdot 9.99$ & 7.07 & 0.30 & 11939 & \\
\hline$>10.00$ & 14.14 & 0.30 & 3313 & \\
\hline
\end{tabular}

\begin{tabular}{|c|c|c|c|c|}
\hline $\begin{array}{l}\text { Bin } \\
\text { Range } \\
(\mu \mathrm{m})\end{array}$ & $\begin{array}{l}\text { Bin Mean } \\
\text { Diameter } \\
(\mu \mathrm{m})\end{array}$ & $\begin{array}{l}\text { Diff. of the } \\
\text { log of the } \\
\text { Size }\end{array}$ & Counts & $\begin{array}{l}\text { Weighted } \\
\text { Mean } \\
\text { Diameter }\end{array}$ \\
\hline $0.30-0.49$ & .38 & 0.21 & 20512 & \multirow{6}{*}{2.36} \\
\hline $0.50-0.99$ & .70 & 0.30 & 38809 & \\
\hline $1.00-1.99$ & 1.41 & 0.30 & 31778 & \\
\hline $2.00-4.99$ & 3.16 & 0.40 & 66830 & \\
\hline $5.00-9.99$ & 7.07 & 0.30 & 10368 & \\
\hline$>10.00$ & 14.14 & 0.30 & 2769 & \\
\hline
\end{tabular}




\section{Aerosol Particle Measurement Record}

Description of sample location 3706A Building, Resuspension Test, at $2.0 \mathrm{~m}$, Date 8/11/94, Time sample started 7:47:09, Temperature $77.8 \mathrm{~F}$, Humidity $41.3 \%$, Dew Point $52.7 \mathrm{~F}$, Average Wind Velocity (if applicable) $.00 \mathrm{~m} / \mathrm{s}$, \# of physical barriers between sample area and outside (if applicable) 1

Sample \#1

\begin{tabular}{|c|c|c|c|c|}
\hline $\begin{array}{l}\text { Bin } \\
\text { Range } \\
\text { (um) }\end{array}$ & $\begin{array}{l}\text { Bin Mean } \\
\text { Diameter } \\
(u m)\end{array}$ & $\begin{array}{l}\text { Diff. of the } \\
\log \text { of the } \\
\text { Size }\end{array}$ & Counts & $\begin{array}{l}\text { Weighted } \\
\text { Mean } \\
\text { Diameter }\end{array}$ \\
\hline $0.30-0.49$ & 0.38 & 0.21 & 46036 & \multirow{6}{*}{0.83} \\
\hline $0.50-0.99$ & 0.70 & 0.30 & 52816 & \\
\hline $1.00-1.99$ & 1.41 & 0.30 & 10036 & \\
\hline $2.00-4.99$ & 3.16 & 0.40 & 7829 & \\
\hline $5.00-9.99$ & 7.07 & 0.30 & 288 & \\
\hline$>10.00$ & 14.14 & 0.30 & 103 & \\
\hline \multicolumn{5}{|l|}{ Sample \#3 } \\
\hline \begin{tabular}{|l} 
Bin \\
Range \\
$(u m)$
\end{tabular} & $\begin{array}{l}\text { Bin Mean } \\
\text { Diameter } \\
(u m)\end{array}$ & $\begin{array}{l}\text { Diff. of the } \\
\text { log of the } \\
\text { Size }\end{array}$ & Counts & $\begin{array}{l}\text { Weighted } \\
\text { Mean } \\
\text { Diameter }\end{array}$ \\
\hline $0.30-0.49$ & 0.38 & 0.21 & 43440 & \multirow{6}{*}{0.88} \\
\hline $0.50-0.99$ & 0.70 & 0.30 & 55026 & \\
\hline $1.00-1.99$ & 1.41 & 0.30 & 11257 & \\
\hline $2,00-4.99$ & 3.16 & 0.40 & 8911 & \\
\hline $5.00-9.99$ & 7.07 & 0.30 & 413 & \\
\hline$>10.00$ & 14.14 & 0.30 & 176 & \\
\hline \multicolumn{5}{|l|}{ Sample \#5 } \\
\hline \begin{tabular}{|l} 
Bin \\
Range \\
$(\mu \mathrm{m})$
\end{tabular} & $\begin{array}{l}\text { Bin Mean } \\
\text { Diameter } \\
(\text { (rm) }\end{array}$ & $\begin{array}{l}\text { Diff. of the } \\
\log \text { of the } \\
\text { Size }\end{array}$ & Counts & $\begin{array}{l}\text { Weighted } \\
\text { Mean } \\
\text { Diameter }\end{array}$ \\
\hline $0.30-0.49$ & 0.38 & 0.21 & 16932 & \\
\hline $0.50 \cdot 0.99$ & 0.70 & 0.30 & 83904 & \\
\hline $1.00-1.99$ & 1.41 & 0.30 & 46648 & \\
\hline $2.00-4.99$ & 3.16 & 0.40 & 68800 & \\
\hline $5.00 \cdot 9.99$ & 7.07 & 0.30 & 7595 & \\
\hline$>10.00$ & 14.14 & 0.30 & 6232 & \\
\hline \multicolumn{5}{|l|}{ Sample \#7 } \\
\hline $\begin{array}{l}\text { Bin } \\
\text { Range } \\
(\mu \mathrm{m})\end{array}$ & $\begin{array}{l}\text { Bin Mean } \\
\text { Diameter } \\
(\mu \mathrm{m})\end{array}$ & $\begin{array}{l}\text { Diff. of the } \\
\log \text { of the } \\
\text { Size }\end{array}$ & Counts & $\begin{array}{l}\text { Weighted } \\
\text { Mean } \\
\text { Diameter }\end{array}$ \\
\hline $0.30-0.49$ & 0.38 & 0.21 & 19344 & \multirow{6}{*}{1.90} \\
\hline $0.50-0.99$ & 0.70 & 0.30 & 84824 & \\
\hline $1.00-1.99$ & 1.41 & 0.30 & 41330 & \\
\hline $2.00-4.99$ & 3.16 & 0.40 & 55947 & \\
\hline $5.00-9.99$ & 7.07 & 0.30 & 5705 & \\
\hline$>10.00$ & 14.14 & 0.30 & 4246 & \\
\hline \multicolumn{5}{|l|}{ Sample \#9 } \\
\hline $\begin{array}{l}\text { Bin } \\
\text { Range } \\
(u \mathrm{~m}) \\
\end{array}$ & $\begin{array}{l}\text { Bin Mean } \\
\text { Diameter } \\
(u m)\end{array}$ & $\begin{array}{l}\text { Diff. of the } \\
\text { log of the } \\
\text { Size }\end{array}$ & Counts & $\begin{array}{l}\text { Weighted } \\
\text { Mean } \\
\text { Diameter }\end{array}$ \\
\hline $0.30-0.49$ & 0.38 & 0.21 & 22048 & \multirow{6}{*}{1.71} \\
\hline $0.50-0.99$ & 0.70 & 0.30 & 83498 & \\
\hline $1.00-1.99$ & 1.41 & 0.30 & 35626 & \\
\hline $2.00-4.99$ & 3.16 & 0.40 & 43528 & \\
\hline $5.00-9.99$ & 7.07 & 0.30 & 4199 & \\
\hline$>10.00$ & 14.14 & 0.30 & 3076 & \\
\hline
\end{tabular}

Sample \#2

\begin{tabular}{|c|c|c|c|c|}
\hline $\begin{array}{l}\text { Bin } \\
\text { Range } \\
(\mu m)\end{array}$ & $\mid \begin{array}{l}\text { Bin Mean } \\
\text { Diameter } \\
(\mathrm{erm})\end{array}$ & $\begin{array}{l}\text { Diff. of the } \\
\log \text { of the } \\
\text { Size }\end{array}$ & Counts & $\begin{array}{l}\text { Weighted } \\
\text { Mean } \\
\text { Diameter }\end{array}$ \\
\hline $0.30-0.49$ & .38 & 0.21 & 45821 & \multirow{6}{*}{0.82} \\
\hline $0.50-0.99$ & .70 & 0.30 & 52985 & \\
\hline $1.00-1.99$ & 1.41 & 0.30 & 10032 & \\
\hline $2.00-4.99$ & 3.16 & 0.40 & 7593 & \\
\hline $5.00-9.99$ & 7.07 & 0.30 & 297 & \\
\hline$>10.00$ & 14.14 & 0.30 & 90 & \\
\hline
\end{tabular}

Sample \#4

\begin{tabular}{|c|c|c|c|c|}
\hline $\begin{array}{l}\text { Bin } \\
\text { Range } \\
\text { (um) }\end{array}$ & $\begin{array}{l}\text { Bin Mean } \\
\text { Diameter } \\
(\text { (um) }\end{array}$ & $\begin{array}{l}\text { Diff. of the } \\
\text { log of the } \\
\text { Size }\end{array}$ & Counts & $\begin{array}{l}\text { Weighted } \\
\text { Mean } \\
\text { Diameter }\end{array}$ \\
\hline $0.30-0.49$ & .38 & 0.21 & 37847 & \multirow{6}{*}{1.39} \\
\hline $0.50-0.99$ & .70 & 0.30 & 71009 & \\
\hline $1.00-1.99$ & 1.41 & 0.30 & 23607 & \\
\hline $2.00-4.99$ & 3.16 & 0.40 & 25599 & \\
\hline $5.00-9.99$ & 7.07 & 0.30 & 2124 & \\
\hline$>10.00$ & 14.14 & 0.30 & 1768 & \\
\hline
\end{tabular}

Sample \#6

\begin{tabular}{|c|c|c|c|c|}
\hline $\begin{array}{l}\text { Bin } \\
\text { Range } \\
(\omega \mathrm{m})\end{array}$ & \begin{tabular}{|l|} 
Bin Mean \\
Diameter \\
$(\mathrm{erm})$
\end{tabular} & $\begin{array}{l}\text { Diff. of the } \\
\log \text { of the } \\
\text { Size }\end{array}$ & Counts & $\begin{array}{l}\text { Woighted } \\
\text { Mean } \\
\text { Diameter }\end{array}$ \\
\hline $0.30-0.49$ & .38 & 0.21 & 20247 & \multirow{6}{*}{1.86} \\
\hline $0.50-0.99$ & .70 & 0.30 & 84628 & \\
\hline $1.00-1.99$ & 1.41 & 0.30 & 39648 & \\
\hline $2.00-4.99$ & 3.16 & 0.40 & 51733 & \\
\hline $5.00-9.99$ & 7.07 & 0.30 & 5295 & \\
\hline$>10.00$ & 14.14 & 0.30 & 4086 & \\
\hline
\end{tabular}

Sample \#8

\begin{tabular}{||c|c|c|r|r||}
\hline $\begin{array}{l}\text { Bin } \\
\text { Range } \\
(\mu \mathrm{m})\end{array}$ & $\begin{array}{l}\text { Bin Mean } \\
\text { Diameter } \\
(\mu \mathrm{m})\end{array}$ & $\begin{array}{l}\text { Diff. of the } \\
\text { log of the } \\
\text { Size }\end{array}$ & Counts & $\begin{array}{l}\text { Weighted } \\
\text { Mean } \\
\text { Diameter }\end{array}$ \\
\hline $0.30-0.49$ & .38 & 0.21 & 21109 & \\
\hline $0.50-0.99$ & .70 & 0.30 & 83936 \\
\cline { 1 - 4 } & \multirow{2}{*}{1.77} \\
\hline $2.00-1.99$ & 1.41 & 0.30 & 37268 & \\
\hline $5.00-9.99$ & 3.16 & 0.40 & 47643 & \\
\hline$>10.00$ & 7.07 & 0.30 & 4622 \\
\hline
\end{tabular}

\begin{tabular}{|c|c|c|c|c|}
\hline \multicolumn{5}{|c|}{ Sample $\# 10$} \\
\hline $\begin{array}{l}\text { Bin } \\
\text { Range } \\
(\mu \mathrm{m})\end{array}$ & \begin{tabular}{|l|} 
Bin Mean \\
Diameter \\
$((\mathrm{m})$
\end{tabular} & $\begin{array}{l}\text { Diff. of the } \\
\log \text { of the } \\
\text { Size }\end{array}$ & Counts & $\begin{array}{l}\text { Weighted } \\
\text { Mean } \\
\text { Diameter }\end{array}$ \\
\hline $0.30-0.49$ & .38 & 0.21 & 23624 & \multirow{6}{*}{1.61} \\
\hline $0.50-0.99$ & .70 & 0.30 & 80851 & \\
\hline $1.00-1.99$ & 1.41 & 0.30 & 31498 & \\
\hline $2.00-4.99$ & 3.16 & 0.40 & 37733 & \\
\hline $5.00-9.99$ & 7.07 & 0.30 & 3554 & \\
\hline$>10.00$ & 14.14 & 0.30 & 2541 & \\
\hline
\end{tabular}




\begin{tabular}{|c|c|c|c|c|}
\hline $\begin{array}{l}\text { Bin } \\
\text { Range } \\
(\mu \mathrm{m})\end{array}$ & $\begin{array}{l}\text { Bin Mean } \\
\text { Diameter } \\
(\mathrm{erm})\end{array}$ & $\begin{array}{l}\text { Diff. of the } \\
\text { log of the } \\
\text { Size }\end{array}$ & Counts & $\begin{array}{l}\text { Weighted } \\
\text { Mean } \\
\text { Diameter }\end{array}$ \\
\hline $0.30-0.49$ & 0.38 & 0.21 & 24118 & \multirow{6}{*}{1.58} \\
\hline $0.50-0.99$ & 0.70 & 0.30 & 79421 & \\
\hline $1.00-1.99$ & 1.41 & 0.30 & 31030 & \\
\hline $2.00-4.99$ & 3.16 & 0.40 & 36595 & \\
\hline $5.00-9.99$ & 7.07 & 0.30 & 3283 & \\
\hline$>10.00$ & 14.14 & 0.30 & 2191 & \\
\hline \multicolumn{5}{|l|}{ Sample \#13 } \\
\hline $\begin{array}{l}\text { Bin } \\
\text { Range } \\
(\mu \mathrm{m})\end{array}$ & $\begin{array}{l}\text { Bin Mean } \\
\text { Diameter } \\
(\mathrm{um})\end{array}$ & $\begin{array}{l}\text { Diff. of the } \\
\text { log of the } \\
\text { Size }\end{array}$ & Counts & $\begin{array}{l}\text { Weighted } \\
\text { Mean } \\
\text { Diameter }\end{array}$ \\
\hline $0.30-0.49$ & 0.38 & 0.21 & 26479 & \multirow{6}{*}{1.47} \\
\hline $0.50-0.99$ & 0.70 & 0.30 & 76072 & \\
\hline $1.00-1.99$ & 1.41 & 0.30 & 27381 & \\
\hline $2,00-4.99$ & 3.16 & 0.40 & 31389 & \\
\hline $5.00-9.99$ & 7.07 & 0.30 & 2630 & \\
\hline$>10.00$ & 14.14 & 0.30 & 1736 & \\
\hline
\end{tabular}

Samole \#12

\begin{tabular}{|c|c|c|c|c|}
\hline $\begin{array}{l}\text { Bin } \\
\text { Range } \\
\text { (erm) }\end{array}$ & $\begin{array}{l}\text { Bin Mean } \\
\text { Diameter } \\
(\mu \mathrm{m})\end{array}$ & $\begin{array}{l}\text { Diff. of the } \\
\log \text { of the } \\
\text { Size }\end{array}$ & Counts & $\begin{array}{l}\text { Weighted } \\
\text { Mean } \\
\text { Diameter }\end{array}$ \\
\hline $0.30-0.49$ & .38 & 0.21 & 25760 & \multirow{6}{*}{1.48} \\
\hline $0.50-0.99$ & .70 & 0.30 & 77047 & \\
\hline $1.00-1.99$ & 1.41 & 0.30 & 28069 & \\
\hline $2.00-4.99$ & 3.16 & 0.40 & 32197 & \\
\hline $5.00-9.99$ & 7.07 & 0.30 & 2779 & \\
\hline$>10.00$ & 14.14 & 0.30 & 1580 & \\
\hline
\end{tabular}

\begin{tabular}{|c|c|c|c|c|}
\hline \multicolumn{5}{|c|}{ Sample \#14 } \\
\hline $\begin{array}{l}\text { Bin } \\
\text { Range } \\
\text { (um) }\end{array}$ & $\begin{array}{l}\text { Bin Mean } \\
\text { Diameter } \\
(\mathrm{erm})\end{array}$ & $\begin{array}{l}\text { Diff. of the } \\
\text { log of the } \\
\text { Size }\end{array}$ & Counts & $\begin{array}{l}\text { Weighted } \\
\text { Mean } \\
\text { Diameter }\end{array}$ \\
\hline $0.30-0.49$ & .38 & 0.21 & 25465 & \multirow{6}{*}{1.49} \\
\hline $0.50-0.99$ & .70 & 0.30 & 74531 & \\
\hline $1.00-1.99$ & 1.41 & 0.30 & 27219 & \\
\hline $2.00-4.99$ & 3.16 & 0.40 & 31963 & \\
\hline $5.00-9.99$ & 7.07 & 0.30 & 2805 & \\
\hline$>10.00$ & 14.14 & 0.30 & 1624 & \\
\hline
\end{tabular}




\section{Aerosol Particle Measurement Record}

Description of sample location 3706 A Building, Resuspension Test, at .25 m, Date 8/11/94, Time sample started 7:47:09, Temperature 77.8 F, Humidity 41.3\%, Dew Point $52.7 \mathrm{~F}$, Average Wind Velocity (if applicable) $.00 \mathrm{~m} / \mathrm{s}$, \# of physical barriers between sample area and outside (if applicable) 1

Sample \#1

\begin{tabular}{|c|c|c|c|c|}
\hline $\begin{array}{l}\text { Bin } \\
\text { Range } \\
(\mu \mathrm{m})\end{array}$ & \begin{tabular}{|l} 
Bin Mean \\
Diameter \\
$($ (um)
\end{tabular} & $\begin{array}{l}\text { Diff. of the } \\
\log \text { of the } \\
\text { Size }\end{array}$ & Counts & $\begin{array}{l}\text { Weighted } \\
\text { Mean } \\
\text { Diameter }\end{array}$ \\
\hline $0.30 \cdot 0.49$ & 0.38 & 0.21 & 55086 & \multirow{6}{*}{0.97} \\
\hline $0.50-0.99$ & 0.70 & 0.30 & 31031 & \\
\hline $1.00-1.99$ & 1.41 & 0.30 & 11399 & \\
\hline $2.00-4.99$ & 3.16 & 0.40 & 12202 & \\
\hline $5,00-9.99$ & 7.07 & 0.30 & 908 & \\
\hline$>10.00$ & 14.14 & 0.30 & 232 & \\
\hline \multicolumn{5}{|l|}{ Sample \#3 } \\
\hline \begin{tabular}{|l} 
Bin \\
Range \\
(um)
\end{tabular} & \begin{tabular}{|l} 
Bin Mean \\
Diameter \\
$(\mu \mathrm{m})$
\end{tabular} & $\begin{array}{l}\text { Diff. of the } \\
\text { log of the } \\
\text { Size }\end{array}$ & Counts & $\begin{array}{l}\text { Weighted } \\
\text { Mean } \\
\text { Diameter }\end{array}$ \\
\hline $0.30-0.49$ & 0.38 & 0.21 & 48040 & \multirow{6}{*}{1.22} \\
\hline $0.50-0.99$ & 0.70 & 0.30 & 29238 & \\
\hline $1.00-1.99$ & 1.41 & 0.30 & 11972 & \\
\hline $2.00-4.99$ & 3.16 & 0.40 & 14859 & \\
\hline $5.00-9.99$ & 7.07 & 0.30 & 1808 & \\
\hline$>10.00$ & 14.14 & 0.30 & 1075 & \\
\hline \multicolumn{5}{|l|}{ Sample \#5 } \\
\hline $\begin{array}{l}\text { Bin } \\
\text { Range } \\
(\mu \mathrm{m}) \\
\end{array}$ & $\begin{array}{l}\text { Bin Mean } \\
\text { Diameter } \\
(\mu m)\end{array}$ & \begin{tabular}{|l} 
Diff. of the \\
log of the \\
Size
\end{tabular} & Counts & $\begin{array}{l}\text { Weighted } \\
\text { Mean } \\
\text { Diameter }\end{array}$ \\
\hline $0.30-0.49$ & 0.38 & 0.21 & 50671 & \multirow{6}{*}{1.10} \\
\hline $0.50 \cdot 0.99$ & 0.70 & 0.30 & 30365 & \\
\hline $1.00-1.99$ & 1.41 & 0.30 & 12086 & \\
\hline $2.00-4.99$ & 3.16 & 0.40 & 14119 & \\
\hline $5.00-9.99$ & 7.07 & 0.30 & 1474 & \\
\hline$>10.00$ & 14.14 & 0.30 & 574 & \\
\hline \multicolumn{5}{|l|}{ Sample \#7 } \\
\hline $\begin{array}{l}\text { Bin } \\
\text { Range } \\
\text { (um) }\end{array}$ & $\begin{array}{l}\text { Bin Mean } \\
\text { Diameter } \\
(\omega \mathrm{m})\end{array}$ & \begin{tabular}{|l|} 
Diff. of the \\
log of the \\
Size
\end{tabular} & Counts & $\begin{array}{l}\text { Weighted } \\
\text { Mean } \\
\text { Diameter }\end{array}$ \\
\hline $0.30-0.49$ & $\begin{array}{l}0.38 \\
\end{array}$ & 0.21 & 42790 & \multirow{6}{*}{1.38} \\
\hline $0.50-0.99$ & 0.70 & 0.30 & 32827 & \\
\hline $1.00-1.99$ & 1.41 & 0.30 & 14998 & \\
\hline $2.00 \cdot 4.99$ & 3.16 & 0.40 & 20980 & \\
\hline $5.00-9.99$ & 7.07 & 0.30 & 2489 & \\
\hline$>10.00$ & 14.14 & 0.30 & 1029 & \\
\hline \multicolumn{5}{|l|}{ Sample \#9 } \\
\hline $\begin{array}{l}\text { Bin } \\
\text { Range } \\
(\mu \mathrm{m}) \\
\end{array}$ & \begin{tabular}{|l} 
Bin Mean \\
Diameter \\
$(\mu \mathrm{m})$
\end{tabular} & $\begin{array}{l}\text { Diff. of the } \\
\log \text { of the } \\
\text { Size }\end{array}$ & Counts & $\begin{array}{l}\text { Weighted } \\
\text { Mean } \\
\text { Diameter }\end{array}$ \\
\hline $0.30-0.49$ & 0.38 & 0.21 & 30426 & \multirow{6}{*}{1.88} \\
\hline $0.50-0.99$ & 0.70 & 0.30 & 36149 & \\
\hline $1.00-1.99$ & 1.41 & 0.30 & 22506 & \\
\hline $2.00-4.99$ & 3.16 & 0.40 & 39356 & \\
\hline $5.00 \cdot 9.99$ & 7.07 & 0.30 & 5212 & \\
\hline$>10.00$ & 14.14 & 0.30 & 1734 & \\
\hline
\end{tabular}

Sample \#2

\begin{tabular}{||c|c|c|r|r||}
\hline $\begin{array}{l}\text { Bin } \\
\text { Range } \\
(\mu \mathrm{m})\end{array}$ & $\begin{array}{l}\text { Bin Mean } \\
\text { Diameter } \\
\text { (mm) }\end{array}$ & $\begin{array}{l}\text { Diff. of the } \\
\text { log of the } \\
\text { Size }\end{array}$ & Counts & $\begin{array}{l}\text { Weighted } \\
\text { Mean } \\
\text { Diameter }\end{array}$ \\
\hline $0.30-0.49$ & .38 & 0.21 & 15224 & \\
\hline $0.50-0.99$ & .70 & 0.30 & 13753 \\
\hline $1.00-1.99$ & 1.41 & 0.30 & 10315 \\
\hline $2.00-4.99$ & 3.16 & 0.40 & 48133 \\
\hline $5.00-9.99$ & 7.07 & 0.30 & 27552 \\
\hline$>10.00$ & 14.14 & 0.30 & 115020 \\
\hline
\end{tabular}

Sample \#4

\begin{tabular}{|c|c|c|c|c|}
\hline $\begin{array}{l}\text { Bin } \\
\text { Range } \\
\text { (um) }\end{array}$ & $\begin{array}{l}\text { Bin Mean } \\
\text { Diameter } \\
(u r m)\end{array}$ & $\begin{array}{l}\text { Diff. of the } \\
\text { log of the } \\
\text { Size }\end{array}$ & Counts & $\begin{array}{l}\text { Weighted } \\
\text { Mean } \\
\text { Diameter }\end{array}$ \\
\hline $0.30-0.49$ & .38 & 0.21 & 49352 & \multirow{6}{*}{1.16} \\
\hline $0.50-0.99$ & .70 & 0.30 & 30594 & \\
\hline $1.00-1.99$ & 1.41 & 0.30 & 12279 & \\
\hline $2.00-4.99$ & 3.16 & 0.40 & 14920 & \\
\hline $5.00-9.99$ & 7.07 & 0.30 & 1612 & \\
\hline$>10.00$ & 14.14 & 0.30 & 810 & \\
\hline
\end{tabular}
Sample \#6

\begin{tabular}{|c|c|c|c|c|}
\hline $\begin{array}{l}\text { Bin } \\
\text { Range } \\
(\mu \mathrm{m})\end{array}$ & \begin{tabular}{|l} 
Bin Mean \\
Diameter \\
$(\mu \mathrm{m})$
\end{tabular} & $\begin{array}{l}\text { Diff. of the } \\
\log \text { of the } \\
\text { Size }\end{array}$ & Counts & $\begin{array}{l}\text { Weighted } \\
\text { Mean } \\
\text { Diameter }\end{array}$ \\
\hline $0.30-0.49$ & .38 & 0.21 & 43641 & \multirow{6}{*}{1.35} \\
\hline $0.50-0.99$ & .70 & 0.30 & 33187 & \\
\hline $1.00-1.99$ & 1.41 & 0.30 & 15188 & \\
\hline $2.00-4.99$ & 3.16 & 0.40 & 20718 & \\
\hline $5.00-9.99$ & 7.07 & 0.30 & 2371 & \\
\hline$>10.00$ & 14.14 & 0.30 & 895 & \\
\hline
\end{tabular}

\begin{tabular}{|c|c|c|c|c|}
\hline \multicolumn{5}{|l|}{ Sample $\# 8$} \\
\hline $\begin{array}{l}\text { Bin } \\
\text { Range } \\
\text { (um) }\end{array}$ & $\begin{array}{l}\text { Bin Mean } \\
\text { Diameter } \\
(\mathrm{um})\end{array}$ & $\begin{array}{l}\text { Diff. of the } \\
\text { log of the } \\
\text { Size }\end{array}$ & Counts & $\begin{array}{l}\text { Weighted } \\
\text { Mean } \\
\text { Diameter }\end{array}$ \\
\hline $0.30-0.49$ & .38 & 0.21 & 30064 & \multirow{6}{*}{1.91} \\
\hline $0.50-0.99$ & .70 & 0.30 & 36070 & \\
\hline $0.00-1.99$ & 1.41 & 0.30 & 22828 & \\
\hline $2.00-4.99$ & 3.16 & 0.40 & 39990 & \\
\hline $5.00-9.99$ & 7.07 & 0.30 & 5519 & \\
\hline$>10.00$ & 14.14 & 0.30 & 1795 & \\
\hline
\end{tabular}

\begin{tabular}{|c|c|c|c|c|}
\hline \multicolumn{5}{|c|}{ Sample $\# 10$} \\
\hline $\begin{array}{l}\text { Bin } \\
\text { Range } \\
(\mu \mathrm{m})\end{array}$ & \begin{tabular}{|l} 
Bin Mean \\
Diameter \\
$(\mathrm{um})$
\end{tabular} & $\begin{array}{l}\text { Diff. of the } \\
\text { log of the } \\
\text { Size }\end{array}$ & Counts & $\begin{array}{l}\text { Weighted } \\
\text { Mean } \\
\text { Diamoter }\end{array}$ \\
\hline $0.30-0.49$ & .38 & 0.21 & 35118 & \multirow{6}{*}{1.66} \\
\hline $0.50-0.99$ & .70 & 0.30 & 34791 & \\
\hline $1.00-1.99$ & 1.41 & 0.30 & 19490 & \\
\hline $2.00-4.99$ & 3.16 & 0.40 & 30675 & \\
\hline $5.00-9.99$ & 7.07 & 0.30 & 4043 & \\
\hline$>10.00$ & 14.14 & 0.30 & 1277 & \\
\hline
\end{tabular}


Sample \#11

\begin{tabular}{|c|c|c|c|c|}
\hline $\begin{array}{l}\text { Bin } \\
\text { Range } \\
(\mu \mathrm{m})\end{array}$ & $\begin{array}{l}\text { Bin Mean } \\
\text { Diameter } \\
(\mu \mathrm{m})\end{array}$ & $\begin{array}{l}\text { Diff. of the } \\
\text { log of the } \\
\text { Size }\end{array}$ & Counts & $\begin{array}{l}\text { Weighted } \\
\text { Mean } \\
\text { Diameter }\end{array}$ \\
\hline $0.30-0.49$ & 0.38 & 0.21 & 34694 & \multirow{6}{*}{1.69} \\
\hline $0.50-0.99$ & 0.70 & 0.30 & 34156 & \\
\hline $1.00-1.99$ & 1.41 & 0.30 & 19357 & \\
\hline $2.00-4.99$ & 3.16 & 0.40 & 31235 & \\
\hline $5.00-9.99$ & 7.07 & 0.30 & 4190 & \\
\hline$>10.00$ & 14.14 & 0.30 & 1266 & \\
\hline
\end{tabular}

\begin{tabular}{|c|c|c|c|c|}
\hline \multicolumn{5}{|l|}{ Sample \#13 } \\
\hline $\begin{array}{l}\text { Bin } \\
\text { Range } \\
(\mathrm{mm})\end{array}$ & $\begin{array}{l}\text { Bin Mean } \\
\text { Diameter } \\
(\mu \mathrm{m})\end{array}$ & $\begin{array}{l}\text { Diff. of the } \\
\text { log of the } \\
\text { Size }\end{array}$ & Counts & $\begin{array}{l}\text { Weighted } \\
\text { Mean } \\
\text { Diameter }\end{array}$ \\
\hline $0.30-0.49$ & 0.38 & 0.21 & 43918 & \multirow{6}{*}{1.35} \\
\hline $0.50-0.99$ & 0.70 & 0.30 & 31767 & \\
\hline $1.00 \cdot 1.99$ & 1.41 & 0.30 & 14916 & \\
\hline $2.00-4.99$ & 3.16 & 0.40 & 20903 & \\
\hline $5.00-9.99$ & 7.07 & 0.30 & 2521 & \\
\hline$>10.00$ & 14.14 & 0.30 & 758 & \\
\hline
\end{tabular}

:
Sample \#12

\begin{tabular}{|c|c|c|c|c|}
\hline $\begin{array}{l}\text { Bin } \\
\text { Range } \\
\text { (um) }\end{array}$ & $\begin{array}{l}\text { Bin Mean } \\
\text { Diameter } \\
(\mu \mathrm{m})\end{array}$ & $\begin{array}{l}\text { Diff. of the } \\
\text { log of the } \\
\text { Size }\end{array}$ & Counts & $\begin{array}{l}\text { Weighted } \\
\text { Mean } \\
\text { Diameter }\end{array}$ \\
\hline $0.30-0.49$ & .38 & 0.21 & 40348 & \multirow{6}{*}{1.49} \\
\hline $0.50-0.99$ & .70 & 0.30 & 33104 & \\
\hline $1.00-1.99$ & 1.41 & 0.30 & 16936 & \\
\hline $2.00-4.99$ & 3.16 & 0.40 & 25233 & \\
\hline $5.00-9.99$ & 7.07 & 0.30 & 3200 & \\
\hline$>10.00$ & 14.14 & 0.30 & 955 & \\
\hline
\end{tabular}

\begin{tabular}{|c|c|c|c|c|}
\hline $\begin{array}{l}\text { Bin } \\
\text { Range } \\
(\mathrm{um})\end{array}$ & \begin{tabular}{|l|} 
Bin Mean \\
Diameter \\
$(u m)$
\end{tabular} & $\begin{array}{l}\text { Diff. of the } \\
\log \text { of the } \\
\text { Size }\end{array}$ & Counts & $\begin{array}{l}\text { Weighted } \\
\text { Mean } \\
\text { Diameter }\end{array}$ \\
\hline $0.30-0.49$ & .38 & 0.21 & 48136 & \multirow{6}{*}{1.21} \\
\hline $0.50-0.99$ & .70 & 0.30 & 31121 & \\
\hline $1.00-1.99$ & $\overline{1.41}$ & 0.30 & 13522 & \\
\hline $2.00-4.99$ & 3.16 & 0.40 & 18005 & \\
\hline $5.00-9.99$ & 7.07 & 0.30 & 1941 & \\
\hline$>10.00$ & 14.14 & 0.30 & 499 & \\
\hline
\end{tabular}


Data for Fig. 4 Resuspension and Ambient Air Vs. Height, Building 3706, Room 102

Table 6. Peak Mean Diameter $(\mu \mathrm{m})$, Versus Sampling Height,

and

Table 8. Percent of Large Particles in Breathing Zone, Building

3706, Room 102

$0.5 \mathrm{~m}$

$1.0 \mathrm{~m}$

$1.5 \mathrm{~m}$

$2.0 \mathrm{~m}$ with

with

with

with
$0.25 \mathrm{~m}$

$0.25 \mathrm{~m}$

$0.25 \mathrm{~m}$

$0.25 \mathrm{~m}$ 


\section{Aerosol Particle Measurement Record}

Description of sample location 3706 Building, Resuspension Test, at $0.5 \mathrm{~m}$, Date 8/26/94, Time sample started 14:46:18, Temperature $78.9 \mathrm{~F}$, Humidity $36.1 \%$, Dew Point $50.0 \mathrm{~F}$ - Average Wind Velocity (if applicable) $.00 \mathrm{~m} / \mathrm{s}$, \# of physical barriers between sample area and outside (if applicable) $\underline{2}$

Sample \#1

\begin{tabular}{|c|c|c|c|c|}
\hline \begin{tabular}{|l|} 
Bin \\
Range \\
$(\mu \mathrm{m})$
\end{tabular} & \begin{tabular}{|l|} 
Bin Mean \\
Diameter \\
(um)
\end{tabular} & $\begin{array}{l}\text { Diff. of the } \\
\text { log of the } \\
\text { Size }\end{array}$ & Counts & $\begin{array}{l}\text { Weighted } \\
\text { Mean } \\
\text { Diameter }\end{array}$ \\
\hline $0.30-0.49$ & 0.38 & 0.21 & 32548 & \multirow{6}{*}{0.72} \\
\hline $0.50-0.99$ & 0.70 & 0.30 & 9432 & \\
\hline $1.00-1.99$ & 1.41 & 0.30 & 2343 & \\
\hline $2.00-4.99$ & 3.16 & 0.40 & 2217 & \\
\hline $5.00-9.99$ & 7.07 & 0.30 & 202 & \\
\hline$>10.00$ & 14.14 & 0.30 & 205 & \\
\hline \multicolumn{5}{|l|}{ Sample \#3 } \\
\hline \begin{tabular}{|l|} 
Bin \\
Range \\
$(\mu \mathrm{m})$
\end{tabular} & $\begin{array}{l}\text { Bin Mean } \\
\text { Diameter } \\
(u \mathrm{~m})\end{array}$ & \begin{tabular}{|l|} 
Diff. of the \\
log of the \\
Size
\end{tabular} & Counts & $\begin{array}{l}\text { Weighted } \\
\text { Mean } \\
\text { Diameter }\end{array}$ \\
\hline $0.30-0.49$ & 0.38 & 0.21 & 22365 & \\
\hline $0.50 \cdot 0.99$ & 0.70 & 0.30 & 10177 & \\
\hline $1.00-1.99$ & 1.41 & 0.30 & 4591 & \\
\hline $2.00-4.99$ & 3.16 & 0.40 & 9289 & \\
\hline $5.00-9.99$ & 7.07 & 0.30 & 2332 & \\
\hline$>10.00$ & 14.14 & 0.30 & 2210 & \\
\hline \multicolumn{5}{|l|}{ Sample \#5 } \\
\hline \begin{tabular}{|l|} 
Bin \\
Range \\
$(\mu \mathrm{m})$
\end{tabular} & \begin{tabular}{|l|}
$\begin{array}{l}\text { Bin Mean } \\
\text { Diameter } \\
(\mu m)\end{array}$ \\
\end{tabular} & $\begin{array}{l}\text { Diff. of the } \\
\log \text { of the } \\
\text { Size }\end{array}$ & Counts & \begin{tabular}{|l|} 
Weighted \\
Mean \\
Diameter
\end{tabular} \\
\hline $0.30-0.49$ & 0.38 & \begin{tabular}{|l|}
0.21 \\
\end{tabular} & 25962 & \multirow{6}{*}{1.42} \\
\hline $0.50-0.99$ & 0.70 & 0.30 & 9663 & \\
\hline $1.00-1.99$ & 1.41 & 0.30 & 3535 & \\
\hline $2.00 \cdot 4.99$ & 3.16 & 0.40 & 6083 & \\
\hline $5.00-9.99$ & 7.07 & 0.30 & 1373 & \\
\hline$>10.00$ & 14.14 & 0.30 & 1216 & \\
\hline \multicolumn{5}{|l|}{ Sample $\# 7$} \\
\hline $\begin{array}{l}\text { Bin } \\
\text { Range } \\
(\mu \mathrm{m}) \\
\end{array}$ & $\begin{array}{l}\text { Bin Mean } \\
\text { Diameter } \\
(\mu \mathrm{m})\end{array}$ & \begin{tabular}{|l|} 
Diff. of the \\
log of the \\
Size
\end{tabular} & Counts & $\begin{array}{l}\text { Weighted } \\
\text { Mean } \\
\text { Diameter }\end{array}$ \\
\hline $0.30-0.49$ & 0.38 & $\begin{array}{l}0.21 \\
\end{array}$ & 28916 & \multirow{6}{*}{1.06} \\
\hline $0.50-0.99$ & 0.70 & 0.30 & 9233 & \\
\hline $1.00-1.99$ & 1.41 & 0.30 & 2833 & \\
\hline $2.00-4.99$ & 3.16 & 0.40 & 3937 & \\
\hline $5.00-9.99$ & 7.07 & 0.30 & 841 & \\
\hline$>10.00$ & 14.14 & 0.30 & 666 & \\
\hline \multicolumn{5}{|l|}{ ample \#9 } \\
\hline $\begin{array}{l}\text { Bin } \\
\text { Range } \\
(\mathrm{rm})\end{array}$ & $\begin{array}{l}\text { Bin Mean } \\
\text { Diameter } \\
(\mu \mathrm{m})\end{array}$ & \begin{tabular}{|l|} 
Diff. of the \\
log of the \\
Size
\end{tabular} & Counts & $\begin{array}{l}\text { Weighted } \\
\text { Mean } \\
\text { Diameter }\end{array}$ \\
\hline $0.30-0.49$ & 0.38 & 0.21 & 30073 & \multirow{6}{*}{0.91} \\
\hline $0.50 \cdot 0.99$ & 0.70 & 0.30 & 9186 & \\
\hline $1.00-1.99$ & 1.41 & 0.30 & 2586 & \\
\hline $2.00-4.99$ & 3.16 & 0.40 & 3197 & \\
\hline $5.00-9.99$ & 7.07 & 0.30 & 556 & \\
\hline$>10.00$ & 14.14 & 0.30 & 438 & \\
\hline
\end{tabular}

Sample \#2

\begin{tabular}{|c|c|c|c|c|}
\hline $\begin{array}{l}\text { Bin } \\
\text { Range } \\
\text { (um) }\end{array}$ & \begin{tabular}{|l|} 
Bin Mean \\
Diameter \\
$(u m)$
\end{tabular} & $\begin{array}{l}\text { Diff. of the } \\
\text { log of the } \\
\text { Size }\end{array}$ & Counts & $\begin{array}{l}\text { Weighted } \\
\text { Mean } \\
\text { Diameter }\end{array}$ \\
\hline $0.30-0.49$ & .38 & 0.21 & 26244 & \multirow{6}{*}{1.48} \\
\hline $0.50-0.99$ & .70 & 0.30 & 10272 & \\
\hline $1.00-1.99$ & 1.41 & 0.30 & 3751 & \\
\hline $2.00-4.99$ & 3.16 & 0.40 & 6339 & \\
\hline $5.00-9.99$ & 7.07 & 0.30 & 1393 & \\
\hline$>10.00$ & 14.14 & 0.30 & 1486 & \\
\hline
\end{tabular}

Sample \#4

\begin{tabular}{|c|c|c|c|c|}
\hline $\begin{array}{l}\text { Bin } \\
\text { Range } \\
(\mathrm{mm})\end{array}$ & \begin{tabular}{|l|} 
Bin Mean \\
Diameter \\
$(\mathrm{um})$
\end{tabular} & $\begin{array}{l}\text { Diff. of the } \\
\text { log of the } \\
\text { Size }\end{array}$ & Counts & $\begin{array}{l}\text { Weighted } \\
\text { Mean } \\
\text { Diameter }\end{array}$ \\
\hline $0.30-0.49$ & .38 & 0.21 & 25869 & \multirow{6}{*}{1.46} \\
\hline $0.50-0.99$ & .70 & 0.30 & 9789 & \\
\hline $1.00-1.99$ & 1.41 & 0.30 & 3753 & \\
\hline $2.00-4.99$ & 3.16 & $0.40^{\circ}$ & 6228 & \\
\hline $5.00-9.99$ & 7.07 & 0.30 & 1418 & \\
\hline$>10.00$ & 14.14 & 0.30 & 1345 & \\
\hline
\end{tabular}

\begin{tabular}{|c|c|c|c|c|}
\hline \multicolumn{5}{|c|}{ Sample \#6 } \\
\hline $\begin{array}{l}\text { Bin } \\
\text { Range } \\
\text { (um) }\end{array}$ & \begin{tabular}{|l} 
Bin Mean \\
Diameter \\
$(u m)$
\end{tabular} & $\begin{array}{l}\text { Diff. of the } \\
\text { log of the } \\
\text { Size }\end{array}$ & Counts & $\begin{array}{l}\text { Woighted } \\
\text { Mean } \\
\text { Diameter }\end{array}$ \\
\hline $0.30-0.49$ & .38 & 0.21 & 28063 & \multirow{6}{*}{1.19} \\
\hline $0.50-0.99$ & .70 & 0.30 & 9325 & \\
\hline $1.00-1.99$ & 1.41 & 0.30 & 3049 & \\
\hline $2.00-4.99$ & 3.16 & 0.40 & 4601 & \\
\hline$\cdot 5.00-9.99$ & 7.07 & 0.30 & 987 & \\
\hline$>10.00$ & 14.14 & 0.30 & 894 & \\
\hline
\end{tabular}

\begin{tabular}{|c|c|c|c|c|}
\hline \multicolumn{5}{|c|}{ Sample \#8 } \\
\hline $\begin{array}{l}\text { Bin } \\
\text { Range } \\
(\mu \mathrm{m})\end{array}$ & \begin{tabular}{|l} 
Bin Mean \\
Diameter \\
$(\mu \mathrm{m})$
\end{tabular} & $\begin{array}{l}\text { Diff. of the } \\
\text { log of the } \\
\text { Size }\end{array}$ & Counts & $\begin{array}{l}\text { Weighted } \\
\text { Mean } \\
\text { Diameter }\end{array}$ \\
\hline $0.30-0.49$ & .38 & 0.21 & 28748 & \multirow{6}{*}{1.02} \\
\hline $0.50-0.99$ & .70 & 0.30 & 9104 & \\
\hline $0.00-1.99$ & 1.41 & 0.30 & 2781 & \\
\hline $2.00-4.99$ & 3.16 & 0.40 & 3731 & \\
\hline $5.00-9.99$ & 7.07 & 0.30 & 721 & \\
\hline$>10.00$ & 14.14 & 0.30 & 617 & \\
\hline
\end{tabular}

\begin{tabular}{|c|c|c|c|c|}
\hline \multicolumn{5}{|c|}{ Sample $\# 10$} \\
\hline \begin{tabular}{|l} 
Bin \\
Range \\
$(\mu \mathrm{m})$ \\
\end{tabular} & $\begin{array}{l}\text { Bin Mean } \\
\text { Diameter } \\
(u m)\end{array}$ & $\begin{array}{l}\text { Diff. of the } \\
\text { log of the } \\
\text { Size }\end{array}$ & Counts & $\begin{array}{l}\text { Weighted } \\
\text { Mean } \\
\text { Diameter }\end{array}$ \\
\hline $0.30-0.49$ & .38 & 0.21 & 30605 & \multirow{6}{*}{0.85} \\
\hline $0.50-0.99$ & .70 & 0.30 & 8904 & \\
\hline $1.00-1.99$ & 1.41 & 0.30 & 2506 & \\
\hline $2.00-4.99$ & 3.16 & 0.40 & 2971 & \\
\hline $5.00-9.99$ & 7.07 & 0.30 & 471 & \\
\hline$>10.00$ & 14.14 & 0.30 & 356 & \\
\hline
\end{tabular}




\section{Aerosol Particle Measurement Record}

Description of sample location 3706 Building, Resuspension Test, at $0.25 \mathrm{~m}$, Date 8/26/94, Time sample started 14:46:18, Temperature 78.9 F, Humidity $36.1 \%$, Dew Point $50.0 \mathrm{~F}$, Average Wind Velocity (if applicable) $.00 \mathrm{~m} / \mathrm{s}$, \# of physical barriers between sample area and outside (if applicable) $2 \underline{2}$

\begin{tabular}{|c|c|c|c|c|}
\hline $\begin{array}{l}\text { Bin } \\
\text { Range } \\
\text { (urm) }\end{array}$ & $\begin{array}{l}\text { Bin Mean } \\
\text { Diameter } \\
(\mathrm{um})\end{array}$ & $\begin{array}{l}\text { Diff. of the } \\
\text { log of the } \\
\text { Size }\end{array}$ & Counts & $\begin{array}{l}\text { Weighted } \\
\text { Mean } \\
\text { Diameter }\end{array}$ \\
\hline $0.30-0.49$ & 0.38 & 0.21 & 31599 & \multirow{6}{*}{ a. 0.72} \\
\hline $0.50-0.99$ & 0.70 & 0.30 & 21293 & \\
\hline $1.00-1.99$ & 1.41 & 0.30 & 2456 & \\
\hline $2.00-4.99$ & 3.16 & 0.40 & 2177 & \\
\hline $5.00-9.99$ & 7.07 & 0.30 & 232 & \\
\hline$>10.00$ & 14.14 & 0.30 & 209 & \\
\hline \multicolumn{5}{|l|}{ Sample $\$ 3$} \\
\hline \begin{tabular}{|l} 
Bin \\
Range \\
$(\mu \mathrm{m})$
\end{tabular} & $\begin{array}{l}\text { Bin Mean } \\
\text { Diameter } \\
(u m)\end{array}$ & $\begin{array}{l}\text { Diff. of the } \\
\log \text { of the } \\
\text { Size }\end{array}$ & Counts & $\begin{array}{l}\text { Weighted } \\
\text { Mean } \\
\text { Diameter }\end{array}$ \\
\hline $0.30-0.49$ & 0.38 & 0.21 & 14459 & \multirow{6}{*}{. 2.56} \\
\hline $0.50-0.99$ & 0.70 & 0.30 & 18818 & \\
\hline $1.00-1.99$ & 1.41 & 0.30 & 7499 & \\
\hline $2.00 \cdot 4.99$ & 3.16 & 0.40 & 15862 & \\
\hline $5.00-9.99$ & 7.07 & 0.30 & 4064 & \\
\hline$>10.00$ & 14.14 & 0.30 & 4087 & \\
\hline \multicolumn{5}{|l|}{ Sample \#5 } \\
\hline \begin{tabular}{|l} 
Bin \\
Range \\
$(\mu \mathrm{m})$
\end{tabular} & \begin{tabular}{|l}
$\begin{array}{l}\text { Bin Mean } \\
\text { Diameter } \\
(u m)\end{array}$ \\
\end{tabular} & $\begin{array}{l}\text { Diff. of the } \\
\text { log of the } \\
\text { Size }\end{array}$ & Counts & $\begin{array}{l}\text { Weighted } \\
\text { Mean } \\
\text { Diameter }\end{array}$ \\
\hline $0.30-0.49$ & 0.38 & 0.21 & 26566 & \multirow{6}{*}{1.17} \\
\hline $0.50-0.99$ & 0.70 & 0.30 & 19923 & \\
\hline $1.00-1.99$ & 1.41 & 0.30 & 3360 & \\
\hline $2.00-4.99$ & 3.16 & 0.40 & 4871 & \\
\hline $5.00-9.99$ & 7.07 & 0.30 & 950 & \\
\hline$>10.00$ & 14.14 & 0.30 & 1093 & \\
\hline \multicolumn{5}{|l|}{ Sample \#7 } \\
\hline \begin{tabular}{|l} 
Bin \\
Range \\
$(u \mathrm{~m})$
\end{tabular} & \begin{tabular}{|l} 
Bin Mean \\
Diameter \\
$($ (um)
\end{tabular} & $\begin{array}{l}\text { Diff. of the } \\
\log \text { of the } \\
\text { Size }\end{array}$ & Counts & $\begin{array}{l}\text { Weighted } \\
\text { Mean } \\
\text { Diameter }\end{array}$ \\
\hline $0.30-0.49$ & 0.38 & 0.21 & 28875 & \multirow{6}{*}{0.90} \\
\hline $0.50-0.99$ & 0.70 & 0.30 & 20066 & \\
\hline $1.00-1.99$ & 1.41 & 0.30 & 2581 & \\
\hline $2.00-4.99$ & 3.16 & 0.40 & 3241 & \\
\hline $5.00-9.99$ & 7.07 & 0.30 & 522 & \\
\hline$>10.00$ & 14.14 & 0.30 & 533 & \\
\hline \multicolumn{5}{|l|}{ Sample \#9 } \\
\hline \begin{tabular}{|l} 
Bin \\
Range \\
$(u m)$
\end{tabular} & $\begin{array}{l}\text { Bin Mean } \\
\text { Diameter } \\
(u m)\end{array}$ & \begin{tabular}{|l|} 
Diff. of the \\
log of the \\
Size
\end{tabular} & Counts & \begin{tabular}{|l|} 
Weighted \\
Mean \\
Diameter
\end{tabular} \\
\hline $0.30-0.49$ & 0.38 & 0.21 & 37088 & \multirow{6}{*}{0.76} \\
\hline $0.50-0.99$ & 0.70 & 0.30 & 21944 & \\
\hline $1.00-1.99$ & 1.41 & 0.30 & 2436 & \\
\hline $2.00-4.99$ & 3.16 & 0.40 & 2548 & \\
\hline $5.00-9.99$ & 7.07 & 0.30 & 349 & \\
\hline$>10.00$ & 14.14 & 0.30 & 391 & \\
\hline
\end{tabular}

\begin{tabular}{|c|c|c|c|c|}
\hline $\begin{array}{l}\text { Bin } \\
\text { Range } \\
(\mu \mathrm{m})\end{array}$ & \begin{tabular}{|l} 
Bin Mean \\
Diameter \\
$(\mathrm{em})$
\end{tabular} & $\begin{array}{l}\text { Diff, of the } \\
\text { log of the } \\
\text { Size }\end{array}$ & Counts & $\begin{array}{l}\text { Weighted } \\
\text { Mean } \\
\text { Diameter }\end{array}$ \\
\hline $0.30-0.49$ & .38 & 0.21 & 12257 & \\
\hline $0.50-0.99$ & .70 & 0.30 & 20305 & \\
\hline $1.00-1.99$ & 1.41 & 0.30 & 10208 & \\
\hline $2.00-4.99$ & 3.16 & $\overline{0.40}$ & 25461 & \\
\hline $5.00-9.99$ & 7.07 & 0.30 & 7110 & \\
\hline$>10.00$ & 74.14 & 0.30 & 10153 & \\
\hline
\end{tabular}

\begin{tabular}{|c|c|c|c|c|}
\hline \multicolumn{5}{|l|}{ Sample \#4 } \\
\hline $\begin{array}{l}\text { Bin } \\
\text { Range } \\
\text { (um) }\end{array}$ & $\begin{array}{l}\text { Bin Mean } \\
\text { Diameter } \\
(\mathrm{um})\end{array}$ & $\begin{array}{l}\text { Diff. of the } \\
\text { log of the } \\
\text { Size }\end{array}$ & Counts & $\begin{array}{l}\text { Weighted } \\
\text { Mean } \\
\text { Diameter }\end{array}$ \\
\hline $0.30-0.49$ & .38 & 0.21 & 24236 & \multirow{6}{*}{1.53} \\
\hline $0.50-0.99$ & .70 & 0.30 & 20791 & \\
\hline $1.00-1.99$ & 1.41 & 0.30 & 4750 & \\
\hline $2.00-4.99$ & 3.16 & 0.40 & 8338 & \\
\hline $5.00-9.99$ & 7.07 & 0.30 & 1890 & \\
\hline$>10.00$ & 14.14 & 0.30 & 1719 & \\
\hline
\end{tabular}

\begin{tabular}{|c|c|c|c|c|}
\hline $\begin{array}{l}\text { Bin } \\
\text { Range } \\
\text { (um) }\end{array}$ & $\begin{array}{l}\text { Bin Mean } \\
\text { Diameter } \\
(\mu \mathrm{m})\end{array}$ & $\begin{array}{l}\text { Diff, of the } \\
\log \text { of the } \\
\text { Size }\end{array}$ & Counts & $\begin{array}{l}\text { Woighted } \\
\text { Mean } \\
\text { Diameter }\end{array}$ \\
\hline $0.30-0.49$ & .38 & 0.21 & 28657 & \multirow{6}{*}{0.96} \\
\hline $0.50-0.99$ & .70 & 0.30 & 20308 & \\
\hline $1.00-1.99$ & 1.41 & 0.30 & 2742 & \\
\hline $2.00-4.99$ & 3.16 & 0.40 & 3490 & \\
\hline $5.00-9.99$ & 7.07 & 0.30 & 593 & \\
\hline$>10.00$ & 14.14 & 0.30 & 728 & \\
\hline
\end{tabular}

Sample \#8

\begin{tabular}{|c|c|c|c|c|}
\hline $\begin{array}{l}\text { Bin } \\
\text { Range } \\
\text { (um) }\end{array}$ & \begin{tabular}{|l} 
Bin Mean \\
Diameter \\
$(\mu \mathrm{m})$
\end{tabular} & $\begin{array}{l}\text { Diff. of the } \\
\text { log of the } \\
\text { Size }\end{array}$ & Counts & $\begin{array}{l}\text { Weighted } \\
\text { Mean } \\
\text { Diameter }\end{array}$ \\
\hline $0.30-0.49$ & .38 & 0.21 & 30780 & \multirow{6}{*}{0.81} \\
\hline $0.50-0.99$ & .70 & 0.30 & 20337 & \\
\hline $0.00-1.99$ & 1.41 & 0.30 & 2293 & \\
\hline $2.00-4.99$ & 3.16 & 0.40 & 2417 & \\
\hline $5.00-9.99$ & 7.07 & 0.30 & 414 & \\
\hline$>10.00$ & 14.14 & 0.30 & 416 & \\
\hline
\end{tabular}

\begin{tabular}{|c|c|c|c|c|}
\hline \multicolumn{5}{|c|}{ Sample \#10 } \\
\hline $\begin{array}{l}\text { Bin } \\
\text { Range } \\
(\mu \mathrm{m})\end{array}$ & \begin{tabular}{|l} 
Bin Mean \\
Diameter \\
$(\mu \mathrm{m})$
\end{tabular} & $\begin{array}{l}\text { Diff. of the } \\
\text { log of the } \\
\text { Size }\end{array}$ & Counts & $\begin{array}{l}\text { Weighted } \\
\text { Mean } \\
\text { Diameter }\end{array}$ \\
\hline $0.30-0.49$ & .38 & 0.21 & 30596 & \multirow{6}{*}{0.78} \\
\hline $0.50-0.99$ & .70 & 0.30 & 20027 & \\
\hline $1.00-1.99$ & 1.41 & 0.30 & 2285 & \\
\hline $2.00-4.99$ & 3.16 & 0.40 & 2421 & \\
\hline $5.00-9.99$ & 7.07 & 0.30 & 380 & \\
\hline$>10.00$ & 14.14 & 0.30 & 327 & \\
\hline
\end{tabular}




\section{Aerosol Particle Measurement Record}

Description of sample location 3706 Building, Resuspension Test, at $1.0 \mathrm{~m}$, Date $8 / 26 / 94$, Time sample started_14:01:18, Temperature_77.9 F, Humidity 37.2\%, Dew Point 49.9 F , Average Wind Velocity (if applicable) $.00 \mathrm{~m} / \mathrm{s}$, \# of physical barriers between sample area and outside (if applicable) 2

\begin{tabular}{|c|c|c|c|c|}
\hline $\begin{array}{l}\text { Bin } \\
\text { Range } \\
\text { (um) }\end{array}$ & \begin{tabular}{|l|} 
Bin Mean \\
Diameter \\
$($ (rm)
\end{tabular} & $\begin{array}{l}\text { Diff. of the } \\
\text { log of the } \\
\text { Size }\end{array}$ & Counts & $\begin{array}{l}\text { Weighted } \\
\text { Mean } \\
\text { Diameter }\end{array}$ \\
\hline $0.30-0.49$ & 0.38 & 0.21 & 37461 & \multirow{6}{*}{0.70} \\
\hline $0.50-0.99$ & 0.70 & 0.30 & 12317 & \\
\hline $1.00-1.99$ & 1.41. & 0.30 & 3133 & \\
\hline $2.00-4.99$ & 3.16 & 0.40 & 2597 & \\
\hline $5.00-9.99$ & 7.07 & 0.30 & 188 & \\
\hline$>10.00$ & 14.14 & 0.30 & 168 & \\
\hline \multicolumn{5}{|l|}{ Sample \#3 } \\
\hline \begin{tabular}{|l} 
Bin \\
Range \\
$(\mu \mathrm{m})$
\end{tabular} & \begin{tabular}{|l} 
Bin Mean \\
Diameter \\
$(u m)$
\end{tabular} & \begin{tabular}{|l|} 
Diff. of the \\
log of the \\
Size
\end{tabular} & Counts & \begin{tabular}{|l} 
Weighted \\
Mean \\
Diameter
\end{tabular} \\
\hline $0.30-0.49$ & $\begin{array}{l}0.38 \\
\end{array}$ & 0.21 & 20032 & \\
\hline $0.50-0.99$ & 0.70 & 0.30 & 14579 & \\
\hline $1.00-1.99$ & 1.41 & 0.30 & 8052 & \\
\hline $2.00-4.99$ & 3.16 & 0.40 & 17134 & \\
\hline $5.00 \cdot 9.99$ & 7.07 & 0.30 & 3842 & \\
\hline$>10.00$ & 94.14 & 0.30 & 3258 & \\
\hline \multicolumn{5}{|l|}{ Sample \#5 } \\
\hline \begin{tabular}{|l} 
Bin \\
Range \\
$(\mu \mathrm{m})$
\end{tabular} & \begin{tabular}{|l} 
Bin Mean \\
Diameter \\
$(\mu \mathrm{m})$
\end{tabular} & $\begin{array}{l}\text { Diff. of the } \\
\text { log of the } \\
\text { Size }\end{array}$ & Counts & \begin{tabular}{|l|} 
Weighted \\
Mean \\
Diameter
\end{tabular} \\
\hline $0.30 \cdot 0.49$ & 0.38 & 0.21 & 27851 & \multirow{6}{*}{1.49} \\
\hline $0.50-0.99$ & 0.70 & 0.30 & 13115 & \\
\hline $1.00-1.99$ & 1.41 & 0.30 & 5406 & \\
\hline $2.00-4.99$ & 3.16 & 0.40 & 8964 & \\
\hline $5.00-9.99$ & 7.07 & 0.30 & 1711 & \\
\hline$>10.00$ & 14.14 & 0.30 & 1344 & \\
\hline \multicolumn{5}{|l|}{ Sample \#7 } \\
\hline \begin{tabular}{|l} 
Bin \\
Range \\
$(\mathrm{um})$ \\
\end{tabular} & $\begin{array}{l}\text { Bin Mean } \\
\text { Diameter } \\
(\mathrm{mm})\end{array}$ & \begin{tabular}{|l|}
$\begin{array}{l}\text { Diff. of the } \\
\text { log of the } \\
\text { Size }\end{array}$ \\
\end{tabular} & Counts & \begin{tabular}{|l|}
$\begin{array}{l}\text { Weighted } \\
\text { Mean } \\
\text { Diameter }\end{array}$ \\
\end{tabular} \\
\hline $0.30-0.49$ & 0.38 & 0.21 & 31195 & \multirow{6}{*}{1.15} \\
\hline $0.50 \cdot 0.99$ & 0.70 & 0.30 & 12276 & \\
\hline $1.00-1.99$ & 1.41 & 0.30 & 4234 & \\
\hline $2.00 \cdot 4.99$ & 3.16 & 0.40 & 6048 & \\
\hline $5.00-9.99$ & 7.07 & 0.30 & 1031 & \\
\hline$>10.00$ & 14.14 & 0.30 & 794 & \\
\hline \multicolumn{5}{|l|}{ Sample \#9 } \\
\hline $\begin{array}{l}\text { Bin } \\
\text { Range } \\
\text { (um) } \\
\end{array}$ & \begin{tabular}{|l|} 
Bin Mean \\
Diameter \\
$(\mathrm{um})$
\end{tabular} & \begin{tabular}{|l|} 
Diff. of the \\
log of the \\
Size
\end{tabular} & Counts & $\begin{array}{l}\text { Weighted } \\
\text { Mean } \\
\text { Diameter }\end{array}$ \\
\hline $0.30 \cdot 0.49$ & 0.38 & 0.21 & 33644 & \multirow{6}{*}{0.93} \\
\hline $0.50-0.99$ & 0.70 & 0.30 & 11775 & \\
\hline $1.00-1.99$ & 1.41 & 0.30 & 3571 & \\
\hline $2.00-4.99$ & 3.16 & 0.40 & 4199 & \\
\hline $5.00 \cdot 9.99$ & 7.07 & 0.30 & 636 & \\
\hline$>10.00$ & 14.14 & 0.30 & 485 & \\
\hline
\end{tabular}

Sample \#2

\begin{tabular}{|c|c|c|c|c|}
\hline $\begin{array}{l}\text { Bin } \\
\text { Range } \\
\text { (um) }\end{array}$ & \begin{tabular}{|l} 
Bin Mean \\
Diameter \\
$(\mu \mathrm{m})$
\end{tabular} & $\begin{array}{l}\text { Diff. of the } \\
\text { log of the } \\
\text { Size }\end{array}$ & Counts & $\begin{array}{l}\text { Weighted } \\
\text { Mean } \\
\text { Diameter }\end{array}$ \\
\hline $0.30-0.49$ & .38 & 0.21 & 37235 & \multirow{6}{*}{0.73} \\
\hline $0.50-0.99$ & .70 & 0.30 & 12532 & \\
\hline $1.00-1.99$ & 1.41 & 0.30 & 3263 & \\
\hline $2.00-4.99$ & 3.16 & 0.40 & 2783 & \\
\hline $5.00-9.99$ & 7.07 & 0.30 & 276 & \\
\hline$>10.00$ & 14.14 & 0.30 & 190 & \\
\hline
\end{tabular}
Sample \#4

\begin{tabular}{|c|c|c|c|c|}
\hline $\begin{array}{l}\text { Bin } \\
\text { Range } \\
\text { (um) }\end{array}$ & $\begin{array}{l}\text { Bin Mean } \\
\text { Diameter } \\
(\mathrm{em})\end{array}$ & $\begin{array}{l}\text { Diff. of the } \\
\text { log of the } \\
\text { Size }\end{array}$ & Counts & $\begin{array}{l}\text { Weighted } \\
\text { Mean } \\
\text { Diameter }\end{array}$ \\
\hline $0.30-0.49$ & .38 & 0.21 & 23284 & \multirow{6}{*}{1.93} \\
\hline $0.50-0.99$ & .70 & 0.30 & 13484 & \\
\hline $1.00-1.99$ & 1.41 & 0.30 & 6641 & \\
\hline $2.00-4.99$ & 3.16 & 0.40 & 13264 & \\
\hline $5.00-9.99$ & 7.07 & 0.30 & 2734 & \\
\hline$>10.00$ & 14.14 & 0.30 & 2095 & \\
\hline
\end{tabular}

Sample \#6

\begin{tabular}{|c|c|c|c|c|}
\hline $\begin{array}{l}\text { Bin } \\
\text { Range } \\
\text { (um) }\end{array}$ & $\begin{array}{l}\text { Bin Mean } \\
\text { Diameter } \\
(\mu \mathrm{m})\end{array}$ & $\begin{array}{l}\text { Diff. of the } \\
\log \text { of the } \\
\text { Size }\end{array}$ & Counts & $\begin{array}{l}\text { Weighted } \\
\text { Mean } \\
\text { Diameter }\end{array}$ \\
\hline $0.30-0.49$ & .38 & 0.21 & 29569 & \multirow{6}{*}{1.31} \\
\hline $0.50-0.99$ & .70 & 0.30 & 12785 & \\
\hline $1.00-1.99$ & 1.41 & 0.30 & 4727 & \\
\hline $2.00-4.99$ & $\overline{3.16}$ & 0.40 & $\overline{7379}$ & \\
\hline $5.00-9.99$ & 7.07 & 0.30 & 1320 & \\
\hline$>10.00$ & 94.14 & 0.30 & 1058 & \\
\hline
\end{tabular}

\section{Sample \#8}

\begin{tabular}{|c|c|c|c|c|}
\hline $\begin{array}{l}\text { Bin } \\
\text { Range } \\
(\mu \mathrm{m})\end{array}$ & \begin{tabular}{|l} 
Bin Mean \\
Diameter \\
$(\mu \mathrm{m})$
\end{tabular} & $\begin{array}{l}\text { Diff. of the } \\
\text { log of the } \\
\text { Size }\end{array}$ & Counts & $\begin{array}{l}\text { Weighted } \\
\text { Mean } \\
\text { Diameter }\end{array}$ \\
\hline $0.30-0.49$ & .38 & 0.21 & 32843 & \multirow{6}{*}{1.01} \\
\hline $0.50-0.99$ & .70 & 0.30 & 12225 & \\
\hline $0.00-1.99$ & 1.41 & 0.30 & 3773 & \\
\hline $2.00-4.99$ & 3.16 & 0.40 & 4860 & \\
\hline $5.00-9.99$ & 7.07 & 0.30 & 819 & \\
\hline$>10.00$ & 14.14 & 0.30 & 576 & \\
\hline
\end{tabular}

\begin{tabular}{|c|c|c|c|c|}
\hline \multicolumn{5}{|c|}{ Sample \#10 } \\
\hline $\begin{array}{l}\text { Bin } \\
\text { Range } \\
\text { (uml }\end{array}$ & \begin{tabular}{|l} 
Bin Mean \\
Diameter \\
(um)
\end{tabular} & $\begin{array}{l}\text { Diff. of the } \\
\text { log of the } \\
\text { Size }\end{array}$ & Counts & $\begin{array}{l}\text { Weighted } \\
\text { Mean } \\
\text { Diameter }\end{array}$ \\
\hline $0.30-0.49$ & .38 & 0.21 & 33934 & \multirow{6}{*}{0.88} \\
\hline $0.50-0.99$ & .70 & 0.30 & 11761 & \\
\hline $1.00-1.99$ & 1.41 & 0.30 & 3429 & \\
\hline $2.00-4.99$ & 3.16 & 0.40 & 3885 & \\
\hline $5.00-9.99$ & 7.07 & 0.30 & 557 & \\
\hline$>10.00$ & 14.14 & 0.30 & 359 & \\
\hline
\end{tabular}




\section{Aerosol Particle Measurement Record}

Description of sample location 3706 Building, Resuspension Test, at $.25 \mathrm{~m}$, Date 8/26/94, Time sample started $14: 01: 18$, Temperature $77.9 \mathrm{~F}$, Humidity $37.2 \%$, Dew Point $49.9 \mathrm{~F}$ - Average Wind Velocity (if applicable) $.00 \mathrm{~m} / \mathrm{s}$, \# of physical barriers between sample area and outside (if applicable) 2

'Sample \#1

\begin{tabular}{|c|c|c|c|c|}
\hline \begin{tabular}{|l|} 
Bin \\
Range \\
(um)
\end{tabular} & \begin{tabular}{|l|} 
Bin Mean \\
Diameter \\
$(\mu \mathrm{m})$
\end{tabular} & $\begin{array}{l}\text { Diff. of the } \\
\log \text { of the } \\
\text { Size }\end{array}$ & Counts & $\begin{array}{l}\text { Weighted } \\
\text { Mean } \\
\text { Diameter }\end{array}$ \\
\hline $0.30-0.49$ & 0.38 & 0.21 & 35911 & \multirow{6}{*}{0.70} \\
\hline $0.50-0.99$ & 0.70 & 0.30 & 27136 & \\
\hline $1.00-1.99$ & 1.41 & 0.30 & 2964 & \\
\hline $2.00-4.99$ & 3.16 & 0.40 & 2348 & \\
\hline $5.00-9.99$ & 7.07 & 0.30 & 225 & \\
\hline$>10.00$ & 14.14 & 0.30 & 149 & \\
\hline \multicolumn{5}{|l|}{ Sample \#3 } \\
\hline \begin{tabular}{|l} 
Bin \\
Range \\
$(\mu \mathrm{m})$ \\
\end{tabular} & \begin{tabular}{|l} 
Bin Mean \\
Diameter \\
$(u m)$
\end{tabular} & $\begin{array}{l}\text { Diff. of the } \\
\log \text { of the } \\
\text { Size }\end{array}$ & Counts & $\begin{array}{l}\text { Weighted } \\
\text { Mean } \\
\text { Diameter }\end{array}$ \\
\hline $0.30-0.49$ & 0.38 & 0.21 & 14746 & \\
\hline $0.50-0.99$ & 0.70 & 0.30 & 21576 & \\
\hline $1.00-1.99$ & 1.41 & 0.30 & 8636 & \\
\hline $2.00-4.99$ & 3.16 & 0.40 & 19430 & \\
\hline $5.00-9.99$ & 7.07 & 0.30 & 4821 & \\
\hline$>10.00$ & 14.14 & 0.30 & 4347 & \\
\hline \multicolumn{5}{|l|}{ Sample \#5 } \\
\hline \begin{tabular}{|l} 
Bin \\
Range \\
$(\mu \mathrm{m})$ \\
\end{tabular} & \begin{tabular}{|l|} 
Bin Mean \\
Diameter \\
$(\omega \mathrm{m})$
\end{tabular} & \begin{tabular}{|l|} 
Diff. of the \\
log of the \\
Size
\end{tabular} & Counts & $\begin{array}{l}\text { Weighted } \\
\text { Mean } \\
\text { Diameter }\end{array}$ \\
\hline $0.30-0.49$ & 0.38 & 0.21 & 26525 & \multirow{6}{*}{1.38} \\
\hline $0.50-0.99$ & 0.70 & 0.30 & 23503 & \\
\hline $1.00-1.99$ & 1.41 & 0.30 & 4385 & \\
\hline $2.00-4.99$ & 3.16 & 0.40 & 6865 & \\
\hline $5.00-9.99$ & 7.07 & 0.30 & 1419 & \\
\hline$>10.00$ & 14.14 & 0.30 & 1722 & \\
\hline \multicolumn{5}{|l|}{ Sample \#7 } \\
\hline $\begin{array}{l}\text { Bin } \\
\text { Range } \\
(\text { (um) } \\
\end{array}$ & $\begin{array}{l}\text { Bin Mean } \\
\text { Diameter } \\
(\mu \mathrm{m})\end{array}$ & \begin{tabular}{|l|} 
Diff. of the \\
log of the \\
Size
\end{tabular} & Counts & \begin{tabular}{|l|} 
Weighted \\
Mean \\
Diameter
\end{tabular} \\
\hline $0.30 \cdot 0.49$ & 0.38 & 0.21 & 30790 & \multirow{6}{*}{1.00} \\
\hline $0.50-0.99$ & 0.70 & 0.30 & 24364 & \\
\hline $1.00-1.99$ & 1.41 & 0.30 & 3473 & \\
\hline $2.00-4.99$ & 3.16 & 0.40 & 4315 & \\
\hline $5.00-9.99$ & 7.07 & 0.30 & 797 & \\
\hline$>10.00$ & 14.14 & 0.30 & 802 & \\
\hline \multicolumn{5}{|l|}{ Sample \#9 } \\
\hline $\begin{array}{l}\text { Bin } \\
\text { Range } \\
(\mu \mathrm{m})\end{array}$ & \begin{tabular}{|l|} 
Bin Mean \\
Diameter \\
$(u m)$
\end{tabular} & \begin{tabular}{|l|} 
Diff. of the \\
log of the \\
Size
\end{tabular} & Counts & $\begin{array}{l}\text { Weighted } \\
\text { Mean } \\
\text { Diameter }\end{array}$ \\
\hline $0.30-0.49$ & 0.38 & $\begin{array}{l}0.21 \\
\end{array}$ & 32333 & \multirow{6}{*}{0.89} \\
\hline $0.50 \cdot 0.99$ & 0.70 & 0.30 & 24627 & \\
\hline $1.00-1.99$ & 1.41 & 0.30 & 3253 & \\
\hline $2.00-4.99$ & 3.16 & 0.40 & 3577 & \\
\hline $5.00-9.99$ & 7.07 & 0.30 & 561 & \\
\hline$>10.00$ & 14.14 & 0.30 & 595 & \\
\hline
\end{tabular}

Sample \#2

\begin{tabular}{|c|c|c|c|c|}
\hline $\begin{array}{l}\text { Bin } \\
\text { Range } \\
(\mu \mathrm{m})\end{array}$ & \begin{tabular}{|l|} 
Bin Mean \\
Diameter \\
$(\mathrm{l} / \mathrm{m})$
\end{tabular} & $\begin{array}{l}\text { Diff. of the } \\
\log \text { of the } \\
\text { Size }\end{array}$ & Counts & $\begin{array}{l}\text { Weighted } \\
\text { Mean } \\
\text { Diameter }\end{array}$ \\
\hline $0.30-0.49$ & .38 & 0.21 & 26546 & \multirow{6}{*}{1.81} \\
\hline $0.50-0.99$ & .70 & 0.30 & 24784 & \\
\hline $1.00-1.99$ & 1.41 & 0.30 & 5387 & \\
\hline $2.00-4.99$ & 3.16 & 0.40 & 9448 & \\
\hline $5.00-9.99$ & 7.07 & 0.30 & 2393 & \\
\hline$>10.00$ & 14.14 & 0.30 & 3418 & \\
\hline
\end{tabular}

Sample \#4

\begin{tabular}{|c|c|c|c|c|}
\hline $\begin{array}{l}\text { Bin } \\
\text { Range } \\
(\mu \mathrm{m}) \\
\end{array}$ & $\begin{array}{l}\text { Bin Mean } \\
\text { Diameter } \\
(\mu \mathrm{m})\end{array}$ & \begin{tabular}{|l|} 
Diff. of the \\
log of the \\
Size \\
\end{tabular} & Counts & \begin{tabular}{|l} 
Weighted \\
Mean \\
Diameter
\end{tabular} \\
\hline $0.30-0.49$ & .38 & 0.21 & 24397 & \multirow{6}{*}{1.63} \\
\hline $0.50-0.99$ & .70 & 0.30 & 23632 & \\
\hline $1.00-1.99$ & 1.41 & 0.30 & 5247 & \\
\hline $2.00-4.99$ & 3.16 & 0.40 & 9112 & \\
\hline $5.00-9.99$ & 7.07 & 0.30 & 1981 & \\
\hline$>10.00$ & $\overline{14.14}$ & 0.30 & 2332 & \\
\hline
\end{tabular}

\begin{tabular}{|c|c|c|c|c|}
\hline \multicolumn{5}{|l|}{ Sample \#6 } \\
\hline \begin{tabular}{|l} 
Bin \\
Range \\
$($ (um)
\end{tabular} & $\begin{array}{l}\text { Bin Mean } \\
\text { Diameter } \\
(u m)\end{array}$ & $\begin{array}{l}\text { Diff. of the } \\
\text { log of the } \\
\text { Size }\end{array}$ & Counts & $\begin{array}{l}\text { Weighted } \\
\text { Mean } \\
\text { Diameter }\end{array}$ \\
\hline $0.30-0.49$ & .38 & 0.21 & 30191 & \multirow{6}{*}{1.09} \\
\hline $0.50-0.99$ & .70 & 0.30 & 24604 & \\
\hline $1.00-1.99$ & 1.41 & 0.30 & 3773 & \\
\hline $2.00-4.99$ & 3.16 & 0.40 & 4823 & \\
\hline $5.00-9.99$ & 7.07 & 0.30 & 951 & \\
\hline$>10.00$ & 14.14 & 0.30 & 1092 & \\
\hline
\end{tabular}
Sample \#8

\begin{tabular}{|c|c|c|c|c|}
\hline $\begin{array}{l}\text { Bin } \\
\text { Range } \\
(\mathrm{um}) \\
\end{array}$ & \begin{tabular}{|l} 
Bin Mean \\
Diameter \\
$(u m)$
\end{tabular} & $\begin{array}{l}\text { Diff. of the } \\
\text { log of the } \\
\text { Size }\end{array}$ & Counts & $\begin{array}{l}\text { Woighted } \\
\text { Mean } \\
\text { Diameter }\end{array}$ \\
\hline $0.30-0.49$ & .38 & 0.21 & 31481 & \multirow{6}{*}{0.94} \\
\hline $0.50-0.99$ & .70 & 0.30 & 24605 & \\
\hline $0.00-1.99$ & 1.41 & 0.30 & 3529 & \\
\hline $2.00-4.99$ & 3.16 & 0.40 & 3902 & \\
\hline $5.00-9.99$ & 7.07 & 0.30 & 629 & \\
\hline$>10.00$ & 14.14 & 0.30 & 732 & \\
\hline
\end{tabular}

\begin{tabular}{|c|c|c|c|c|}
\hline \multicolumn{5}{|c|}{ Sample \#10 } \\
\hline $\begin{array}{l}\text { Bin } \\
\text { Range } \\
(\mathrm{rm})\end{array}$ & \begin{tabular}{|l|} 
Bin Mean \\
Diameter \\
$(u m)$
\end{tabular} & $\begin{array}{l}\text { Diff. of the } \\
\log \text { of the } \\
\text { Size }\end{array}$ & Counts & $\begin{array}{l}\text { Weighted } \\
\text { Mean } \\
\text { Diameter }\end{array}$ \\
\hline $0.30-0.49$ & .38 & 0.21 & 33075 & \multirow{6}{*}{0.84} \\
\hline $0.50-0.99$ & .70 & 0.30 & 25243 & \\
\hline $1.00-1.99$ & 1.41 & 0.30 & 3266 & \\
\hline $2.00-4.99$ & 3.16 & 0.40 & 3260 & \\
\hline $5.00-9.99$ & 7.07 & 0.30 & 537 & \\
\hline$>10.00$ & 14.14 & 0.30 & 446 & \\
\hline
\end{tabular}




\section{Aerosol Particle Measurement Record}

Description of sample location 3706 Building, Resuspension Test, at $1.5 \mathrm{~m}$, Date $8 / 26 / 94$, Time sample started 13:17:27, Temperature 76.9 F, Humidity 37.5\%, Dew Point 49.1 F - Average Wind Velocity (if applicable) $.00 \mathrm{~m} / \mathrm{s}$, \# of physical barriers between sample area and outside (if applicable) 2

\begin{tabular}{|c|c|c|c|c|}
\hline $\begin{array}{l}\text { Bin } \\
\text { Range } \\
\text { (um) }\end{array}$ & $\begin{array}{l}\text { Bin Mean } \\
\text { Diameter } \\
(\mu \mathrm{m})\end{array}$ & $\begin{array}{l}\text { Diff. of the } \\
\log \text { of the } \\
\text { Size }\end{array}$ & Counts & $\begin{array}{l}\text { Weighted } \\
\text { Mean } \\
\text { Diameter }\end{array}$ \\
\hline $0,30 \cdot 0.49$ & 0.38 & 0.21 & 32880 & \multirow{6}{*}{0.73} \\
\hline $0.50-0.99$ & 0.70 & 0.30 & 9616 & \\
\hline $1.00-1.99$ & 1.41 & 0.30 & 2490 & \\
\hline $2.00-4.99$ & 3.16 & 0.40 & 2256 & \\
\hline $5.00-9.99$ & 7.07 & 0.30 & 233 & \\
\hline$>10.00$ & 14.14 & 0.30 & 236 & \\
\hline \multicolumn{5}{|l|}{ Sample \#3 } \\
\hline $\begin{array}{l}\text { Bin } \\
\text { Range } \\
(\mu \mathrm{m}) \\
\end{array}$ & \begin{tabular}{|l} 
Bin Mean \\
Diameter \\
$(\mu \mathrm{m})$
\end{tabular} & \begin{tabular}{|l} 
Diff. of the \\
log of the \\
Size
\end{tabular} & Counts & $\begin{array}{l}\text { Weighted } \\
\text { Mean } \\
\text { Diameter }\end{array}$ \\
\hline $0.30-0.49$ & 0.38 & 0.21 & 27125 & \multirow{6}{*}{1.29} \\
\hline $0.50-0.99$ & 0.70 & 0.30 & 10238 & \\
\hline $1.00-1.99$ & 1.41 & 0.30 & 3813 & \\
\hline $2.00 \cdot 4.99$ & 3.16 & 0.40 & 5919 & \\
\hline $5.00-9.99$ & 7.07 & 0.30 & 1188 & \\
\hline$>10.00$ & 14.14 & 0.30 & 941 & \\
\hline \multicolumn{5}{|l|}{ Sample $\# 5$} \\
\hline \begin{tabular}{|l|} 
Bin \\
Range \\
$(\mu \mathrm{m})$ \\
\end{tabular} & \begin{tabular}{|l}
$\begin{array}{l}\text { Bin Mean } \\
\text { Diameter } \\
(\mu \mathrm{m})\end{array}$ \\
\end{tabular} & $\begin{array}{l}\text { Diff. of the } \\
\text { log of the } \\
\text { Size }\end{array}$ & Counts & \begin{tabular}{|l|} 
Weighted \\
Mean \\
Diameter
\end{tabular} \\
\hline $0.30-0.49$ & 0.38 & 0.21 & 23393 & \multirow{6}{*}{1.72} \\
\hline $0.50-0.99$ & 0.70 & 0.30 & 10968 & \\
\hline $1.00-1.99$ & 1.41 & 0.30 & 4930 & \\
\hline $2.00-4.99$ & 3.16 & 0.40 & 9647 & \\
\hline $5.00-9.99$ & 7.07 & 0.30 & 2137 & \\
\hline$>10.00$ & 14.14 & 0.30 & 1503 & \\
\hline \multicolumn{5}{|l|}{ Sample \#7 } \\
\hline \begin{tabular}{|l} 
Bin \\
Range \\
(um)
\end{tabular} & \begin{tabular}{|l} 
Bin Mean \\
Diameter \\
$(u m)$
\end{tabular} & $\begin{array}{l}\text { Diff. of the } \\
\text { log of the } \\
\text { Size }\end{array}$ & Counts & \begin{tabular}{|l|} 
Weighted \\
Mean \\
Diameter
\end{tabular} \\
\hline $0.30-0.49$ & 0.38 & 0.21 & 27783 & \multirow{6}{*}{1.27} \\
\hline $0.50-0.99$ & 0.70 & 0.30 & 10412 & \\
\hline $1.00-1.99$ & 1.41 & 0.30 & 3787 & \\
\hline $2.00-4.99$ & 3.16 & 0.40 & 6227 & \\
\hline $5.00-9.99$ & 7.07 & 0.30 & 1197 & \\
\hline$>10.00$ & 14.14 & 0.30 & 872 & \\
\hline \multicolumn{5}{|l|}{ Sample \#9 } \\
\hline \begin{tabular}{|l|} 
Bin \\
Range \\
(um) \\
\end{tabular} & \begin{tabular}{|l|} 
Bin Mean \\
Diameter \\
$(\mu \mathrm{m})$
\end{tabular} & \begin{tabular}{|l|} 
Diff. of the \\
log of the \\
Size
\end{tabular} & Counts & \begin{tabular}{|l|} 
Weighted \\
Mean \\
Diameter
\end{tabular} \\
\hline $0.30-0.49$ & 0.38 & 0.21 & 30299 & \multirow{6}{*}{1.00} \\
\hline $0.50-0.99$ & 0.70 & 0.30 & 10070 & \\
\hline $1.00-1.99$ & 1.41 & 0.30 & 3350 & \\
\hline $2.00-4.99$ & 3.16 & 0.40 & 4475 & \\
\hline $5.00-9.99$ & 7.07 & 0.30 & 732 & \\
\hline$>10.00$ & 14.14 & 0.30 & 493 & \\
\hline
\end{tabular}

\begin{tabular}{|c|c|c|c|c|}
\hline $\begin{array}{l}\text { Bin } \\
\text { Range } \\
\text { (um) }\end{array}$ & $\begin{array}{l}\text { Bin Mean } \\
\text { Diameter } \\
(u m)\end{array}$ & $\begin{array}{l}\text { Diff. of the } \\
\log \text { of the } \\
\text { Size }\end{array}$ & Counts & $\begin{array}{l}\text { Weighted } \\
\text { Mean } \\
\text { Diameter }\end{array}$ \\
\hline $0.30-0.49$ & .38 & 0.21 & 33082 & \multirow{6}{*}{0.69} \\
\hline $0.50-0.99$ & .70 & 0.30 & 9376 & \\
\hline $1.00-1.99$ & 1.41 & 0.30 & 2366 & \\
\hline $2.00-4.99$ & 3.16 & 0.40 & 2184 & \\
\hline $5.00-9.99$ & 7.07 & 0.30 & 198 & \\
\hline$>10.00$ & 14.14 & 0.30 & 141 & \\
\hline \multicolumn{5}{|l|}{ Sample \#4 } \\
\hline \begin{tabular}{|l|} 
Bin \\
Range \\
$(\mu \mathrm{m})$ \\
\end{tabular} & \begin{tabular}{|l} 
Bin Mean \\
Diameter \\
(um)
\end{tabular} & $\begin{array}{l}\text { Diff. of the } \\
\text { log of the } \\
\text { Size }\end{array}$ & Counts & $\begin{array}{l}\text { Weighted } \\
\text { Mean } \\
\text { Diameter }\end{array}$ \\
\hline $0.30-0.49$ & .38 & 0.21 & 21662 & \\
\hline $0.50-0.99$ & .70 & 0.30 & 11172 & \\
\hline $1.00-1.99$ & 1.41 & 0.30 & 5521 & \\
\hline $2.00-4.99$ & 3.16 & 0.40 & 11529 & \\
\hline $5.00-9.99$ & 7.07 & 0.30 & 2654 & \\
\hline$>10.00$ & 14.14 & 0.30 & 1857 & \\
\hline \multicolumn{5}{|c|}{ Sample \#6 } \\
\hline $\begin{array}{l}\text { Bin } \\
\text { Range } \\
(\mu \mathrm{m})\end{array}$ & $\begin{array}{l}\text { Bin Mean } \\
\text { Diameter } \\
(\mu \mathrm{m})\end{array}$ & \begin{tabular}{|l|} 
Diff. of the \\
log of the \\
Size
\end{tabular} & Counts & \begin{tabular}{|l|}
$\begin{array}{l}\text { Weighted } \\
\text { Mean } \\
\text { Diameter }\end{array}$ \\
\end{tabular} \\
\hline $0.30-0.49$ & .38 & 0.21 & 25756 & \multirow{6}{*}{1.44} \\
\hline $0.50-0.99$ & .70 & 0.30 & 10429 & \\
\hline $1.00-1.99$ & 1.41 & 0.30 & 4171 & \\
\hline $2.00-4.99$ & 3.16 & 0.40 & 7664 & \\
\hline $5.00-9.99$ & 7.07 & 0.30 & 1551 & \\
\hline$>10.00$ & 14.14 & 0.30 & 1055 & \\
\hline \multicolumn{5}{|l|}{ Sample \#8 } \\
\hline $\begin{array}{l}\text { Bin } \\
\text { Range } \\
(u m) \\
\end{array}$ & \begin{tabular}{|l} 
Bin Mean \\
Diameter \\
$(\mu \mathrm{m})$
\end{tabular} & $\begin{array}{l}\text { Diff. of the } \\
\text { log of the } \\
\text { Size }\end{array}$ & Counts & $\begin{array}{l}\text { Weighted } \\
\text { Mean } \\
\text { Diametor }\end{array}$ \\
\hline $0.30-0.49$ & .38 & 0.21 & 28848 & \multirow{6}{*}{1.14} \\
\hline $0.50-0.99$ & .70 & 0.30 & 10299 & \\
\hline $0.00-1.99$ & 1.41 & 0.30 & 3629 & \\
\hline $2.00-4.99$ & 3.16 & 0.40 & 5448 & \\
\hline $5.00-9.99$ & 7.07 & 0.30 & 999 & \\
\hline$>10.00$ & 14.14 & 0.30 & 656 & \\
\hline \multicolumn{5}{|c|}{ Sample $\# 10$} \\
\hline $\begin{array}{l}\text { Bin } \\
\text { Range } \\
(\mu \mathrm{m})\end{array}$ & $\begin{array}{l}\text { Bin Mean } \\
\text { Diameter } \\
(\mu \mathrm{m})\end{array}$ & \begin{tabular}{|l|} 
Diff. of the \\
log of the \\
Size
\end{tabular} & Counts & $\begin{array}{l}\text { Weighted } \\
\text { Mean } \\
\text { Diamoter }\end{array}$ \\
\hline $0.30-0.49$ & .38 & 0.21 & 31044 & \multirow{6}{*}{0.93} \\
\hline $0.50-0.99$ & .70 & 0.30 & 10178 & \\
\hline $1.00-1.99$ & 1.41 & 0.30 & 3123 & \\
\hline $2.00-4.99$ & 3.16 & 0.40 & 3913 & \\
\hline $5.00-9.99$ & 7.07 & 0.30 & 613 & \\
\hline$>10.00$ & 14.14 & 0.30 & 421 & \\
\hline
\end{tabular}




\section{Aerosol Particle Measurement Record}

Description of sample location 3706 Building, Resuspension Test, at .25 m, Date 8/26/94, Time sample started 13:17:27, Temperature 76.9 F, Humidity 37.5\%, Dew Point 49.1 F , Average Wind Velocity (if applicable) $.00 \mathrm{~m} / \mathrm{s}$, \# of physical barriers between sample area and outside (if applicable) 2

Sample \#1

\begin{tabular}{|c|c|c|c|c|}
\hline $\begin{array}{l}\text { Bin } \\
\text { Range } \\
(\mathrm{sm})\end{array}$ & \begin{tabular}{|l} 
Bin Mean \\
Diameter \\
(um)
\end{tabular} & $\begin{array}{l}\text { Diff. of the } \\
\text { log of the } \\
\text { Size }\end{array}$ & Counts & \begin{tabular}{|l|} 
Weighted \\
Mean \\
Diameter
\end{tabular} \\
\hline $0.30-0.49$ & 0.38 & 0.21 & 32698 & \multirow{6}{*}{0.72} \\
\hline $0.50-0.99$ & 0.70 & 0.30 & 20745 & \\
\hline $1.00-1.99$ & 1.41 & 0.30 & 2287 & \\
\hline $2.00-4.99$ & 3.16 & 0.40 & 2191 & \\
\hline $5.00-9.99$ & 7.07 & 0.30 & 198 & \\
\hline$>10.00$ & 14.14 & 0.30 & 237 & \\
\hline \multicolumn{5}{|l|}{ Sample $\# 3$} \\
\hline $\begin{array}{l}\text { Bin } \\
\text { Range } \\
(u m)\end{array}$ & \begin{tabular}{|l|} 
Bin Mean \\
Diameter \\
$(\mu \mathrm{m})$
\end{tabular} & \begin{tabular}{|l|} 
Diff. of the \\
log of the \\
Size
\end{tabular} & Counts & \begin{tabular}{|l|} 
Weighted \\
Mean \\
Diameter
\end{tabular} \\
\hline $0.30-0.49$ & 0.38 & 0.21 & 18867 & \multirow{6}{*}{2.05} \\
\hline $0.50 \cdot 0.99$ & 0.70 & 0.30 & 18845 & \\
\hline $1.00-1.99$ & 1.41 & 0.30 & 5322 & \\
\hline $2.00-4.99$ & 3.16 & 0.40 & 11021 & \\
\hline $5.00-9.99$ & 7.07 & 0.30 & 2652 & \\
\hline$>10.00$ & 14.14 & 0.30 & 2887 & \\
\hline \multicolumn{5}{|l|}{ Sample \#5 } \\
\hline $\begin{array}{l}\text { Bin } \\
\text { Range } \\
(\mu \mathrm{m}) \\
\end{array}$ & \begin{tabular}{|l} 
Bin Mean \\
Diameter \\
$(\mu \mathrm{m})$
\end{tabular} & $\begin{array}{l}\text { Diff. of the } \\
\log \text { of the } \\
\text { Size }\end{array}$ & Counts & $\begin{array}{l}\text { Weighted } \\
\text { Mean } \\
\text { Diameter }\end{array}$ \\
\hline $0.30-0.49$ & 0.38 & 0.21 & 24493 & \multirow{6}{*}{1.41} \\
\hline $0.50-0.99$ & 0.70 & 0.30 & 19368 & \\
\hline $1.00-1.99$ & 1.41 & 0.30 & 3900 & \\
\hline $2.00-4.99$ & 3.16 & 0.40 & 6851 & \\
\hline $5,00-9.99$ & 7.07 & 0.30 & 1537 & \\
\hline$>10.00$ & 14.14 & 0.30 & 1456 & \\
\hline
\end{tabular}

\begin{tabular}{|c|c|c|c|c|}
\hline \multicolumn{5}{|l|}{ Sample \#7 } \\
\hline $\begin{array}{l}\text { Bin } \\
\text { Range } \\
(\omega \mathrm{m})\end{array}$ & $\begin{array}{l}\text { Bin Mean } \\
\text { Diameter } \\
(\mathrm{um})\end{array}$ & $\begin{array}{l}\text { Diff. of the } \\
\log \text { of the } \\
\text { Size }\end{array}$ & Counts & $\begin{array}{l}\text { Weighted } \\
\text { Mean } \\
\text { Diameter }\end{array}$ \\
\hline $0.30 \cdot 0.49$ & 0.38 & 0.21 & 28569 & \multirow{6}{*}{1.07} \\
\hline $0.50-0.99$ & 0.70 & 0.30 & 20601 & \\
\hline $1.00-1.99$ & 1.41 & 0.30 & 3231 & \\
\hline $2.00 \cdot 4.99$ & 3.16 & 0.40 & 4638 & \\
\hline $5,00-9.99$ & 7.07 & 0.30 & 874 & \\
\hline$>10.00$ & 14.14 & 0.30 & 857 & \\
\hline \multicolumn{5}{|l|}{ Sample \#9 } \\
\hline \begin{tabular}{|l|} 
Bin \\
Range \\
$(\mu \mathrm{m})$
\end{tabular} & \begin{tabular}{|l|} 
Bin Mean \\
Diameter \\
$(\mu \mathrm{m})$
\end{tabular} & $\begin{array}{l}\text { Diff. of the } \\
\log \text { of the } \\
\text { Size }\end{array}$ & Counts & $\begin{array}{l}\text { Weighted } \\
\text { Mean } \\
\text { Diameter }\end{array}$ \\
\hline $0.30 \cdot 0.49$ & 0.38 & 0.21 & 32213 & \multirow{6}{*}{0.91} \\
\hline $0.50-0.99$ & 0.70 & 0.30 & 21503 & \\
\hline $1.00-1.99$ & 1.41 & 0.30 & 2879 & \\
\hline $2.00-4.99$ & 3.16 & 0.40 & 3681 & \\
\hline $5.00-9.99$ & 7.07 & 0.30 & 633 & \\
\hline$>10.00$ & 14.14 & 0.30 & 606 & \\
\hline
\end{tabular}

Sample \#2

\begin{tabular}{|c|c|c|c|c|}
\hline $\begin{array}{l}\text { Bin } \\
\text { Range } \\
\text { (um) }\end{array}$ & $\begin{array}{l}\text { Bin Mean } \\
\text { Diameter } \\
\text { (um) }\end{array}$ & $\begin{array}{l}\text { Diff. of the } \\
\text { log of the } \\
\text { Size }\end{array}$ & Counts & $\begin{array}{l}\text { Weighted } \\
\text { Mean } \\
\text { Diameter }\end{array}$ \\
\hline $0.30-0.49$ & .38 & 0.21 & 18059 & \\
\hline $0.50-0.99$ & .70 & 0.30 & 19076 & \\
\hline $1.00-1.99$ & 1.41 & 0.30 & 6928 & \\
\hline $2.00-4.99$ & 3.16 & 0.40 & 18895 & \\
\hline $5.00-9.99$ & 7.07 & 0.30 & 7216 & \\
\hline$>10.00$ & 14.14 & 0.30 & 15452 & \\
\hline \multicolumn{5}{|l|}{ Sample \#4 } \\
\hline \begin{tabular}{|l|} 
Bin \\
Range \\
(um) \\
\end{tabular} & $\begin{array}{l}\text { Bin Mean } \\
\text { Diameter } \\
(\text { (um) }\end{array}$ & $\begin{array}{l}\text { Diff. of the } \\
\text { log of the } \\
\text { Size }\end{array}$ & Counts & $\begin{array}{l}\text { Weighted } \\
\text { Mean } \\
\text { Diameter }\end{array}$ \\
\hline $0.30-0.49$ & .38 & 0.21 & 19424 & \multirow{6}{*}{2.01} \\
\hline $0.50-0.99$ & .70 & 0.30 & 18847 & \\
\hline $1.00-1.99$ & 1.41 & 0.30 & $\overline{5239}$ & \\
\hline $2.00-4.99$ & 3.16 & 0.40 & 10587 & \\
\hline $5.00-9.99$ & 7.07 & 0.30 & 2683 & \\
\hline$>10.00$ & 14.14 & 0.30 & 2792 & \\
\hline
\end{tabular}

\begin{tabular}{|c|c|c|c|c|}
\hline $\begin{array}{l}\text { Bin } \\
\text { Range } \\
\text { (um) }\end{array}$ & $\begin{array}{l}\text { Bin Mean } \\
\text { Diameter } \\
(\mathrm{em})\end{array}$ & $\begin{array}{l}\text { Diff. of the } \\
\text { log of the } \\
\text { Size }\end{array}$ & Counts & $\begin{array}{l}\text { Weighted } \\
\text { Mean } \\
\text { Diameter }\end{array}$ \\
\hline $0.30-0.49$ & .38 & 0.21 & 26593 & \multirow{6}{*}{1.25} \\
\hline $0.50-0.99$ & .70 & 0.30 & 19426 & \\
\hline $1.00-1.99$ & 1.41 & 0.30 & 3391 & \\
\hline $2.00-4.99$ & 3.16 & 0.40 & 5515 & \\
\hline $5.00-9.99$ & 7.07 & 0.30 & 1134 & \\
\hline$>10.00$ & 14.14 & 0.30 & 1265 & \\
\hline
\end{tabular}

\begin{tabular}{|c|c|c|c|c|}
\hline \multicolumn{5}{|l|}{ Sample \#8 } \\
\hline $\begin{array}{l}\text { Bin } \\
\text { Range } \\
\text { (um) }\end{array}$ & \begin{tabular}{|l} 
Bin Mean \\
Diameter \\
$(\mu \mathrm{m})$
\end{tabular} & $\begin{array}{l}\text { Diff. of the } \\
\text { log of the } \\
\text { Size }\end{array}$ & Counts & $\begin{array}{l}\text { Weighted } \\
\text { Mean } \\
\text { Diamoter }\end{array}$ \\
\hline $0.30-0.49$ & .38 & 0.21 & 29580 & \multirow{6}{*}{0.99} \\
\hline $0.50-0.99$ & .70 & 0.30 & 20622 & \\
\hline $0.00-1.99$ & 1.41 & 0.30 & $\overline{3018}$ & \\
\hline $2.00-4.99$ & 3.16 & 0.40 & 4081 & \\
\hline $5.00-9.99$ & 7.07 & 0.30 & 745 & \\
\hline$>10.00$ & 14.14 & 0.30 & 705 & \\
\hline
\end{tabular}

\begin{tabular}{|c|c|c|c|c|}
\hline \multicolumn{5}{|c|}{ Sample $\# 10$} \\
\hline $\begin{array}{l}\text { Bin } \\
\text { Range } \\
(\mu \mathrm{m})\end{array}$ & \begin{tabular}{|l|} 
Bin Mean \\
Diameter \\
$(\mu \mathrm{m})$
\end{tabular} & $\begin{array}{l}\text { Diff. of the } \\
\text { log of the } \\
\text { Size }\end{array}$ & Counts & $\begin{array}{l}\text { Weighted } \\
\text { Mean } \\
\text { Diameter }\end{array}$ \\
\hline $0.30-0.49$ & .38 & 0.21 & 31174 & \multirow{6}{*}{0.90} \\
\hline $0.50-0.99$ & .70 & 0.30 & 21770 & \\
\hline $1.00-1.99$ & 1.41 & 0.30 & 2935 & \\
\hline $2.00-4.99$ & 3.16 & 0.40 & 3652 & \\
\hline $5.00-9.99$ & 7.07 & 0.30 & 599 & \\
\hline$>10.00$ & 14.14 & 0.30 & 552 & \\
\hline
\end{tabular}




\section{Aerosol Particle Measurement Record}

Description of sample location 3706 Building, Resuspension Test, at $2.0 \mathrm{~m}$, Date $8 / 26 / 94$, Time sample started 12:34:27, Temperature_76.7 F, Humidity 37.9\%, Dew Point 48.9 F , Average Wind Velocity (if applicable) $.00 \mathrm{~m} / \mathrm{s}$, \# of physical barriers between sample area and outside (if applicable) $\underline{2}$

\begin{tabular}{|c|c|c|c|c|}
\hline \begin{tabular}{|l} 
Bin \\
Range \\
$(\mu \mathrm{m})$
\end{tabular} & $\begin{array}{l}\text { Bin Mean } \\
\text { Diameter } \\
(u m)\end{array}$ & $\begin{array}{l}\text { Diff. of the } \\
\text { log of the } \\
\text { Size }\end{array}$ & Counts & \begin{tabular}{|l} 
Weighted \\
Mean \\
Diameter
\end{tabular} \\
\hline $0.30-0.49$ & 0.38 & 0.21 & 31919 & \multirow{6}{*}{0.62} \\
\hline $0.50-0.99$ & 0.70 & 0.30 & 8573 & \\
\hline $1.00-1.99$ & 1.41 & 0.30 & 1881 & \\
\hline $2.00-4.99$ & 3.16 & 0.40 & 1473 & \\
\hline $5.00-9.99$ & 7.07 & 0.30 & 118 & \\
\hline$>10.00$ & 14.14 & 0.30 & 83 & \\
\hline \multicolumn{5}{|l|}{ Sample \#3 } \\
\hline \begin{tabular}{|l} 
Bin \\
Range \\
(um) \\
\end{tabular} & $\begin{array}{l}\text { Bin Mean } \\
\text { Diameter } \\
(\mu \mathrm{m})\end{array}$ & $\begin{array}{l}\text { Diff. of the } \\
\log \text { of the } \\
\text { Size }\end{array}$ & Counts & $\begin{array}{l}\text { Weighted } \\
\text { Mean } \\
\text { Diameter }\end{array}$ \\
\hline $0.30-0.49$ & 0.38 & 0.21 & 24250 & \multirow{6}{*}{1.57} \\
\hline $0.50-0.99$ & 0.70 & 0.30 & 10086 & \\
\hline $1.00-1.99$ & 1.41 & 0.30 & 4017 & \\
\hline $2.00-4.99$ & 3.16 & 0.40 & 7715 & \\
\hline $5.00-9.99$ & 7.07 & 0.30 & 1709 & \\
\hline$>10.00$ & 14.14 & 0.30 & 1313 & \\
\hline \multicolumn{5}{|l|}{ Sample \#5 } \\
\hline \begin{tabular}{|l} 
Bin \\
Range \\
$(\mu \mathrm{m})$ \\
\end{tabular} & $\begin{array}{l}\text { Bin Mean } \\
\text { Diameter } \\
(\text { (em) }\end{array}$ & $\begin{array}{l}\text { Diff. of the } \\
\log \text { of the } \\
\text { Size }\end{array}$ & Counts & $\begin{array}{l}\text { Weighted } \\
\text { Mean } \\
\text { Diameter }\end{array}$ \\
\hline $0.30-0.49$ & 0.38 & 0.21 & 17755 & \multirow{6}{*}{2.35} \\
\hline $0.50-0.99$ & 0.70 & 0.30 & 10663 & \\
\hline $1.00-1.99$ & 1.41 & 0.30 & 5957 & \\
\hline $2.00 \cdot 4.99$ & 3.16 & 0.40 & 13784 & \\
\hline $5.00-9.99$ & 7.07 & 0.30 & 3578 & \\
\hline$>10.00$ & 14.14 & 0.30 & 2548 & \\
\hline \multicolumn{5}{|l|}{ Sample \#7 } \\
\hline $\begin{array}{l}\text { Bin } \\
\text { Range } \\
(\mu \mathrm{m})\end{array}$ & $\begin{array}{l}\text { Bin Mean } \\
\text { Diameter } \\
(\mu \mathrm{m})\end{array}$ & \begin{tabular}{|l|} 
Diff. of the \\
log of the \\
Size
\end{tabular} & Counts & $\begin{array}{l}\text { Weighted } \\
\text { Mean } \\
\text { Diameter }\end{array}$ \\
\hline $0.30 \cdot 0.49$ & $\begin{array}{l}0.38 \\
\end{array}$ & 0.21 & 23185 & \multirow{6}{*}{1.68} \\
\hline $0.50-0.99$ & 0.70 & 0.30 & 9760 & \\
\hline $1.00-1.99$ & 1.41 & 0.30 & 4263 & \\
\hline $2.00-4.99$ & 3.16 & 0.40 & 8438 & \\
\hline $5.00-9.99$ & 7.07 & 0.30 & 2002 & \\
\hline$>10.00$ & 14.14 & 0.30 & 1391 & \\
\hline \multicolumn{5}{|l|}{ Sample \#9 } \\
\hline $\begin{array}{l}\text { Bin } \\
\text { Range } \\
(\mu \mathrm{m})\end{array}$ & $\begin{array}{l}\text { Bin Mean } \\
\text { Diameter } \\
(\mu \mathrm{m})\end{array}$ & $\begin{array}{l}\text { Diff. of the } \\
\text { log of the } \\
\text { Size }\end{array}$ & Counts & $\begin{array}{l}\text { Weighted } \\
\text { Mean } \\
\text { Diameter }\end{array}$ \\
\hline $0.30-0.49$ & 0.38 & 0.21 & 26932 & \multirow{6}{*}{1.24} \\
\hline $0.50-0.99$ & 0.70 & 0.30 & 9392 & \\
\hline $1.00-1.99$ & 1.41 & 0.30 & 3137 & \\
\hline $2.00-4.99$ & 3.16 & 0.40 & 5126 & \\
\hline $5.00-9.99$ & 7.07 & 0.30 & 1159 & \\
\hline$>10.00$ & 14.14 & 0.30 & 862 & \\
\hline
\end{tabular}

\begin{tabular}{|c|c|c|c|c|}
\hline \begin{tabular}{|l} 
Bin \\
Range \\
(fm)
\end{tabular} & $\begin{array}{l}\text { Bin Mean } \\
\text { Diameter } \\
(\mathrm{m})\end{array}$ & $\begin{array}{l}\text { Diff. of the } \\
\text { log of the } \\
\text { Size }\end{array}$ & Counts & $\begin{array}{l}\text { Weighted } \\
\text { Mean } \\
\text { Diameter }\end{array}$ \\
\hline $0.30-0.49$ & .38 & 0.21 & 32559 & \multirow{6}{*}{0.62} \\
\hline $0.50-0.99$ & .70 & 0.30 & 8587 & \\
\hline $1.00-1.99$ & 1.41 & 0.30 & 1944 & \\
\hline $2.00-4.99$ & 3.16 & 0.40 & 1500 & \\
\hline $5.00-9.99$ & 7.07 & 0.30 & 117 & \\
\hline$>10.00$ & 14.14 & 0.30 & 64 & \\
\hline \multicolumn{5}{|c|}{ Sample \#4 } \\
\hline $\begin{array}{l}\text { Bin } \\
\text { Range } \\
(u \mathrm{~mm})\end{array}$ & $\begin{array}{l}\text { Bin Mean } \\
\text { Diameter } \\
(\text { um) }\end{array}$ & $\begin{array}{l}\text { Diff. of the } \\
\text { log of the } \\
\text { Size }\end{array}$ & Counts & $\begin{array}{l}\text { Woighted } \\
\text { Mean } \\
\text { Diameter }\end{array}$ \\
\hline $0.30-0.49$ & .38 & 0.21 & 17567 & \\
\hline $0.50-0.99$ & .70 & 0.30 & 11200 & \\
\hline $1.00-1.99$ & 1.41 & 0.30 & 6224 & \\
\hline $2.00-4.99$ & 3.16 & 0.40 & 14828 & \\
\hline $5.00-9.99$ & 7.07 & 0.30 & 3760 & \\
\hline$>10.00$ & 14.14 & 0.30 & 2848 & \\
\hline
\end{tabular}

\begin{tabular}{|c|c|c|c|c|}
\hline \multicolumn{5}{|c|}{ Sample \#6 } \\
\hline $\begin{array}{l}\text { Bin } \\
\text { Range } \\
\text { (um) }\end{array}$ & $\begin{array}{l}\text { Bin Mean } \\
\text { Diameter } \\
(\mathrm{lm})\end{array}$ & $\begin{array}{l}\text { Diff. of the } \\
\log \text { of the } \\
\text { Size }\end{array}$ & Counts & $\begin{array}{l}\text { Woighted } \\
\text { Moan } \\
\text { Diameter }\end{array}$ \\
\hline $0.30-0.49$ & .38 & 0.21 & 20777 & \multirow{6}{*}{1.98} \\
\hline $0.50-0.99$ & .70 & 0.30 & 10112 & \\
\hline $1.00-1.99$ & 1.41 & 0.30 & 4947 & \\
\hline $2.00-4.99$ & 3.16 & 0.40 & 10569 & \\
\hline $5.00-9.99$ & 7.07 & 0.30 & 2648 & \\
\hline$>10.00$ & $\overline{14.14}$ & 0.30 & 1915 & \\
\hline
\end{tabular}

\begin{tabular}{|c|c|c|c|c|}
\hline \multicolumn{5}{|l|}{ Sample \#8 } \\
\hline $\begin{array}{l}\text { Bin } \\
\text { Range } \\
\text { (um) }\end{array}$ & $\begin{array}{l}\text { Bin Mean } \\
\text { Diameter } \\
(\mu \mathrm{m})\end{array}$ & $\begin{array}{l}\text { Diff. of the } \\
\log \text { of the } \\
\text { Size }\end{array}$ & Counts & $\begin{array}{l}\text { Woighted } \\
\text { Mean } \\
\text { Diameter }\end{array}$ \\
\hline $0.30-0.49$ & .38 & 0.21 & 25685 & \multirow{6}{*}{1.40} \\
\hline $0.50-0.99$ & .70 & 0.30 & 9426 & \\
\hline $0.00-1.99$ & 1.41 & 0.30 & 3540 & \\
\hline $2.00-4.99$ & 3.16 & 0.40 & 6241 & \\
\hline $5.00-9.99$ & 7.07 & 0.30 & 1510 & \\
\hline$>10.00$ & 14.14 & 0.30 & 1055 & \\
\hline
\end{tabular}

\begin{tabular}{|c|c|c|c|c|}
\hline \multicolumn{5}{|c|}{ Sample \#10 } \\
\hline $\begin{array}{l}\text { Bin } \\
\text { Range } \\
(u m)\end{array}$ & \begin{tabular}{|l|} 
Bin Mean \\
Diameter \\
$(\omega \mathrm{m})$
\end{tabular} & $\begin{array}{l}\text { Diff. of the } \\
\text { log of the } \\
\text { Size }\end{array}$ & Counts & $\begin{array}{l}\text { Woighted } \\
\text { Mean } \\
\text { Diamoter }\end{array}$ \\
\hline $0.30-0.49$ & .38 & 0.21 & 27530 & \multirow{6}{*}{1.13} \\
\hline $0.50-0.99$ & .70 & 0.30 & 9126 & \\
\hline $1.00-1.99$ & $\overline{1.41}$ & 0.30 & 2835 & \\
\hline $2.00-4.99$ & 3.16 & 0.40 & 4474 & \\
\hline $5.00-9.99$ & 7.07 & 0.30 & 967 & \\
\hline$>10.00$ & $\overline{14.14}$ & 0.30 & 693 & \\
\hline
\end{tabular}




\section{Aerosol Particle Measurement Record}

Description of sample location 3706 Building, Resuspension Test, at .25 m, Date 8/26/94, Time sample started 12:34:27, Temperature_76.7 F, Humidity 37.9 \%, Dew Point 48.9 F - Average Wind Velocity (if applicable) $.00 \mathrm{~m} / \mathrm{s}$, \# of physical barriers between sample area and outside (if applicable) 2

\begin{tabular}{|c|c|c|c|c|}
\hline $\begin{array}{l}\text { Bin } \\
\text { Range } \\
\text { (um) }\end{array}$ & \begin{tabular}{|l|} 
Bin Mean \\
Diameter \\
$($ (um)
\end{tabular} & $\begin{array}{l}\text { Diff. of the } \\
\log \text { of the } \\
\text { Size }\end{array}$ & Counts & $\begin{array}{l}\text { Weighted } \\
\text { Mean } \\
\text { Diameter }\end{array}$ \\
\hline $0.30-0.49$ & 0.38 & 0.21 & 31705 & \multirow{6}{*}{0.68} \\
\hline $0.50-0.99$ & 0.70 & 0.30 & 19465 & \\
\hline $1.00-1.99$ & 1.41 & 0.30 & 1949 & \\
\hline $2.00-4.99$ & 3.16 & 0.40 & 1706 & \\
\hline $5.00-9.99$ & 7.07 & 0.30 & 188 & \\
\hline$>10.00$ & 14.14 & 0.30 & 177 & \\
\hline \multicolumn{5}{|l|}{ Sample \#3 } \\
\hline $\begin{array}{l}\text { Bin } \\
\text { Range } \\
(\mu \mathrm{m})\end{array}$ & \begin{tabular}{|l} 
Bin Mean \\
Diameter \\
$(\mu \mathrm{m})$
\end{tabular} & $\begin{array}{l}\text { Diff. of the } \\
\text { log of the } \\
\text { Size }\end{array}$ & Counts & $\begin{array}{l}\text { Weighted } \\
\text { Mean } \\
\text { Diameter }\end{array}$ \\
\hline $0.30 \cdot 0.49$ & 0.38 & 0.21 & 11743 & \\
\hline $0.50 \cdot 0.99$ & 0.70 & 0.30 & 16436 & \\
\hline $1.00-1.99$ & 1.41 & 0.30 & 7272 & \\
\hline $2.00-4.99$ & 3.16 & 0.40 & 19200 & \\
\hline $5.00-9.99$ & 7.07 & 0.30 & 5951 & \\
\hline$>10.00$ & 14.14 & 0.30 & 5815 & \\
\hline \multicolumn{5}{|l|}{ Sample \#5 } \\
\hline \begin{tabular}{|l} 
Bin \\
Range \\
$(\mu \mathrm{m})$ \\
\end{tabular} & \begin{tabular}{|l} 
Bin Mean \\
Diameter \\
$(u m)$
\end{tabular} & $\begin{array}{l}\text { Diff. of the } \\
\text { log of the } \\
\text { Size }\end{array}$ & Counts & $\begin{array}{l}\text { Weighted } \\
\text { Mean } \\
\text { Diameter }\end{array}$ \\
\hline \begin{tabular}{|c|}
$0.30-0.49$ \\
\end{tabular} & 0.38 & 0.21 & 21599 & \multirow{6}{*}{1.76} \\
\hline $0.50-0.99$ & 0.70 & 0.30 & 17365 & \\
\hline $1.00-1.99$ & 1.41 & 0.30 & 3850 & \\
\hline $2.00-4.99$ & 3.16 & 0.40 & 7441 & \\
\hline $5.00-9.99$ & 7.07 & 0.30 & 1960 & \\
\hline$>10.00$ & 14.14 & 0.30 & 2318 & \\
\hline \multicolumn{5}{|l|}{ Sample \#7 } \\
\hline \begin{tabular}{|l} 
Bin. \\
Range \\
$(u m)$ \\
\end{tabular} & $\begin{array}{l}\text { Bin Mean } \\
\text { Diameter } \\
(u m)\end{array}$ & $\begin{array}{l}\text { Diff. of the } \\
\log \text { of the } \\
\text { Size }\end{array}$ & Counts & $\begin{array}{l}\text { Weighted } \\
\text { Mean } \\
\text { Diameter }\end{array}$ \\
\hline $0.30-0.49$ & 0.38 & 0.21 & 26874 & \multirow{6}{*}{1.18} \\
\hline $0.50-0.99$ & 0.70 & 0.30 & 18461 & \\
\hline $1.00-1.99$ & 1.41 & 0.30 & 2804 & \\
\hline $2.00-4.99$ & 3.16 & 0.40 & 4402 & \\
\hline $5.00-9.99$ & 7.07 & 0.30 & 1095 & \\
\hline$>10.00$ & 14.14 & 0.30 & 1102 & \\
\hline \multicolumn{5}{|l|}{ Sample \#g } \\
\hline \begin{tabular}{|l} 
Bin \\
Range \\
$($ (rm)
\end{tabular} & \begin{tabular}{|l|} 
Bin Mean \\
Diameter \\
$(\mu \mathrm{m})$
\end{tabular} & \begin{tabular}{|l} 
Diff. of the \\
$\log$ of the \\
Size
\end{tabular} & Counts & \begin{tabular}{|l|} 
Weighted \\
Mean \\
Diameter
\end{tabular} \\
\hline $0.30 \cdot 0.49$ & 0.38 & 0.21 & 31131 & \multirow{6}{*}{0.99} \\
\hline $0.50-0.99$ & 0.70 & 0.30 & 19808 & \\
\hline $1.00-1.99$ & 1.41 & 0.30 & 2587 & \\
\hline $2.00-4.99$ & 3.16 & 0.40 & 3585 & \\
\hline $5.00-9.99$ & 7.07 & 0.30 & 762 & \\
\hline$>10.00$ & 14.14 & 0.30 & 852 & \\
\hline
\end{tabular}

Sample \#2
\begin{tabular}{|c|c|c|r|r||}
\hline $\begin{array}{l}\text { Bin } \\
\text { Range } \\
(\mu \mathrm{m})\end{array}$ & $\begin{array}{l}\text { Bin Mean } \\
\text { Diameter } \\
(\mathrm{km})\end{array}$ & $\begin{array}{l}\text { Diff. of the } \\
\text { log of the } \\
\text { Size }\end{array}$ & Counts & $\begin{array}{l}\text { Weighted } \\
\text { Mean } \\
\text { Diameter }\end{array}$ \\
\hline $0.30-0.49$ & .38 & 0.21 & 21580 & \\
\hline $0.50-0.99$ & .70 & 0.30 & 18874 & \multirow{2}{*}{1.92} \\
\hline $1.00-1.99$ & 1.41 & 0.30 & 4981 & \\
\hline $2.00-4.99$ & 3.16 & 0.40 & 10284 & \\
\hline $5.00-9.99$ & 7.07 & 0.30 & 2679 & \\
\hline$>10.00$ & 14.14 & 0.30 & 2651 & \\
\hline
\end{tabular}

\begin{tabular}{|c|c|c|c|c|}
\hline \multicolumn{5}{|l|}{ Sample \#4 } \\
\hline $\begin{array}{l}\text { Bin } \\
\text { Range } \\
(\mu \mathrm{m})\end{array}$ & $\begin{array}{l}\text { Bin Mean } \\
\text { Diameter } \\
(\mu \mathrm{m})\end{array}$ & $\begin{array}{l}\text { Diff. of the } \\
\text { log of the } \\
\text { Size }\end{array}$ & Counts & $\begin{array}{l}\text { Weighted } \\
\text { Mean } \\
\text { Diameter }\end{array}$ \\
\hline $0.30-0.49$ & .38 & 0.21 & 18711 & \multirow{6}{*}{2.00} \\
\hline $0.50-0.99$ & .70 & 0.30 & 16739 & \\
\hline $1.00-1.99$ & 1.41 & 0.30 & 4785 & \\
\hline $2.00-4.99$ & 3.16 & 0.40 & 9886 & \\
\hline $5.00-9.99$ & 7.07 & 0.30 & 2728 & \\
\hline$>10.00$ & 14.14 & 0.30 & 2419 & \\
\hline
\end{tabular}

\begin{tabular}{|c|c|c|c|c|}
\hline \multicolumn{5}{|l|}{ Sample \#6 } \\
\hline $\begin{array}{l}\text { Bin } \\
\text { Range } \\
(\mu \mathrm{m})\end{array}$ & $\begin{array}{l}\text { Bin Mean } \\
\text { Diameter } \\
\text { (urm) }\end{array}$ & $\begin{array}{l}\text { Diff. of the } \\
\log \text { of the } \\
\text { Size }\end{array}$ & Counts & $\begin{array}{l}\text { Weighted } \\
\text { Mean } \\
\text { Diameter }\end{array}$ \\
\hline $0.30-0.49$ & .38 & 0.21 & 25039 & \multirow{6}{*}{1.34} \\
\hline $0.50-0.99$ & .70 & 0.30 & 17866 & \\
\hline $1.00-1.99$ & 1.41 & 0.30 & 3148 & \\
\hline $2.00-4.99$ & $\overline{3.16}$ & 0.40 & 5328 & \\
\hline $5.00-9.99$ & 7.07 & 0.30 & 1348 & \\
\hline$>10.00$ & 14.14 & 0.30 & 1409 & \\
\hline
\end{tabular}

\begin{tabular}{|c|c|c|c|c|}
\hline \multicolumn{5}{|l|}{ Sample \#8 } \\
\hline $\begin{array}{l}\text { Bin } \\
\text { Range } \\
(\mu \mathrm{m})\end{array}$ & $\begin{array}{l}\text { Bin Mean } \\
\text { Diameter } \\
\text { (um) }\end{array}$ & $\begin{array}{l}\text { Diff. of the } \\
\text { log of the } \\
\text { Size }\end{array}$ & Counts & $\begin{array}{l}\text { Woighted } \\
\text { Mean } \\
\text { Diameter }\end{array}$ \\
\hline $0.30-0.49$ & .38 & 0.21 & 27767 & \multirow{6}{*}{1.02} \\
\hline $0.50-0.99$ & .70 & 0.30 & 18417 & \\
\hline $0.00-1.99$ & 1.41 & 0.30 & 2482 & \\
\hline $2.00-4.99$ & 3.16 & 0.40 & 3551 & \\
\hline $5.00-9.99$ & 7.07 & 0.30 & 799 & \\
\hline$>10.00$ & 94.14 & 0.30 & 797 & \\
\hline
\end{tabular}

\begin{tabular}{|c|c|c|c|c|}
\hline \multicolumn{5}{|c|}{ Sample \#10 } \\
\hline $\begin{array}{l}\text { Bin } \\
\text { Range } \\
\text { (um) }\end{array}$ & $\begin{array}{l}\text { Bin Mean } \\
\text { Diameter } \\
(\text { (um) }\end{array}$ & $\begin{array}{l}\text { Diff. of the } \\
\log \text { of the } \\
\text { Size }\end{array}$ & Counts & $\begin{array}{l}\text { Weighted } \\
\text { Mean } \\
\text { Diameter }\end{array}$ \\
\hline $0.30-0.49$ & .38 & 0.21 & 29601 & \multirow{6}{*}{0.90} \\
\hline $0.50-0.99$ & .70 & 0.30 & 19329 & \\
\hline $1.00-1.99$ & 1.41 & 0.30 & 2365 & \\
\hline $2.00-4.99$ & 3.16 & 0.40 & 2916 & \\
\hline $5.00-9.99$ & 7.07 & 0.30 & 631 & \\
\hline$>10.00$ & 14.14 & 0.30 & 580 & \\
\hline
\end{tabular}


Data for Fig. 5. Resuspension and Ambient Air Vs. Height, PUREX,

Table 6. Peak Mean Diameter $(\mu \mathrm{m})$, Versus Sampling Height, and

Table 9. Percent of Large Particles in Breathing Zone, PUREX
$0.5 \mathrm{~m}$
with
$0.25 m$
$1.0 \mathrm{~m}$
with
$0.25 \mathrm{~m}$
$1.5 \mathrm{~m}$
with
$0.25 \mathrm{~m}$
$2.0 \mathrm{~m}$
with
$0.25 m$ 


\section{Aerosol Particle Measurement Record}

Description of sample location PUREX P\&O Gallery Reuspension $0.5 \mathrm{~m}$, Date_9/07/94, Time sample started 10:06:17, Temperature 82.8 F, Humidity $32.7 \%$, Dew Point $50.7 \mathrm{~F}$, Average Wind Velocity (if applicable) $.00 \mathrm{~m} / \mathrm{s}$, \# of physical barriers between sample area and outside (if applicable) $\underline{3}$

Sample \#1

\begin{tabular}{|c|c|c|c|c|}
\hline $\begin{array}{l}\text { Bin } \\
\text { Range } \\
\text { (um) }\end{array}$ & \begin{tabular}{|l|} 
Bin Mean \\
Diameter \\
$(u m)$
\end{tabular} & $\begin{array}{l}\text { Diff. of the } \\
\log \text { of the } \\
\text { Size }\end{array}$ & Counts & $\begin{array}{l}\text { Weighted } \\
\text { Mean } \\
\text { Diameter }\end{array}$ \\
\hline $0.30-0.49$ & 0.38 & 0.21 & 15753 & \multirow{6}{*}{0.67} \\
\hline $0.50-0.99$ & 0.70 & 0.30 & 9473 & \\
\hline $1.00-1.99$ & 1.41 & 0.30 & 1055 & \\
\hline $2.00 \cdot 4.99$ & 3.16 & 0.40 & 928 & \\
\hline $5.00-9.99$ & 7.07 & 0.30 & 88 & \\
\hline$>10.00$ & 14.14 & 0.30 & 49 & \\
\hline \multicolumn{5}{|l|}{ Sample \#3 } \\
\hline $\begin{array}{l}\text { Bin } \\
\text { Range } \\
(\mu \mathrm{m})\end{array}$ & \begin{tabular}{|l} 
Bin Mean \\
Diameter \\
$(\mu \mathrm{m})$
\end{tabular} & $\begin{array}{l}\text { Diff. of the } \\
\log \text { of the } \\
\text { Size }\end{array}$ & Counts & $\begin{array}{l}\text { Weighted } \\
\text { Mean } \\
\text { Diameter }\end{array}$ \\
\hline $0.30-0.49$ & 0.38 & 0.21 & 13678 & \multirow{6}{*}{1.33} \\
\hline $0.50-0.99$ & 0.70 & 0.30 & 15574 & \\
\hline $1.00-1.99$ & 1.41 & 0.30 & 4610 & \\
\hline $2.00-4.99$ & 3.16 & 0.40 & 7088 & \\
\hline $5.00-9.99$ & 7.07 & 0.30 & 889 & \\
\hline$>10.00$ & 14.14 & 0.30 & 343 & \\
\hline \multicolumn{5}{|l|}{ Sample $\# 5$} \\
\hline \begin{tabular}{|l} 
Bin \\
Range \\
$(\mu \mathrm{m})$
\end{tabular} & $\begin{array}{l}\text { Bin Mean } \\
\text { Diameter } \\
(\mu \mathrm{m})\end{array}$ & $\begin{array}{l}\text { Diff. of the } \\
\text { log of the } \\
\text { Size }\end{array}$ & Counts & $\begin{array}{l}\text { Weighted } \\
\text { Mean } \\
\text { Diameter }\end{array}$ \\
\hline $0.30-0.49$ & 0.38 & 0.21 & 15399 & \multirow{6}{*}{0.73} \\
\hline $0.50 \cdot 0.99$ & 0.70 & 0.30 & 9210 & \\
\hline $1.00-1.99$ & 1.41 & 0.30 & 1110 & \\
\hline $2.00-4.99$ & 3.16 & 0.40 & 1082 & \\
\hline $5.00-9.99$ & 7.07 & 0.30 & 138 & \\
\hline$>10.00$ & 14.14 & 0.30 & 103 & \\
\hline \multicolumn{5}{|l|}{ Sample \#7 } \\
\hline $\begin{array}{l}\text { Bin } \\
\text { Range } \\
\text { (um) } \\
\end{array}$ & $\begin{array}{l}\text { Bin Mean } \\
\text { Diameter } \\
(\mu \mathrm{m})\end{array}$ & \begin{tabular}{|l} 
Diff. of the \\
log of the \\
Size
\end{tabular} & Counts & $\begin{array}{l}\text { Weighted } \\
\text { Mean } \\
\text { Diameter }\end{array}$ \\
\hline $0.30-0.49$ & 0.38 & 0.21 & 16039 & \multirow{6}{*}{0.64} \\
\hline $0.50-0.99$ & 0.70 & 0.30 & 8950 & \\
\hline $1.00-1.99$ & 1.41 & 0.30 & 907 & \\
\hline $2.00-4.99$ & 3.16 & 0.40 & 819 & \\
\hline $5.00-9.99$ & 7.07 & 0.30 & 60 & \\
\hline$>10.00$ & 14.14 & 0.30 & 37 & \\
\hline
\end{tabular}

Sample \#2

\begin{tabular}{|c|c|c|c|c|}
\hline $\begin{array}{l}\text { Bin } \\
\text { Range } \\
(\mu \mathrm{m})\end{array}$ & $\begin{array}{l}\text { Bin Mean } \\
\text { Diameter } \\
(\text { (em) }\end{array}$ & $\begin{array}{l}\text { Diff. of the } \\
\log \text { of the } \\
\text { Size }\end{array}$ & Counts & $\begin{array}{l}\text { Weighted } \\
\text { Mean } \\
\text { Diameter }\end{array}$ \\
\hline $0.30-0.49$ & .38 & 0.21 & 13997 & \\
\hline $0.50-0.99$ & .70 & 0.30 & 15547 & \\
\hline $1.00-1.99$ & 1.41 & 0.30 & 5298 & \\
\hline $2.00-4.99$ & 3.16 & 0.40 & 8968 & \\
\hline $5.00-9.99$ & 7.07 & 0.30 & 1231 & \\
\hline$>10.00$ & 14.14 & 0.30 & 499 & \\
\hline
\end{tabular}

\section{Sample}

\begin{tabular}{|c|c|c|c|c|}
\hline $\begin{array}{l}\text { Bin } \\
\text { Range } \\
(\mu \mathrm{m})\end{array}$ & \begin{tabular}{|l|} 
Bin Mean \\
Diameter \\
$(\mathrm{um})$
\end{tabular} & $\begin{array}{l}\text { Diff. of the } \\
\text { log of the } \\
\text { Size }\end{array}$ & Counts & $\begin{array}{l}\text { Weighted } \\
\text { Mean } \\
\text { Diameter }\end{array}$ \\
\hline $0.30-0.49$ & .38 & 0.21 & 15884 & \multirow{6}{*}{0.63} \\
\hline $0.50-0.99$ & .70 & 0.30 & 9094 & \\
\hline $1.00-1.99$ & 1.41 & 0.30 & 951 & \\
\hline $2.00-4.99$ & 3.16 & 0.40 & 808 & \\
\hline $5.00-9.99$ & 7.07 & 0.30 & 55 & \\
\hline$>10.00$ & 14.14 & 0.30 & 21 & \\
\hline
\end{tabular}

$$
\text { Sample \#6 }
$$

\begin{tabular}{||c|c|c|r|r||}
\hline $\begin{array}{l}\text { Bin } \\
\text { Range } \\
(\mu \mathrm{m})\end{array}$ & $\begin{array}{l}\text { Bin Mean } \\
\text { Diameter } \\
(\mu \mathrm{m})\end{array}$ & $\begin{array}{l}\text { Diff. of the } \\
\text { log of the } \\
\text { Size }\end{array}$ & Counts & $\begin{array}{l}\text { Weighted } \\
\text { Mean } \\
\text { Diameter }\end{array}$ \\
\hline $0.30-0.49$ & .38 & 0.21 & 15704 & \\
\cline { 1 - 4 } $0.50-0.99$ & .70 & 0.30 & 9064 & \multirow{2}{*}{0.67} \\
\cline { 1 - 4 } $1.00-1.99$ & 1.41 & 0.30 & 1026 & \\
\hline $2.00-4.99$ & 3.16 & 0.40 & 959 & \\
\hline $5.00-9.99$ & 7.07 & 0.30 & 81 & \\
\hline$>10.00$ & 14.14 & 0.30 & 47 & \\
\hline
\end{tabular}

\begin{tabular}{|c|c|c|c|c|}
\hline \multicolumn{5}{|l|}{ Sample \#8 } \\
\hline $\begin{array}{l}\text { Bin } \\
\text { Range } \\
(\omega \mathrm{m})\end{array}$ & \begin{tabular}{|l|} 
Bin Mean \\
Diameter \\
$(\mu \mathrm{m})$
\end{tabular} & $\begin{array}{l}\text { Diff. of the } \\
\text { log of the } \\
\text { Size }\end{array}$ & Counts & $\begin{array}{l}\text { Weighted } \\
\text { Mean } \\
\text { Diameter }\end{array}$ \\
\hline $0.30-0.49$ & .38 & 0.21 & 15688 & \multirow{6}{*}{0.64} \\
\hline $0.50-0.99$ & .70 & 0.30 & 8862 & \\
\hline $0.00-1.99$ & 1.41 & 0.30 & 911 & \\
\hline $2.00-4.99$ & 3.16 & 0.40 & 840 & \\
\hline $5.00-9.99$ & 7.07 & 0.30 & 57 & \\
\hline$>10.00$ & 14.14 & 0.30 & 31 & \\
\hline
\end{tabular}




\section{Aerosol Particle Measurement Record}

Description of sample location PUREX P\&O Gallery Reuspension $0.25 \mathrm{~m}$,

Date 9/07/94, Time sample started 10:06:17, Temperature $82.8 \mathrm{~F}$, Humidity $32.7 \%$, Dew Point $50.7 \mathrm{~F}$, Average Wind Velocity (if applicable) $.00 \mathrm{~m} / \mathrm{s}$, \# of physical barriers between sample area and outside (if applicable) 3

Sample \#1

\begin{tabular}{|c|c|c|c|c|}
\hline $\begin{array}{l}\text { Bin } \\
\text { Range } \\
(\mu \mathrm{m})\end{array}$ & $\begin{array}{l}\text { Bin Mean } \\
\text { Diameter } \\
(\mu \mathrm{m})\end{array}$ & $\begin{array}{l}\text { Diff. of the } \\
\log \text { of the } \\
\text { Size }\end{array}$ & Counts & $\begin{array}{l}\text { Weighted } \\
\text { Mean } \\
\text { Diameter }\end{array}$ \\
\hline $0.30-0.49$ & 0.38 & 0.21 & 15124 & \multirow{6}{*}{0.64} \\
\hline $0.50-0.99$ & 0.70 & 0.30 & 3558 & \\
\hline $1.00-1.99$ & 1.41 & 0.30 & 941 & \\
\hline $2.00-4.99$ & 3.16 & 0.40 & 806 & \\
\hline $5.00 \cdot 9.99$ & 7.07 & 0.30 & 50 & \\
\hline$>10.00$ & 14.14 & 0.30 & 51 & \\
\hline \multicolumn{5}{|l|}{ Sample \#3 } \\
\hline $\begin{array}{l}\text { Bin } \\
\text { Range } \\
(\mu \mathrm{m})\end{array}$ & $\begin{array}{l}\text { Bin Mean } \\
\text { Diameter } \\
(\mu \mathrm{m})\end{array}$ & $\begin{array}{l}\text { Diff. of the } \\
\text { log of the } \\
\text { Size }\end{array}$ & Counts & $\begin{array}{l}\text { Weighted } \\
\text { Mean } \\
\text { Diameter }\end{array}$ \\
\hline $0.30-0.49$ & 0.38 & 0.21 & 15210 & \multirow{6}{*}{1.43} \\
\hline $0.50-0.99$ & 0.70 & 0.30 & 9871 & \\
\hline $1.00-1.99$ & 1.41 & 0.30 & 5502 & \\
\hline $2.00-4.99$ & 3.16 & 0.40 & 8012 & \\
\hline $5.00-9.99$ & 7.07 & 0.30 & 690 & \\
\hline$>10.00$ & 14.14 & 0.30 & 435 & \\
\hline \multicolumn{5}{|l|}{ Sample \#5 } \\
\hline $\begin{array}{l}\text { Bin } \\
\text { Range } \\
(\mu \mathrm{m})\end{array}$ & $\begin{array}{l}\text { Bin Mean } \\
\text { Diameter } \\
(\mu \mathrm{m})\end{array}$ & $\begin{array}{l}\text { Diff. of the } \\
\log \text { of the } \\
\text { Size }\end{array}$ & Counts & $\begin{array}{l}\text { Weighted } \\
\text { Mean } \\
\text { Diameter }\end{array}$ \\
\hline $0.30-0.49$ & 0.38 & 0.21 & 15117 & \multirow{6}{*}{0.79} \\
\hline $0.50-0.99$ & 0.70 & 0.30 & 4198 & \\
\hline $1.00-1.99$ & 1.41 & 0.30 & 1245 & \\
\hline $2.00-4.99$ & 3.16 & 0.40 & 1329 & \\
\hline $5.00-9.99$ & 7.07 & 0.30 & 82 & \\
\hline$>10.00$ & 14.14 & 0.30 & 153 & \\
\hline \multicolumn{5}{|l|}{ Sample \#7 } \\
\hline $\begin{array}{l}\text { Bin } \\
\text { Range } \\
\text { (um) }\end{array}$ & $\begin{array}{l}\text { Bin Mean } \\
\text { Diameter } \\
(\mu \mathrm{m})\end{array}$ & $\begin{array}{l}\text { Diff. of the } \\
\text { log of the } \\
\text { Size }\end{array}$ & Counts & $\begin{array}{l}\text { Weighted } \\
\text { Mean } \\
\text { Diameter }\end{array}$ \\
\hline $0.30-0.49$ & 0.38 & 0.21 & 14635 & \multirow{6}{*}{0.62} \\
\hline $0.50-0.99$ & 0.70 & 0.30 & 3538 & \\
\hline $1.00-1.99$ & 1.41 & 0.30 & 917 & \\
\hline $2.00-4.99$ & 3.16 & 0.40 & 707 & \\
\hline $5.00-9.99$ & 7.07 & 0.30 & 43 & \\
\hline$>10.00$ & 14.14 & 0.30 & 36 & \\
\hline
\end{tabular}

Sample \#2

\begin{tabular}{||c|c|c|r|r||}
\hline $\begin{array}{l}\text { Bin } \\
\text { Range } \\
(\mu \mathrm{m})\end{array}$ & $\begin{array}{l}\text { Bin Mean } \\
\text { Diameter } \\
(\mu \mathrm{m})\end{array}$ & $\begin{array}{l}\text { Diff. of the } \\
\text { log of the } \\
\text { Size }\end{array}$ & Counts & $\begin{array}{l}\text { Weighted } \\
\text { Mean } \\
\text { Diameter }\end{array}$ \\
\hline $0.30-0.49$ & .38 & 0.21 & 12564 & \\
\hline $0.50-0.99$ & .70 & 0.30 & 15300 \\
\hline $1.00-1.99$ & 1.41 & 0.30 & 13425 \\
\hline $2.00-4.99$ & 3.16 & 0.40 & 41556 \\
\hline $5.00-9.99$ & 7.07 & 0.30 & 12285 \\
\hline$>10.00$ & 14.14 & 0.30 & 26125 \\
\hline
\end{tabular}

Sample \#4

\begin{tabular}{|c|c|c|c|c|}
\hline $\begin{array}{l}\text { Bin } \\
\text { Range } \\
\text { (em) }\end{array}$ & $\begin{array}{l}\text { Bin Mean } \\
\text { Diameter } \\
(\mathrm{um})\end{array}$ & $\begin{array}{l}\text { Diff. of the } \\
\text { log of the } \\
\text { Size }\end{array}$ & Counts & $\begin{array}{l}\text { Weighted } \\
\text { Mean } \\
\text { Diameter }\end{array}$ \\
\hline $0.30-0.49$ & .38 & 0.21 & 15211 & \multirow{6}{*}{0.60} \\
\hline $0.50-0.99$ & .70 & 0.30 & 3384 & \\
\hline $1.00-1.99$ & 1.41 & 0.30 & 899 & \\
\hline $2.00-4.99$ & 3.16 & 0.40 & 694 & \\
\hline $5.00-9.99$ & 7.07 & 0.30 & 40 & \\
\hline$>10.00$ & $\overline{14.14}$ & 0.30 & 17 & \\
\hline
\end{tabular}

\begin{tabular}{|c|c|c|c|c|}
\hline $\begin{array}{l}\text { Bin } \\
\text { Range } \\
(\mu m)\end{array}$ & $\begin{array}{l}\text { Bin Mean } \\
\text { Diameter } \\
(\mu \mathrm{m})\end{array}$ & $\begin{array}{l}\text { Diff. of the } \\
\text { log of the } \\
\text { Size }\end{array}$ & Counts & $\begin{array}{l}\text { Weighted } \\
\text { Mean } \\
\text { Diameter }\end{array}$ \\
\hline $0.30-0.49$ & .38 & 0.21 & 14963 & \multirow{6}{*}{0.77} \\
\hline $0.50-0.99$ & .70 & 0.30 & 3996 & \\
\hline $1.00-1.99$ & 1.41 & 0.30 & 1177 & \\
\hline $2.00-4.99$ & 3.16 & 0.40 & $\overline{1240}$ & \\
\hline $5.00-9.99$ & 7.07 & 0.30 & 99 & \\
\hline$>10.00$ & 14.14 & 0.30 & 130 & \\
\hline
\end{tabular}

\begin{tabular}{|c|c|c|c|c|}
\hline \multicolumn{5}{|l|}{ Sample $\# 8$} \\
\hline \begin{tabular}{|l|} 
in \\
Range \\
$(\mu \mathrm{m})$
\end{tabular} & \begin{tabular}{|l|} 
Bin Mean \\
Diameter \\
$(\mathrm{f} m)$
\end{tabular} & $\begin{array}{l}\text { Diff. of the } \\
\text { log of the } \\
\text { Size }\end{array}$ & Counts & $\begin{array}{l}\text { Woighted } \\
\text { Mean } \\
\text { Diamoter }\end{array}$ \\
\hline $0.30-0.49$ & .38 & 0.21 & 14993 & \multirow{6}{*}{0.64} \\
\hline $0.50-0.99$ & .70 & 0.30 & 3609 & \\
\hline $0.00-1.99$ & 1.41 & 0.30 & 886 & \\
\hline $2.00-4.99$ & 3.16 & 0.40 & 787 & \\
\hline $5.00-9.99$ & 7.07 & 0.30 & 50 & \\
\hline$>10.00$ & 14.14 & 0.30 & 45 & \\
\hline
\end{tabular}




\section{Aerosol Particle Measurement Record}

Description of sample location PUREX P\&O Gallery Reuspension $1.0 \mathrm{~m}$, Date_9/07/94, Time sample started 10:21:23, Temperature_82.6 F, Humidity $36.3 \%$, Dew Point 55.4 F, Average Wind Velocity (if applicable) $.00 \mathrm{~m} / \mathrm{s}$, \# of physical barriers between sample area and outside (if applicable) $\underline{3}$

Sample \#1

\begin{tabular}{|c|c|c|c|c|}
\hline $\begin{array}{l}\text { Bin } \\
\text { Range } \\
(\mu \mathrm{m})\end{array}$ & \begin{tabular}{|l} 
Bin Mean \\
Diameter \\
$(\mu m)$
\end{tabular} & $\begin{array}{l}\text { Diff. of the } \\
\text { log of the } \\
\text { Size }\end{array}$ & Counts & $\begin{array}{l}\text { Weighted } \\
\text { Mean } \\
\text { Diameter }\end{array}$ \\
\hline $0.30-0.49$ & 0.38 & 0.21 & 19462 & \multirow{6}{*}{0.62} \\
\hline $0.50-0.99$ & 0.70 & 0.30 & 10946 & \\
\hline $1.00-1.99$ & 1.41 & 0.30 & 1046 & \\
\hline $2.00-4.99$ & 3.16 & 0.40 & 881 & \\
\hline $5.00-9.99$ & 7.07 & 0.30 & 60 & \\
\hline$>10.00$ & 14.14 & 0.30 & 29 & \\
\hline \multicolumn{5}{|l|}{ Sample \#3 } \\
\hline \begin{tabular}{|l} 
Bin \\
Range \\
( $\mu \mathrm{m})$
\end{tabular} & \begin{tabular}{|l|} 
Bin Mean \\
Diameter \\
$(\mu \mathrm{m})$
\end{tabular} & $\begin{array}{l}\text { Diff. of the } \\
\log \text { of the } \\
\text { Size }\end{array}$ & Counts & $\begin{array}{l}\text { Woighted } \\
\text { Mean } \\
\text { Diameter }\end{array}$ \\
\hline $0.30-0.49$ & 0.38 & 0.21 & 17026 & \\
\hline $0.50-0.99$ & 0.70 & 0.30 & 19224 & \\
\hline $1.00-1.99$ & 1.41 & 0.30 & 5704 & \\
\hline $2.00-4.99$ & 3.16 & 0.40 & 8045 & \\
\hline $5.00-9.99$ & 7.07 & 0.30 & 753 & \\
\hline$>10.00$ & 14.14 & 0.30 & 242 & \\
\hline \multicolumn{5}{|l|}{ Sample \#5 } \\
\hline $\begin{array}{l}\text { Bin } \\
\text { Range } \\
(\mu \mathrm{m})\end{array}$ & $\begin{array}{l}\text { Bin Mean } \\
\text { Diameter } \\
(\mu \mathrm{m})\end{array}$ & \begin{tabular}{|l|} 
Diff. of the \\
log of the \\
Size
\end{tabular} & Counts & \begin{tabular}{|l} 
Weighted \\
Mean \\
Diameter
\end{tabular} \\
\hline $0.30-0.49$ & 0.38 & 0.21 & 20840 & \multirow{6}{*}{0.59} \\
\hline $0.50-0.99$ & 0.70 & 0.30 & 11098 & \\
\hline $1.00-1.99$ & 1.41 & 0.30 & 906 & \\
\hline $2.00-4.99$ & 3.16 & 0.40 & 723 & \\
\hline $5.00-9.99$ & 7.07 & 0.30 & 58 & \\
\hline$>10.00$ & 14.14 & 0.30 & 19 & \\
\hline \multicolumn{5}{|l|}{ Sample \#7 } \\
\hline \begin{tabular}{|l} 
Bin \\
Range \\
$(\mu \mathrm{m})$ \\
\end{tabular} & $\begin{array}{l}\text { Bin Mean } \\
\text { Diameter } \\
(\mu \mathrm{m})\end{array}$ & \begin{tabular}{|l|} 
Diff. of the \\
log of the \\
Size
\end{tabular} & Counts & \begin{tabular}{|l|} 
Weighted \\
Mean \\
Diameter
\end{tabular} \\
\hline $0.30-0.49$ & $\begin{array}{l}0.38 \\
\end{array}$ & 0.21 & 22917 & \multirow{6}{*}{0.57} \\
\hline $0.50-0.99$ & 0.70 & 0.30 & 11936 & \\
\hline $1.00-1.99$ & 1.41 & 0.30 & 857 & \\
\hline $2.00-4.99$ & 3.16 & 0.40 & 687 & \\
\hline $5.00-9.99$ & 7.07 & 0.30 & 33 & \\
\hline$>10.00$ & 14.14 & 0.30 & 10 & \\
\hline
\end{tabular}

Sample \#2

Sample \#4

\begin{tabular}{|c|c|c|c|c|}
\hline \begin{tabular}{|l|} 
Bin \\
Range \\
$(\mu \mathrm{m})$
\end{tabular} & \begin{tabular}{|l} 
Bin Mean \\
Diameter \\
$(\mu \mathrm{m})$
\end{tabular} & $\begin{array}{l}\text { Diff. of the } \\
\log \text { of the } \\
\text { Size }\end{array}$ & Counts & $\begin{array}{l}\text { Weighted } \\
\text { Mean } \\
\text { Diameter }\end{array}$ \\
\hline $0.30-0.49$ & .38 & 0.21 & 20619 & \multirow{6}{*}{0.62 . } \\
\hline $0.50-0.99$ & .70 & 0.30 & 10906 & \\
\hline $1.00-1.99$ & 1.41 & 0.30 & 1034 & \\
\hline $2.00-4.99$ & 3.16 & 0.40 & $\overline{894}$ & \\
\hline $5.00-9.99$ & 7.07 & 0.30 & 63 & \\
\hline$>10.00$ & 14.14 & 0.30 & $\overline{43}$ & \\
\hline
\end{tabular}

\begin{tabular}{|c|c|c|c|c|}
\hline $\begin{array}{l}\text { Bin } \\
\text { Range } \\
(\mu \mathrm{m})\end{array}$ & \begin{tabular}{|l|} 
Bin Mean \\
Diameter \\
$(\mu m)$
\end{tabular} & $\begin{array}{l}\text { Diff. of the } \\
\text { log of the } \\
\text { Size }\end{array}$ & Counts & \begin{tabular}{|l} 
Weighted \\
Mean \\
Diameter
\end{tabular} \\
\hline $0.30-0.49$ & .38 & 0.21 & 22077 & \multirow{6}{*}{0.58} \\
\hline $0.50-0.99$ & .70 & 0.30 & 11807 & \\
\hline $1.00-1.99$ & 1.41 & 0.30 & 897 & \\
\hline $2.00-4.99$ & 3.16 & $0 . \overline{40}$ & 717 & \\
\hline $5.00-9.99$ & 7.07 & 0.30 & 40 & \\
\hline$>10.00$ & 14.14 & 0.30 & 18 & \\
\hline
\end{tabular}

\begin{tabular}{|c|c|c|c|c|}
\hline \begin{tabular}{|l} 
Bin \\
Range \\
$(\mu \mathrm{m})$
\end{tabular} & \begin{tabular}{|l} 
Bin Mean \\
Diameter \\
$(\mu \mathrm{m})$
\end{tabular} & $\begin{array}{l}\text { Diff. of the } \\
\text { log of the } \\
\text { Size }\end{array}$ & Counts & $\begin{array}{l}\text { Weighted } \\
\text { Mean } \\
\text { Diameter }\end{array}$ \\
\hline $0.30-0.49$ & .38 & 0.21 & 21936 & \multirow{6}{*}{0.66} \\
\hline $0.50-0.99$ & .70 & 0.30 & 12853 & \\
\hline $0.00-1.99$ & 1.41 & 0.30 & 1273 & \\
\hline $2.00-4.99$ & 3.16 & 0.40 & 1278 & \\
\hline $5.00-9.99$ & 7.07 & 0.30 & 105 & \\
\hline$>10.00$ & 14.14 & 0.30 & 71 & \\
\hline
\end{tabular}




\section{Aerosol Particle Measurement Record}

Description of sample location PUREX P\&O Gallery Reuspension $.25 \mathrm{~m}$,

Date_9/07/94, Time sample started 10:21:23, Temperature 82.6 F, Humidity 36.3\%, Dew Point $55.4 \mathrm{~F}$. Average Wind Velocity (if applicable) $.00 \mathrm{~m} / \mathrm{s}$, \# of physical barriers between sample area and outside (if applicable) $\underline{3}$

Sample \#1

\begin{tabular}{|c|c|c|c|c|}
\hline $\begin{array}{l}\text { Bin } \\
\text { Range } \\
\text { ( } \mu \mathrm{m})\end{array}$ & \begin{tabular}{|l|} 
Bin Mean \\
Diameter \\
$(\mu \mathrm{m})$
\end{tabular} & $\begin{array}{l}\text { Diff. of the } \\
\log \text { of the } \\
\text { Size }\end{array}$ & Counts & \begin{tabular}{|l|} 
Weighted \\
Mean \\
Diameter
\end{tabular} \\
\hline $0.30-0.49$ & 0.38 & 0.21 & 16738 & \multirow{6}{*}{0.62} \\
\hline $0.50 \cdot 0.99$ & 0.70 & 0.30 & 3947 & \\
\hline $1.00-1.99$ & 1.41 & 0.30 & 982 & \\
\hline $2.00-4.99$ & 3.16 & 0.40 & 793 & \\
\hline $5.00-9.99$ & 7.07 & 0.30 & 60 & \\
\hline$>10.00$ & 14.14 & 0.30 & 32 & \\
\hline
\end{tabular}

Sample \#3

\begin{tabular}{|c|c|c|c|c|}
\hline $\begin{array}{l}\text { Bin } \\
\text { Range } \\
(\mu \mathrm{m}) \\
\end{array}$ & \begin{tabular}{|l} 
Bin Mean \\
Diameter \\
$(\mu \mathrm{m})$
\end{tabular} & $\begin{array}{l}\text { Diff. of the } \\
\log \text { of the } \\
\text { Size }\end{array}$ & Counts & $\begin{array}{l}\text { Weighted } \\
\text { Mean } \\
\text { Diameter }\end{array}$ \\
\hline $0.30-0.49$ & 0.38 & 0.21 & 17653 & \multirow{6}{*}{1.30} \\
\hline $0.50-0.99$ & 0.70 & 0.30 & 10416 & \\
\hline $1.00-1.99$ & 1.41 & 0.30 & 5410 & \\
\hline $2.00-4.99$ & 3.16 & 0.40 & 7576 & \\
\hline $5.00-9.99$ & 7.07 & 0.30 & 634 & \\
\hline$>10.00$ & $\overline{14.14}$ & 0.30 & 339 & \\
\hline
\end{tabular}

\begin{tabular}{||l|l|l|r|l||}
\hline $\begin{array}{l}\text { Bin } \\
\text { Range } \\
(\mu \mathrm{m})\end{array}$ & $\begin{array}{l}\text { Bin Mean } \\
\text { Diameter } \\
(\mu \mathrm{m})\end{array}$ & $\begin{array}{l}\text { Diff. of the } \\
\text { log of the } \\
\text { Size }\end{array}$ & Counts & $\begin{array}{l}\text { Weighted } \\
\text { Mean } \\
\text { Diameter }\end{array}$ \\
\hline $0.30-0.49$ & 0.38 & 0.21 & 18952 & \\
\hline $0.50-0.99$ & 0.70 & 0.30 & 4243 & \multirow{2}{*}{0.59} \\
\hline $1.00-1.99$ & 1.41 & 0.30 & 975 & \\
\hline $2.00-4.99$ & 3.16 & 0.40 & 784 & \\
\hline $5.00-9.99$ & 7.07 & 0.30 & 31 & \\
\hline$>10.00$ & 14.14 & 0.30 & 37 & \\
\hline Sample \#7 & & & \\
\hline
\end{tabular}

\begin{tabular}{||l|l|l|r|r||}
\hline $\begin{array}{l}\text { Bin } \\
\text { Range } \\
(\mu \mathrm{m})\end{array}$ & $\begin{array}{l}\text { Bin Mean } \\
\text { Diameter } \\
(\mu \mathrm{m})\end{array}$ & $\begin{array}{l}\text { Diff. of the } \\
\text { log of the } \\
\text { Size }\end{array}$ & $\begin{array}{l}\text { Counts } \\
\text { Seighted } \\
\text { Mean } \\
\text { Diameter }\end{array}$ \\
\hline $0.30-0.49$ & 0.38 & 0.21 & 20000 & \\
\hline $0.50-0.99$ & 0.70 & 0.30 & 4341 & \multirow{2}{*}{0.58} \\
\hline $1.00-1.99$ & 1.41 & 0.30 & 956 & \\
\hline $2.00-4.99$ & 3.16 & 0.40 & 812 & \\
\hline $5.00-9.99$ & 7.07 & 0.30 & 38 & \\
\hline$>10.00$ & 14.14 & 0.30 & 32 & \\
\hline
\end{tabular}

Sample \#2

\begin{tabular}{|c|c|c|c|c|}
\hline $\begin{array}{l}\text { Bin } \\
\text { Range } \\
(\mu \mathrm{m})\end{array}$ & $\begin{array}{l}\text { Bin Mean } \\
\text { Diameter } \\
(\mu \mathrm{m})\end{array}$ & $\begin{array}{l}\text { Diff. of the } \\
\text { log of the } \\
\text { Size }\end{array}$ & Counts & $\begin{array}{l}\text { Weighted } \\
\text { Mean } \\
\text { Diameter }\end{array}$ \\
\hline $0.30-0.49$ & .38 & 0.21 & 14951 & \\
\hline $0.50-0.99$ & .70 & 0.30 & 18252 & \\
\hline $1.00-1.99$ & 1.41 & 0.30 & 15647 & \\
\hline $2.00-4.99$ & 3.16 & 0.40 & 46550 & \\
\hline $5.00-9.99$ & 7.07 & 0.30 & 13447 & \\
\hline$>10.00$ & 14.14 & 0.30 & 11811 & \\
\hline
\end{tabular}

Sample \#4

\begin{tabular}{|c|c|c|c|c|}
\hline $\begin{array}{l}\text { Bin } \\
\text { Range } \\
(\mu \mathrm{m})\end{array}$ & $\begin{array}{l}\begin{array}{l}\text { Bin Mean } \\
\text { Diameter } \\
(\mu \mathrm{m})\end{array} \\
\end{array}$ & $\begin{array}{l}\text { Diff. of the } \\
\log \text { of the } \\
\text { Size }\end{array}$ & Counts & $\begin{array}{l}\text { Weighted } \\
\text { Mean } \\
\text { Diameter }\end{array}$ \\
\hline $0.30-0.49$ & .38 & 0.21 & 18969 & \multirow{6}{*}{0.65} \\
\hline $0.50-0.99$ & .70 & 0.30 & 4659 & \\
\hline $1.00-1.99$ & 1.41 & 0.30 & 1233 & \\
\hline $2.00-4.99$ & 3.16 & 0.40 & 1079 & \\
\hline $5.00-9.99$ & 7.07 & 0.30 & 68 & \\
\hline$>10.00$ & 14.14 & 0.30 & 54 & \\
\hline
\end{tabular}

\begin{tabular}{||c|c|c|r|r||}
\hline $\begin{array}{l}\text { Bin } \\
\text { Range } \\
(\mu \mathrm{m})\end{array}$ & $\begin{array}{l}\text { Bin Mean } \\
\text { Diameter } \\
(\mu \mathrm{m})\end{array}$ & $\begin{array}{l}\text { Diff. of the } \\
\text { log of the } \\
\text { Size }\end{array}$ & Counts & $\begin{array}{l}\text { Weighted } \\
\text { Mean } \\
\text { Diameter }\end{array}$ \\
\hline $0.30-0.49$ & .38 & 0.21 & 19498 & \\
\hline $0.50-0.99$ & .70 & 0.30 & 4190 & \\
\hline $1.00-1.99$ & 1.41 & 0.30 & 891 & \multirow{2}{*}{0.57} \\
\hline $2.00-4.99$ & 3.16 & 0.40 & 701 & \\
\hline $5.00-9.99$ & 7.07 & 0.30 & 30 & \\
\hline$>10.00$ & 14.14 & 0.30 & 26 & \\
\hline
\end{tabular}

\begin{tabular}{|c|c|c|c|c|}
\hline \multicolumn{5}{|l|}{ Sample \#8 } \\
\hline $\begin{array}{l}\text { Bin } \\
\text { Range } \\
\text { ( } \mu \mathrm{m} \text { ) }\end{array}$ & \begin{tabular}{|l} 
Bin Mean \\
Diameter \\
$(\mu \mathrm{m})$
\end{tabular} & $\begin{array}{l}\text { Diff. of the } \\
\text { log of the } \\
\text { Size }\end{array}$ & Counts & \begin{tabular}{|l} 
Weighted \\
Moan \\
Diamoter
\end{tabular} \\
\hline $0.30-0.49$ & .38 & 0.21 & 20053 & \multirow{6}{*}{0.64} \\
\hline $0.50-0.99$ & .70 & 0.30 & 4720 & \\
\hline $0.00-1.99$ & 1.41 & 0.30 & 1201 & \\
\hline $2.00-4.99$ & 3.16 & 0.40 & 1034 & \\
\hline $5.00-9.99$ & 7.07 & 0.30 & 61 & \\
\hline$>10.00$ & 14.14 & 0.30 & 72 & \\
\hline
\end{tabular}




\section{Aerosol Particle Measurement Record}

Description of sample location PUREX P\&O Gallery Reuspension $1.5 \mathrm{~m}$,

Date 9/07/94, Time sample started 10:36:30, Temperature $82.2 \mathrm{~F}$, Humidity $37.8 \%$, Dew Point $53.8 \mathrm{~F}$. Average Wind Velocity (if applicable) $.00 \mathrm{~m} / \mathrm{s}$, \# of physical barriers between sample area and outside (if applicable) $\underline{3}$

Sample \#1

\begin{tabular}{|c|c|c|c|c|}
\hline $\begin{array}{l}\text { Bin } \\
\text { Range } \\
\text { (um) }\end{array}$ & $\begin{array}{l}\text { Bin Mean } \\
\text { Diameter } \\
\text { (erm) }\end{array}$ & $\begin{array}{l}\text { Diff. of the } \\
\text { log of the } \\
\text { Size }\end{array}$ & Counts & $\begin{array}{l}\text { Weighted } \\
\text { Mean } \\
\text { Diameter }\end{array}$ \\
\hline $0.30 \cdot 0.49$ & 0.38 & 0.21 & 25894 & \\
\hline $0.50-0.99$ & 0.70 & 0.30 & 13045 & \\
\hline $1.00-1.99$ & 1.41 & 0.30 & 833 & 0.56 \\
\hline $2.00 \cdot 4.99$ & 3.16 & 0.40 & 669 & \\
\hline $5.00-9.99$ & 7.07 & 0.30 & 35 & \\
\hline$>10.00$ & 14.14 & 0.30 & 22 & \\
\hline \multicolumn{5}{|l|}{ Sample $\# 3$} \\
\hline $\begin{array}{l}\text { Bin } \\
\text { Range } \\
(\omega \mathrm{m})\end{array}$ & $\begin{array}{l}\text { Bin Mean } \\
\text { Diameter } \\
(\mu \mathrm{m})\end{array}$ & $\begin{array}{l}\text { Diff. of the } \\
\text { log of the } \\
\text { Size }\end{array}$ & Counts & $\begin{array}{l}\text { Weighted } \\
\text { Mean } \\
\text { Diameter }\end{array}$ \\
\hline $0.30-0.49$ & 0.38 & 0.21 & 26976 & \multirow{6}{*}{0.55} \\
\hline $0.50-0.99$ & 0.70 & 0.30 & 13219 & \\
\hline $1.00-1.99$ & 1.41 & 0.30 & 758 & \\
\hline $2.00-4.99$ & 3.16 & 0.40 & 523 & \\
\hline $5.00-9.99$ & 7.07 & 0.30 & 31 & \\
\hline$>10.00$ & 14.14 & 0.30 & 13 & \\
\hline \multicolumn{5}{|l|}{ Sample \#5 } \\
\hline $\begin{array}{l}\text { Bin } \\
\text { Range } \\
(\mu \mathrm{m})\end{array}$ & $\begin{array}{l}\text { Bin Mean } \\
\text { Diameter } \\
(u m)\end{array}$ & $\begin{array}{l}\text { Diff. of the } \\
\log \text { of the } \\
\text { Size }\end{array}$ & Counts & $\begin{array}{l}\text { Weighted } \\
\text { Mean } \\
\text { Diameter }\end{array}$ \\
\hline $0.30-0.49$ & 0.38 & 0.21 & 26467 & \\
\hline $0.50-0.99$ & 0.70 & 0.30 & 13452 & \\
\hline $1.00-1.99$ & 1.41 & 0.30 & 873 & \\
\hline $2.00-4.99$ & 3.16 & 0.40 & 658 & \\
\hline $5.00-9.99$ & 7.07 & 0.30 & 51 & \\
\hline$>10.00$ & 14.14 & 0.30 & 31 & \\
\hline \multicolumn{5}{|l|}{ Sample \#7 } \\
\hline \begin{tabular}{|l|} 
Bin \\
Range \\
$(\mu \mathrm{m})$
\end{tabular} & \begin{tabular}{|l|} 
Bin Mean \\
Diameter \\
(um)
\end{tabular} & $\begin{array}{l}\text { Diff. of the } \\
\text { log of the } \\
\text { Size }\end{array}$ & Counts & $\begin{array}{l}\text { Weighted } \\
\text { Mean } \\
\text { Diameter }\end{array}$ \\
\hline $0.30-0.49$ & 0.38 & 0.21 & 28020 & \multirow{6}{*}{0.53} \\
\hline $0.50-0.99$ & 0.70 & 0.30 & 13385 & \\
\hline $1.00-1.99$ & 1.41 & 0.30 & 604 & \\
\hline $2.00-4.99$ & 3.16 & 0.40 & 425 & \\
\hline $5.00-9.99$ & 7.07 & 0.30 & 20 & \\
\hline$>10.00$ & 14.14 & 0.30 & 4 & \\
\hline \multicolumn{5}{|l|}{ Sample \#9 } \\
\hline \begin{tabular}{|l} 
Bin \\
Range \\
$(\mu \mathrm{m})$
\end{tabular} & $\begin{array}{l}\text { Bin Mean } \\
\text { Diameter } \\
(\text { (um) }\end{array}$ & $\begin{array}{l}\text { Diff. of the } \\
\log \text { of the } \\
\text { Size }\end{array}$ & Counts & $\begin{array}{l}\text { Weighted } \\
\text { Mean } \\
\text { Diameter }\end{array}$ \\
\hline $0.30 \cdot 0.49$ & 0.38 & 0.21 & 27377 & \multirow{6}{*}{0.56} \\
\hline $0.50-0.99$ & 0.70 & 0.30 & 13599 & \\
\hline $1.00 \cdot 1.99$ & 1.41 & 0.30 & 847 & \\
\hline $2.00-4.99$ & 3.16 & 0.40 & 689 & \\
\hline $5.00-9.99$ & 7.07 & 0.30 & 46 & \\
\hline$>10.00$ & 14.14 & 0.30 & 25 & \\
\hline
\end{tabular}

Sample \#2

\begin{tabular}{|c|c|c|c|c|}
\hline $\begin{array}{l}\text { Bin } \\
\text { Range } \\
(\mu \mathrm{m})\end{array}$ & $\begin{array}{l}\text { Bin Mean } \\
\text { Diameter } \\
(u \mathrm{~m})\end{array}$ & $\begin{array}{l}\text { Diff. of the } \\
\text { log of the } \\
\text { Size }\end{array}$ & Counts & $\begin{array}{l}\text { Weighted } \\
\text { Mean } \\
\text { Diameter }\end{array}$ \\
\hline $0.30-0.49$ & .38 & 0.21 & 27627 & \multirow{6}{*}{0.53} \\
\hline $0.50-0.99$ & .70 & 0.30 & 13297 & \\
\hline $1.00-1.99$ & 1.41 & 0.30 & 704 & \\
\hline $2.00-4.99$ & 3.16 & 0.40 & 433 & \\
\hline $5.00-9.99$ & 7.07 & 0.30 & 23 & \\
\hline$>10.00$ & 14.14 & 0.30 & 6 & \\
\hline
\end{tabular}

\begin{tabular}{|c|c|c|c|c|}
\hline \multicolumn{5}{|l|}{ Sample $\# 4$} \\
\hline $\begin{array}{l}\text { Bin } \\
\text { Range } \\
(u \mathrm{~m})\end{array}$ & $\begin{array}{l}\text { Bin Mean } \\
\text { Diameter } \\
(u m)\end{array}$ & $\begin{array}{l}\text { Diff. of the } \\
\text { log of the } \\
\text { Size }\end{array}$ & Counts & $\begin{array}{l}\text { Weighted } \\
\text { Mean } \\
\text { Diameter }\end{array}$ \\
\hline $0.30-0.49$ & .38 & 0.21 & 27552 & \multirow{6}{*}{0.54} \\
\hline $0.50-0.99$ & .70 & 0.30 & 13297 & \\
\hline $1.00-1.99$ & 1.41 & 0.30 & 694 & \\
\hline $2.00-4.99$ & 3.16 & 0.40 & 487 & \\
\hline $5.00-9.99$ & 7.07 & 0.30 & 29 & \\
\hline$>10.00$ & $\overline{14.14}$ & 0.30 & 6 & \\
\hline
\end{tabular}

\begin{tabular}{|c|c|c|c|c|}
\hline \multicolumn{5}{|l|}{ Sample \#6 } \\
\hline $\begin{array}{l}\text { Bin } \\
\text { Range } \\
\text { (um) }\end{array}$ & \begin{tabular}{|l|} 
Bin Mean \\
Diameter \\
$(\mu \mathrm{m})$
\end{tabular} & $\begin{array}{l}\text { Diff. of the } \\
\text { log of the } \\
\text { Size }\end{array}$ & Counts & $\begin{array}{l}\text { Weighted } \\
\text { Mean } \\
\text { Diameter }\end{array}$ \\
\hline $0.30-0.49$ & .38 & 0.21 & 26981 & \multirow{6}{*}{0.54} \\
\hline $0.50-0.99$ & .70 & 0.30 & 13212 & \\
\hline $1.00-1.99$ & 1.41 & 0.30 & 718 & \\
\hline $2.00-4.99$ & 3.16 & 0.40 & 475 & \\
\hline $5.00-9.99$ & 7.07 & 0.30 & 26 & \\
\hline$>10.00$ & 14.14 & 0.30 & 5 & \\
\hline
\end{tabular}

Sample \#8

\begin{tabular}{|c|c|c|c|c|}
\hline $\begin{array}{l}\text { Bin } \\
\text { Range } \\
\text { (vm) }\end{array}$ & \begin{tabular}{|l} 
Bin Mean \\
Diameter \\
$(u \mathrm{~m})$
\end{tabular} & $\begin{array}{l}\text { Diff. of the } \\
\log \text { of the } \\
\text { Size }\end{array}$ & Counts & \begin{tabular}{|l} 
Weighted \\
Mean \\
Diameter
\end{tabular} \\
\hline $0.30 \cdot 0.49$ & .38 & 0.21 & 27794 & \multirow{6}{*}{0.53} \\
\hline $0.50-0.99$ & .70 & 0.30 & 13390 & \\
\hline $0.00-1.99$ & 1.41 & 0.30 & 693 & \\
\hline $2.00-4.99$ & 3.16 & 0.40 & 448 & \\
\hline $5.00-9.99$ & 7.07 & 0.30 & 20 & \\
\hline$>10.00$ & 14.14 & 0.30 & 8 & \\
\hline
\end{tabular}

\begin{tabular}{|c|c|c|c|c|}
\hline \multicolumn{5}{|c|}{ Sample $\# 10$} \\
\hline $\begin{array}{l}\text { Bin } \\
\text { Range } \\
\text { (uml) }\end{array}$ & $\begin{array}{l}\text { Bin Mean } \\
\text { Diameter } \\
(\mathrm{um})\end{array}$ & $\begin{array}{l}\text { Diff. of the } \\
\text { log of the } \\
\text { Size }\end{array}$ & Counts & $\begin{array}{l}\text { Weighted } \\
\text { Mean . } \\
\text { Diameter }\end{array}$ \\
\hline $0.30-0.49$ & .38 & 0.21 & 27621 & \multirow{6}{*}{0.54} \\
\hline $0.50-0.99$ & .70 & 0.30 & 13527 & \\
\hline $1.00-1.99$ & 1.41 & 0.30 & 652 & \\
\hline $2.00-4.99$ & 3.16 & 0.40 & 516 & \\
\hline $5.00-9.99$ & 7.07 & 0.30 & 25 & \\
\hline$>10.00$ & 14.14 & 0.30 & $\overline{12}$ & \\
\hline
\end{tabular}




\section{Aerosol Particle Measurement Record}

Description of sample location PUREX P\&O Gallery Reuspension $.25 \mathrm{~m}$, Date 9/07/94, Time sample started 10:36:30, Temperature 82.2 F, Humidity $37.8 \%$, Dew Point 53.8 F. Average Wind.Velocity (if applicable) $.00 \mathrm{~m} / \mathrm{s}$, \# of physical barriers between sample area and outside (if applicable) $\underline{3}$

Sample \#1

\begin{tabular}{|c|c|c|c|c|}
\hline $\begin{array}{l}\text { Bin } \\
\text { Range } \\
\text { (um) }\end{array}$ & \begin{tabular}{|l} 
Bin Mean \\
Diameter \\
$(\mu \mathrm{m})$
\end{tabular} & $\begin{array}{l}\text { Diff. of the } \\
\log \text { of the } \\
\text { Size }\end{array}$ & Counts & $\begin{array}{l}\text { Weighted } \\
\text { Mean } \\
\text { Diameter }\end{array}$ \\
\hline $0.30 \cdot 0.49$ & 0.38 & 0.21 & 22059 & \multirow{6}{*}{0.56} \\
\hline $0.50-0.99$ & 0.70 & 0.30 & 4594 & \\
\hline $1.00 \cdot 1.99$ & 1.41 & 0.30 & 917 & \\
\hline $2.00-4.99$ & 3.16 & 0.40 & 675 & \\
\hline $5.00-9.99$ & 7.07 & 0.30 & 40 & \\
\hline$>10.00$ & 14.14 & 0.30 & 32 & \\
\hline \multicolumn{5}{|l|}{ Sample \#3 } \\
\hline \begin{tabular}{|l|} 
Bin \\
Range \\
$(\mu \mathrm{m})$
\end{tabular} & \begin{tabular}{|l|} 
Bin Mean \\
Diameter \\
$(\mathrm{um})$
\end{tabular} & $\begin{array}{l}\text { Diff. of the } \\
\log \text { of the } \\
\text { Size }\end{array}$ & Counts & $\begin{array}{l}\text { Weighted } \\
\text { Mean } \\
\text { Diameter }\end{array}$ \\
\hline $0.30-0.49$ & \begin{tabular}{|l|}
0.38 \\
\end{tabular} & 0.21 & 22555 & \multirow{6}{*}{0.67} \\
\hline $0.50-0.99$ & 0.70 & 0.30 & 5425 & \\
\hline $1.00-1.99$ & 1.41 & 0.30 & 1360 & \\
\hline $2.00 \cdot 4.99$ & 3.16 & 0.40 & 1380 & \\
\hline $5.00-9.99$ & 7.07 & 0.30 & 85 & \\
\hline$>.10 .00$ & 14.14 & 0.30 & 96 & \\
\hline \multicolumn{5}{|l|}{ Sample \#5 } \\
\hline $\begin{array}{l}\begin{array}{l}\text { Bin } \\
\text { Range } \\
(\mu m)\end{array} \\
\end{array}$ & \begin{tabular}{|l|} 
Bin Mean \\
Diameter \\
$(u m)$
\end{tabular} & \begin{tabular}{|l|} 
Diff. of the \\
log of the \\
Size
\end{tabular} & Counts & \begin{tabular}{|l|} 
Weighted \\
Mean \\
Diameter
\end{tabular} \\
\hline $0.30 \cdot 0.49$ & \begin{tabular}{|l|}
0.38 \\
\end{tabular} & 0.21 & 22603 & \multirow{6}{*}{0.61} \\
\hline $0.50-0.99$ & 0.70 & 0.30 & 5047 & \\
\hline $1.00-1.99$ & 1.41 & 0.30 & 1174 & \\
\hline $2.00 \cdot 4.99$ & 3.16 & 0.40 & 968 & \\
\hline $5.00-9.99$ & 7.07 & 0.30 & 63 & \\
\hline$>10.00$ & 14.14 & 0.30 & 72 & \\
\hline
\end{tabular}

\begin{tabular}{|c|c|c|c|c|}
\hline \begin{tabular}{|l|} 
Bin \\
Range \\
(um)
\end{tabular} & \begin{tabular}{|l|} 
Bin Mean \\
Diameter \\
$(\mu \mathrm{m})$
\end{tabular} & $\begin{array}{l}\text { Diff. of the } \\
\text { log of the } \\
\text { Size }\end{array}$ & Counts & $\begin{array}{l}\text { Weighted } \\
\text { Mean } \\
\text { Diameter }\end{array}$ \\
\hline $0.30-0.49$ & 0.38 & 0.21 & 22949 & \multirow{6}{*}{0.55} \\
\hline $0.50-0.99$ & 0.70 & 0.30 & 4612 & \\
\hline $1.00-1.99$ & 1.41 & 0.30 & 907 & \\
\hline $2.00-4.99$ & 3.16 & 0.40 & 664 & \\
\hline $5.00-9.99$ & 7.07 & 0.30 & 50 & \\
\hline$>10.00$ & 14.14 & 0.30 & 33 & \\
\hline \multicolumn{5}{|l|}{ Sample \#9 } \\
\hline $\begin{array}{l}\text { Bin } \\
\text { Range } \\
\text { (um) }\end{array}$ & $\begin{array}{l}\text { Bin Mean } \\
\text { Diamoter } \\
(\mathrm{mm})\end{array}$ & \begin{tabular}{|l|} 
Diff. of the \\
log of the \\
Size
\end{tabular} & Counts & $\begin{array}{l}\text { Weighted } \\
\text { Mean } \\
\text { Diameter }\end{array}$ \\
\hline $0.30-0.49$ & 0.38 & 0.21 & 23043 & \multirow{6}{*}{0.80} \\
\hline $0.50-0.99$ & 0.70 & 0.30 & 6279 & \\
\hline $1.00-1.99$ & 1.41 & 0.30 & 2003 & \\
\hline $2.00-4.99$ & 3.16 & 0.40 & 2294 & \\
\hline $5.00-9.99$ & 7.07 & 0.30 & 190 & \\
\hline$>10.00$ & 14.14 & 0.30 & 183 & \\
\hline
\end{tabular}

Sample \#2

\begin{tabular}{|c|c|c|c|c|}
\hline $\begin{array}{l}\text { Bin } \\
\text { Range } \\
\text { (um) }\end{array}$ & \begin{tabular}{|l|} 
Bin Mean \\
Diameter \\
$(u m)$
\end{tabular} & $\begin{array}{l}\text { Diff. of the } \\
\log \text { of the } \\
\text { Size }\end{array}$ & Counts & $\begin{array}{l}\text { Weighted } \\
\text { Moan } \\
\text { Diameter }\end{array}$ \\
\hline $0.30-0.49$ & .38 & 0.21 & 16628 & \\
\hline $0.50-0.99$ & .70 & 0.30 & 30029 & \\
\hline $1.00-1.99$ & 1.41 & 0.30 & 28174 & \\
\hline $2.00-4.99$ & 3.16 & 0.40 & 73469 & \\
\hline $5.00-9.99$ & 7.07 & 0.30 & 19911 & \\
\hline$>10.00$ & 14.14 & 0.30 & 19848 & \\
\hline
\end{tabular}

\begin{tabular}{|c|c|c|c|c|}
\hline \\
\hline $\begin{array}{l}\text { Bin } \\
\text { Range } \\
\text { (urm) }\end{array}$ & $\begin{array}{l}\text { Bin Mean } \\
\text { Diameter } \\
(\mathrm{ym})\end{array}$ & $\begin{array}{l}\text { Diff. of the } \\
\log \text { of the } \\
\text { Size }\end{array}$ & Counts & \begin{tabular}{|l} 
Weighted \\
Mean \\
Diameter
\end{tabular} \\
\hline $0.30-0.49$ & .38 & 0.21 & 22547 & \multirow{6}{*}{0.61} \\
\hline $0.50-0.99$ & .70 & 0.30 & 4840 & \\
\hline $1.00-1.99$ & 1.41 & 0.30 & 1140 & \\
\hline $2.00-4.99$ & 3.16 & 0.40 & $\overline{942}$ & \\
\hline $5.00-9.99$ & 7.07 & 0.30 & 55 & \\
\hline$>10.00$ & 14.14 & 0.30 & 88 & \\
\hline
\end{tabular}

\begin{tabular}{|c|c|c|c|c|}
\hline \multicolumn{5}{|l|}{ Sample \#6 } \\
\hline $\begin{array}{l}\text { Bin } \\
\text { Range } \\
\text { (um) }\end{array}$ & \begin{tabular}{|l} 
Bin Mean \\
Diameter \\
$($ (rm)
\end{tabular} & $\begin{array}{l}\text { Diff. of the } \\
\text { log of the } \\
\text { Size }\end{array}$ & Counts & $\begin{array}{l}\text { Weighted } \\
\text { Mean } \\
\text { Diameter }\end{array}$ \\
\hline $0.30-0.49$ & .38 & 0.21 & 23050 & \multirow{6}{*}{0.54} \\
\hline $0.50-0.99$ & .70 & 0.30 & 4669 & \\
\hline $1.00-1.99$ & 9.41 & 0.30 & 809 & \\
\hline $2.00-4.99$ & 3.16 & $\overline{0.40}$ & 608 & \\
\hline $5.00-9.99$ & 7.07 & 0.30 & 38 & \\
\hline$>10.00$ & $\overline{14.14}$ & 0.30 & 32 & \\
\hline
\end{tabular}

\begin{tabular}{|c|c|c|c|c|}
\hline \multicolumn{5}{|c|}{ Sample \#8 } \\
\hline $\begin{array}{l}\text { Bin } \\
\text { Range } \\
\text { (um) }\end{array}$ & \begin{tabular}{|l|} 
Bin Mean \\
Diameter \\
$(\mathrm{um})$
\end{tabular} & $\begin{array}{l}\text { Diff. of the } \\
\log \text { of the } \\
\text { Size }\end{array}$ & Counts & $\begin{array}{l}\text { Weighted } \\
\text { Mean } \\
\text { Diameter }\end{array}$ \\
\hline $0.30-0.49$ & .38 & 0.21 & 23274 & \multirow{6}{*}{0.66} \\
\hline $0.50-0.99$ & .70 & 0.30 & 5220 & \\
\hline $0.00-1.99$ & 1.41 & 0.30 & 1288 & \\
\hline $2.00-4.99$ & 3.16 & 0.40 & 1284 & \\
\hline $5.00-9.99$ & 7.07 & 0.30 & 116 & \\
\hline$>10.00$ & 14.14 & 0.30 & 104 & \\
\hline
\end{tabular}

\section{Sample \#10}

\begin{tabular}{|c|c|c|c|c|}
\hline $\begin{array}{l}\text { Bin } \\
\text { Range } \\
(\mu \mathrm{m})\end{array}$ & \begin{tabular}{|l|} 
Bin Mean \\
Diameter \\
$(u m)$
\end{tabular} & $\begin{array}{l}\text { Diff. of the } \\
\log \text { of the } \\
\text { Size }\end{array}$ & Counts & $\begin{array}{l}\text { Weighted } \\
\text { Mean } \\
\text { Diameter }\end{array}$ \\
\hline $0.30-0.49$ & .38 & 0.21 & 23632 & \multirow{6}{*}{0.51} \\
\hline $0.50-0.99$ & .70 & 0.30 & 4509 & \\
\hline $1.00-1.99$ & 1.41 & 0.30 & 763 & \\
\hline $2.00-4.99$ & 3.16 & 0.40 & 481 & \\
\hline $5.00-9.99$ & 7.07 & 0.30 & 24 & \\
\hline$>10.00$ & 14.14 & 0.30 & 14 & \\
\hline
\end{tabular}




\section{Aerosol Particle Measurement Record}

Description of sample location_PUREX P\&O Gallery Reuspension $2.0 \mathrm{~m}$, Date 9/07/94, Time sample started 10:51:01, Temperature 82.1 F, Humidity $38.8 \%$, Dew Point $54.5 \mathrm{~F}$, Average Wind Velocity (if applicable) $.00 \mathrm{~m} / \mathrm{s}$, \# of physical barriers between sample area and outside (if applicable) $\underline{3}$

Sample \#

\begin{tabular}{|c|c|c|c|c|}
\hline $\begin{array}{l}\text { Bin } \\
\text { Range } \\
(\mu \mathrm{m})\end{array}$ & \begin{tabular}{|l|} 
Bin Mean \\
Diameter \\
urml
\end{tabular} & $\begin{array}{l}\text { Diff. of the } \\
\text { log of the } \\
\text { Size }\end{array}$ & Counts & $\begin{array}{l}\text { Woighted } \\
\text { Mean } \\
\text { Diameter }\end{array}$ \\
\hline $0.30-0.49$ & 0.38 & 0.21 & 29961 & \multirow{6}{*}{0.52} \\
\hline $0.50-0.99$ & 0.70 & 0.30 & 13860 & \\
\hline $1.00-1.99$ & 1.41 & 0.30 & 591 & \\
\hline $2.00-4.99$ & 3.16 & 0.40 & 377 & \\
\hline $5.00-9.99$ & 7.07 & 0.30 & 26 & \\
\hline$>10.00$ & 14.14 & 0.30 & 11 & \\
\hline \multicolumn{5}{|l|}{ Sample \#3 } \\
\hline $\begin{array}{l}\text { Bin } \\
\text { Range } \\
(\mu \mathrm{m})\end{array}$ & \begin{tabular}{|l|} 
Bin Mean \\
Diamoter \\
(um)
\end{tabular} & $\begin{array}{l}\text { Diff. of the } \\
\text { log of the } \\
\text { Size }\end{array}$ & Counts & $\begin{array}{l}\text { Weighted } \\
\text { Mean } \\
\text { Diameter }\end{array}$ \\
\hline 0.30 .0 .49 & 0.38 & 0.21 & 29737 & \multirow{6}{*}{0.55} \\
\hline $0.50-0.99$ & 0.70 & 0.30 & 14417 & \\
\hline $1.00-1.99$ & 1.41 & 0.30 & 776 & \\
\hline $2.00-4.99$ & 3.16 & 0.40 & 608 & \\
\hline $5.00-9.99$ & 7.07 & 0.30 & 55 & \\
\hline$>10.00$ & 14.14 & 0.30 & 16 & \\
\hline \multicolumn{5}{|l|}{ Sample \#5 } \\
\hline $\begin{array}{l}\text { Bin } \\
\text { Range } \\
(\mathrm{mm})\end{array}$ & $\begin{array}{l}\text { Bin Moan } \\
\text { Diameter } \\
(\mu \mathrm{m})\end{array}$ & $\begin{array}{l}\text { Diff. of the } \\
\log \text { of the } \\
\text { Size }\end{array}$ & Counts & $\begin{array}{l}\text { Weighted } \\
\text { Mean } \\
\text { Diameter }\end{array}$ \\
\hline $0.30 \cdot 0.49$ & 0.38 & 0.21 & 30062 & \multirow{6}{*}{0.55} \\
\hline $0.50-0.99$ & 0.70 & 0.30 & 14426 & \\
\hline $1.00-1.99$ & 1.41 & 0.30 & 661 & \\
\hline $2.00-4.99$ & 3.16 & 0.40 & 535 & \\
\hline $5.00-9.99$ & 7.07 & 0.30 & 61 & \\
\hline$>10.00$ & 14.14 & 0.30 & 48 & \\
\hline \multicolumn{5}{|l|}{ Sample \#7 } \\
\hline \begin{tabular}{|l} 
Bin \\
Range \\
$(\mu \mathrm{m})$
\end{tabular} & \begin{tabular}{|l|} 
Bin Mean \\
Diameter \\
(um)
\end{tabular} & $\begin{array}{l}\text { Diif. of the } \\
\text { log of the } \\
\text { Size }\end{array}$ & Counts & \begin{tabular}{|l|} 
Weighted \\
Mean \\
Diameter
\end{tabular} \\
\hline $0.30-0.49$ & 0.38 & 0.21 & 34302 & \multirow{6}{*}{0.52} \\
\hline $0.50-0.99$ & 0.70 & 0.30 & 15905 & \\
\hline $1.00-1.99$ & 1.41 & 0.30 & 581 & \\
\hline $2.00-4.99$ & 3.16 & 0.40 & 383 & \\
\hline $5.00-9.99$ & 7.07 & 0.30 & 17 & \\
\hline$>10.00$ & 14.14 & 0.30 & 15 & \\
\hline \multicolumn{5}{|l|}{ Sample \#9 } \\
\hline $\begin{array}{l}\text { Bin } \\
\text { Range } \\
(\mu \mathrm{m})\end{array}$ & \begin{tabular}{|l|} 
Bin Mean \\
Diameter \\
$($ Um)
\end{tabular} & $\begin{array}{l}\text { Diff. of the } \\
\log \text { of the } \\
\text { Size }\end{array}$ & Counts & \begin{tabular}{|l|} 
Weighted \\
Mean \\
Diameter
\end{tabular} \\
\hline $0.30-0.49$ & 0.38 & 0.21 & 31654 & \multirow{6}{*}{0.52} \\
\hline $0.50-0.99$ & 0.70 & 0.30 & 15144 & \\
\hline $1.00-1.99$ & 1.41 & 0.30 & 564 & \\
\hline $2.00-4.99$ & 3.16 & 0.40 & 381 & \\
\hline $5.00-9.99$ & 7.07 & 0.30 & 18 & \\
\hline$>10.00$ & 14.14 & 0.30 & 18 & \\
\hline
\end{tabular}

Sample \#2

\begin{tabular}{|c|c|c|c|c|}
\hline $\begin{array}{l}\text { Bin } \\
\text { Range } \\
\text { (um) }\end{array}$ & \begin{tabular}{|l|} 
Bin Mean \\
Diameter \\
$(u m)$
\end{tabular} & $\begin{array}{l}\text { Diff. of the } \\
\log \text { of the } \\
\text { Size }\end{array}$ & Counts & $\begin{array}{l}\text { Weighted } \\
\text { Mean } \\
\text { Diameter }\end{array}$ \\
\hline $0.30-0.49$ & .38 & 0.21 & 29735 & \multirow{6}{*}{0.52} \\
\hline $0.50-0.99$ & .70 & 0.30 & 13995 & \\
\hline $1.00-1.99$ & 1.41 & 0.30 & 560 & \\
\hline $2.00-4.99$ & 3.16 & 0.40 & 392 & \\
\hline $5.00-9.99$ & 7.07 & 0.30 & 20 & \\
\hline$>10.00$ & 14.14 & 0.30 & 3 & \\
\hline
\end{tabular}

Sample \#4

\begin{tabular}{|c|c|c|c|c|}
\hline $\begin{array}{l}\text { Bin } \\
\text { Range } \\
(\mu \mathrm{m})\end{array}$ & \begin{tabular}{|l|} 
Bin Mean \\
Diameter \\
$(\mu \mathrm{m})$
\end{tabular} & $\begin{array}{l}\text { Diff. of the } \\
\text { log of the } \\
\text { Size }\end{array}$ & Counts & $\begin{array}{l}\text { Weighted } \\
\text { Mean } \\
\text { Diameter }\end{array}$ \\
\hline $0.30-0.49$ & .38 & 0.21 & 30091 & \multirow{6}{*}{0.52} \\
\hline $0.50-0.99$ & .70 & 0.30 & 14155 & \\
\hline $1.00-1.99$ & 1.41 & 0.30 & 534 & \\
\hline $2.00-4.99$ & 3.16 & 0.40 & 382 & \\
\hline $5.00-9.99$ & 7.07 & 0.30 & 26 & \\
\hline$>10.00$ & $\overline{14.14}$ & 0.30 & 4 & \\
\hline
\end{tabular}

Sample \#6

\begin{tabular}{|c|c|c|c|c|}
\hline $\begin{array}{l}\text { Bin } \\
\text { Range } \\
(\mu \mathrm{m})\end{array}$ & \begin{tabular}{|l|} 
Bin Mean \\
Diameter \\
$(u m)$
\end{tabular} & $\begin{array}{l}\text { Diff. of the } \\
\log \text { of the } \\
\text { Size }\end{array}$ & Counts & $\begin{array}{l}\text { Weighted } \\
\text { Mean } \\
\text { Diamoter }\end{array}$ \\
\hline $0.30-0.49$ & .38 & 0.21 & 32393 & \multirow{6}{*}{0.52} \\
\hline $0.50-0.99$ & .70 & 0.30 & 15166 & \\
\hline $1.00-1.99$ & 1.41 & 0.30 & 556 & \\
\hline $2.00-4.99$ & 3.16 & 0.40 & 407 & \\
\hline $5.00-9.99$ & 7.07 & 0.30 & 25 & \\
\hline$>10.00$ & 14.14 & 0.30 & 22 & \\
\hline
\end{tabular}

Sample \#8

\begin{tabular}{|c|c|c|c|c|}
\hline $\begin{array}{l}\text { Bin } \\
\text { Range } \\
\text { (um) }\end{array}$ & $\begin{array}{l}\text { Bin Mean } \\
\text { Diameter } \\
\text { (um) }\end{array}$ & $\begin{array}{l}\text { Diff. of the } \\
\text { log of the } \\
\text { Size }\end{array}$ & Counts & $\begin{array}{l}\text { Weighted } \\
\text { Mean } \\
\text { Diameter }\end{array}$ \\
\hline $0.30-0.49$ & .38 & 0.21 & 31411 & \multirow{6}{*}{0.53} \\
\hline $0.50-0.99$ & .70 & 0.30 & 15016 & \\
\hline $0.00-1.99$ & 1.41 & 0.30 & 579 & \\
\hline $2.00-4.99$ & 3.16 & 0.40 & 363 & \\
\hline $5.00-9.99$ & 7.07 & 0.30 & 35 & \\
\hline$>10.00$ & 14.14 & 0.30 & 22 & \\
\hline
\end{tabular}

\begin{tabular}{|c|c|c|c|c|}
\hline $\begin{array}{l}\text { Bin } \\
\text { Range } \\
\text { (um) }\end{array}$ & \begin{tabular}{|l} 
Bin Mean \\
Diameter \\
( $\mathrm{mm})$
\end{tabular} & $\begin{array}{l}\text { Diff. of the } \\
\log \text { of the } \\
\text { Size }\end{array}$ & Counts & $\begin{array}{l}\text { Weighted } \\
\text { Mean } \\
\text { Diameter }\end{array}$ \\
\hline $0.30-0.49$ & .38 & 0.21 & 31051 & \\
\hline $0.50-0.99$ & .70 & 0.30 & 14732 & \\
\hline $1.00-1.99$ & 1.41 & 0.30 & 587 & \\
\hline $2.00-4.99$ & 3.16 & 0.40 & 411 & \\
\hline $5.00-9.99$ & 7.07 & 0.30 & 55 & \\
\hline$>10.00$ & 14.14 & 0.30 & 101 & \\
\hline
\end{tabular}




\section{Aerosol Particle Measurement Record}

Description of sample location PUREX P\&O Gallery Reuspension $.25 \mathrm{~m}$,

Date $9 / 07 / 94$, Time sample started 10:51:01, Temperature 82.1 F, Humidity $38.8 \%$, Dew Point $54.5 \mathrm{~F}$, Average Wind Velocity (if applicable) $.00 \mathrm{~m} / \mathrm{s}$, \# of physical barriers between sample area and outside (if applicable) $\underline{3}$

Sample \#1

\begin{tabular}{|c|c|c|c|c|}
\hline \begin{tabular}{|l|} 
Bin \\
Range \\
$(\mu \mathrm{m})$
\end{tabular} & \begin{tabular}{|l|} 
Bin Mean \\
Diameter \\
(um)
\end{tabular} & $\begin{array}{l}\text { Diff. of the } \\
\text { log of the } \\
\text { Size }\end{array}$ & Counts & $\begin{array}{l}\text { Weighted } \\
\text { Mean } \\
\text { Diameter }\end{array}$ \\
\hline $0.30-0.49$ & 0.38 & 0.21 & 24415 & \multirow{6}{*}{0.52} \\
\hline $0.50-0.99$ & 0.70 & 0.30 & 4658 & \\
\hline $1.00-1.99$ & 1.41 & 0.30 & 770 & \\
\hline $2.00 \cdot 4.99$ & 3.16 & 0.40 & 521 & \\
\hline $5.00-9.99$ & 7.07 & 0.30 & 39 & \\
\hline$>10.00$ & 14.14 & 0.30 & 26 & \\
\hline \multicolumn{5}{|l|}{ Sample 73} \\
\hline $\begin{array}{l}\text { Bin } \\
\text { Range } \\
(\mu \mathrm{m}) \\
\end{array}$ & \begin{tabular}{|l|} 
Bin Mean \\
Diameter \\
$(\mu \mathrm{m})$
\end{tabular} & \begin{tabular}{|l|} 
Diff. of the \\
log of the \\
Size
\end{tabular} & Counts & \begin{tabular}{|l|} 
Weighted \\
Mean \\
Diameter
\end{tabular} \\
\hline $0.30-0.49$ & 0.38 & 0.21 & 24471 & \multirow{6}{*}{0.73} \\
\hline $0.50-0.99$ & 0.70 & 0.30 & 6702 & \\
\hline $1.00-1.99$ & 1.41 & 0.30 & 2064 & \\
\hline $2.00-4.99$ & 3.16 & 0.40 & 2227 & \\
\hline $5.00-9.99$ & 7.07 & 0.30 & 135 & \\
\hline$>10.00$ & 14.14 & 0.30 & 77 & \\
\hline \multicolumn{5}{|l|}{ Sample \#5 } \\
\hline \begin{tabular}{|l} 
Bin \\
Range \\
$(\mu \mathrm{m})$ \\
\end{tabular} & \begin{tabular}{|l|}
$\begin{array}{l}\text { Bin Moan } \\
\text { Diameter } \\
(u m)\end{array}$ \\
\end{tabular} & \begin{tabular}{|l|} 
Diff. of the \\
log of the \\
Size \\
\end{tabular} & Counts & \begin{tabular}{|l} 
Weighted \\
Mean \\
Diameter
\end{tabular} \\
\hline $0.30-0.49$ & 0.38 & 0.21 & 23737 & \multirow{6}{*}{0.76} \\
\hline $0.50-0.99$ & 0.70 & 0.30 & 6045 & \\
\hline $1.00-1.99$ & 1.41 & 0.30 & 1734 & \\
\hline $2.00-4.99$ & 3.16 & 0.40 & 1933 & \\
\hline $5.00-9.99$ & 7.07 & 0.30 & 164 & \\
\hline$>10.00$ & 14.14 & 0.30 & 195 & \\
\hline
\end{tabular}

\begin{tabular}{|c|c|c|c|c|}
\hline \multicolumn{5}{|l|}{ Sample \#7 } \\
\hline $\begin{array}{l}\text { Bin } \\
\text { Range } \\
(\mu \mathrm{m})\end{array}$ & \begin{tabular}{|l|} 
Bin Mean \\
Diameter \\
$(\mu \mathrm{m})$
\end{tabular} & $\begin{array}{l}\text { Diff. of the } \\
\log \text { of the } \\
\text { Size }\end{array}$ & Counts & $\begin{array}{l}\text { Weighted } \\
\text { Mean } \\
\text { Diamoter }\end{array}$ \\
\hline $0.30-0.49$ & 0.38 & 0.21 & 25049 & \multirow{6}{*}{0.49} \\
\hline $0.50-0.99$ & 0.70 & 0.30 & 4495 & \\
\hline $1.00-1.99$ & $\overline{1.41}$ & 0.30 & 624 & \\
\hline $2.00-4.99$ & 3.16 & 0.40 & 352 & \\
\hline $5.00-9.99$ & 7.07 & 0.30 & 10 & \\
\hline$>10.00$ & 14.14 & 0.30 . & 6 & \\
\hline \multicolumn{5}{|l|}{ Sample \#9 } \\
\hline \begin{tabular}{|l} 
Bin \\
Range \\
$(u \mathrm{~m})$
\end{tabular} & \begin{tabular}{|l} 
Bin Mean \\
Diameter \\
$(u m)$
\end{tabular} & $\begin{array}{l}\text { Diff. of the } \\
\text { log of the } \\
\text { Size }\end{array}$ & Counts & $\begin{array}{l}\text { Weighted } \\
\text { Mean } \\
\text { Diameter }\end{array}$ \\
\hline $0.30-0.49$ & 0.38 & 0.21 & 25681 & \multirow{6}{*}{0.57} \\
\hline $0.50-0.99$ & 0.70 & 0.30 & 5062 & \\
\hline $1.00-1.99$ & 1.41 & 0.30 & 971 & \\
\hline $2.00-4.99$ & 3.16 & 0.40 & 815 & \\
\hline $5.00-9.99$ & 7.07 & 0.30 & 67 & \\
\hline$>10.00$ & 14.14 & 0.30 & 61 & \\
\hline
\end{tabular}

Sample \#2

\begin{tabular}{|c|c|c|c|c|}
\hline $\begin{array}{l}\text { Bin } \\
\text { Range } \\
(u m)\end{array}$ & \begin{tabular}{|l} 
Bin Mean \\
Diameter \\
$(u m)$
\end{tabular} & $\begin{array}{l}\text { Diff. of the } \\
\text { log of the } \\
\text { Size }\end{array}$ & Counts & $\begin{array}{l}\text { Weighted } \\
\text { Mean } \\
\text { Diamoter }\end{array}$ \\
\hline $0.30-0.49$ & .38 & 0.21 & 13467 & \\
\hline $0.50-0.99$ & .70 & 0.30 & 23065 & \\
\hline $1.00-1.99$ & 1.41 & 0.30 & 25175 & \\
\hline $2.00-4.99$ & 3.16 & 0.40 & 89556 & \\
\hline $5.00-9.99$ & 7.07 & $\overline{0.30}$ & 29186 & \\
\hline$>10.00$ & 14.14 & 0.30 & 32708 & \\
\hline
\end{tabular}

\begin{tabular}{|c|c|c|c|c|}
\hline \multicolumn{5}{|l|}{ Sample \#4 } \\
\hline $\begin{array}{l}\text { Bin } \\
\text { Range } \\
(\mathrm{um})\end{array}$ & \begin{tabular}{|l|} 
Bin Mean \\
Diameter \\
$(\mathrm{um})$
\end{tabular} & $\begin{array}{l}\text { Diff. of the } \\
\text { log of the } \\
\text { Size }\end{array}$ & Counts & $\begin{array}{l}\text { Weighted } \\
\text { Mean } \\
\text { Diameter }\end{array}$ \\
\hline $0.30-0.49$ & .38 & 0.21 & 24927 & \multirow{6}{*}{0.51} \\
\hline $0.50-0.99$ & .70 & 0.30 & 4502 & \\
\hline $1.00-1.99$ & 1.41 & 0.30 & 685 & \\
\hline $2.00-4.99$ & 3.16 & 0.40 & 426 & \\
\hline $5.00-9.99$ & 7.07 & 0.30 & 44 & \\
\hline$>10.00$ & 14.14 & 0.30 & 26 & \\
\hline
\end{tabular}

\section{Sample \#6}

\begin{tabular}{|c|c|c|c|c|}
\hline $\begin{array}{l}\text { Bin } \\
\text { Range } \\
(\mu \mathrm{m})\end{array}$ & $\begin{array}{l}\text { Bin Mean } \\
\text { Diameter } \\
(\mu \mathrm{m})\end{array}$ & $\begin{array}{l}\text { Diff. of the } \\
\log \text { of the } \\
\text { Size }\end{array}$ & Counts & \begin{tabular}{|l|} 
Weighted \\
Mean \\
Diameter
\end{tabular} \\
\hline $0.30-0.49$ & .38 & 0.21 & 24577 & \multirow{6}{*}{0.61} \\
\hline $0.50-0.99$ & .70 & 0.30 & $\overline{5127}$ & \\
\hline $1.00-1.99$ & 1.41 & 0.30 & 1052 & \\
\hline $2.00-4.99$ & 3.16 & 0.40 & 1022 & \\
\hline $5.00-9.99$ & 7.07 & 0.30 & $\overline{74}$ & \\
\hline$>10.00$ & 14.14 & 0.30 & 97 & \\
\hline
\end{tabular}

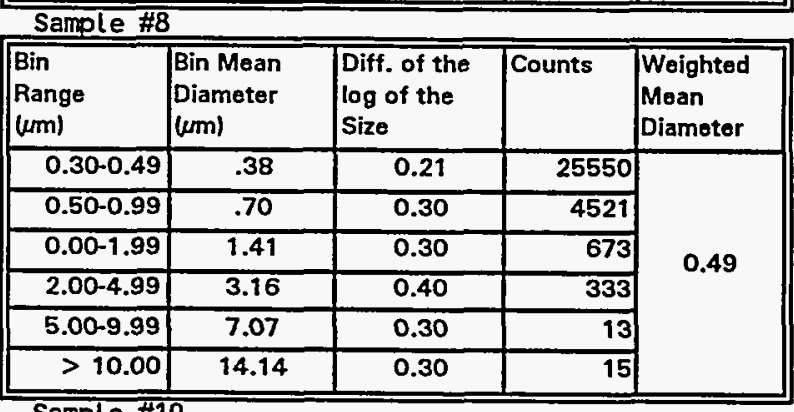

\begin{tabular}{|l|l|l|r|r||}
\hline Sample \#10 & \multicolumn{3}{|l||}{} \\
\hline $\begin{array}{l}\text { Bin } \\
\text { Range } \\
(\mathrm{mm})\end{array}$ & $\begin{array}{l}\text { Bin Mean } \\
\text { Diameter } \\
(\mathrm{mm})\end{array}$ & $\begin{array}{l}\text { Diff. of the } \\
\text { log of the } \\
\text { Size }\end{array}$ & Counts & $\begin{array}{l}\text { Weighted } \\
\text { Mean } \\
\text { Diameter }\end{array}$ \\
\hline $0.30-0.49$ & .38 & 0.21 & 26074 & \\
\hline $0.50-0.99$ & .70 & 0.30 & 4539 & \\
\hline $1.00-1.99$ & 1.41 & 0.30 & 641 & 0.49 \\
\hline $2.00-4.99$ & 3.16 & 0.40 & 383 & \\
\hline $5.00-9.99$ & 7.07 & 0.30 & 18 & \\
\hline$>10.00$ & 14.14 & 0.30 & 12 & \\
\hline
\end{tabular}




\section{DISTRIBUTION}

\section{Number of Copies}

\section{OFFSITE}

3

University of Washington

Department of Industrial Hygiene and Safety

Seattle, Washington 98195

M. S. Morgan, SCD.

ONSITE

1 U.S. Department of Energy,

Richland Operations office

1 DOE-RL PubTic Reading Room Al-65

Westinghouse Hanford Company

5

D. B. Ottley

T7-37

1

R. M. Rogers

R3-12

1

T. D. Merkling

T7-37

1

Central Files

L8-04

1

Document Processing and Distribution

L8-15

2 OSTI

$+87$

EG-4Y 